\title{
EXPERIMENTAL AND COMPUTATIONAL METHODS FOR INVESTIGATING AUTOMOTIVE DOOR CLOSURE SOUNDS
}

By

Giuseppe Teodorico Valentino Garro

A thesis submitted to the Department of Mechanical and Materials Engineering

In conformity with the requirements for the degree of

Master of Applied Science

\author{
Queen's University \\ Kingston, Ontario, Canada
}

(July, 2018)

Copyright @Giuseppe Teodorico Valentino Garro, 2018 


\begin{abstract}
The focus of this investigation was to examine the acoustic trends present during operation of an automotive door closure at two impact speeds using experimental and computational methods. The impact speeds were $0.851 \mathrm{~m} / \mathrm{s}$ and $1.179 \mathrm{~m} / \mathrm{s}$. Transient sound pressure measurements from five different door closure mechanisms were collected in a semi-anechoic chamber using a threeelement condenser microphone array. Post-processing methodologies such as Sound Pressure Level versus 1/3 Octave Bandwidth and Continuous Wavelet Transform computations were conducted. These procedures provided an in-depth analysis on the overall generated sound in addition to identifying which frequencies dominate the response at the same time as specific impact events during latch operation. Sound quality metrics such as loudness and sharpness were used to explore how the impulsive sound relates to a consumer's impression of the sound. It was suggested in past research that individuals prefer sounds that possess a 'deep' sound characteristic as opposed to sounds characterized as 'metallic'. It was revealed that the overall sound quality was mainly influenced by the frequency composition of the sound.
\end{abstract}

The focus of this study was the sound generated by the impact events present during the latchlocking operation. With ANSYS Rigid Body Dynamics and Explicit Dynamics, it was concluded that two impact events within the latch influenced the acoustic response. The impacts occurred between the Striker/Over-Slam Bumper \#1 and the Ratchet/Housing. These were labelled as the primary and secondary impact events respectively. Investigation of the experimental sound data revealed that the faster entrance speed $(1.179 \mathrm{~m} / \mathrm{s})$ produced a sound with a larger normalized magnitude. Furthermore, the additional energy allowed frequencies approximately $2.5 \mathrm{kHz}$ and 
below to become more pronounced. The findings suggest that increasing the closing speed could produce a "more preferred" sound based on psychoacoustic principles.

A computational acoustic analysis using ANSYS Workbench was performed to complement the experimental analysis. Only the primary impact event was simulated due to the inherent limitations of the workstation used to perform the analysis. Similarities between the computational and experimental data were present. However, it is recommended that an alternate simulation software that is capable of modelling "physical impacts" be used. 


\section{Co-Authorship}

This work is solely written by the author, Giuseppe Teodorico Valentino Garro. 


\section{Acknowledgements}

I take this opportunity to thank my supervisor Dr. Chris Mechefske for his guidance and encouragement. Dr. Mechefske was steadfast in his support, and he helped guide my path to success, especially when faced with challenges.

I wish to extend my gratitude to Orest Kostiv and Krystof Jankowski at Magna Closures Inc. for their technical support and guidance.

I express my thanks to the Natural Science and Engineering Research Council of Canada (NSERC) for financial support of this work.

I would also like to thank my professional colleagues of the System Dynamics Research Group (SDRG) and the Department of Mechanical and Materials Engineering at Queen's University for continuous support on this journey.

I extend my sincere thanks to my family and friends who have always been there for me throughout my academic career. I would not have become the person I am today without their constant encouragement.

Most importantly, I would like to thank my dearest Elena for her unwavering support, humour and encouragement since the beginning of my graduate career. 


\section{Table of Contents}

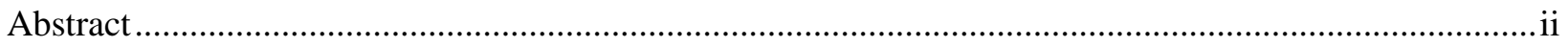

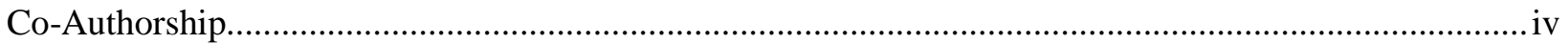

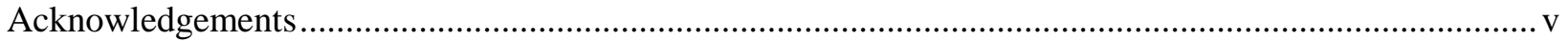

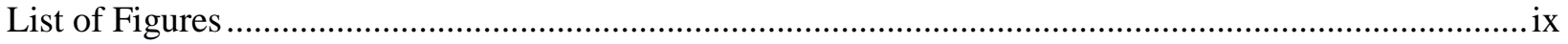

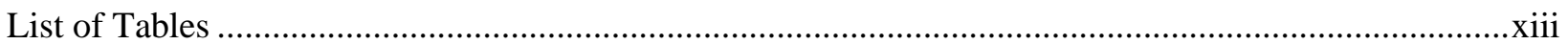

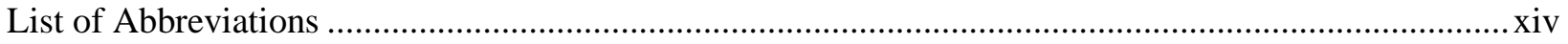

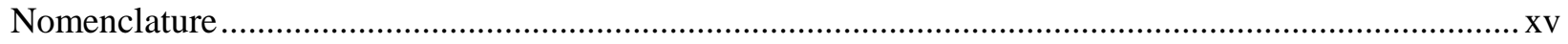

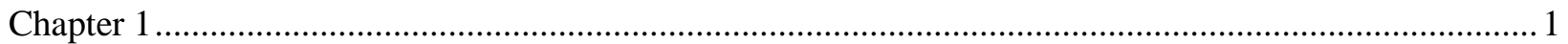

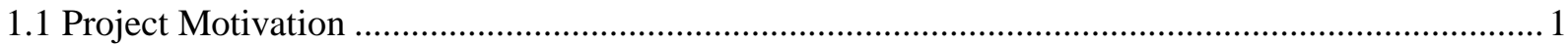

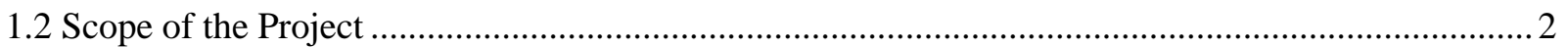

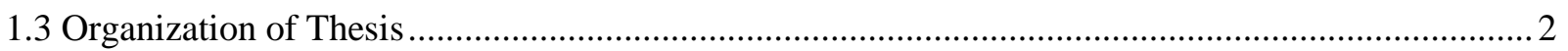

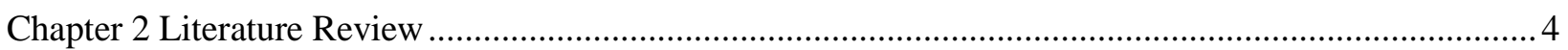

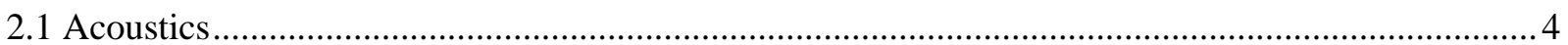

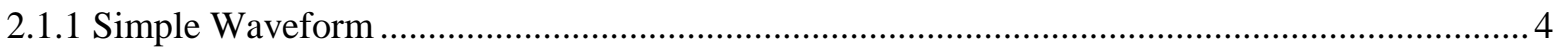

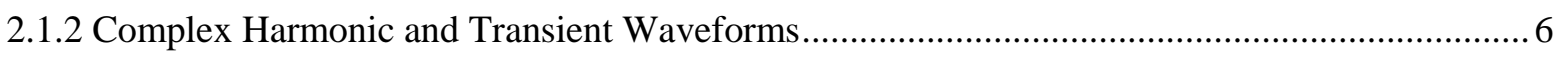

2.2 Vehicle Door Closure System Acoustics .................................................................................... 8

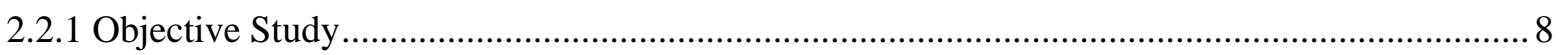

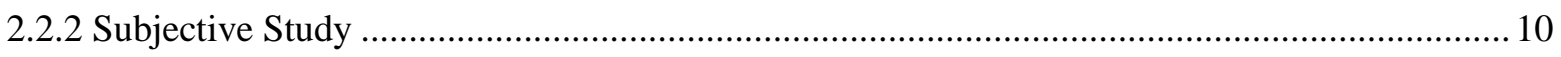

2.3 D21MC Automotive Door Closure System ........................................................................... 12

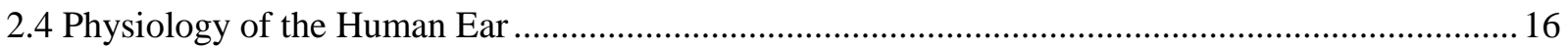

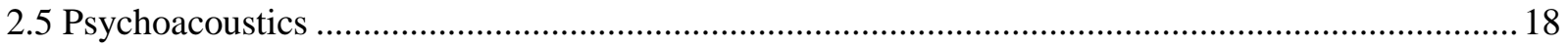

2.5.1 Sound Quality Metrics: Sharpness and Loudness ...............................................................20

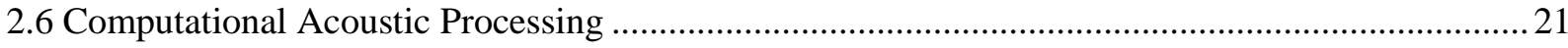

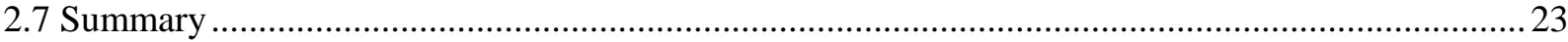

Chapter 3 Experimental Procedures and Data Analysis .....................................................................25

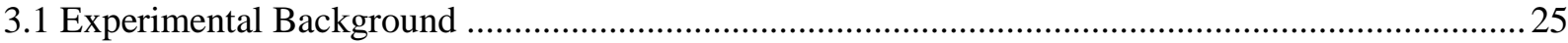

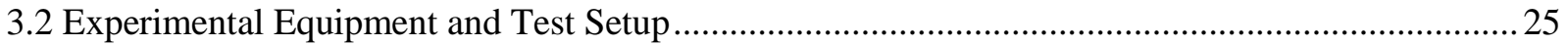

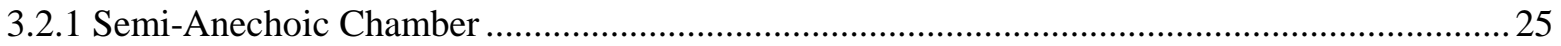

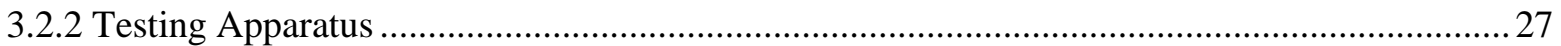

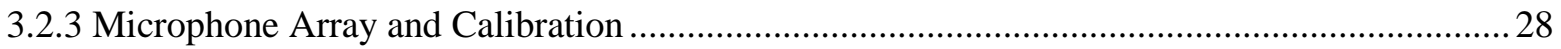

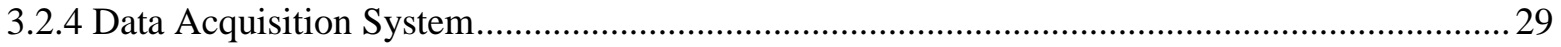

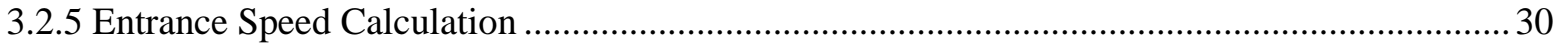




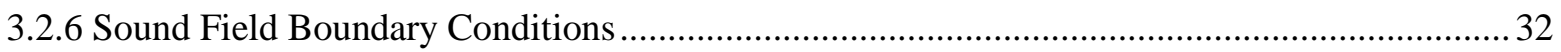

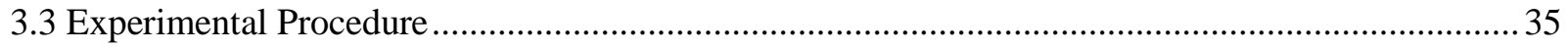

3.4 Experimental Post-Processing Procedure and Methodologies........................................................36

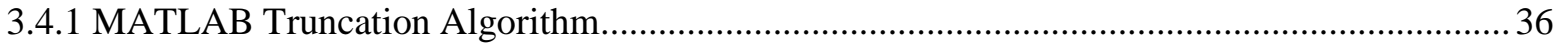

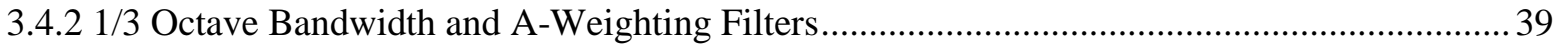

3.4.3 Continuous Wavelet Transformation Analysis ...................................................................... 44

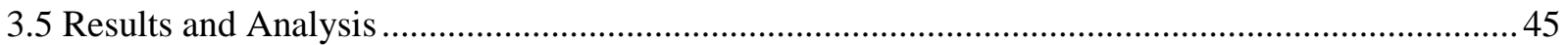

3.5.1 Continuous Wavelet Transform Analysis ............................................................................... 48

3.5.2 Sound Pressure Level versus 1/3 Octave Band ...................................................................52

3.5.3 Sound Quality Metrics: Loudness and Sharpness .................................................................58

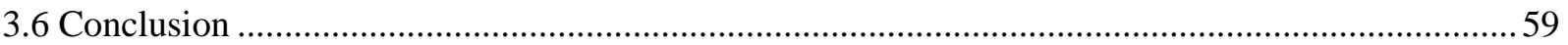

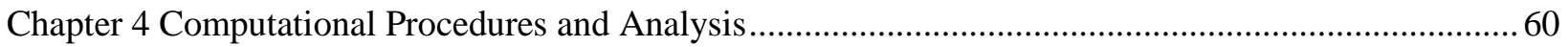

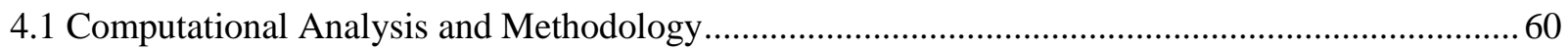

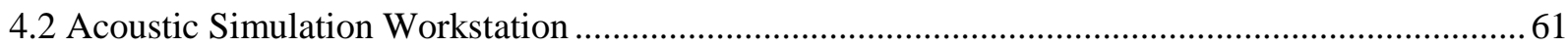

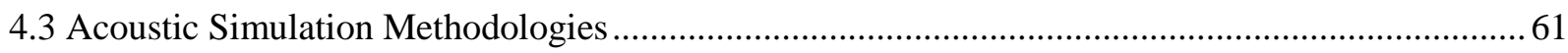

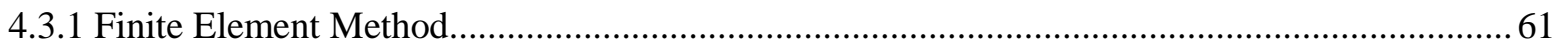

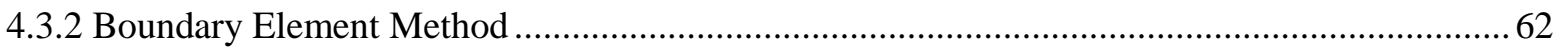

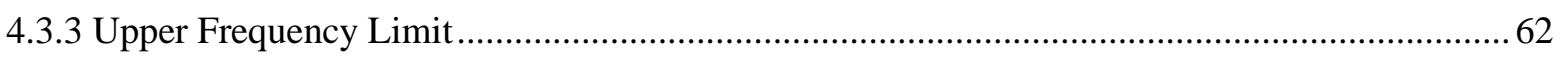

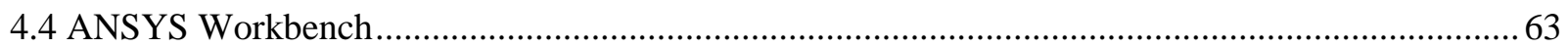

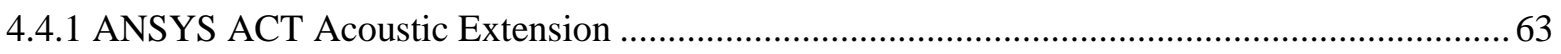

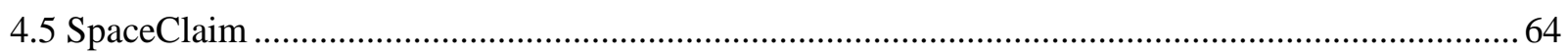

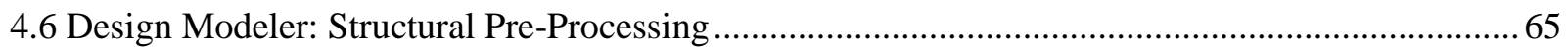

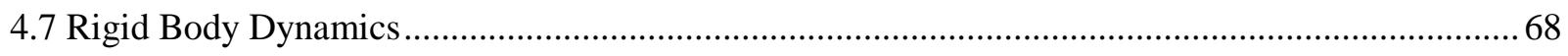

4.7.1 Coil Spring to Torsional Spring Conversion.................................................................. 68

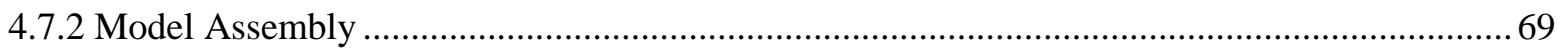

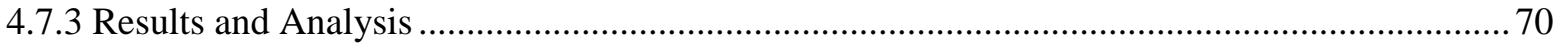

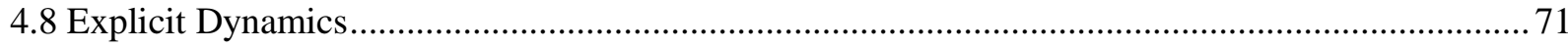

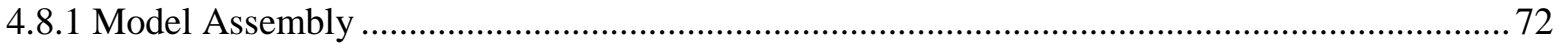

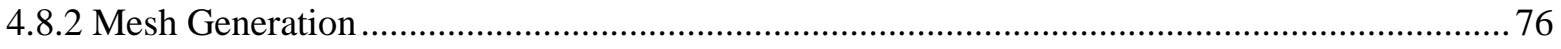

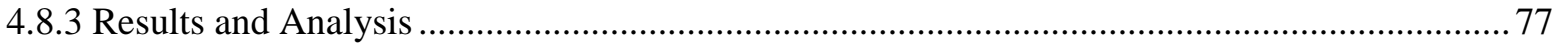

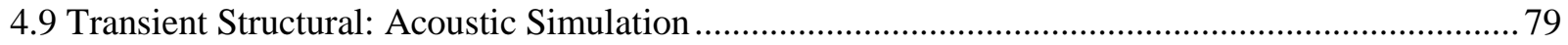

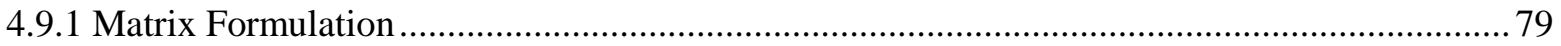

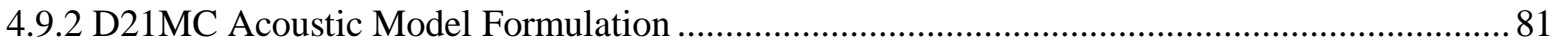

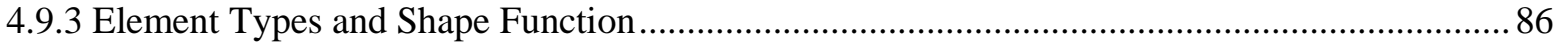




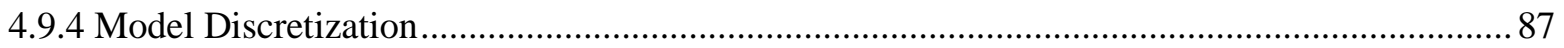

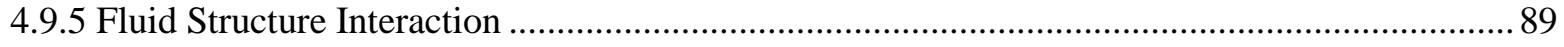

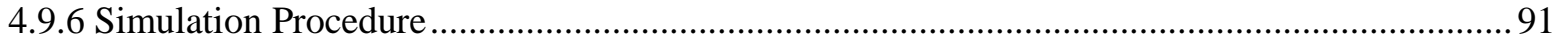

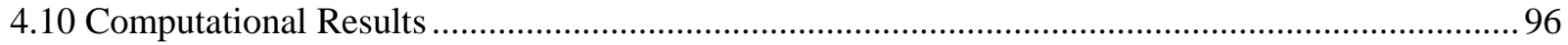

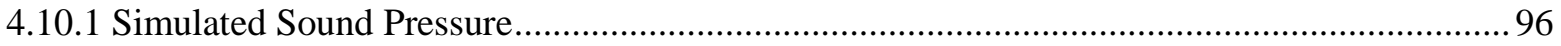

4.10.2 Continuous Wavelet Transform of the Simulated Data.................................................... 100

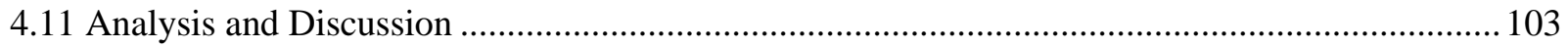

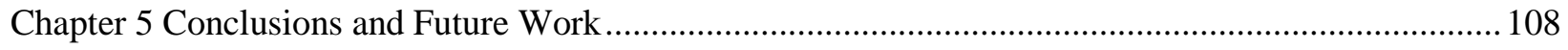

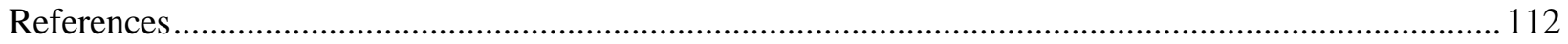

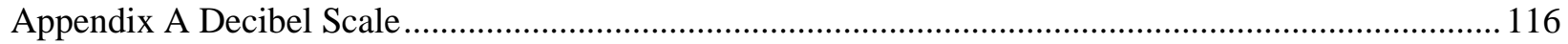

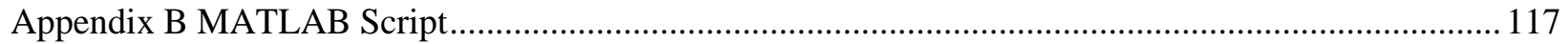

Appendix C MATLAB Script: Incoherent Sound Pressure Level Addition.......................................... 131

Appendix D Transient Sound Pressure and CWT Plots.......................................................................... 135

Appendix E Tabular Average Sound Pressure Level.............................................................................. 140

Appendix F Rigid Body Dynamics: Coil Spring to Torsional Spring Conversion ................................. 143

Appendix G Computational Analysis Flow-Chart ............................................................................ 146 


\section{List of Figures}

Figure 2.1: a) Longitudinal waveform. b) Transverse waveform. …...................................................5

Figure 2.2: Complex waveforms and corresponding spectrogram of various musical instruments............ 7

Figure 2.3: Transient acoustic waveform of a door closure sound used in the objective study.................. 9

Figure 2.4: CWT of the recorded door sounds of Subject A and Subject B respectfully. The colour bar

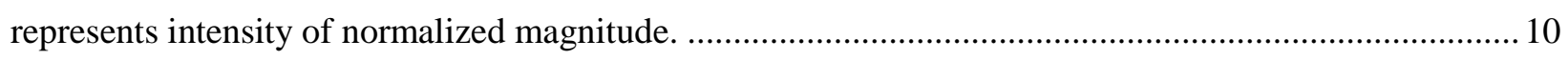

Figure 2.5: Subjective results of one stimuli based on acoustic descriptors. Diamond and circle points

refer to the German and Japanese participants respectively. .................................................................. 11

Figure 2.6: D21MC Latch Assembly A) Isometric view, B) Side view, C) Front view............................ 13

Figure 2.7: A) Pawl and pawl encapsulation. B) Ratchet and ratchet encapsulation. C) Striker ................ 14

Figure 2.8: Latch locking progression. A) Fully un-latched position. B) Secondary latched position. C)

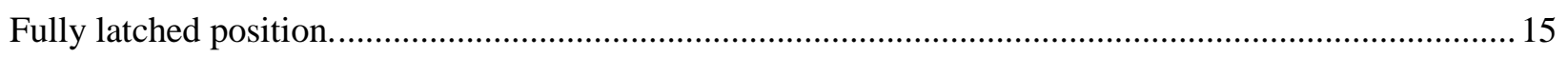

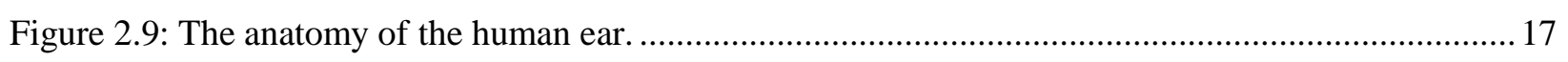

Figure 2.10: Subjective evaluation between four vehicle door stimuli..................................................20

Figure 2.11: Comparison of the experimental and computational results of the washing machine study.. 23

Figure 3.1: Simplified layout of the semi-anechoic chamber with length $(\mathrm{L})=7.62 \mathrm{~m}$, width $(\mathrm{w})=5.41 \mathrm{~m}$, and height $(\mathrm{h})=2.8 \mathrm{~m}$. The red box indicates the location of the apparatus during testing. .....................26

Figure 3.2: Semi-anechoic chamber lined with sound absorbing material. ............................................26

Figure 3.3: The latch mechanism is highlighted by the red circle and the striker is highlighted by the blue

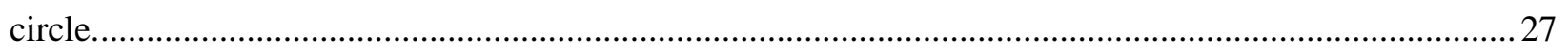

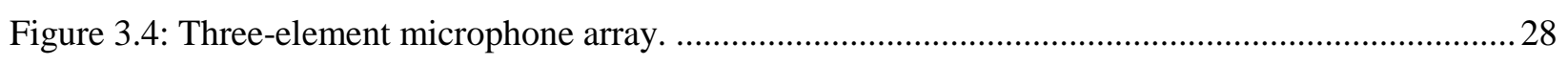

Figure 3.5: Side (left) and front (right) view of the microphones normal to the latch............................29

Figure 3.6: Speed trap device attached to the pendulum apparatus. ..................................................... 31

Figure 3.7: Metal rods used to achieve $1.179 \mathrm{~m} / \mathrm{s}$ (top) and $0.851 \mathrm{~m} / \mathrm{s}$ (bottom) entrance speeds. .............. 31

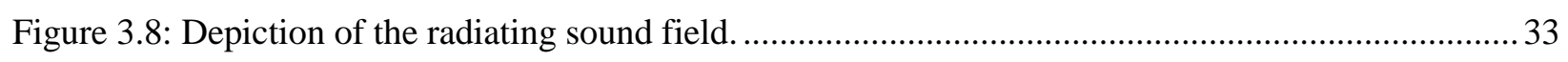

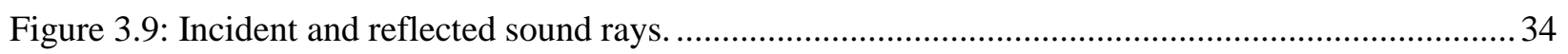

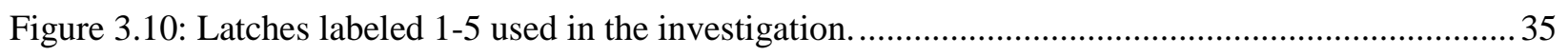

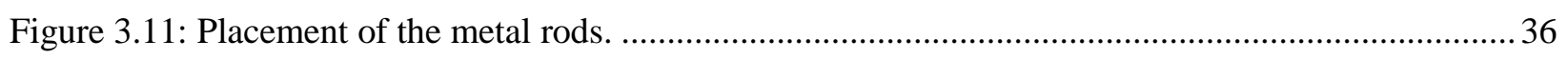

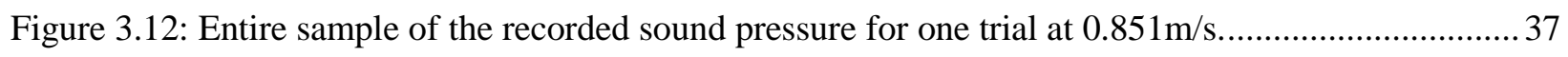

Figure 3.13: Extracted latch operation sound pressure waveform for one trial at $0.851 \mathrm{~m} / \mathrm{s} \ldots \ldots \ldots \ldots \ldots \ldots . . . . . .38$

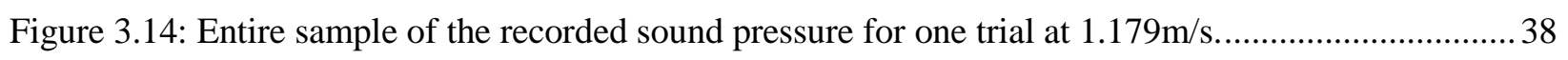

Figure 3.15: Extracted latch operation sound pressure waveform for one trial at $1.179 \mathrm{~m} / \mathrm{s} \ldots \ldots \ldots \ldots \ldots \ldots \ldots . . . . . . .39$ 
Figure 3.16: Revised Equal Loudness Contour plot stated in ISO 226 [35]. The binaural threshold is denoted as the lowest level of sound a human can hear.

Figure 3.17: "A", "B" and "C" weighting networks. Gain $[\mathrm{dB}]$ represented by the y-axis and frequency

$[\mathrm{Hz}]$ on the $\mathrm{x}$-axis 41

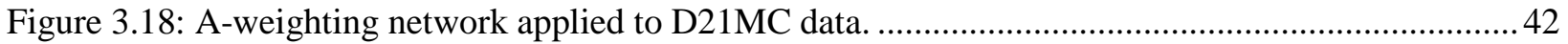

Figure 3.19: Class $11 / 3$ octave filter with center frequency $1 \mathrm{kHz}$ applied to the $\mathrm{D} 21 \mathrm{MC}$ data. ..............43

Figure 3.20: SPL versus 1/3 octave bandwidth for Latch 3 at $0.851 \mathrm{~m} / \mathrm{s}$ entrance speed......................... 44

Figure 3.21: Segmented waveform of the $0.851 \mathrm{~m} / \mathrm{s}$ closure speed........................................................46

Figure 3.22: Segmented waveform of the $1.179 \mathrm{~m} / \mathrm{s}$ closure speed.......................................................46

Figure 3.23: CWT of the $0.851 \mathrm{~m} / \mathrm{s}$ closure speed measured at MP1 ....................................................48

Figure 3.24: CWT of the $0.851 \mathrm{~m} / \mathrm{s}$ closure speed measured at MP2 .................................................... 49

Figure 3.25: CWT of the $0.851 \mathrm{~m} / \mathrm{s}$ closure speed measured at MP3 .................................................... 49

Figure 3.26: CWT of the $1.179 \mathrm{~m} / \mathrm{s}$ closure speed measures at MP1 ....................................................50

Figure 3.27: CWT of the $1.179 \mathrm{~m} / \mathrm{s}$ closure speed measures at MP2 ..................................................51

Figure 3.28: CWT of the $1.179 \mathrm{~m} / \mathrm{s}$ closure speed measures at MP3 .....................................................51

Figure 3.29: A-weighted average SPL versus $1 / 3$ octave band of Latch 1 . ................................................53

Figure 3.30: A-weighted average SPL versus $1 / 3$ octave band of Latch 2 .............................................53

Figure 3.31: A-weighted average SPL versus 1/3 octave band of Latch 3 ............................................54

Figure 3.32: A-weighted average SPL versus $1 / 3$ octave band of Latch 4 . ............................................54

Figure 3.33: A-weighted average SPL versus $1 / 3$ octave band of Latch 5 ............................................55

Figure 3.34: A-weighted average SPL versus $1 / 3$ octave band of all latches. ..........................................56

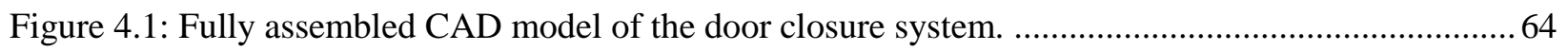

Figure 4.2: Fully assembled and repositioned door closure system..................................................... 65

Figure 4.3: Original pawl insert (Top). Simplified, multi-body pawl insert (Bottom). ............................ 66

Figure 4.4: Original ratchet insert (Left). Simplified, multi-body ratchet insert (Right).........................66

Figure 4.5: Original striker (Left). Simplified, multi-body striker (Right)...........................................67

Figure 4.6: Pawl and ratchet coil spring travel path during latch operation. ...........................................69

Figure 4.7: Tracked speed of the pawl encapsulation motion via a velocity probe. ................................. 70

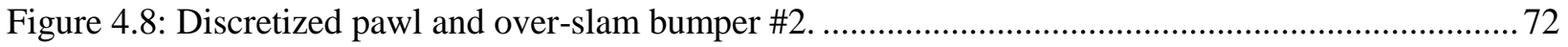

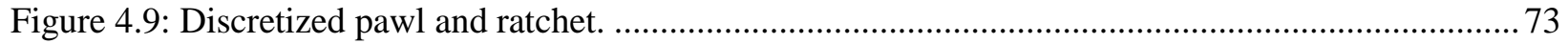

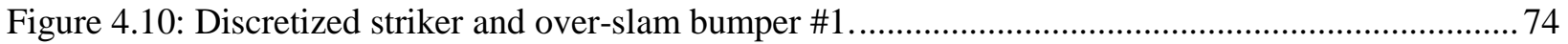

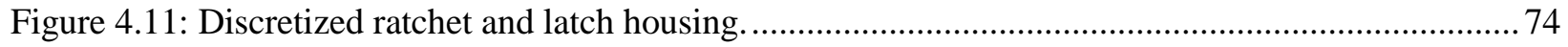

Figure 4.12: Reaction force results of the Striker/Over-Slam Bumper \#1 impact event at $0.851 \mathrm{~m} / \mathrm{s} . . . . . . . .77$

Figure 4.13: Reaction force results of the Striker/Over-Slam Bumper \#1 impact event at $1.179 \mathrm{~m} / \mathrm{s} . . . \ldots . . .78$ 
Figure 4.14: Placement of fluid elements for modelling a free field environment. 82

Figure 4.15: Inadequate acoustic regions resulting from the inherent limitations of the workstation........ 83

Figure 4.16: Slivered acoustic region geometry used for TS simulations. .84

Figure 4.17: Meshed (Bottom) and not meshed (Top) MP3 acoustic region. Colours were used to identify bodies for mesh generation. Virtual microphone located $0.5 \mathrm{~m}$ from the latch center represented by the red marker. The latch center is represented by the green marker and is common for all acoustic simulations.

Figure 4.18: Meshed (Bottom) and not meshed (Top) MP2 acoustic region. Virtual microphone located $0.5 \mathrm{~m}$ from the latch center represented by the red marker. .85

Figure 4.19: Meshed (Bottom) and not meshed (Top) MP1 acoustic region. Virtual microphone located $0.5 \mathrm{~m}$ from the latch center represented by the red marker.

Figure 4.20: depiction of the gradual mesh density transition from the latch center towards the outer

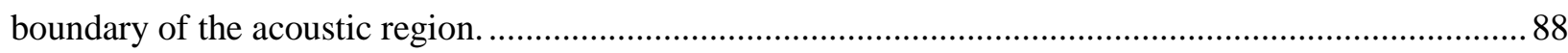

Figure 4.21: Fluid structure interaction between structural and acoustic elements. . .90

Figure 4.22: Discretized latch components (grey and white) and acoustic region (yellow) with FSI enabled. 91

Figure 4.23: Latch body defined as Contact surface shown in red and the acoustic Target surface shown in yellow...... . .92

Figure 4.24: Fixed boundary condition shown in blue. .92

Figure 4.25: Forces applied at nodes for the bumper (Top) and striker (Bottom). .93

Figure 4.26: Definition of the acoustic absorption surface .95

Figure 4.27: Sound pressure measured at MP3 0.5m from the latch. .96

Figure 4.28: Additional microphones added within the acoustic region. 97

Figure 4.29: Sound pressure result at MP3_Mid. …............................................................................. 98

Figure 4.30: Sound pressure results at MP3_Close. .............................................................................. 98

Figure 4.31: Sound pressure result at MP1_Close............................................................................... 99

Figure 4.32: Sound pressure result at MP2_Close............................................................................... 99

Figure 4.33: Continuous wavelet analysis of the $0.851 \mathrm{~m} / \mathrm{s}$ TS analysis at MP1_Close......................... 100

Figure 4.34: Continuous wavelet analysis of the $0.851 \mathrm{~m} / \mathrm{s}$ TS analysis at MP2_Close. ......................... 100

Figure 4.35: Continuous wavelet analysis of the $0.851 \mathrm{~m} / \mathrm{s}$ TS analysis at MP3_Close. ....................... 101

Figure 4.36: Continuous wavelet analysis of the 1.179m/s TS analysis at MP1_Close......................... 101

Figure 4.37: Continuous wavelet analysis of the $1.179 \mathrm{~m} / \mathrm{s}$ TS analysis at MP2_Close........................ 102

Figure 4.38: Continuous wavelet analysis of the $1.179 \mathrm{~m} / \mathrm{s}$ TS analysis at MP3_Close. ......................... 102 
Figure 4.39: Comparison of simulated (A) and experimental (B) primary impact sound pressure of the

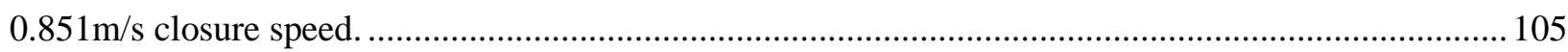




\section{List of Tables}

Table 2.1: Material properties of the latch components used in door locking operation (provided by

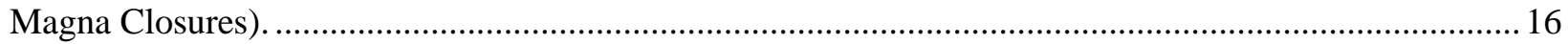

Table 3.1: Calibration settings for the microphone array. …..................................................................29

Table 3.2: Approximate CWT Dominant Frequencies of the primary and secondary impacts.................50

Table 3.3: Tabular average SPL results of the $0.851 \mathrm{~m} / \mathrm{s}$ closure speed.................................................56

Table 3.4: Tabular average SPL results of the $1.179 \mathrm{~m} / \mathrm{s}$ closure speed.................................................57

Table 3.5: Percent difference in average SPL between the $0.851 \mathrm{~m} / \mathrm{s}$ and $1.179 \mathrm{~m} / \mathrm{s}$ closure speeds. .........57

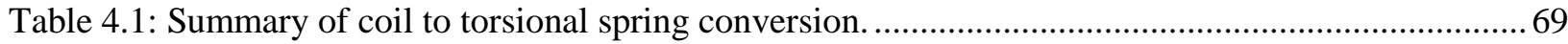

Table 4.2: Tangential impact speeds of the latch components.............................................................. 71

Table 4.3: Mesh metric and statistic results of the explicit dynamic analyses....................................... 76

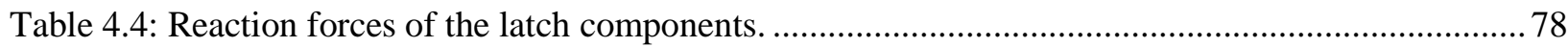

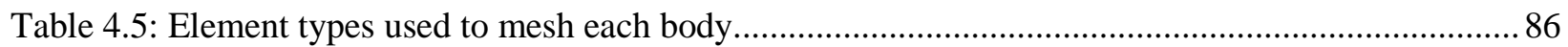

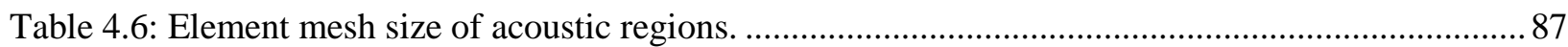

Table 4.7: Mesh metric and statistic results of the transient structural acoustic analyses. ........................89

Table 4.8: Stepwise application of the force generated during the $0.851 \mathrm{~m} / \mathrm{s}$ entrance speed...................94

Table 4.9: Stepwise application of the force generated during the $1.179 \mathrm{~m} / \mathrm{s}$ entrance speed...................94

Table 4.10: Material properties of the acoustic fluid domain. .................................................................... 94 


\section{List of Abbreviations}

The following abbreviations and acronyms are found within this thesis. They are defined here.

\begin{tabular}{|c|c|}
\hline ASA & Acoustical Society of America \\
\hline ACT & Application Customization Toolkit \\
\hline BEM & Boundary Element Method \\
\hline $\mathrm{COC}$ & Coefficient of Correlation \\
\hline CAD & Computer Aided Design \\
\hline CWT & Continuous Wavelet Transform \\
\hline DAQ & Data Acquisition System \\
\hline DoF & Degree of Freedom \\
\hline DM & Design Modeler \\
\hline EPW & Elements Per Wavelength \\
\hline ELC & Equal Loudness Contour \\
\hline $\mathrm{ED}$ & Explicit Dynamics \\
\hline FEA & Finite Element Analysis \\
\hline FEM & Finite Element Method \\
\hline FL & Free Length \\
\hline FLP & Fully Latched Position \\
\hline GNF & Geometric Near Field \\
\hline $\mathrm{HNF}$ & Hydrodynamic Near Field \\
\hline $\mathrm{IL}$ & Installed Length \\
\hline IEC & International Electrotechnical Commission \\
\hline $\mathrm{MC}$ & Max Compression \\
\hline MP1 & Microphone Position 1 \\
\hline MP2 & Microphone Position 2 \\
\hline MP3 & Microphone Position 3 \\
\hline $\mathrm{NVH}$ & Noise, Vibration, and Harshness \\
\hline RBD & Ridged Body Dynamics \\
\hline STFT & Short-Time Fourier Transform \\
\hline SPL & Sound Pressure Level \\
\hline $\mathrm{SC}$ & SpaceClaim \\
\hline SEA & Statistical Energy Analysis \\
\hline TS & Transient Structural \\
\hline
\end{tabular}




\section{Nomenclature}

\begin{tabular}{|c|c|}
\hline $\mathrm{Hz}$ & Hertz \\
\hline$\lambda$ & Wavelength \\
\hline$f$ & Frequency \\
\hline s & Second \\
\hline $\mathrm{t}$ & Time \\
\hline$\rho$ & Density \\
\hline$E$ & Elastic Modulus \\
\hline $\mathrm{m}$ & Meter \\
\hline $\mathrm{L}$ & Length \\
\hline $\mathrm{w}$ & Width \\
\hline $\mathrm{h}$ & Height \\
\hline $\mathrm{V}$ & Volt \\
\hline $\mathrm{Pa}$ & Pascal \\
\hline$\nabla^{2}$ & Laplacian Operator \\
\hline $\mathrm{p}$ & pressure \\
\hline $\mathrm{c}$ & Speed of Sound \\
\hline$f_{N}$ & Nyquist Frequency \\
\hline$V_{\text {rel }}$ & Relative Speed \\
\hline$w_{s t}$ & Width of Moving Object \\
\hline$t_{s t}$ & Duration of Passing Object \\
\hline$\gamma$ & Far Field Parameter \#1 \\
\hline $\mathrm{r}$ & Distance/Radius \\
\hline$l$ & Characteristic Source Dimension \\
\hline$\kappa$ & Far Field Parameter \#2 \\
\hline $\mathrm{dB}$ & Decibel \\
\hline dBA & A-Weighted Decibel \\
\hline$s$ & Window Scaling Parameter \\
\hline$\tau$ & Window Shifting Parameter \\
\hline$((\tau-t) / s)$ & "Mother" Wavelet \\
\hline $\mathrm{N}$ & Newton \\
\hline $\mathrm{rad}$ & Radian \\
\hline$c$ & Spring Element Damping \\
\hline[]$^{\circ}$ & Degrees \\
\hline
\end{tabular}




\begin{tabular}{|c|c|}
\hline$N_{i}$ & Set of Shape Functions \\
\hline$p_{i}$ & Acoustic Nodal Pressure \\
\hline$\left[M_{f}\right]$ & Fluid Mass Matrix \\
\hline$\left[K_{f}\right]$ & Fluid Stiffness Matrix \\
\hline$\left[C_{f}\right]$ & Fluid Damping Matrix \\
\hline$\left\{F_{f}\right\}$ & Fluid Force Vector \\
\hline$\{p\}$ & Vector of Acoustic Pressure \\
\hline$\{\dot{p}\}$ & Vector of First Derivative of Acoustic Pressure \\
\hline$\{\ddot{p}\}$ & $\begin{array}{l}\text { Vector of Second Derivative of Acoustic } \\
\text { Pressure }\end{array}$ \\
\hline$\left[M_{s}\right]$ & Structural Mass Matrix \\
\hline$\left[K_{s}\right]$ & Structural Stiffness Matrix \\
\hline$\left[C_{s}\right]$ & Structural Damping Matrix \\
\hline$\left\{F_{s}\right\}$ & Structural Force Vector \\
\hline$\{u\}$ & Vector of Structural Displacement \\
\hline$\{\dot{u}\}$ & $\begin{array}{l}\text { Vector of First Derivative of Structural } \\
\text { Displacement }\end{array}$ \\
\hline$\{\ddot{u}\}$ & $\begin{array}{l}\text { Vector of Second Derivative of Structural } \\
\text { Displacement }\end{array}$ \\
\hline$\rho_{0}$ & Acoustic Fluid Density \\
\hline$[R]$ & Coupling Matrix \\
\hline[]$^{T}$ & Transpose of Matrix \\
\hline$\lambda_{\max }$ & Maximum Wavelength \\
\hline$\lambda_{\min }$ & Minimum Wavelength \\
\hline$f_{\max }$ & Maximum Frequency \\
\hline$f_{\min }$ & Minimum Frequency \\
\hline$R_{\text {acoustic }}$ & Radius of Acoustic Region \\
\hline$S_{E}$ & Element Size \\
\hline $\mathrm{kg}$ & Kilogram \\
\hline$\alpha$ & Attenuation Coefficient \\
\hline$I_{\text {absorbed }}$ & Absorbed Sound Power \\
\hline Incident & Incident Sound Power \\
\hline$C_{a}$ & Speed of Stress/Shock Wave \\
\hline$E_{\text {min }}$ & Smallest Element Dimension \\
\hline$S_{f}$ & Scale Factor \\
\hline$\Delta t$ & Stable Time Step \\
\hline
\end{tabular}




$\begin{array}{cl}V o l & \text { Volume of Contact Segment } \\ \mu & \text { Bulk Modulus of Contact Element } \\ A & \text { Area of Contact Segment } \\ \beta & \text { Penalty Factor } \\ \delta & \text { Degree of Penetration } \\ k_{C S} & \text { Contact Stiffness } \\ F_{C} & \text { Contact Force }\end{array}$




\section{Chapter 1}

\section{Introduction}

\subsection{Project Motivation}

In recent years, the transportation industry has experienced a growing demand for acoustically optimized systems. Within the automotive sector, investigations into structure-borne noise from tire-road contact [1], fluid flow through a muffler [2], aerodynamic pressures during driving conditions [3], as well as internal combustion engine sounds [4] are some areas being considered to improve the user experience based on acoustics. The sound sources mentioned occur during vehicle operation and are not the first sounds heard by a potential customer. In practice, transient Noise, Vibration and Harshness (NVH) acoustic phenomena such as door closing events are often the first sounds heard by the customer during vehicle operation. This short-duration sound serves two purposes: to inform the driver that the door has been properly closed; and to provide a "first impression" of the vehicle quality to the user. The impression that is made depends on the quality of sound that is produced as well as the customer's subjective interpretation the sound.

Psychoacoustics is a branch of acoustics which studies how a person's interpretation of a sound is influenced, based on characteristics that are intrinsic to the sound [5]. Following psychoacoustic principles, the sound quality generated by door closing events can influence one's perception of the vehicle's overall quality, making it an important criterion in the decision to purchase one vehicle over another. This work, in collaboration with Magna Closures (a division of Magna International Inc), investigated the acoustics of an isolated D21MC automotive door closure system to obtain a better understanding of the radiated sound. 


\subsection{Scope of the Project}

The focus of this work was to investigate different methods of studying the acoustically complex door closure component interaction events and identify the fundamental frequencies composing the acoustic transient response using various post processing methodologies. Experimental results were used to validate a computational model (structural and acoustic) of the door closure system. The experiments focused on the frequency band between $160 \mathrm{~Hz}$ and $5,000 \mathrm{~Hz}$. The lower limit was a result of experimental limitations whereas the upper limit was a result of computational limitations. These limitations are described in further detail in subsequent sections of this report. The objective of this work is to provide insight into the frequency composition of a transient acoustic sound and the interpretation of the sound quality based on psychoacoustic principles. Ultimately, the results from both experimental and computational methods will be used in future work as a means to predict changes in the radiated sound resulting from structural modifications to the door closure mechanism, in addition to creating a more pleasing acoustic response. It should be noted that within this work the terms door latch system, latch, door closure, and door closure mechanism are equivalent.

\subsection{Organization of Thesis}

Chapter One describes a brief introduction to the project, illustrating the motivation and connection to real world applications. It also provides a short overview of the material covered in this work. Chapter Two describes the experimental and computational acoustic terminology; signal processing; and the inner workings of the human ear and its relation to psychoacoustics. This chapter also describes certain attributes regarding standard vehicle latch operation; the types of sounds produced, and a breakdown of the latch components investigated. Chapter Three describes the methods and procedures used to collect transient acoustic data from the D21MC automotive door closure. The results from a continuous wavelet transform (CWT) analysis and sound pressure level (SPL) with respect to $1 / 3$ octave bandwidth are presented along with a discussion of trends. An analysis involving sound pressure metrics is included. Chapter Four describes the methods and procedures used to formulate a simplified computational acoustic 
model of the D21MC door closure within ANSYS Workbench. The procedure involved generating kinematic and dynamic results using ANSYS Rigid Body Dynamics (RBD) and ANSYS Explicit Dynamics (ED) respectively. The results were applied as input variables for the acoustic simulation performed in ANSYS Transient Structural (TS). The results of the computational model are discussed in detail. Chapter 5 provides a summary of the thesis and suggestions for future work. 


\section{Chapter 2}

\section{Literature Review}

This thesis investigates the sound quality of an automotive door closure system using experimental and computational methods. In the literature, there are many different methods used to perform acoustic data collection, post-processing of analog signals, and pre-processing of computational acoustic models. Each method possesses certain advantages and disadvantages over others. Analysis of both past and present investigations elucidated the preferred methods for accurate analysis of transient acoustic phenomena. The methods chosen for this particular study are explained in subsequent sections.

\subsection{Acoustics}

Sound is common in everyday life and needs to be carefully considered in many applications in various fields and disciplines. According to Rossing,

Acoustics has become a broad interdisciplinary field encompassing the academic disciplines of physics, engineering, psychology, speech, audiology, music, architecture, physiology, neuroscience, and others. Among the branches of acoustics are architectural acoustics, physical acoustics, musical acoustics, psychoacoustics, electroacoustics, noise control, shock and vibration, underwater acoustics, speech, physiological acoustics, etc. [6]

An important aspect of acoustic research is the ability to obtain data through non-intrusive means to reveal information that is not easily attainable. In this chapter, aspects of fundamental acoustic terms and principles used in this work are presented and defined.

\subsubsection{Simple Waveform}

A healthy young human ear is capable of hearing frequencies between $20 \mathrm{~Hz}$ and $20 \mathrm{kHz}$ (known as the audible spectrum) and is the frequency band that typically most concerns acoustic engineers [7]. Audible sound is defined as a pressure variation in an elastic medium, typically a fluid such as air or water, that a 
human ear can detect. Common sources of these oscillations are the result of surface vibrations from a structure (vehicle engine) or from turbulent flow within the fluid (vortex shedding). Sound travels in the form or longitudinal waves as shown in Figure 2.1 where the motion of the air particles is parallel to the motion of the propagated energy. The propagation of energy is achieved via compressions and rarefactions of the air particles composing the fluid. From Figure 2.1, it can be understood that the compressions and rarefactions present in the longitudinal wave coincide with positive and negative deviations from atmospheric pressure in the transverse wave respectively [8]. Within this work, recorded sound pressure is presented in the form of transverse waveforms.

(a)
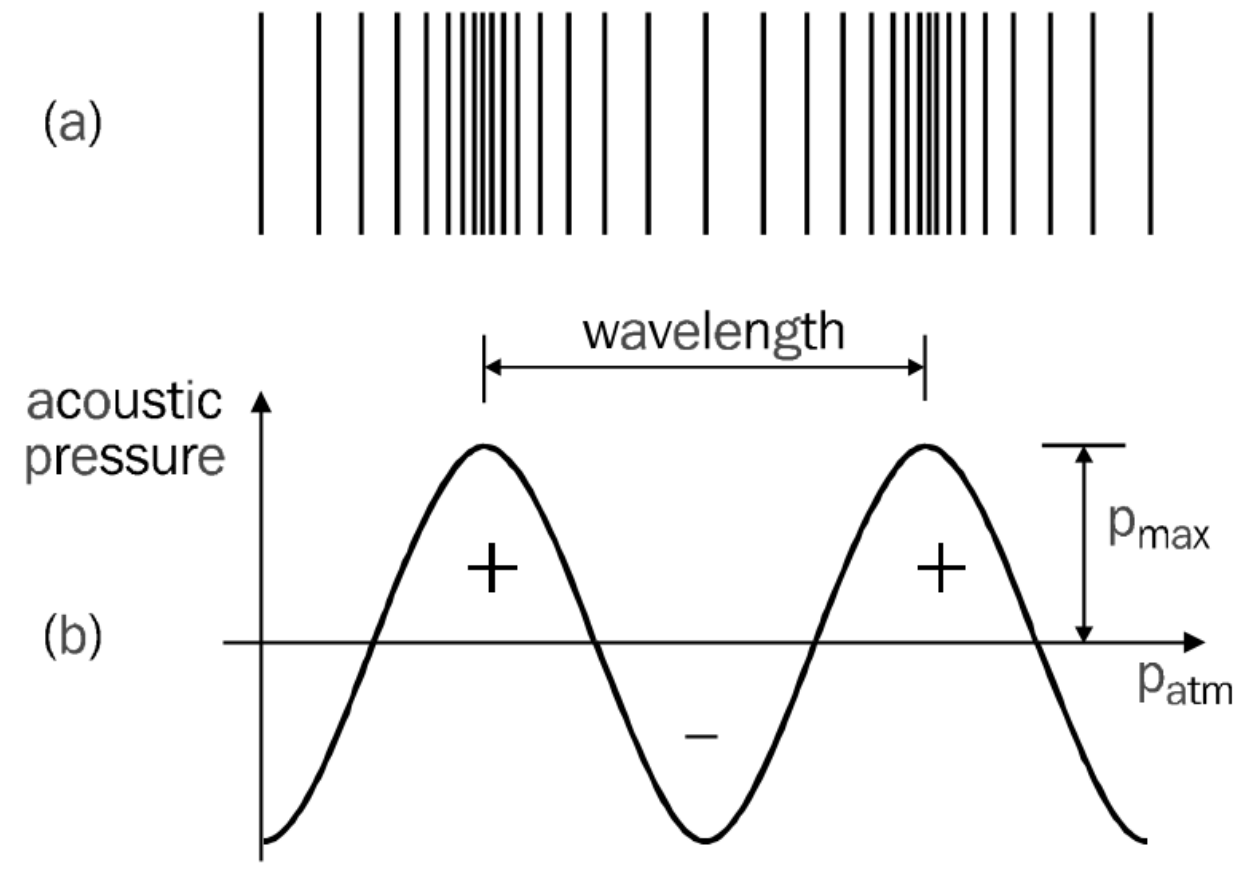

Figure 2.1: a) Longitudinal waveform. b) Transverse waveform [8].

The literature defines wavelength $(\lambda)$ as the distance travelled by the sound wave during one cycle. The frequency $(f)$ of the waveform, expressed in $\operatorname{Hertz}(\mathrm{Hz})$, is defined as the number of successive cycles per second $(s)$. The wavelength of a waveform is inversely proportional to its frequency. 


\subsubsection{Complex Harmonic and Transient Waveforms}

Sounds can be composed of a single frequency (pure tone) or multiple frequencies depending on the nature of the sound source, the method by which the sound is generated, and the propagation path the wave travels to reach the receiver. A large and growing body of literature has investigated the sounds produced by vehicles, machinery, and instruments. These investigations found that common everyday sounds typically consist of multiple frequencies that are superimposed to form single complex waveforms [7].

One study by Petersen examined waveforms generated by various musical instruments in an effort to identify certain trends of the recorded sounds via sound pressure and spectrogram analysis [9]. Each instrument played the same note; a Middle A which has a fundamental frequency of $440 \mathrm{~Hz}$. The recorded waveforms and spectral plots are displayed in Figure 2.2. It was discovered that each recorded waveform had virtually identical fundamental frequencies of $440 \mathrm{~Hz}$. This can be seen by the wavelength of each sample being almost identical. The tuning fork produced a simple waveform as expected; much like the one described in Figure 2.2. However, the other instruments produced complex pressure variations within the waveform indicating the presence of additional weaker frequencies superimposed within the sample. These additional frequencies are the harmonic frequencies which affect the timbre, or sound quality of the instrument. This variation in timbre allows humans to identify instruments based solely on the sound they emit. A spectral analysis plot for each instrument was produced using a Fourier transform. As a result, the spectrum identified the fundamental frequency of the sample symbolized by the largest peak, as well as each harmonic frequency [9].

Drawing from the findings of this work, Petersen was able to show that musical instruments, which are capable of producing fundamental frequencies, also exhibit harmonic frequencies within the generated sound. It was suggested that these weaker harmonic frequencies can have an impact on the sound quality [10]. The mention of these findings from Petersen was to clarify that not all sounds take the form of a 
simple sinusoid represented by Figure 2.1b; waveforms can become quite complex and can contain multiple superimposed frequencies which can affect the sound quality in numerous ways.
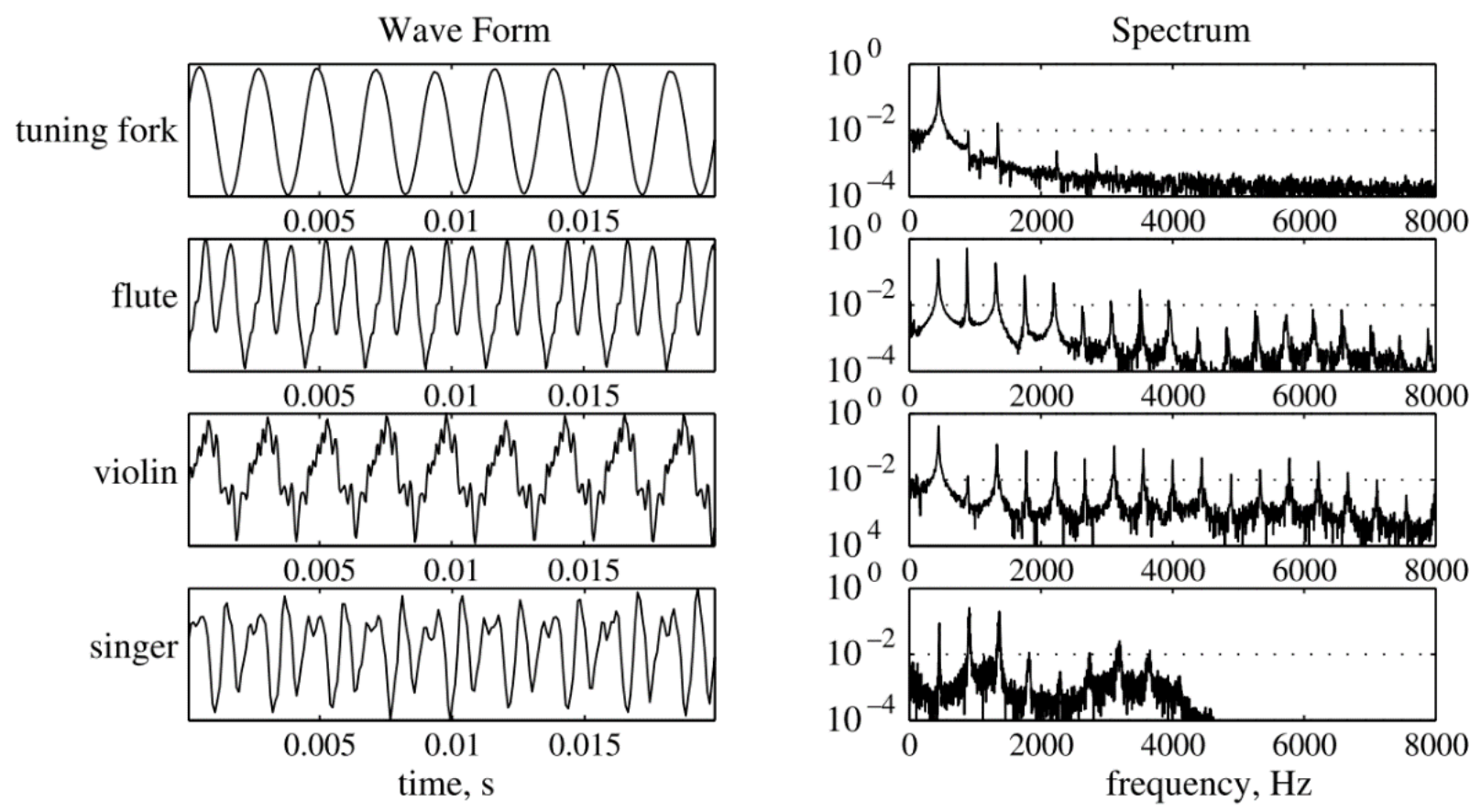

Figure 2.2: Complex waveforms and corresponding spectrogram of various musical instruments [9].

A considerable amount of literature has been published on harmonic acoustic sources such as the ones studied by Petersen. Harmonic sources are defined as objects or matter that produce pressure variations in an acoustic media at a constant frequency, or a series of superimposed frequencies, for a long duration of time [11]. As a generalization, sounds can be classified as either harmonic (Figure 2.2), or impulsive (transient). Transient sources are described as objects or matter that produce pressure variations in an acoustic media consisting of a large, sudden deviation from ambient pressure which then decays over time. Typically, transient sounds are no longer than approximately 1 second in duration [12]. There has been little quantitative analysis investigating the frequency composition of transient acoustic sources and phenomena. Therefore, this study provides an opportunity to advance the understanding of the impulsive sounds in general and the impulsive sounds generated by automotive door closure systems in particular. 


\subsection{Vehicle Door Closure System Acoustics}

\subsubsection{Objective Study}

In automotive engineering acoustic radiation analysis and structural vibration analysis are powerful tools used by acoustic and design engineers [13]. By collecting sound information of automotive door closures, inherent qualities if the sound source can be determined. This formed the central focus of a study by Beker et al. in which the authors performed an objective study to examine the NVH phenomena of a door closing event. An objective study involves the culmination of results and observable trends based on factual, quantitative data. For this particular study, two door closure events from two different vehicle models were investigated. Figure 2.3 displays a transient sound waveform from a vehicle door closure. For the purpose of this report, the two sounds are denoted as Subject A and Subject B. At the start of the investigation, it is not expressed to the reader the make and model of either the door closure or the vehicle it is affixed to. The authors collected sound pressure measurements and analyzed the data using two signal processing methods: Short-Time Fourier Transformation (STFT) as well as a Continuous Wavelet Transformation (CWT).

The secondary objective of this work was to report which signal processing method performed better at analyzing the frequency composition of the impulsive signal. The findings indicate the "Wavelet Analysis reveals the details of the frequency content of the transient signal more clearly than the Short-Time Fourier Transform... For the analysis of transient door closure sounds the usual Fourier transform is not suitable. The time content of the signal is lost during the transformation process" [14]. The advantage CWT has over STFT is the ability to adapt the "windowing" feature of the function in order to obtain an increased resolution in the time or frequency domain when appropriate [15]. 


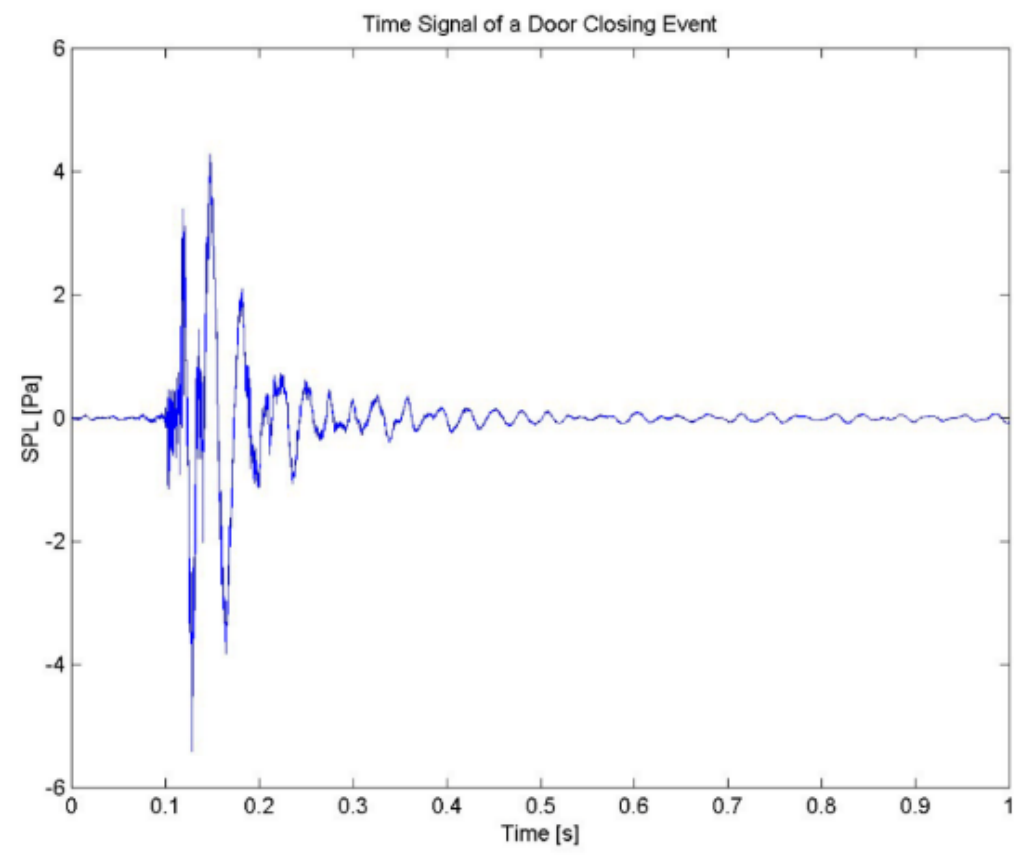

\section{Figure 2.3: Transient acoustic waveform of a door closure sound used in the objective study [14].}

An interesting finding was that the subjective evaluation (an analysis based on personal feelings and judgement) identified Subject A as the more pleasurable sound as opposed to the sound generated by Subject B. Subject A was described as being "tremendously dark... This type of sound is associated with upper class vehicles and a high expectation of quality by the customer" [14]. Conversely, Subject B sounded "tinny and light... The subjective perception allocates this sound to a low-budget vehicle with a lower level of quality" [14].

The reason for this outcome was the influence of dominant frequencies within the aural response. Subject A was praised for its sound because it mainly comprised of lower frequency content, primarily within the $50 \mathrm{~Hz}-500 \mathrm{~Hz}$ bandwidth which proceeded to resonate for a longer duration compared to the frequencies above $500 \mathrm{~Hz}$. Similarly, Subject B was criticized for its less than satisfactory response resulting from dominating higher frequencies, $750 \mathrm{~Hz}$ and above, which resonated for a longer duration. Both trends involving frequency composition can be seen in Figure 2.4. 

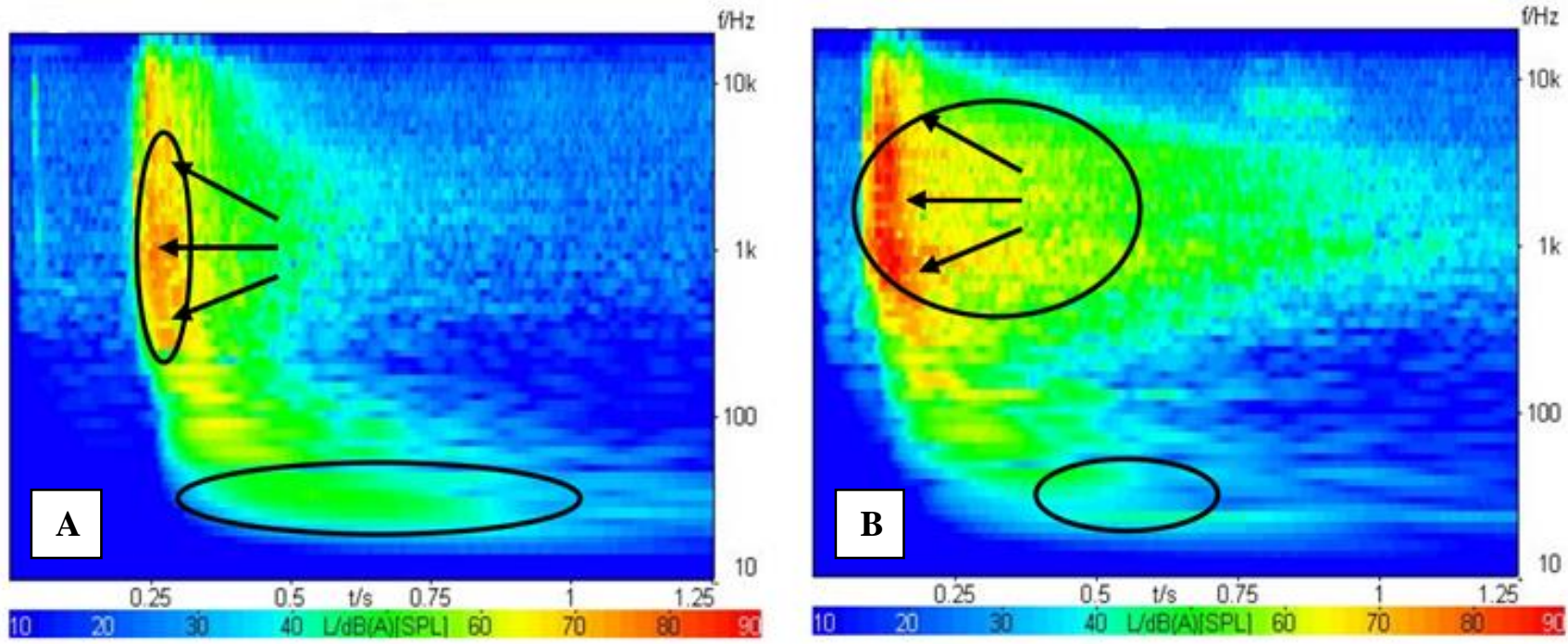

Figure 2.4: CWT of the recorded door sounds of Subject A and Subject B respectfully. The colour bar represents intensity of normalized magnitude [14].

The study performed by Becker et al. makes a major contribution to the area of door closure acoustics by demonstrating the suggested effect it has on the perception of the vehicle as a whole; the prescribed method of analyzing a complex waveform; as well as providing a reasonable analysis of the trends observed in the CWT plots. As a result of the proven accuracy and reliably of the CWT, the method was adopted to analyze the D21MC latch. A detailed description of the CWT method, as well as the produced results, is provided in a subsequent chapter.

\subsubsection{Subjective Study}

In a similar vein, this view is supported by other authors who have investigated the aural response of transient pressure waves from door closures. A significant addition to this area of study can be seen in the works from Sonoko Kuwano and associated research fellows whose academic accomplishments have spanned many years with many contributions in the field of acoustics. Their research is primarily focused on the subjective impression of temporal impacts and the mental image that is contrived in the mind of the consumer [16]. Door closure sounds have been a topic of many research papers. One particular study 
involved recordings of 11 door closure sounds (stimuli) from vehicles categorized as: luxurious sedan, expensive sports car, economic sedan, pick-up truck and "other". Participants of both German and Japanese descent were used in the study where they were asked to subjectively quantify each stimulus based on specific acoustic descriptors. An example of the results from this procedure can be seen in Figure 2.5. The results in Figure 2.5 show excellent constancy between the two test groups with a coefficient of correlation (CoC) of 0.946 .

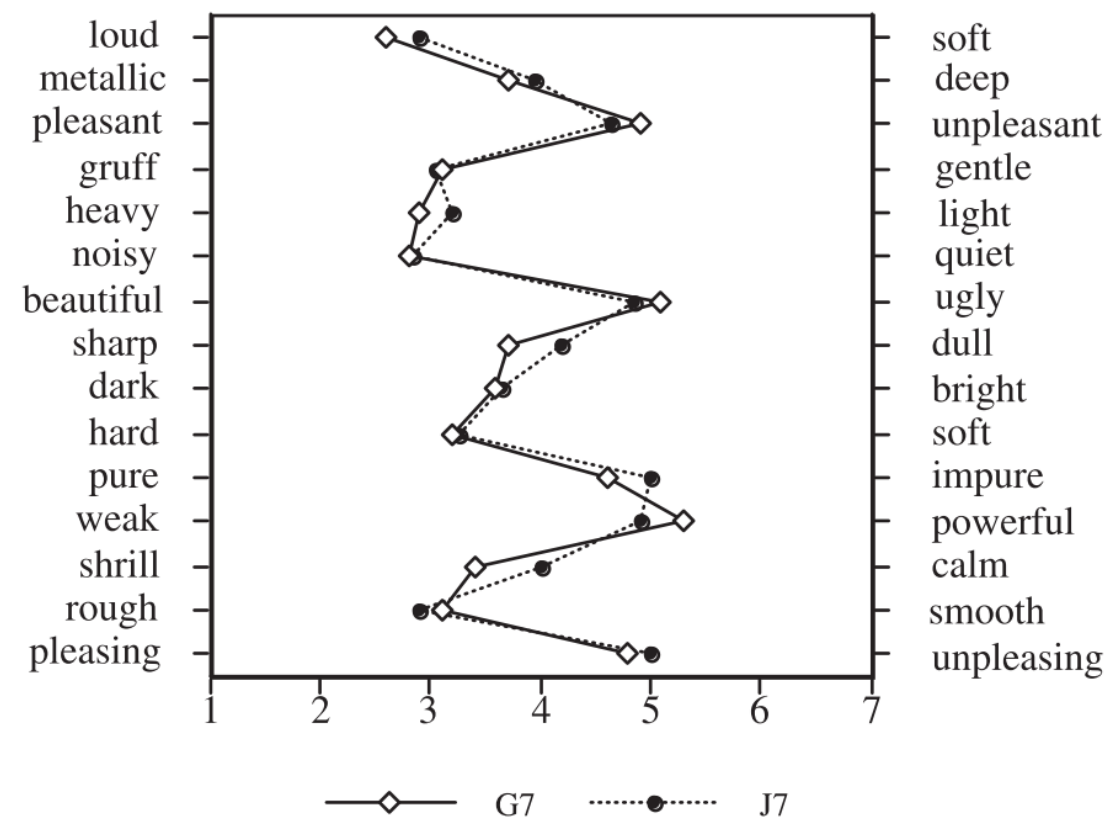

Figure 2.5: Subjective results of one stimuli based on acoustic descriptors. Diamond and circle points refer to the German and Japanese participants respectively [16].

In addition, the participants were instructed to envision a vehicle type that best affiliates with the stimuli presented. The stimuli were presented in clusters. The results from cluster 1 "indicate that this sound was perceived as being deep, pleasant, gentle and heavy. This suggests that cluster 1 is a group of pleasant sounds. The images from the sounds which belong to cluster 1 were associated with luxurious sedan by high percentages..." [16].The sounds associated with cluster 2 "indicate that this sound was perceived as being metallic, unpleasant, gruff and light. This suggests that cluster 2 is a group of unpleasant sounds. 
The images of the sounds which belong to cluster 2 were associated with economy sedan by high percentages" [16].

The consistency in the results from the two test groups strongly suggest that the human ear is capable of identifying characteristics within the sound that are more pleasurable to hear. The results from this study seem to agree with the results of the Becker study such that sounds comprised of higher frequencies, typically $1.5-2 \mathrm{kHz}$ and above, are generally not pleasing to hear. Notice how this statement is a generalization, as every human has a different mental image of sounds that are considered acceptable. This concept is discussed further in a succeeding section. This study aims to contribute to this growing area of research by exploring the significance of door closure sounds in modern vehicle design.

\subsection{D21MC Automotive Door Closure System}

The mechanism used for this analysis was the D21MC automotive door closure system designed and fabricated by Magna Closures (a division of Magna International Inc.) in Newmarket, Ontario, Canada. Images depicting the full assembly of the mechanism in isometric, side and frontal view can be seen in Figure 2.6. A majority of the parts are imbedded within the assembly. The latch system is comprised of 27 interconnecting parts, each with their own function and material properties. However, there is a subset of components tasked with locking and unlocking the mechanism during operation. These parts were the main focus of this examination. 

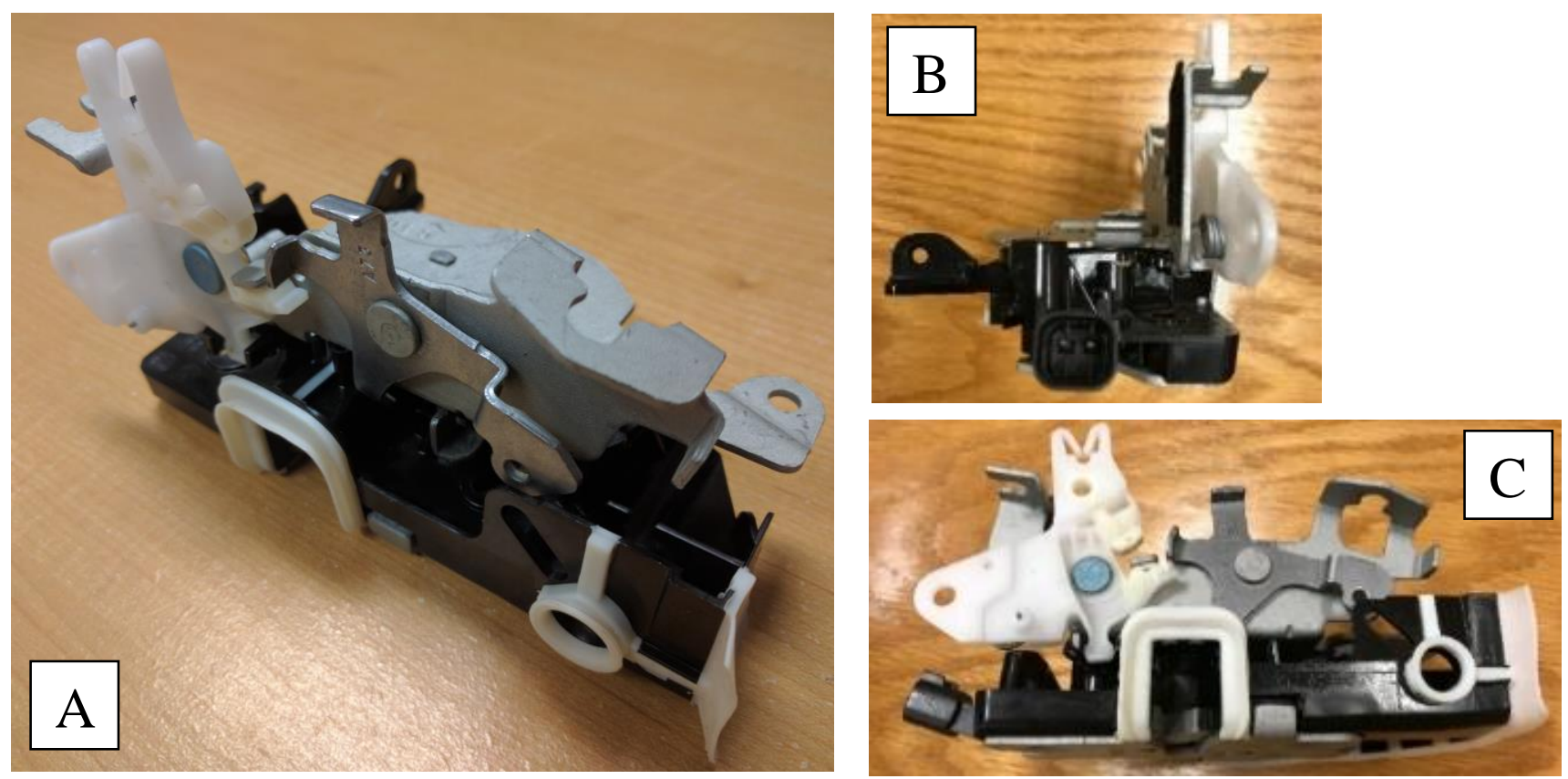

Figure 2.6: D21MC Latch Assembly A) Isometric view, B) Side view, C) Front view.

The three main components of the latch used in this investigation were the striker, ratchet, and pawl. Engineering models of the striker, ratchet and pawl are displayed in Figure 2.7. This particular door latch model is denoted as a Primary Door Latch. This designation is given to latches equipped with both a fully latched position and a secondary latched position. A secondary latched position refers to the coupling condition that retains the door in a partially closed position [17]. If the latch was in the partially closed position during regular use, this security feature still ensures the safety of the vehicle occupants in the event of an accident. Figure 2.7 is a depiction of the striker, ratchet and pawl in the fully un-latched position. It should be noted that the other latch components (frame, housing, lock lever, etc.) are not included for ease of viewing. 


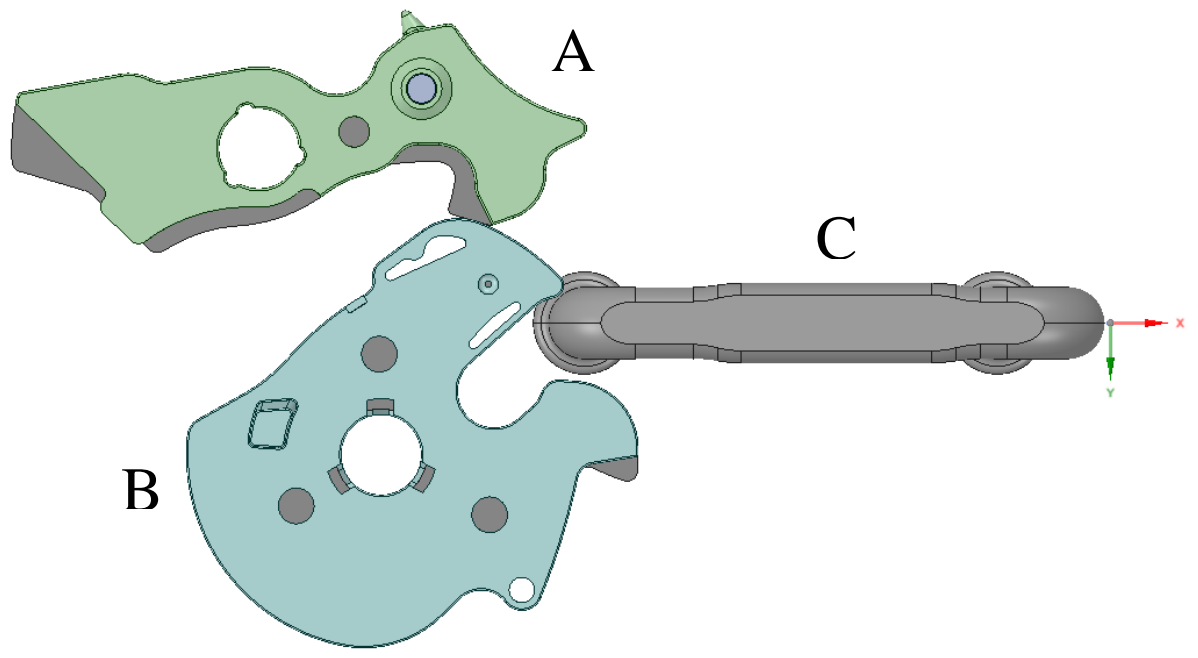

Figure 2.7: A) Pawl and pawl encapsulation. B) Ratchet and ratchet encapsulation. C) Striker

In a typical automotive door assembly, the door is attached to the vehicle A Pillar via a hinge mechanism. The door closure mechanism is imbedded within the door assembly on the opposite end from the hinge. Generally, the striker is affixed to the lower portion of the vehicle B Pillar.

As an automotive door is forced to close, the striker meets the fork-bolt of the ratchet. The fork-bolt is the first contact point of the latch engagement as shown in Figure 2.7. The force from the striker causes the ratchet to rotate in the direction known as the fork-bolt closing direction until the pawl shifts past the fork-bolt. Should the ratchet cease to rotate at this point, the latch would be denoted as being in the secondary latched position.

In normal latch operation, the striker will continue to rotate the ratchet until the pawl fully engages with the ratchet, thus locking the mechanism and preventing the door from opening [17]. This coupling condition is known as the fully latched position. The progression from un-latched position to fully latched position is depicted in Figure 2.8. 

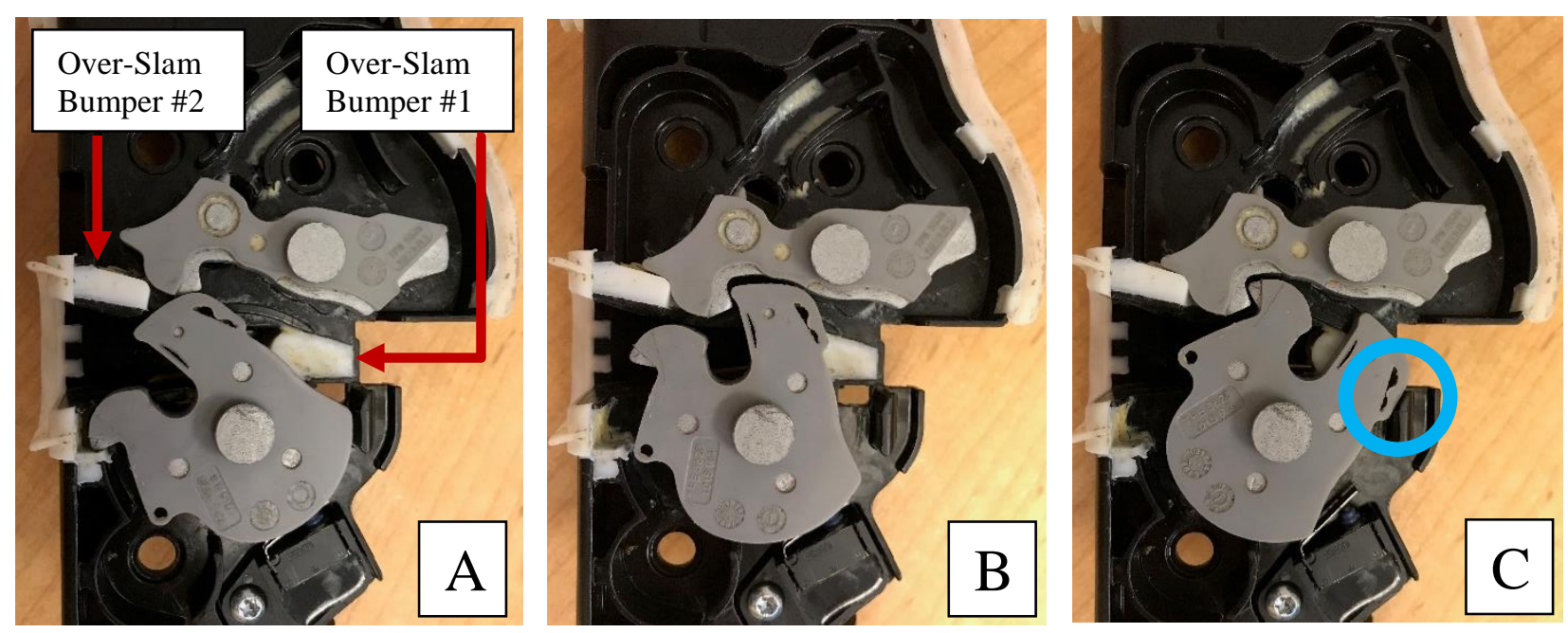

Figure 2.8: Latch locking progression. A) Fully un-latched position. B) Secondary latched position. C) Fully latched position.

As the door closure transitions from the fully un-latched to the fully latched position, the kinetic energy of the striker and the pawl are both absorbed by "over-slam" bumpers. These bumpers, comprised of a thermoplastic vulcanizate known as Santoprene, are meant to protect the latch components from potential damage. The polymer bumpers can be seen in Figure 2.8A denoted by the red arrows. The ratchet encapsulation incorporates its own bumper system which serves the same function as the over-slam bumpers. Figure $2.8 \mathrm{C}$ shows the ratchet bumper denoted by a blue circle. Rather than impacting a thermoplastic vulcanizate, the bumper simply impacts a portion of the latch housing. Knowledge of these impact events is critical for the understanding of which components have a greater influence on the complex acoustic response of the system. This is further discussed in a subsequent chapter.

The striker, ratchet and pawl were fabricated using alloy steel. An additional part denoted as the pawl pin was also fabricated using the same steel alloy. The purpose of the pin is to allow the pawl to disengage with the ratchet and unlock the mechanism. Both the ratchet and the pawl are encased in a durable thermoplastic polymer encapsulation. As previously mentioned, the over-slam bumpers were made of Santoprene which is a complex polymer mixture known for its durability. Table 2.1 describes the material properties of each of the main components experiencing impact events during the locking and unlocking 
procedure. Knowledge of these materials and their relative properties was vital for the computational portion of this work.

Table 2.1: Material properties of the latch components used in door locking operation (provided by Magna Closures).

\begin{tabular}{|c|c|c|c|c|}
\hline Part Name & Material & $\begin{array}{l}\rho, \text { Density } \\
{\left[\mathbf{k g} / \mathbf{m}^{3}\right]}\end{array}$ & $\begin{array}{c}E, \text { Elastic } \\
\text { Modulus }[\mathrm{MPa}]\end{array}$ & Poisson's Ratio \\
\hline $\begin{array}{l}\text { Pawl Insert, } \\
\text { Ratchet Insert, } \\
\text { Striker }\end{array}$ & $\begin{array}{c}\text { Alloy Steel (UNS } \\
\text { G41300) }\end{array}$ & 7,850 & 205,000 & 0.29 \\
\hline $\begin{array}{c}\text { Pawl } \\
\text { Encapsulation }\end{array}$ & $\begin{array}{l}\text { TPE Hytrel } \\
\text { G3548 }\end{array}$ & 1,150 & 166.7 & 0.45 \\
\hline $\begin{array}{c}\text { Ratchet } \\
\text { Encapsulation }\end{array}$ & TPE Hytrel 5526 & 1,190 & 136.2 & 0.45 \\
\hline Housing & POM Delrin 100P & 1,420 & $1,817.1$ & 0.35 \\
\hline $\begin{array}{c}\text { Over-Slam } \\
\text { Bumper \#1 and } \\
\# 2\end{array}$ & TPV Santoprene & 970 & 18 & 0.493 \\
\hline
\end{tabular}

\subsection{Physiology of the Human Ear}

Included in this section is a discussion on the inner workings of the human ear and its ability to interpret environmental pressures as comprehensive audio signals. The ability for a human to hear is simple in concept, but beyond the surface, the entire procedure is quite complex. The human ear is segmented into 3 sections: The Outer Ear, the Middle Ear, and the Inner Ear [18]. These sections are shown in Figure 2.9 [19]. The Outer Ear is comprised of cartilage and tissue known as the Pinna or Auricle. It contains ridges and depressions that funnel sound waves into the Concha which acts as the entrance way into to the External Acoustic Meatus, colloquially known as the ear canal. At the other end of the canal is the Tympanic Membrane or eardrum. This circular membrane signifies the boundary between the Outer Ear 
and the Middle Ear. The extremely sensitive membrane is responsible for converting acoustic pressure variations, like the one outlined in Figure 2.1a, into mechanical vibrations [18].

\section{Anatomy of the Ear}

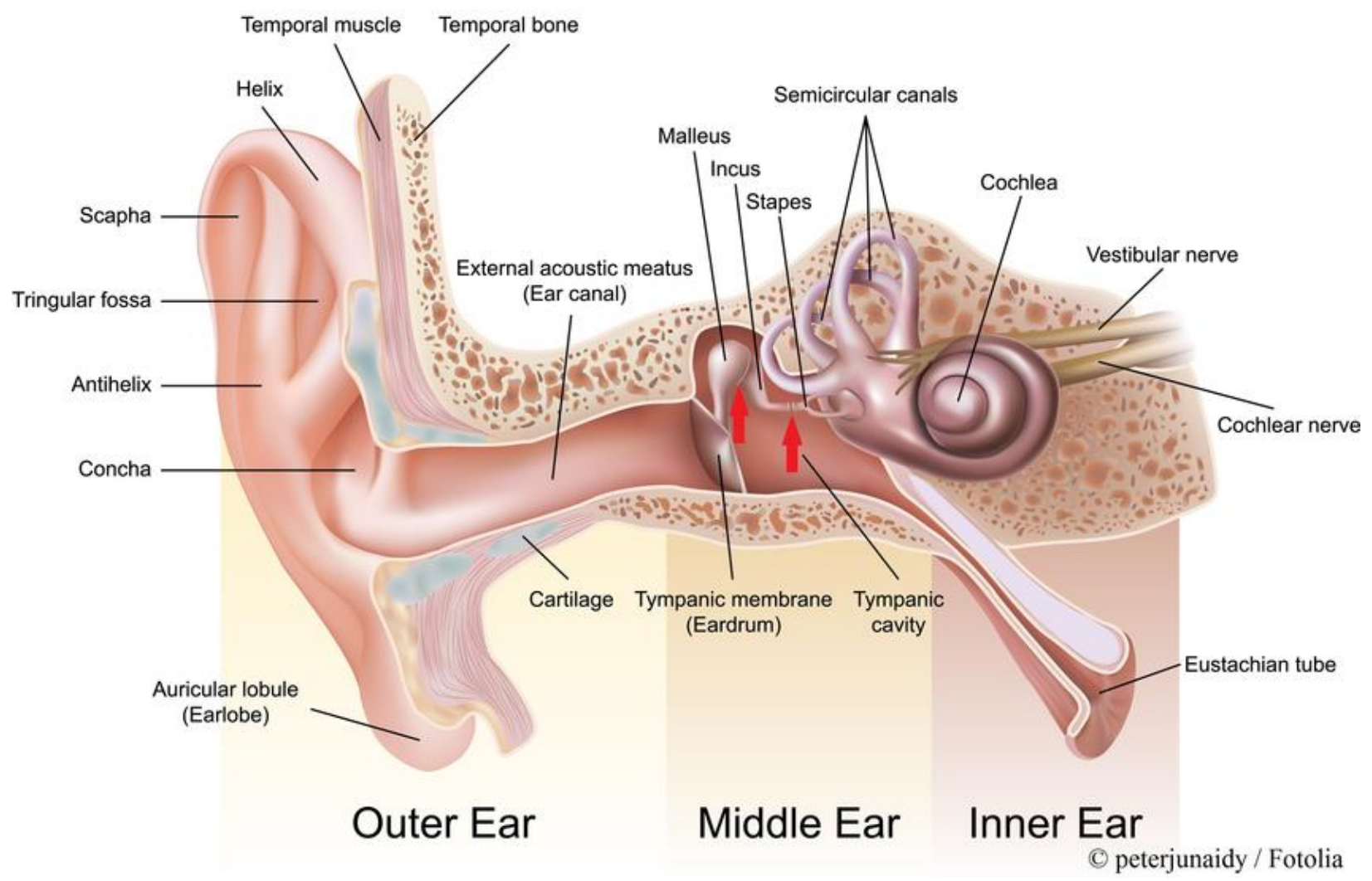

Figure 2.9: The anatomy of the human ear [19].

The Middle Ear, also known as the Tympanic Cavity, serves two purposes: to transmit the mechanical vibrations of the tympanic membrane to the Cochlea (Inner Ear), as well as to protect the Inner Ear from extreme sound pressures [18]. The vibrations are transmitted via 3 bones denoted as the Auditory Ossicles within the Middle Ear. These bones are the Malleus, Incus and Stapes. As the Tympanic membrane oscillates, it causes the Malleus to move which in turn causes the Incus and Stapes to move as well. The bones of the Ossicular Chain connect at flexible junctions denoted by the red arrows in Figure 2.9. This linkage system amplifies the mechanical vibrations in order to overcome the increased 
impedance to motion due to the working fluid within the Inner Ear. A mathematical model showcasing the amplification procedure is explained in detail in the work by Howard et al. [18]. The amplified vibration signal exits the Middle Ear via the Stapes. The Stapes conducts these vibrations to another membrane known as the Superior Oval Window which acts as the boundary between the Middle Ear and Inner Ear.

The Inner Ear is a complex labyrinth which is divided into two regions, each with its own specific function. The first region converts the mechanical vibrations transmitted by the Middle Ear into electrical signals that the brain can understand. The second region assists in maintaining the body's state of equilibrium (Vestibular System). In this section only the former is discussed. As previously mentioned, the oval window membrane connects the Stapes to the Cochlea, a "snail-like" fluid filled tubular structure constructed of various membranes and vestibular channels. The membrane known as the Basilar Membrane contains over 20,000 flexible fibers that resonate at specific frequencies (pitch) within the bandwidth of human hearing. As the Oval Window moves in tandem with the Stapes, the membrane oscillates the special fluid in the Cochlea which in turn causes the fibers of the specific sections of the Basilar membrane to vibrate depending on the pitch of the sound entering the ear. These resonating fibers initiate a reaction in the adjacent membrane, known as the Organ or Corti, which is composed of numerous hair like cells. When triggered, these cells send electrical impulses via the Cochlear Nerve to the brain. The brain can then identify the frequency of the sound depending on the section hair cells triggered by the resonating fibers [18].

\subsection{Psychoacoustics}

Sound affects humans in many different ways. Treasure demonstrates four distinct categories in which sound can affect humans; Physiological, Psychological, Behavioural and Cognitively [20]. The physiological effects of sound can affect hormone secretion within the body, breathing and heartrate. Examples of this are the sudden shock felt resulting from an alarm sounding or the relaxed state of mind that typically results from peaceful sounds of a tide rushing in and out at the beach [20]. Psychological 
effects involve changing our emotional state based on the type of sound being heard. Music is the most powerful form of sound that can affect our overall emotional state [20]. In musical theory, major chords are generally perceived as "happy" whereas minor chords are perceived as "sad" [21]. Similarly, sounds can affect how humans behave. For example, loud and heavy music found at a rock concert typically motivates more energetic behaviour. The last category expresses the cognitive effects of sound. According to Treasure, this is the category business and office work environments should be most concerned with because it expresses how sounds can affect a one's ability to work productively. Treasure indicates that noisy environments can reduce levels of productivity by up to $66 \%$ [20].

Psychologically, the perception of sound differs for each individual. It is generally accepted that humans associate unwanted or unpleasant sounds as noise and seek ways to mitigate the annoyance by applying modifications to the sound source (vibration isolation), sound path (barriers) or sound receiver (location) [7]. Psychoacoustics is a branch of acoustics which studies how a person's interpretation of a sound is influenced by certain inherent qualities of the sound [5]. The set of parameters used to characterize sounds in the field of psychoacoustics are denoted as Sound Quality Metrics. Such metrics include loudness, sharpness, roughness and tonality.

It is common knowledge that many consumer goods produce sounds either on their own or induced by the application of an external force. The literature suggests that there is a relationship between the discerned overall quality of a product and the sound that radiates from it [18][12]. It is a widely held view that loudness and sharpness play a significant role in the quality of sound [22]. According to a statute created by the Acoustical Society of America (ASA), loudness is defined as the "attribute of auditory sensation in terms of which sounds may be ordered on a scale extending from soft to loud. Loudness depends primarily upon the sound pressure although it also depends upon the frequency, waveform, and duration of the sound" [23]. Sharpness is characterized as the measure of high frequency content present in a sound [24]. 


\subsubsection{Sound Quality Metrics: Sharpness and Loudness}

Returning to a previously mentioned subjective study, Kuwano et al. provide a graphic showing acoustic profiling of various stimuli used in the investigation. This graphic is shown in Figure 2.10. The acoustic descriptor scales relating to loudness were assumed to be "loud-soft", "noisy-quiet" and "hard-soft" ranging between 1-7 (4 being neutral). In the study it was found that stimuli 3 and 6 were less preferred compared to stimuli 8 and 9. Stimuli 3 and 6 were identified as being 'harder', 'noisier' and 'louder'. However, it is interesting to note that stimuli 9 achieved a loudness level almost identical to the less preferred sounds, whereas stimuli 8 was viewed as being the most 'soft'. Stimuli 9 was still preferred even though it was perceived as a 'louder' sound compared to stimuli 8 . The data appears to support the assumption that increasing the loudness of a sound has a weak correlation with the overall impression of the sound.

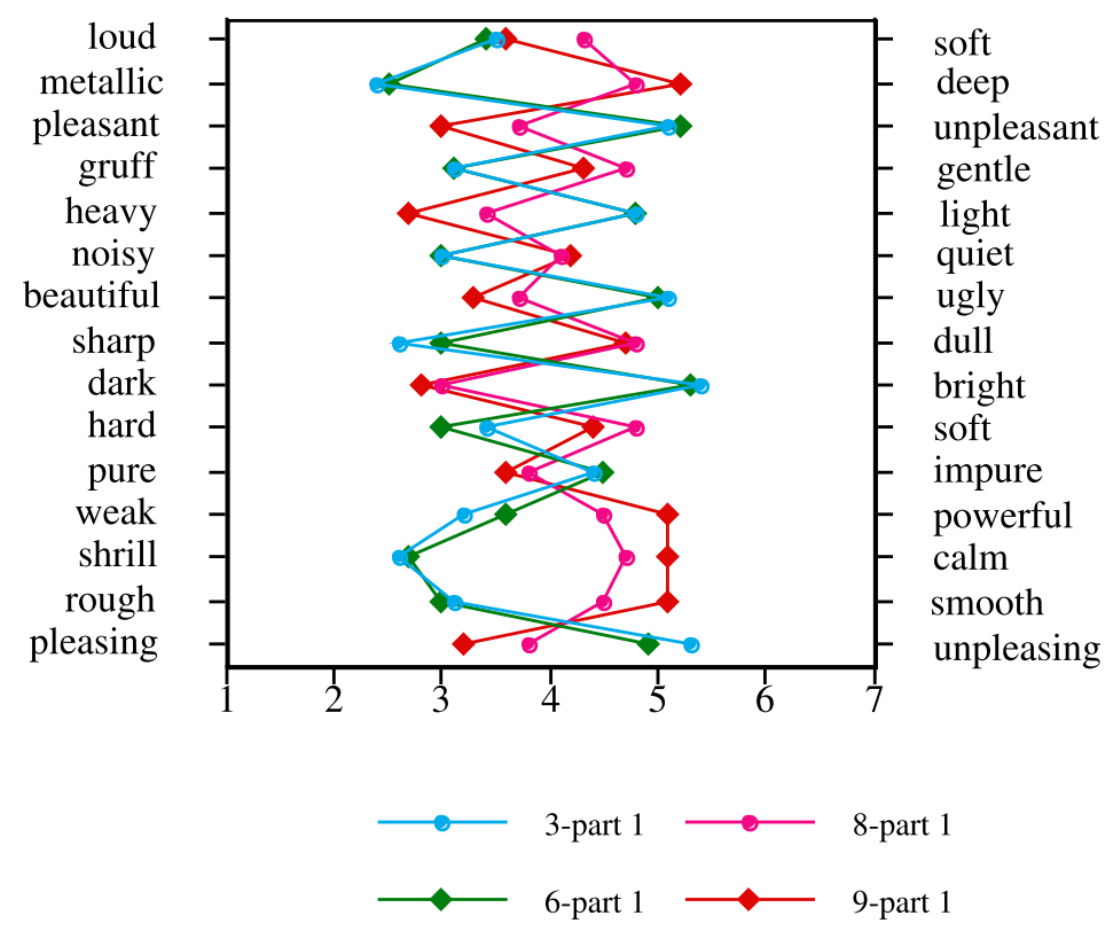

Figure 2.10: Subjective evaluation between four vehicle door stimuli [25]. 
Figure 2.10 also shows acoustic descriptor scales relating to loudness such as "metallic-deep", "sharpdull", "dark-bright". By inspection, the findings are consistent with other research presented in this chapter, which suggest sounds that possess 'deeper' and 'darker' tones are more preferred. The graphic displays a clear consensus amongst the reviewers regarding the placement of each stimuli within each scale. This supports the assumption found in past studies that sharpness has a greater influence on the sound quality of a door latch compared to loudness [25].

Based on the literature examples mentioned, it can be assumed that individuals tend to prefer sounds comprised of lower frequencies (less sharp) over sounds composed of higher frequencies (more sharp) $[14][16][26]$. The research presented in this thesis attempts to compliment the aforementioned studies by applying similar procedures and methodologies towards the analysis of the D21MC latch. However, it should be mentioned that this study was performed on the basis of objective analysis while incorporating certain aspects of subjective analysis and psychoacoustics to investigate the frequency composition of the acoustic phenomena.

\subsection{Computational Acoustic Processing}

As seen from previous sections, several studies have explored the reliability of experimental acoustics regarding automotive door closures (Becker et al.; Kuwano et al.). However, there is a lack of documented research into computational predictions of automotive door closure sounds. Therefore, this paper attempts to develop a method in which computational acoustic simulations can be conducted and validated against experimental results. Knowledge from this study is expected to assist researchers and designers in future endeavors to predict the aural response of a door closure resulting from structural or acoustical modifications.

Previous computational acoustic work has demonstrated that the dynamic analysis of individual components of a structural model is recommended in order to identify which subsystems have a greater influence on overall sound quality. An example of one such study was the structural and acoustic 
behavioural analysis of a washing machine carried out by Beckmann et al [27]. Von Estorff et al.

provided a summary, translated into English, of the work presented in Beckmann et al.; Von Estorff was a contributor to the project. Due to the harmonic nature of the excitation forces and the complex assembly of mechanical components and the acoustic insulating material within the washer lining, it was necessary to separate the resulting vibro-acoustic analysis into multiple steps. According to Von Estorff,

....in order to obtain a generally applicable model, different computation procedures had to be combined with measured values. The final numerical model is based on a combination of the conventional finite element method to determine structural vibrations, multi-body- dynamic (MBD) algorithms to analyze the machine dynamics, and the boundary element method to compute the radiated acoustic waves. The dynamic characteristics of single components of the structural model have been specifically investigated by accompanying measurements and it took several steps until the complete model had been assembled [28].

As a generalization, the first step involved the identification of the washer subsystems which have the most influence on sound generation. The following involved a computational analysis to determine the resulting structural dynamics of the assembly. With the knowledge of the external/internal harmonic forces and the predicted behaviour of the assembly known, a computational acoustic analysis was performed. Due to the complexity of the model, a Boundary Element Method (BEM) was adopted. A brief description of the advantages and disadvantages of FEM and BEM is included in a subsequent chapter. The method of using collected measurements from real life structures as input parameters in a computational model is not uncommon. As previously mentioned, a similar procedure was performed for the analysis of tire-road noise during driving conditions [29] as well as wind noise from turbulent flow [3].

By drawing on the concept of simplifying a large complex system into smaller subsystems, Beckmann was able to show that a reliable and accurate computational model to predict the radiating acoustic field can be achieved. The results from this study are presented in Figure 2.11 where a comparison of the Sound Power Level versus 1/3 Octave Bandwidth between the experimental and computational results are 
displayed. Overall the agreement is considered to be "excellent" by Estorff, proving the effectiveness of the method.

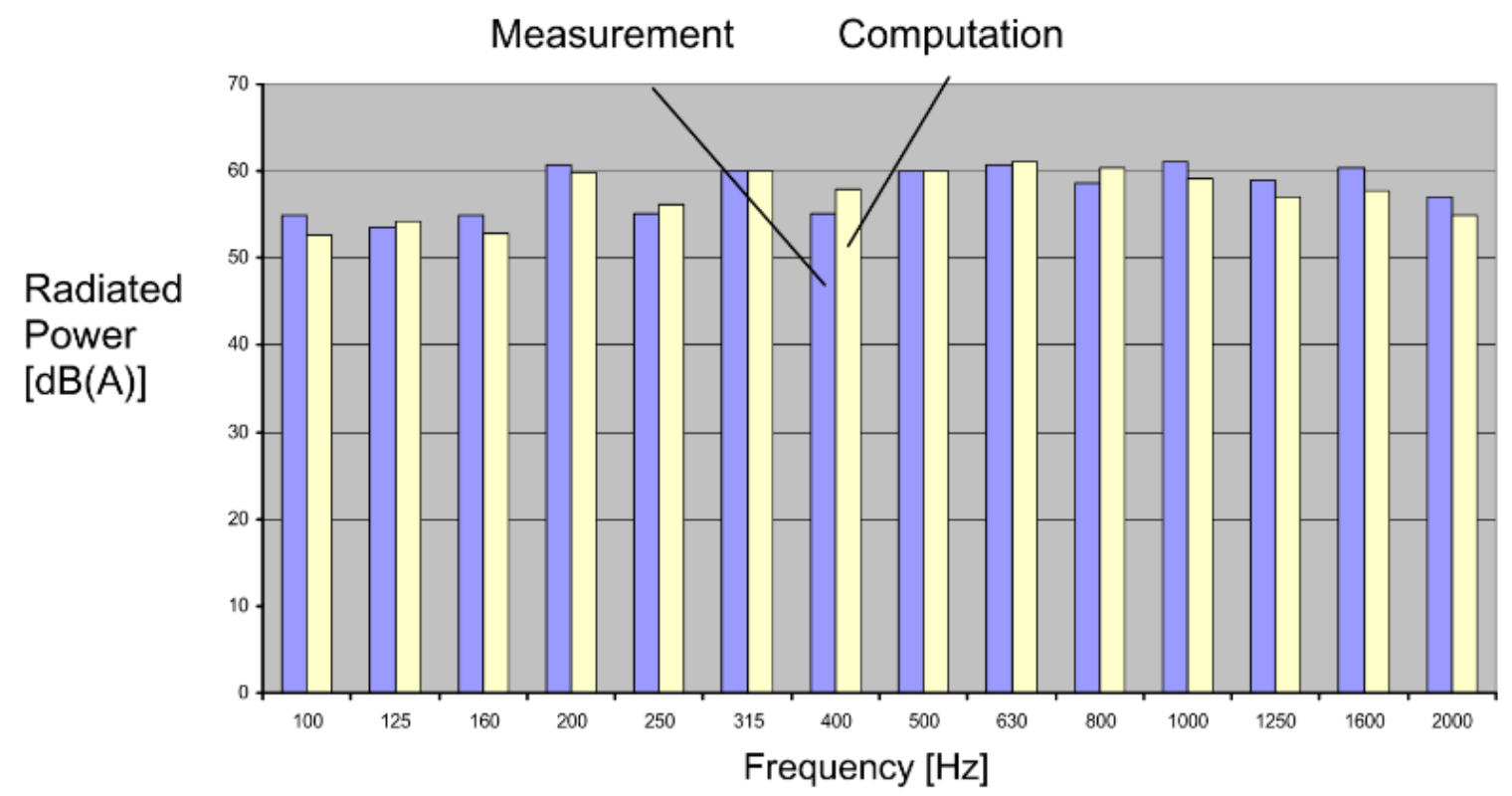

Figure 2.11: Comparison of the experimental and computational results of the washing machine study [28].

Given the evidence presented, the methodology of identifying components which contribute most to the radiated sound was adopted for the D21MC study. Additionally, the data presentation method in Figure 2.11 was shown to be an excellent method of conveying and comparing the frequency composition of the radiated sound. Therefore, this quantitative approach was also employed in the current study. In addition, a qualitative analysis of Sound Pressure Level verses 1/3 Octave Bandwidth is presented and discussed.

\subsection{Summary}

In conclusion, a short introduction to acoustic waveform types and properties has been presented. Acoustic radiation was described as longitudinal pressure variations within a fluid media which transmits 
energy and sound quality characteristics. Sound is an important, but often overlooked, aspect of everyday life. It can be used as a non-intrusive method of providing information about a product or structure that is not otherwise easily attainable. There are many examples of objective and subjective analyses investigating the acoustic phenomena of automotive door closure systems with observable trends. These trends elucidate which sounds are preferred over others and the potential reasoning for this preference. A branch of acoustics known as psychoacoustics investigates human psychological perception of a sound based on the qualities of the sound. It is suggested that the frequency composition influences this perception. For example, a sound with a strong contribution from higher frequencies (higher pitch) is less preferred compared to a sound dominated by lower frequencies.

Psychoacoustics aims to strengthen the general understanding of what makes an individual prefer one sound over another. These methods have the potential to be applied in many aspects of engineering design to achieve a desired sound. As a result, this study aims to contribute to this growing area of research by exploring the radiating acoustic phenomena of an automotive door closure during operation. 


\section{Chapter 3}

\section{Experimental Procedures and Data Analysis}

\subsection{Experimental Background}

According to the literature, the ideal test condition for performing acoustic measurements is a fully outdoor environment free from hard surfaces and obstructions [7][11][30]. This condition is known as a free-field (FF) environment and follows the conventional 6db sound pressure level (SPL) reduction per doubling of the distance which is ideal for sound measurements [30][6]. Although it is ideal, it is highly impractical due to the various types of sporadic noise pollution from most outdoor environments. This chapter outlines the steps taken to collect accurate acoustic experimental data for the D21MC latch as well as identify and discuss trends present. The contents of this chapter have been adapted from a peerreviewed conference paper entitled "Experimental and Computational Methods for Investigating Automotive Door Closure Sounds"[31].

\subsection{Experimental Equipment and Test Setup}

\subsubsection{Semi-Anechoic Chamber}

A semi-anechoic chamber was used for acoustic data collection because it provided the same FF test conditions in a controlled indoor environment. The chamber dimensions are shown in Figure 3.1. The shapes labeled 1 and 2 represent a vehicle loading door and a standard door respectively. The four walls and ceiling were covered in a sound absorbing foam minimizing reverberations from those surfaces. The floor was comprised of hard concrete with a smooth surface. These surfaces are displayed in Figure 3.2. Correction measures were applied to mitigate the chance of any reflections from the ground to the microphone receivers. This is further discussed in the subsequent section. 


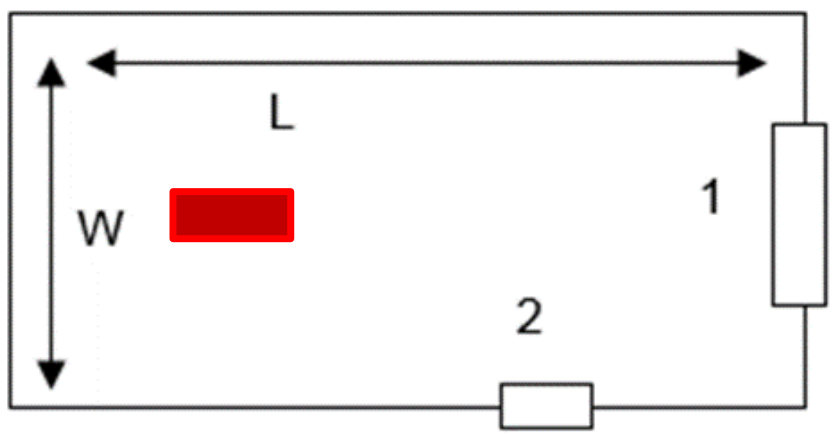

Figure 3.1: Simplified layout of the semi-anechoic

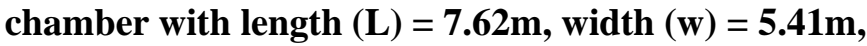
and height $(h)=2.8 \mathrm{~m}$. The red box indicates the location of the apparatus during testing.

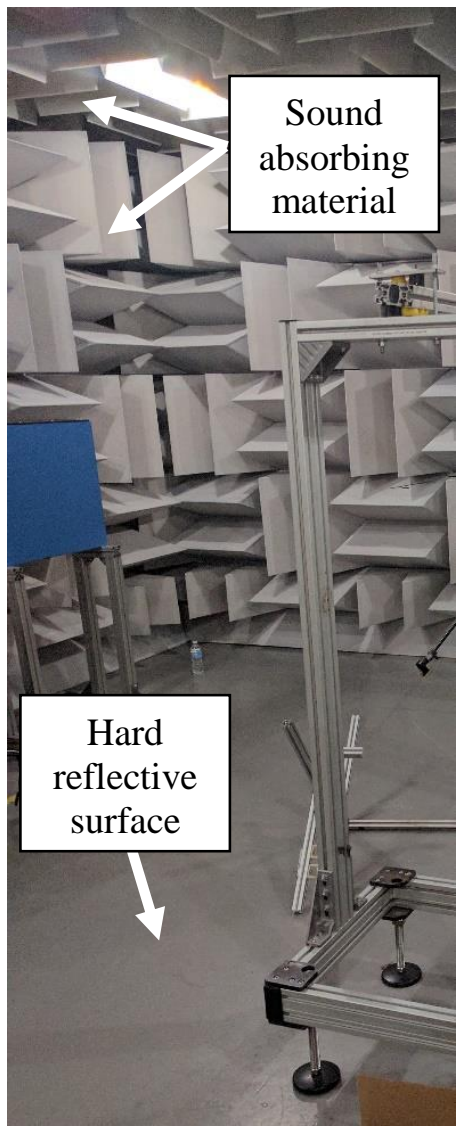

Figure 3.2: Semi-anechoic chamber lined with sound absorbing material. 


\subsubsection{Testing Apparatus}

This research focused on the acoustic phenomena of an automotive door closure independent of vehicle assembly. To simulate the kinematics of a door closing motion, the isolated latch and striker were affixed to a double vertical pendulum apparatus composed of aluminum alloy. The latch mechanism was fastened to one pendulum arm and the striker was attached to the other arm. The arms are allowed to swing towards each other causing the striker to engage with the latch mechanism. The double pendulum device ensured that the boundary conditions present in this apparatus were equivalent to the boundary conditions present for a striker and latch properly affixed to a vehicle assembly. The apparatus is shown in Figure 3.3. A vertical pendulum was used for this type of experiment over a horizontal swinging apparatus due to its simple and effective design. The pendulum operated using gravitational potential energy, eliminating the need for an external drive motor to facilitate the door closing motion. Moreover, the lack of external power allowed this device to operate without producing additional sounds, creating an environment where the only sound present was that of the latch during operation.

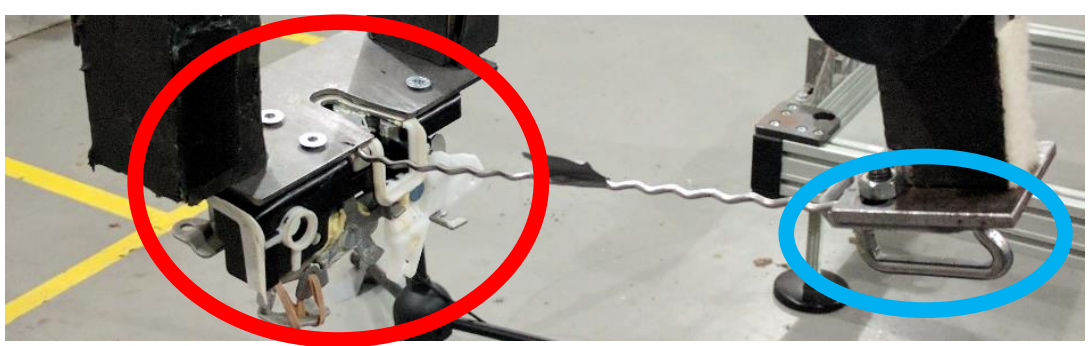

Figure 3.3: The latch mechanism is highlighted by the red circle and the striker is highlighted by the blue circle. 


\subsubsection{Microphone Array and Calibration}

The data collection process adhered to an internal corporate testing protocol Sound pressure measurements were collected using a three-element condenser microphone array. These elements were placed at three locations around the latch denoted by MP1, MP2 and MP3, each $0.5 \mathrm{~m}$ from the latch. The microphone array is depicted in Figure 3.4. Figure 3.5 demonstrates the position of the microphone receiver ends normal to the direction of the propagating sound waves. As per the testing protocol, LMS Test.Lab was used to calibrate the three microphones used for data collection. Table 3.1 depicts the calibration settings for each of the three microphones.

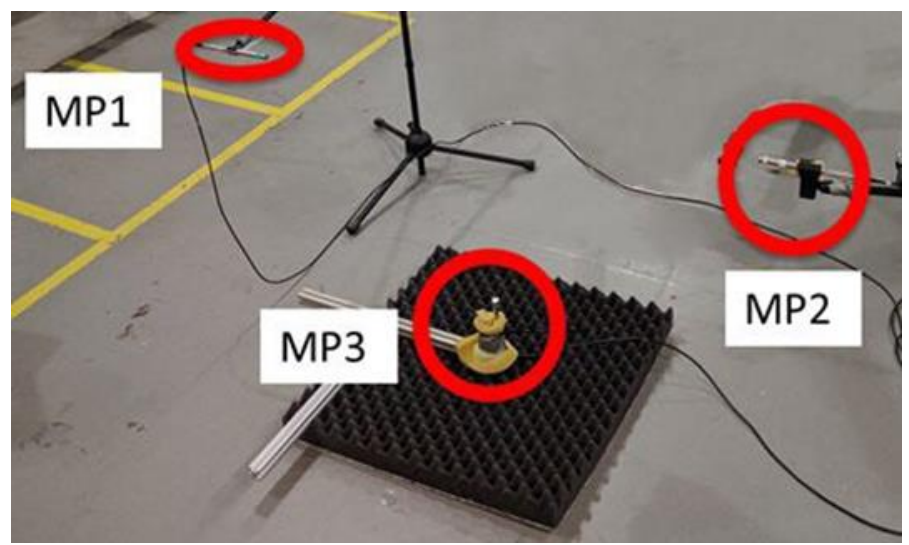

Figure 3.4: Three-element microphone array. 

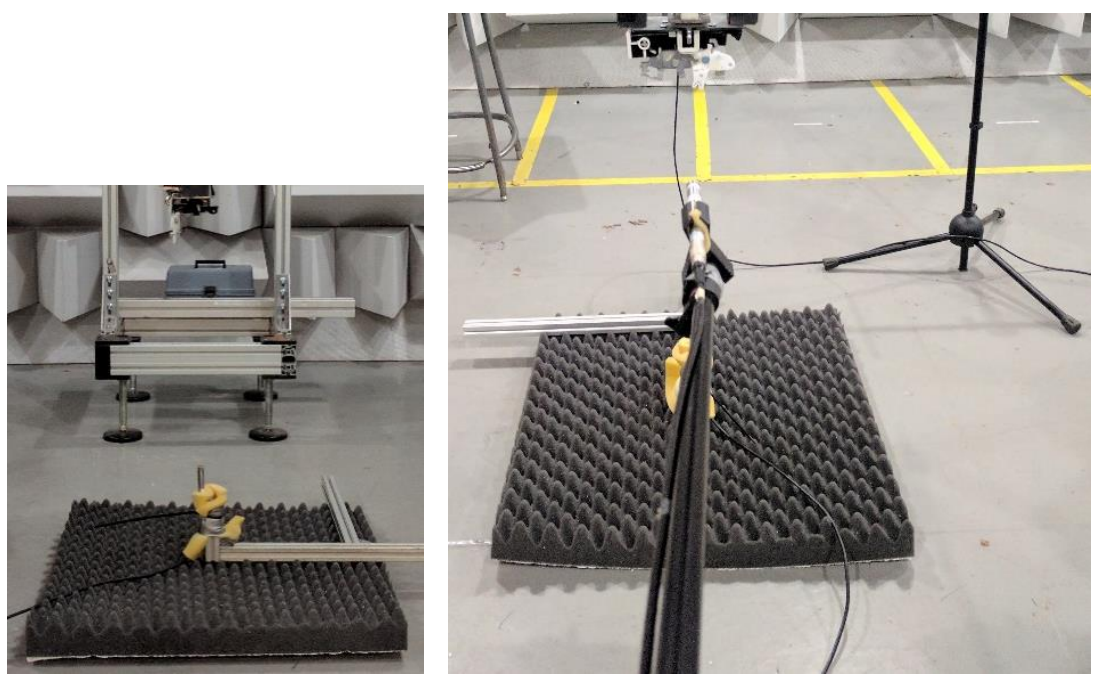

Figure 3.5: Side (left) and front (right) view of the microphones normal to the latch.

Table 3.1: Calibration settings for the microphone array.

\begin{tabular}{|c|c|}
\hline Microphone & $\begin{array}{c}\text { Sensitivity } \\
{[\mathbf{m V / P a}]}\end{array}$ \\
\hline MP1 & 51.63 \\
\hline MP2 & 45.71 \\
\hline MP3 & 46.81 \\
\hline
\end{tabular}

\subsubsection{Data Acquisition System}

The LMS Test.Lab Data Acquisition System (DAQ) recorded sound pressure measurements from the microphone array. The propagation of sound from source to receiver was governed by the Wave Equation given by Equation 3.1.

$$
\nabla^{2} p-\frac{1}{c^{2}} \frac{\partial^{2} p}{\partial t^{2}}=0
$$


where $\nabla^{2}$ is the Laplacian operator, $p$ is the local deviation from ambient pressure, and $c$ is the speed of sound in an acoustic media [11][30]. To prevent inaccurate sampling issues such as aliasing, the sampling frequency should be equivalent to the Nyquist Frequency dictated by Equation 3.2.

$$
f_{N}=2 f
$$

where $f_{N}$ is the Nyquist frequency and $\mathrm{f}$ is the highest frequency present in the recorded signal [6]. This results in a Nyquist frequency of $40 \mathrm{kHz}$. However, the collected data were sampled at a frequency of $81,920 \mathrm{~Hz}$ which is well above the Nyquist frequency.

\subsubsection{Entrance Speed Calculation}

In accordance with the testing protocol, a target speed of $0.8 \mathrm{~m} / \mathrm{s}$ (striker speed relative to the latch) was required for acoustic measurements. This is equivalent to the average closing speed necessary to achieve a fully latched position. An additional target speed of $1.2 \mathrm{~m} / \mathrm{s}$ was added to the testing procedure. This is equivalent to a more aggressive door slam. The addition of a faster closing speed was used to provide insight into how the impact speed of the striker relative to the latch would influence the overall perceived quality of the radiated sound. Due to the complex dynamics of the double pendulum apparatus, an oscilloscope speed trap allowed for the precise separation distance necessary to achieve each target speed.

The speed trap, shown in Figure 3.6, operated using laser motion detection. As an object with a known dimension passed through the laser, the time duration for the object to completely pass the laser was recorded. This allowed for the precise measurement of the striker entrance speed relative to the latch body. The relationship is given in Equation 3.3.

$$
V_{r e l}=\frac{w_{s t}}{t_{s t}}
$$


where $V_{r e l}$ is the relative speed, $w_{s t}$ is the width of the moving object and $t_{s t}$ is the time it takes the object to pass through the laser. The resulting entrance speeds used for this test were $0.851 \mathrm{~m} / \mathrm{s}$ and $1.179 \mathrm{~m} / \mathrm{s}$ with a percent different from the target speeds of $6.4 \%$ and $1.78 \%$ respectively. The percent differences were small enough to be considered negligible and it was deemed appropriate to move forward with the testing procedure. Metal rods were then cut to the exact separation distance necessary for the desired impact speed. This eliminated the possibility of entrance speed error for each test trial. Figure 3.7 depicts the metal rods used during the experiment.

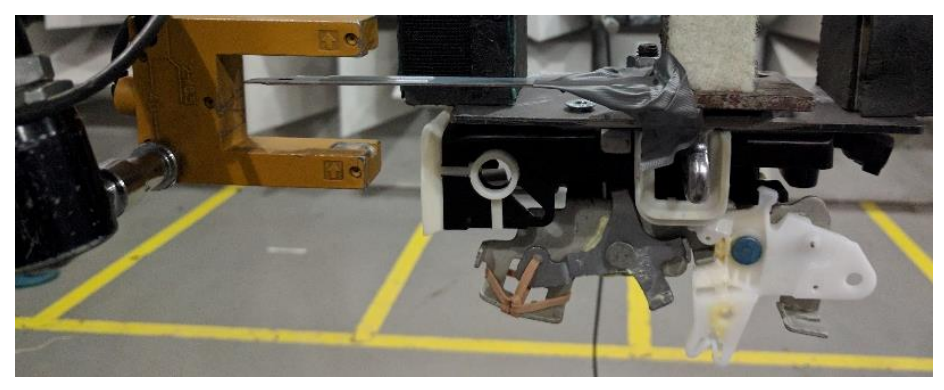

Figure 3.6: Speed trap device attached to the pendulum apparatus.

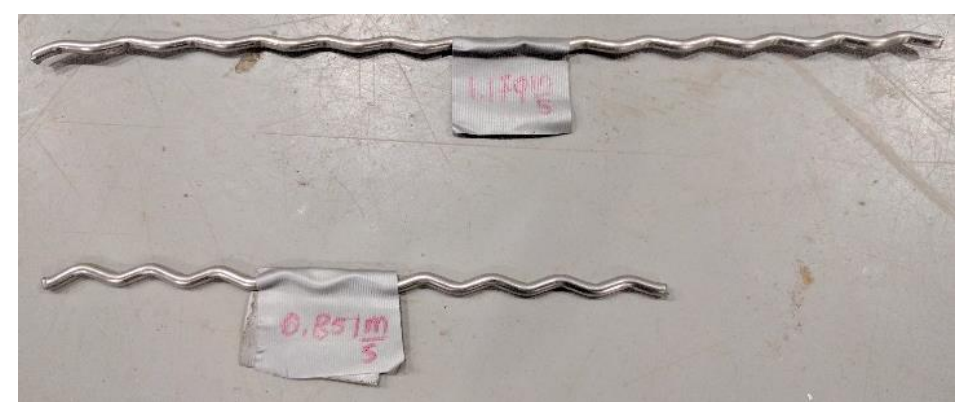

Figure 3.7: Metal rods used to achieve $1.179 \mathrm{~m} / \mathrm{s}$ (top) and $0.851 \mathrm{~m} / \mathrm{s}$ (bottom) entrance speeds. 


\subsubsection{Sound Field Boundary Conditions}

As previously mentioned, it is ideal to take acoustic sound pressure measurements from within the FF region of an anechoic or semi-anechoic chamber. Reverberations from the walls in the semi-anechoic chamber used were mitigated using the sound absorbing material seen in Figure 3.2. This resulted in a sound field that was comprised of three regions. The region immediately adjacent to the sound source is denoted as the Hydrodynamic Near Field (HNF). This region experiences fluid motion that is not directly linked to sound propagation and not ideal for acoustic measurements [30]. The Geometric Near Field (GNF) is the sound region directly adjacent to the HNF. This region experiences interference between contributing waves from different parts of the latch. Acoustic measurements of broadband sounds in the GNF are not always preferred and should be avoided if boundary conditions allow. The FF region directly adjacent to the HNF is considered the best region suited for data collection.

It is possible to establish the location of the FF given certain parameters of the environment as well as the sound source. For the purpose of this work, variables $\gamma$ and $\kappa$ were the parameters used to determine the location of the FF. These parameters are given in Equation 3.4 and Equation 3.5 respectively [30].

$$
\begin{gathered}
\gamma=\frac{2 r}{l} \\
\kappa=\frac{\pi l}{\lambda}
\end{gathered}
$$

where $\gamma$ is the first FF parameter, $r$ is the distance from the source to the measurement position, $l$ is the characteristic source dimension (typically the longest dimension on the vibrating structure), $\kappa$ is the second FF parameter and $\lambda$ is the largest wavelength present in the radiated sound. The input parameters applied to Equation 3.4 and Equation 3.5 were: $r=0.5 m, l=0.1684 m, \lambda=160 \mathrm{~Hz}$ resulting in a localized position within the transition region given by the red marker in Figure 3.8. This is the boundary between the GNF and the FF. Due to the inherent limitations of the testing procedure, receiver locations, 
and the dimensions of the latch, the location of the microphone array within the transition region was deemed acceptable.

This plot indicates the data collected at $160 \mathrm{~Hz}$ and above is accurate, however any results found at frequencies below $160 \mathrm{~Hz}$ are found within the HNF and were omitted for this work. Therefore, the lower bound for this analysis was found to be $160 \mathrm{~Hz}$ with an upper bound of $5 \mathrm{kHz}$ as per the limitation set out at the beginning of this work.

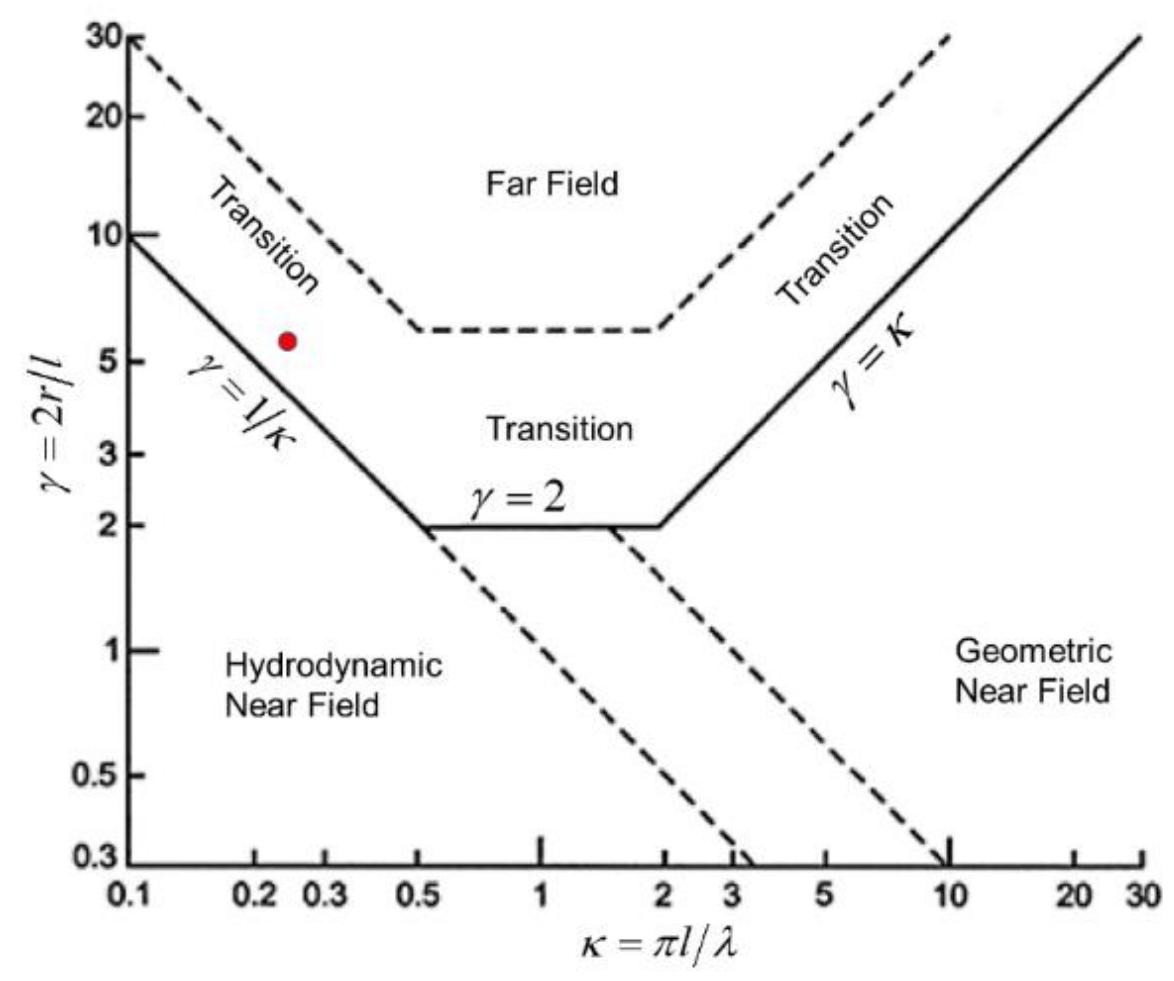

Figure 3.8: Depiction of the radiating sound field [30].

As previously indicated, the hard concrete floor allowed for sound to be reflected back into the room interior. This would result in sound contributions from direct sound pressure as well as reverberated sound pressure which is not desired. An acoustic absorber was placed directly below the double pendulum apparatus as outlined in Figure 3.4. The absorber was made of polyurethane foam with an egg crate texture on the face parallel to the floor surface and closest to the apparatus. The dimensions were 
$0.6604 \mathrm{~m}$ long by $0.6604 \mathrm{~m}$ wide by $0.0762 \mathrm{~m}$ thick. A dimension of $0.0762 \mathrm{~m}$ allowed for $95 \%$ of the direct sound pressure to be absorbed [32]. The placement of the foam absorber directly below MP3 ensured that the absorber did not obstruct the sound path from the source to the receiver.

Geometrical acoustics defines sound travelling in straight lines propagating outward from the sound source in the form of sound rays [6]. Figure 3.9 shows the propagating sound rays in the vicinity of the latch that could be reflected back to MP1 and MP2. The latch and striker are represented by the red box affixed to the pendulum shown in white. MP1, MP2 and MP3 are represented by the three grey rectangles and the sound absorbing foam is shown as a black hatched box. The Law of Reflection reveals the propagating sound ray (incident ray) intersecting with the reflected ray along the normal line shown as a hashed line [6]. The normal line was determined to be $0.25 \mathrm{~m}$ from the latch. The graphic confirms the chance of any reflected sound rays measured by MP1 and MP2 is greatly reduced as the absorbing foam extends beyond the normal line.

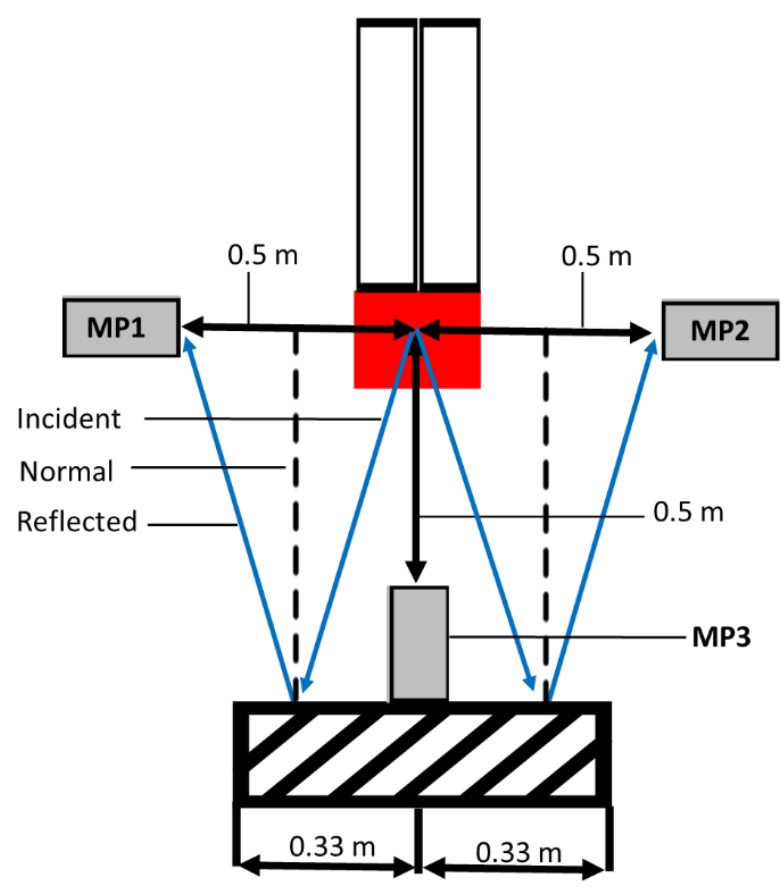

Figure 3.9: Incident and reflected sound rays. 


\subsection{Experimental Procedure}

Experimental testing involved measuring the radiated sound pressure in pascals $(\mathrm{Pa})$ at three different microphone locations during latch operation. Before data collection began, the ambient sound pressure level of the room was assessed. Three trials were performed, resulting in an overall sound pressure level that was below 30dB, which is equivalent to a "very quiet" room. Appendix A displays a standard decibel (dB) scale used in acoustic research. With this result, the testing environment was considered acceptable for acoustic testing.

A total of five latches (Figure 3.10) were analyzed at two different striker entrance speeds of $0.851 \mathrm{~m} / \mathrm{s}$ and $1.179 \mathrm{~m} / \mathrm{s}$. The latch specimens were fabricated and greased to specification the day of testing. The test specimen was placed in the pendulum apparatus via three steel fasteners. The same striker was used for each trial. Before data was collected, one metal rod was situated between metallic mounting points on each pendulum arm. These points, seen in Figure 3.11, allowed for swift removal of the rod.

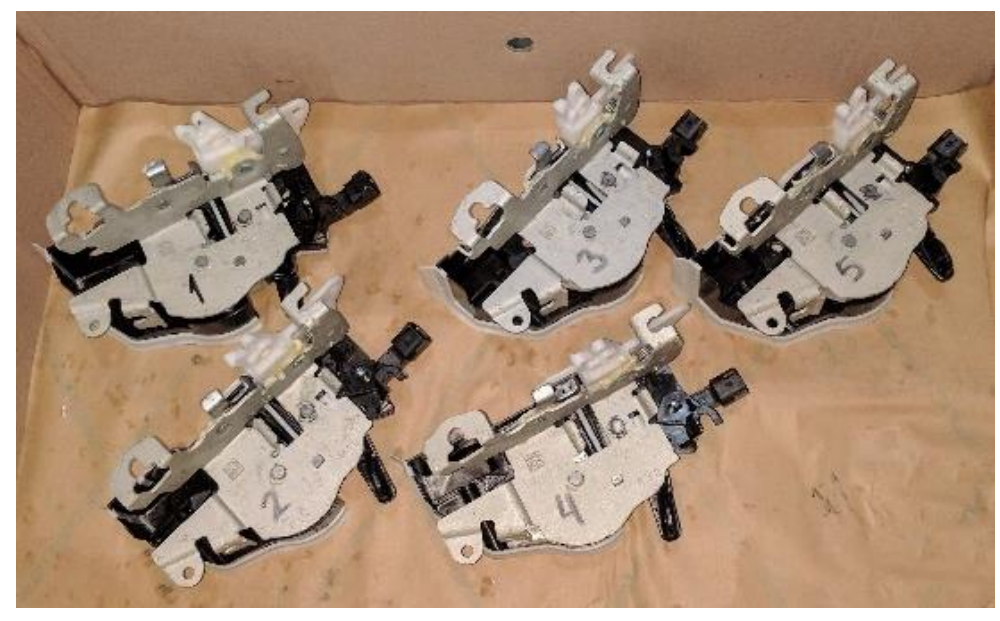

Figure 3.10: Latches labeled 1-5 used in the investigation.

Prior to the commencement of the trial, no sounds were made within the chamber. The DAQ began recording the sound pressure using the microphone array. An experimenter would then swiftly remove the rod causing the pendulum arms to swing toward each other, engaging the striker with the mechanism. It 
should be mentioned that the swift removal of the rod caused some sound that was picked up by the three receivers. The effect of this unwanted noise was removed during the post-processing procedure and was not included in any sound pressure calculations. After the sound decayed, the DAQ would cease recording. This procedure from start to end was identified as one test trial.
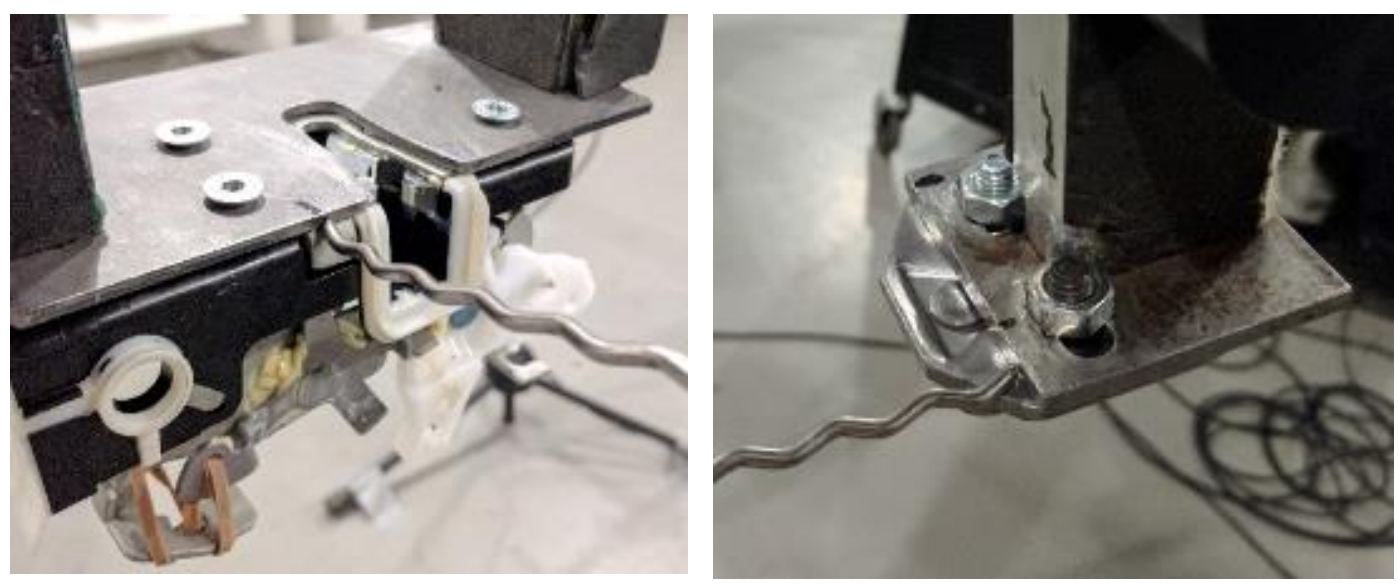

Figure 3.11: Placement of the metal rods.

For each of the five latches and two impact speeds, three trials were performed for a total of thirty test trials containing ninety test samples. Each sample recording lasted approximately ten seconds to ensure the total acoustic response from excitation to decay was captured. The raw microphone data was postprocessed in MATLAB to identify distinguishing features of the acoustic phenomena.

\subsection{Experimental Post-Processing Procedure and Methodologies}

\subsubsection{MATLAB Truncation Algorithm}

A MATLAB script was created to automatically truncate the portion of the sound pressure containing the latch locking operation waveform from the raw data. The MATLAB script can be found in Appendix B with detailed comments. Figure 3.12 and Figure 3.13 display the raw un-processed recorded waveform before and after the truncation algorithm was applied. Figure 3.14 and Figure 3.15 exhibit the same truncation procedure for the $1.179 \mathrm{~m} / \mathrm{s}$ speed. The beginning of each truncated waveform identified the moment immediately prior to striker and fork-bolt contact. Similarly, the end of the waveform identified 
the moment immediately after the acoustic decay of the generated sound. MATLAB recorded the magnitude and position of the peak sound pressure. For each sample, the peak sound pressure was always caused by the latch locking operation. Due to the large quantity of data samples, only a subset of plots relating to Latch 3 will be included in this section. Throughout this work, MP1, MP2 and MP3 are represented by the blue, red and gold coloured data points respectively.

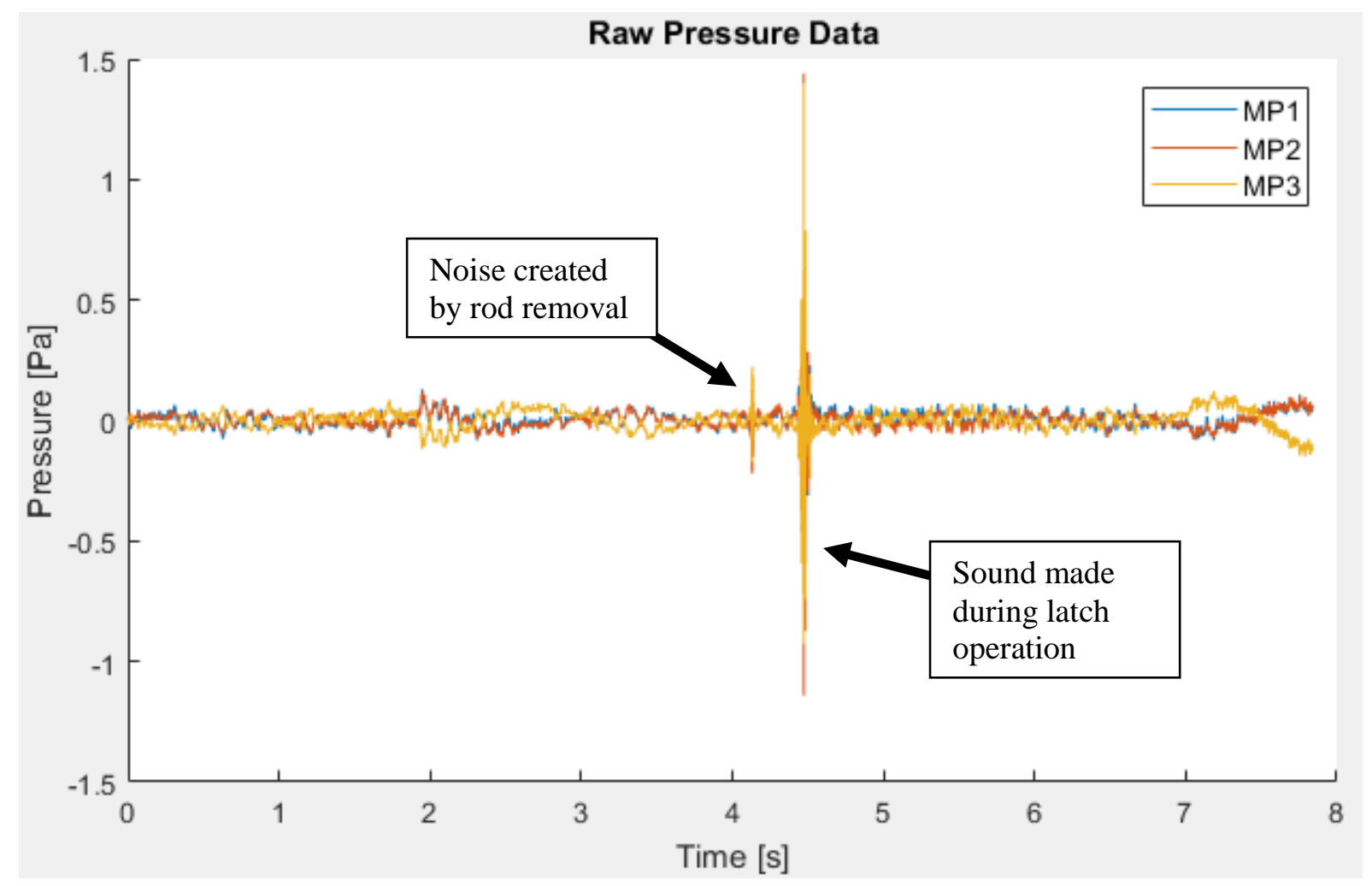

Figure 3.12: Entire sample of the recorded sound pressure for one trial at $0.851 \mathrm{~m} / \mathrm{s}$. 


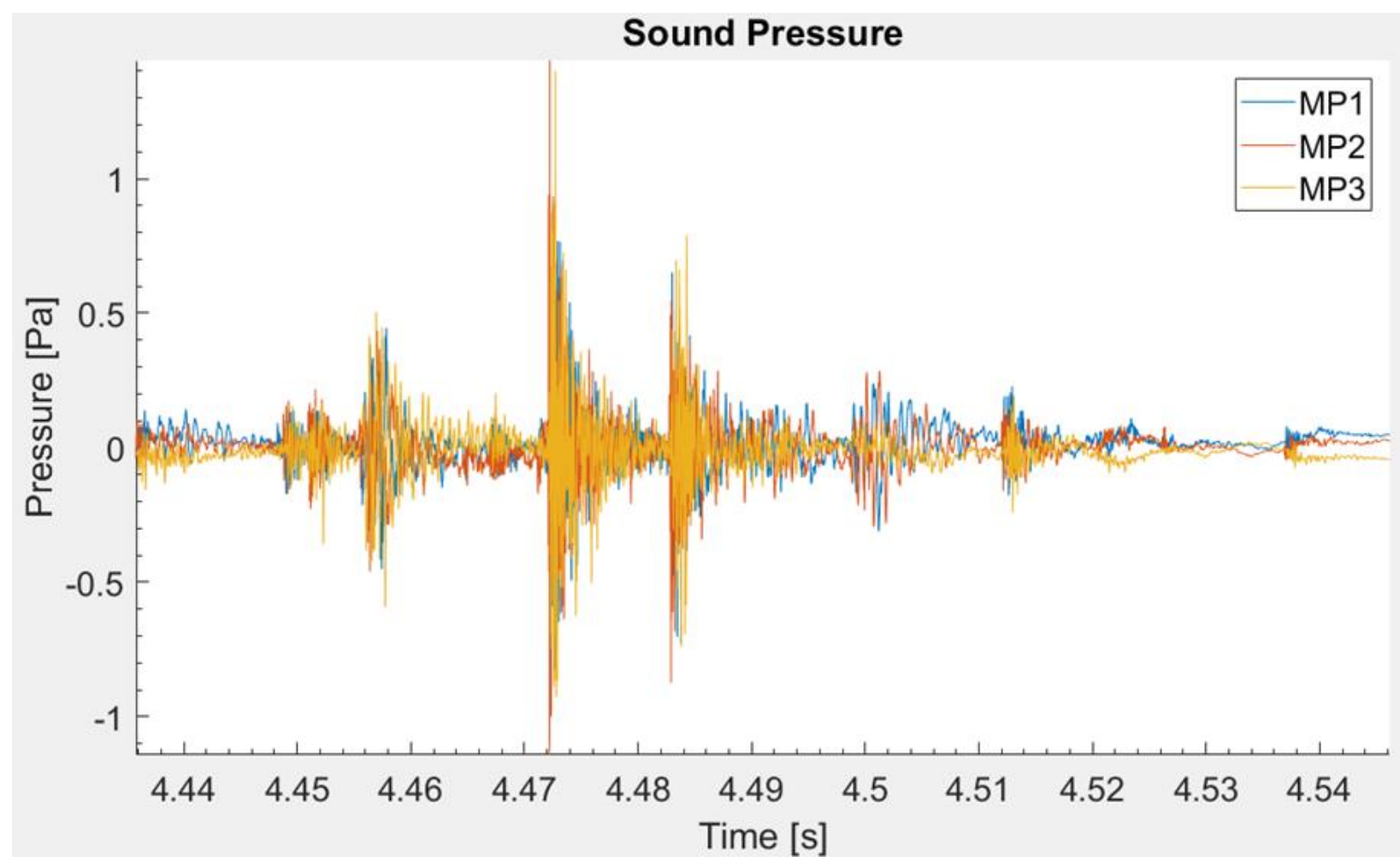

Figure 3.13: Extracted latch operation sound pressure waveform for one trial at $0.851 \mathrm{~m} / \mathrm{s}$.

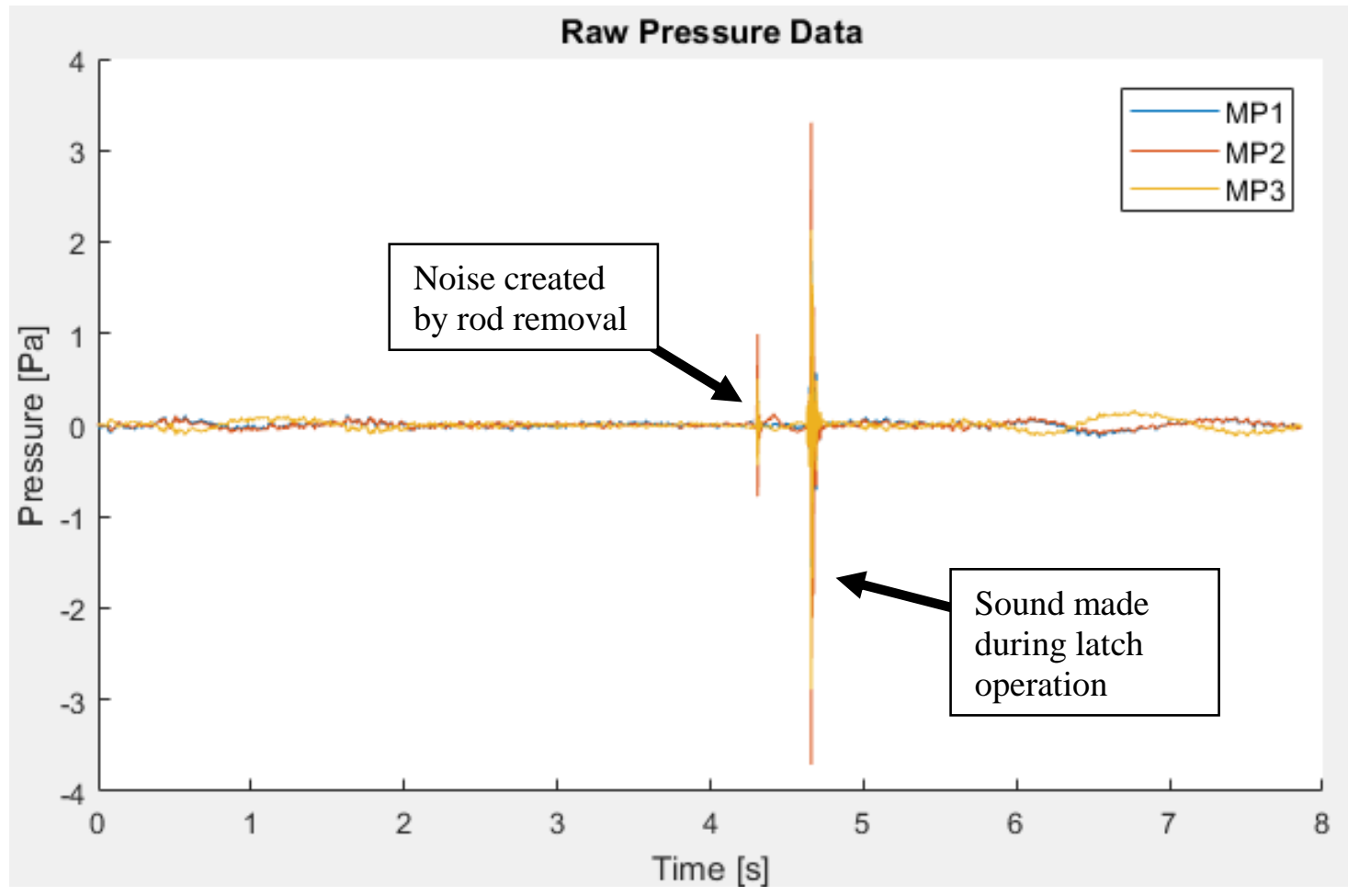

Figure 3.14: Entire sample of the recorded sound pressure for one trial at 1.179m/s. 


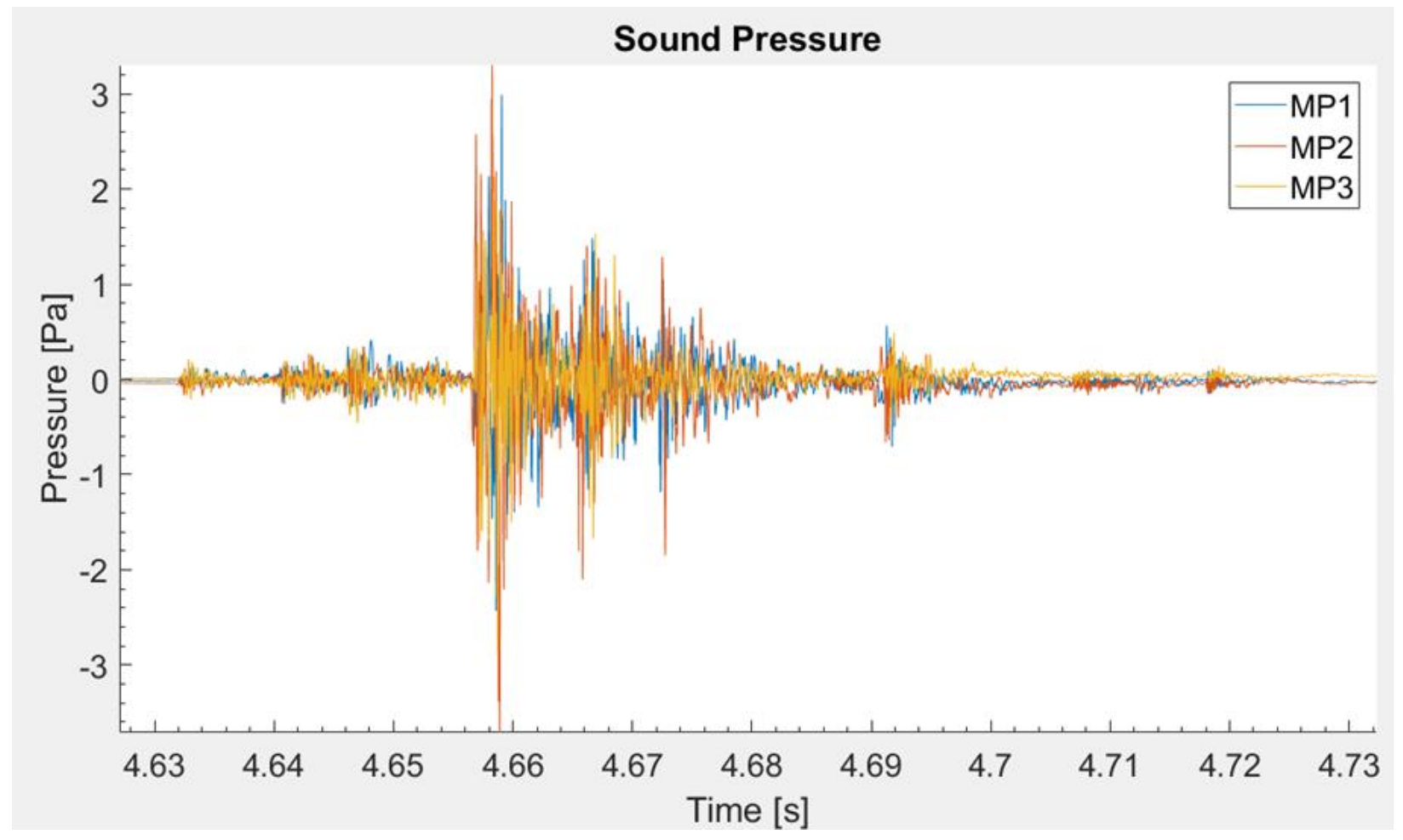

Figure 3.15: Extracted latch operation sound pressure waveform for one trial at $1.179 \mathrm{~m} / \mathrm{s}$.

In order to study the frequency composition of the acoustic response, two investigations were performed; a continuous wavelet transformation (CWT) analysis and a comparison of the average sound pressure level relative to a $1 / 3$ octave bandwidth.

\subsubsection{1/3 Octave Bandwidth and A-Weighting Filters}

Following the work performed by Von Estorf et al. [28], an analysis to determine the SPL magnitude at specific 1/3 octave bands was conducted. To achieve this, 1/3 octave band and A-weighting filters were required. For the purpose of this research a publicly available MATLAB 1/3 octave band filter bank script created by Dr. Christophe Couvreur was used [33].

It has been reported that there is a subset of frequencies within the band of human hearing that the ear is more sensitive to. This trend was observed in a study by Fletcher and Munson in 1933 where they investigated the loudness of pure tones in the audible spectrum relative to a pure $1 \mathrm{kHz}$ sound (reference 
tone) [34]. This phenomenon is represented by the Equal Loudness Contour (ELC) plot shown in Figure 3.16 [34]. The curves "indicate the sound pressure level required at any frequency in order to give the same apparent loudness as a $1 \mathrm{kHz}$ tone" [7]. The unit associated with the loudness of a sound is phons. Their study found that the human ear is most sensitive at hearing tones between $2 \mathrm{kHz}-5 \mathrm{kHz}$ due to the resonances that occur in the external acoustic meatus (ear canal) [7] [18] [35].

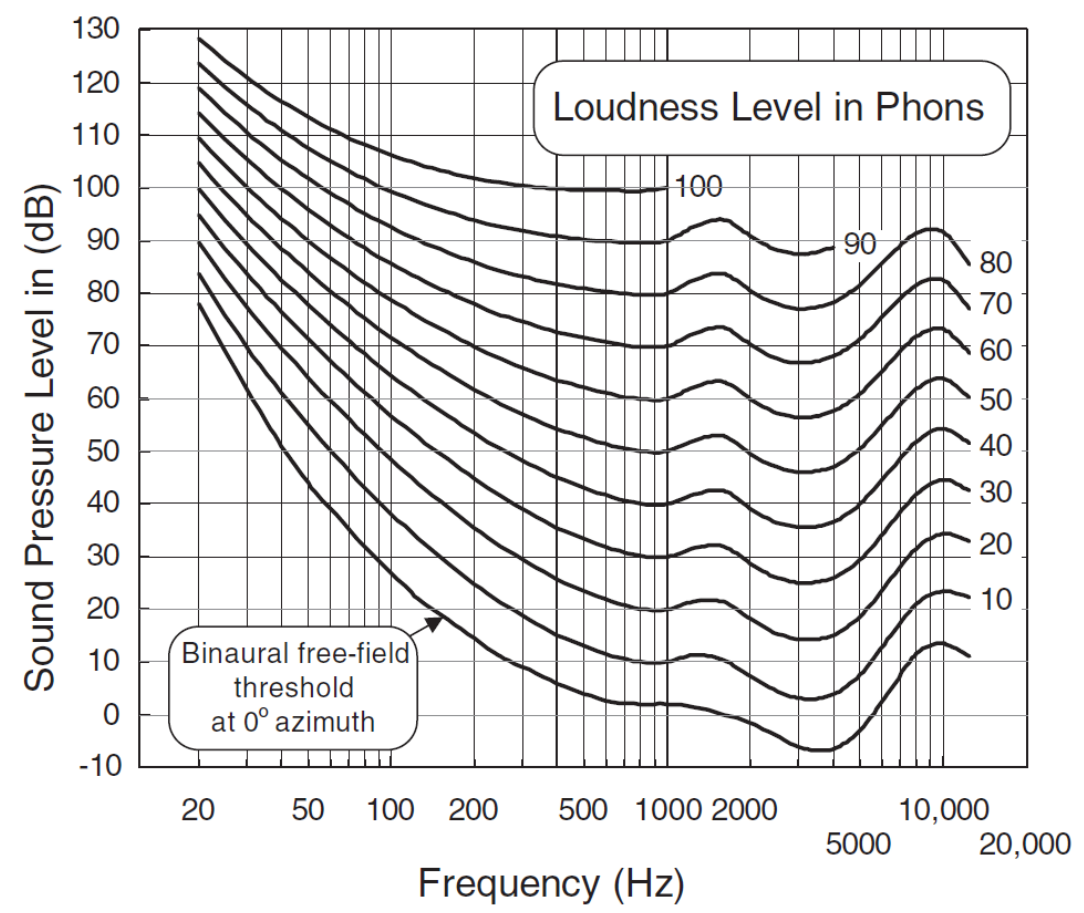

Figure 3.16: Revised Equal Loudness Contour plot stated in ISO 226 [35]. The binaural threshold is denoted as the lowest level of sound a human can hear.

With the understanding that humans are more sensitive to some frequencies and not others, it is necessary to apply weighing functions to the recorded data to compensate for this sensitivity variation. The two most common internationally standardized weighing networks are the " $\mathrm{A}$ " and " $\mathrm{C}$ " networks [8]. Figure 3.17 depicts the frequency weighing networks in a standard graphical form. According to Goelzer et al. (2001), "These networks 'weight' the contributions of the different frequencies to the over-all sound level, so that sound pressure levels are reduced or increased as a function of frequency..."[8]. The "A" 
and "C" functions modify the recorded waveform to follow the equal loudness curves at 40 phons and 100 phons respectively as shown in Figure 3.16. The A-weighting network is the most common network used in industry. In keeping with industry protocol, this network was adopted for this research. SPL results with an A-weighting network applied are identified with units dBA.

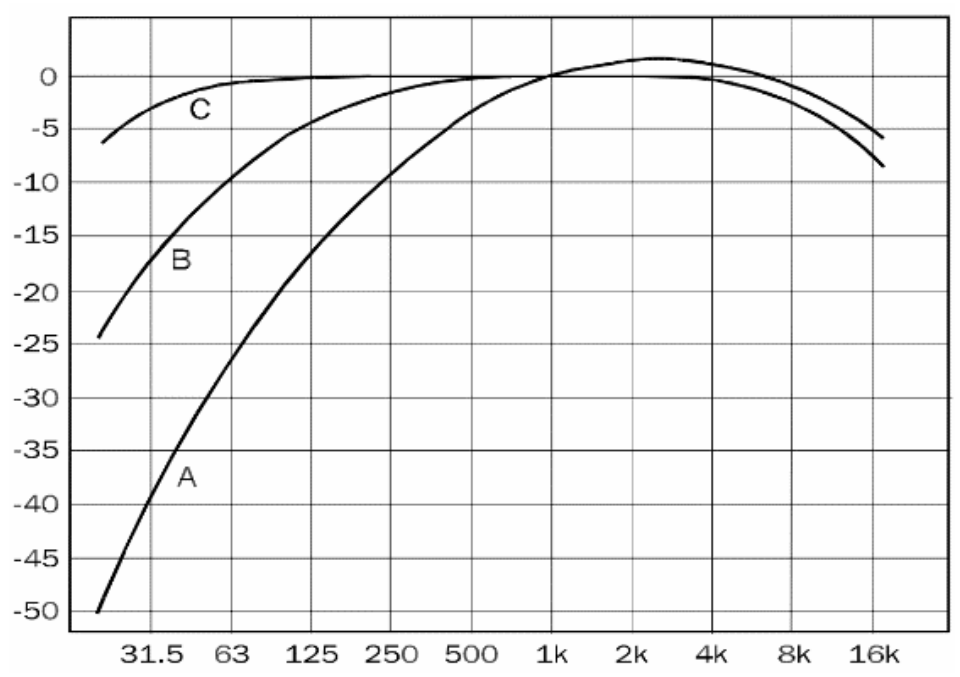

Figure 3.17: "A", "B" and "C" weighting networks. Gain [dB] represented by the $y$-axis and frequency $[\mathrm{Hz}]$ on the $\mathrm{x}$-axis [8].

A representation of the actual A-weighting network used to attenuate and amplify the frequency content of the D21MC latch is represented in Figure 3.18. The blue curve represents the weighting function, the dashed lines represent the upper and lower bounds of a Class 1 filter and the dotted lines represent that of a Class 2 filter. According to the International Electrotechnical Commission (IEC) 61672-1:2013 international standard, Class 1 and Class 2 are identified as acoustic filters with a specified tolerance mask. A Class 1 filter represents a mask with strict tolerance specifications, whereas a Class 2 filter represents a mask with less strict tolerance specifications. A Class 1 filter was used for this experiment [36]. 


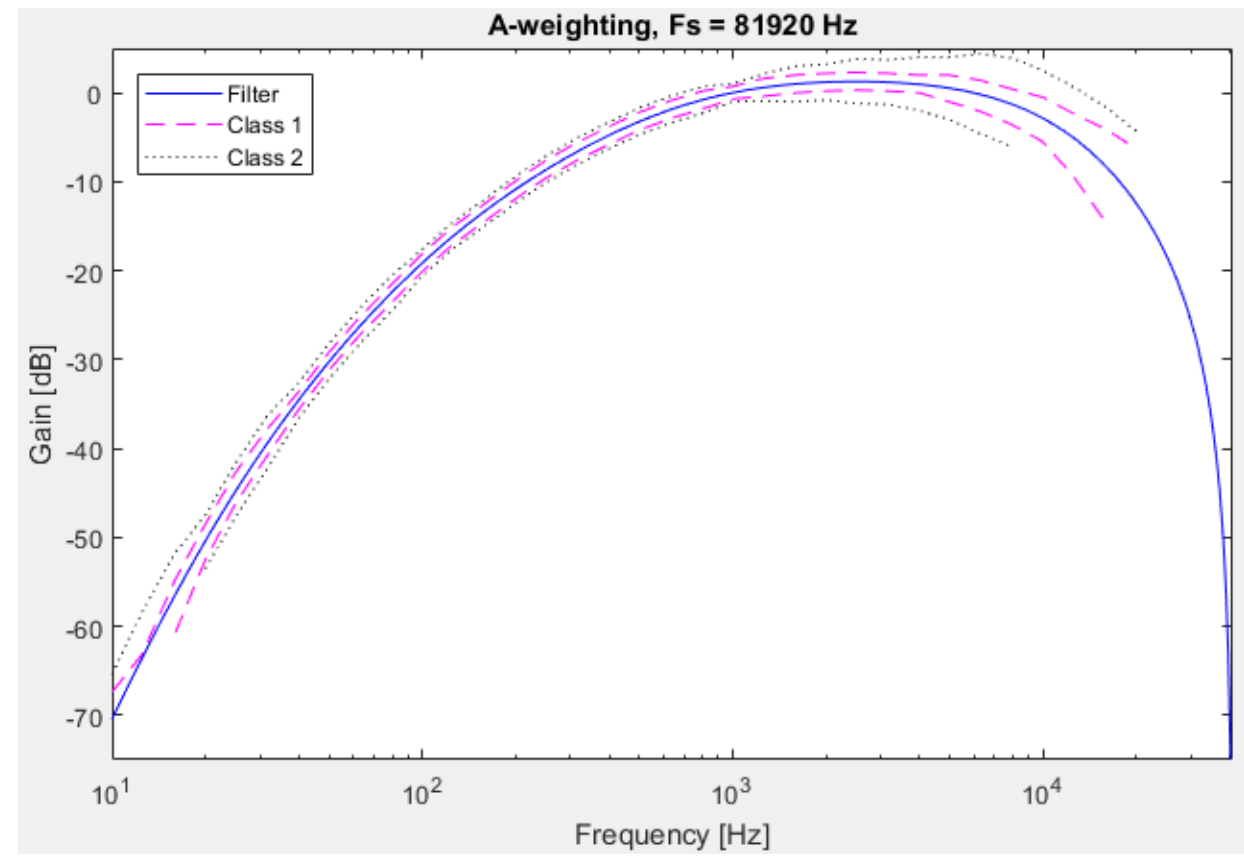

Figure 3.18: A-weighting network applied to D21MC data.

After the application of the A-weighing filter network, 1/3 octave band filters were applied to the sample to determine the frequency contribution. These filters conformed to filter specifications outlined in the IEC 61260-1 international standard [37]. Figure 3.19 is a sample of a 1/3 octave filter with a center frequency of $1 \mathrm{kHz}$. These filters allowed the sound to be decomposed into its frequency components and divided into sets of frequency bands. Each band encompassed a frequency range spanning one-third of an octave. The contribution of the frequencies in each band combined to an overall contribution for the $1 / 3$ octave denoted by its center frequency. The larger the contribution from the individual frequencies the greater the overall magnitude of the frequency band. 


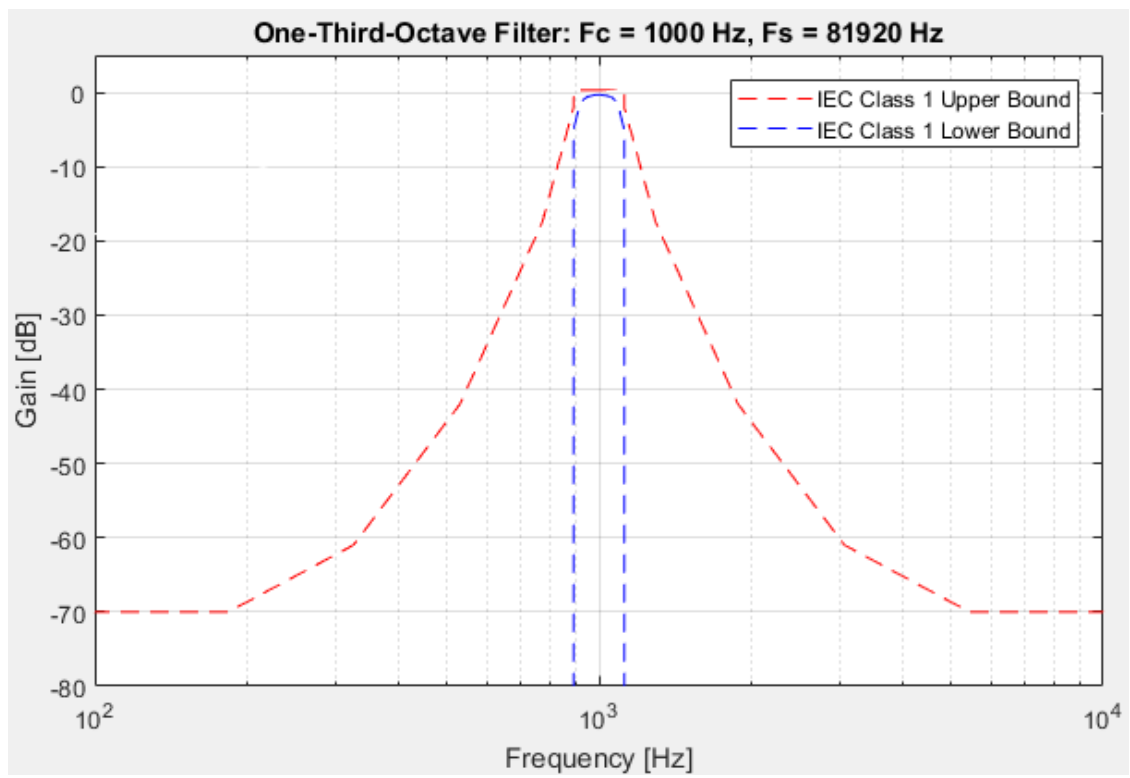

Figure 3.19: Class 1 1/3 octave filter with center frequency $1 \mathrm{kHz}$ applied to the D21MC data.

A filter bank, which is a collection of $1 / 3$ octave filters, allows acoustic researchers to display the contributions of specific frequency bands within the entire audible spectrum. For this study, filter banks were applied to the sound pressure data collected by each microphone. The results are presented in a sound pressure level versus 1/3 octave bandwidth plot shown in Figure 3.20. It should be noted that a negative SPL signifies a response that is below the level of human hearing. For clarity, this plot represents the SPL data of three microphone receivers of one test trial for Latch 3 at one striker entrance speed. Thirty plots were generated in total.

From this data, the addition of incoherent sound pressure levels was performed to obtain the average SPL for each latch. This relationship is given by Equation 3.6.

$$
S P L_{\text {avg }}=10 \log \left(\frac{1}{3}\left(10^{\frac{S P L_{1}}{10}}+10^{\frac{S P L_{2}}{10}}+10^{\frac{S P L_{3}}{10}}\right)\right)
$$


where $S P L_{\text {avg }}$ is the average sound pressure level and $S P L_{[]}$is a general incoherent sound pressure level.

The MATLAB script created for this procedure is included in Appendix C. The addition was performed in steps resulting in five plots depicting the average sound pressure level as a function of the $1 / 3$ octave band spectrum for each of the five latches. These plots are discussed in a subsequent section.

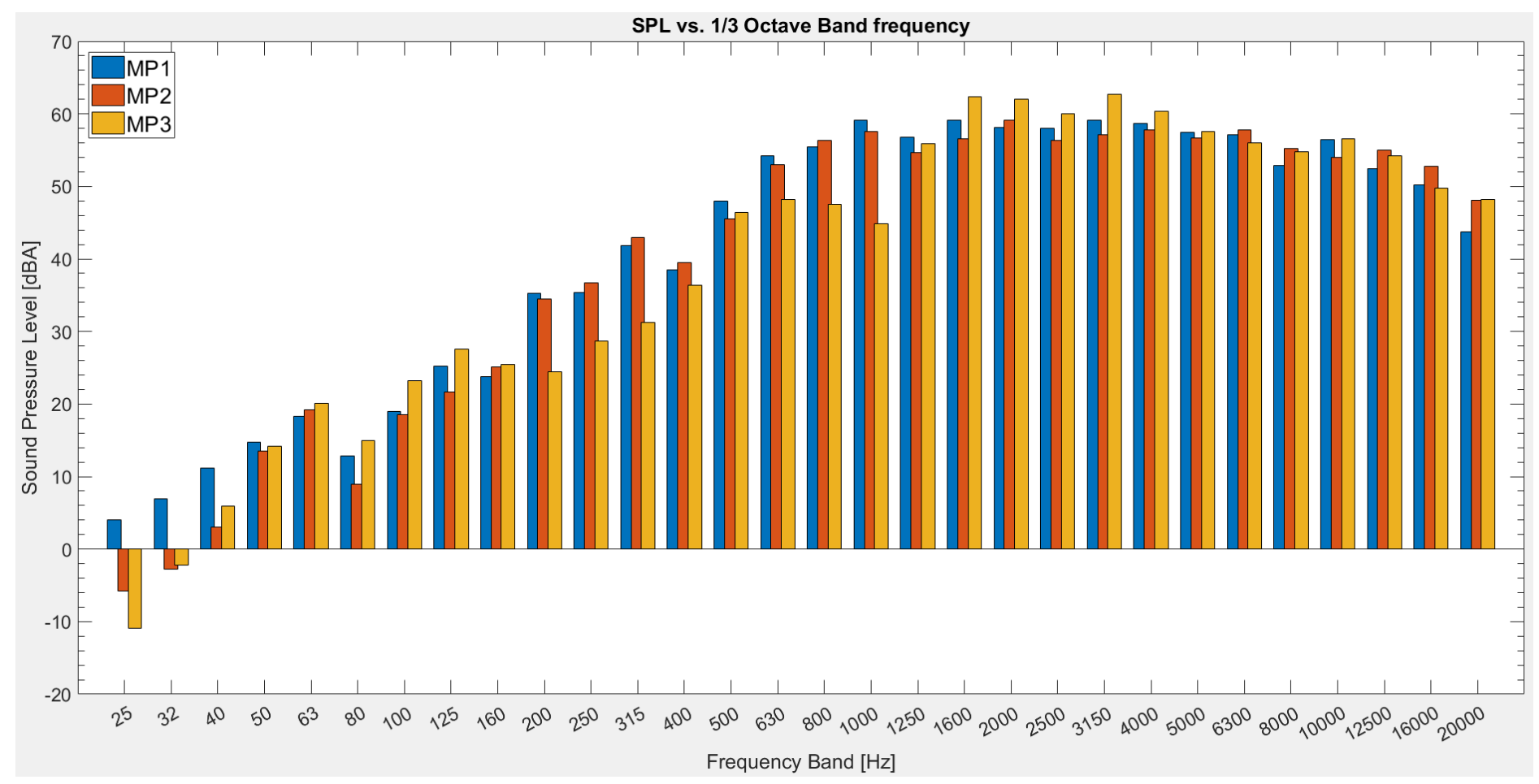

Figure 3.20: SPL versus 1/3 octave bandwidth for Latch 3 at $0.851 \mathrm{~m} / \mathrm{s}$ entrance speed.

\subsubsection{Continuous Wavelet Transformation Analysis}

A CWT is a modified Fourier transform optimized for analyzing abrupt changes in recorded data efficiently and accurately. The use of a CWT over a traditional STFT was discussed in Chapter 2. The recorded sample is analyzed using a finite wave-like oscillation which can be shifted and scaled [38]. The purpose of continuous wavelet signal analysis is to overcome the apparent lack of information in the frequency spectrum within a time-domain analog signal [39]. This relationship is given by Equation 3.7. 


$$
C(s, \tau)=\frac{1}{\sqrt{|s|}} \int_{-\infty}^{\infty} x(t) \psi\left(\frac{\tau-t}{s}\right) d t
$$

where $s$ is the window scaling parameter, $\tau$ is the window shifting parameter, $\psi((\tau-t) / s)$ is the "mother" wavelet, and $x(t)$ is the time signal. Morse wavelets are a family of complex valued analytic wavelets which are extremely useful for analyzing modulated signals [40]. They were chosen for their reliability and accuracy in signal processing [15].

\subsection{Results and Analysis}

The first set of analyses examined the transient waveform of each latch at each impact speed. Due to the large amount of data collected, only a subset of plots relating to Latch 3 is discussed. The remaining samples are included in Appendix D.

The experimental results for the impact speeds of $0.851 \mathrm{~m} / \mathrm{s}$ and $1.179 \mathrm{~m} / \mathrm{s}$ measured at each microphone are shown in Figure 3.21 and Figure 3.22 respectively. Time is represented on the $\mathrm{x}$-axis and pressure is represented on the y-axis. Initial inspection of the recorded waveforms showed good agreement between the sound pressures measured at each receiver with little variation. At $0.851 \mathrm{~m} / \mathrm{s}$ the peak sound pressure occurred at the middle of the latch locking operation with a magnitude slightly larger than 1.4Pa. As expected, peak sound pressure for the $1.179 \mathrm{~m} / \mathrm{s}$ impact speed was larger with a magnitude close to $3 \mathrm{~Pa}$. Analysis of each transient result proved that the sounds can be divided into 3 distinct segments. Segment 1 revealed that both speeds peak at a pressure of approximately $0.5 \mathrm{~Pa}$, indicative of the sounds generated by the striker sliding across the face of the fork-bolt in addition to the weak pawl and fork-bolt (ratchet) impact. 


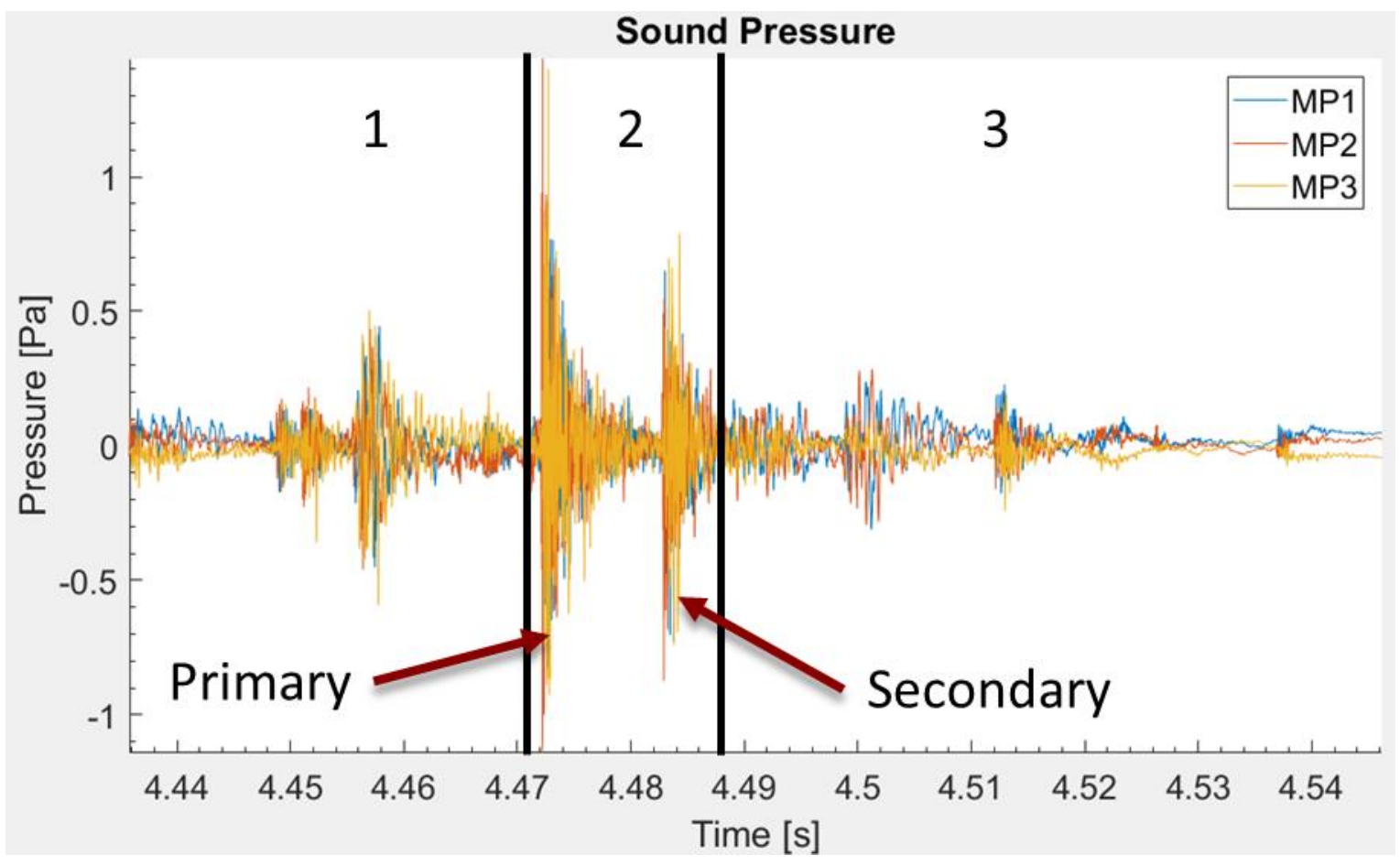

Figure 3.21: Segmented waveform of the $0.851 \mathrm{~m} / \mathrm{s}$ closure speed.

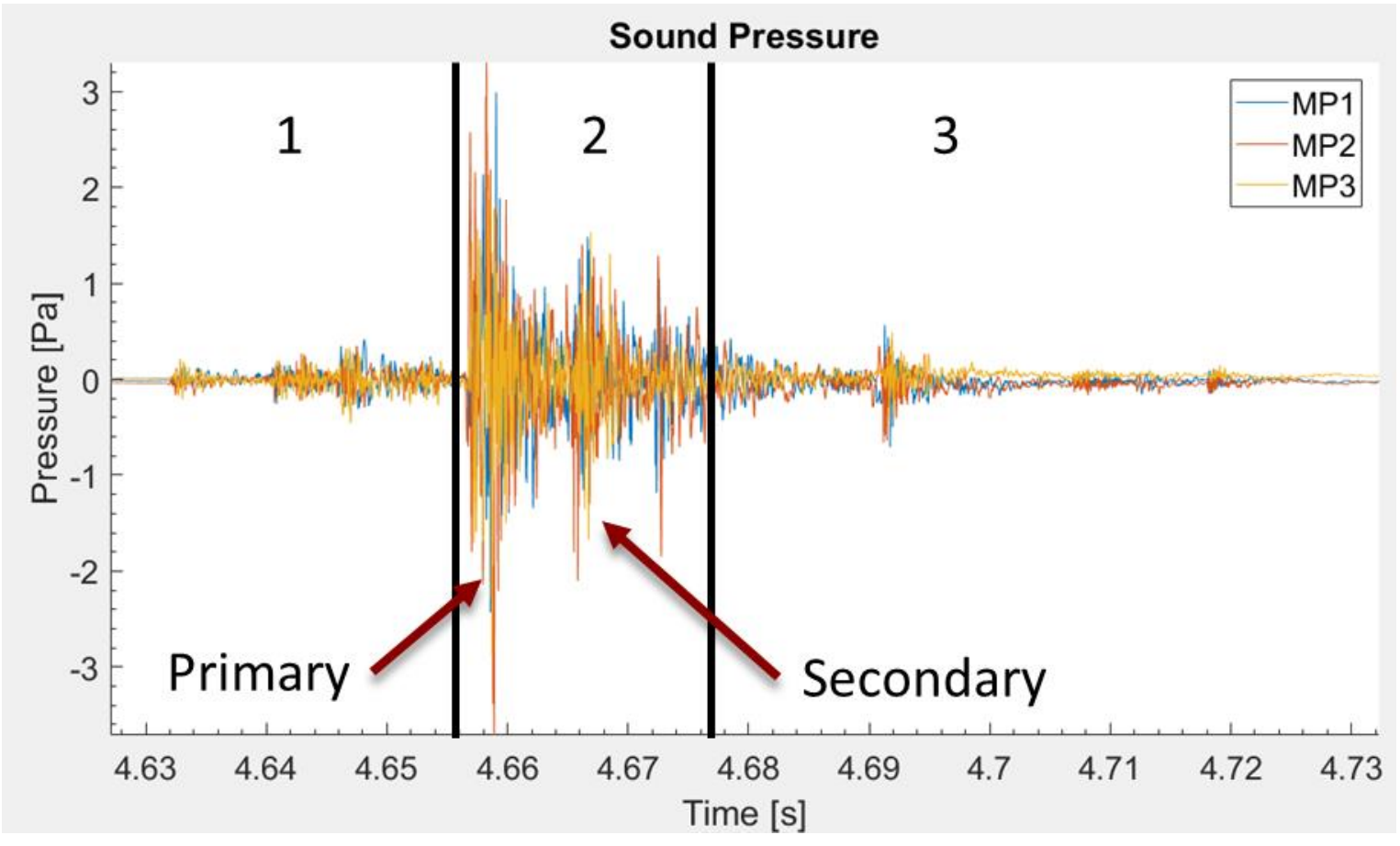

Figure 3.22: Segmented waveform of the $1.179 \mathrm{~m} / \mathrm{s}$ closure speed. 
In Segment 2, it is clear that two impact events (primary and secondary) govern the waveform. It was hypothesized that the dominant frequencies composing the sound were derived from these impact events. Additionally, it was theorized that the sound pressure was created by the striker/over-slam bumper impact as well as the ratchet/housing impact. Further analysis into the mechanics of the latch components is explained in more detail in a subsequent section.

An inherent difference between the sound pressures resulting from the different impact speeds is the distinguishability between the primary and secondary impact events. For both impact speeds, the time between the primary and secondary event are roughly $0.01 \mathrm{~s}$. This indicates that the impact events occurring within the latch are not necessarily dependent on the entrance speed of the striker.

A faster closing speed introduced additional kinetic energy into the system. The waveform shown in Figure 3.22 depicts the additional energy causing the sound pressure from the primary impact to overlap with the sound pressure generated by the secondary impact. This "blending" of sound pressure reduces the definition of the sound, giving the impression of a smeared acoustic response [10]. Segment 3 is a result of additional latch component interactions and residual vibrations which were significantly lower than the sound pressures generated within Segment 2. It can be seen in Appendix D that this trend is apparent between each recorded $0.851 \mathrm{~m} / \mathrm{s}$ sound sample and the $1.179 \mathrm{~m} / \mathrm{s}$ counterparts.

The average duration of the entire latch operation for all five latches was approximately $0.1073 \mathrm{~s}$ at $0.851 \mathrm{~m} / \mathrm{s}$ and $0.1054 \mathrm{~s}$ at $1.179 \mathrm{~m} / \mathrm{s}$. With a difference in average duration in the order of microseconds, it was considered to be negligible. As a result, there was no measurable difference to the duration of the latch locking operation due to an increase in relative speed of the striker entering the latch. 


\subsubsection{Continuous Wavelet Transform Analysis}

The CWT analysis was computed to determine the frequency composition of the waveform as a function of time. Figures 3.23-3.25 depict the wavelet analysis of the waveform shown in Figure 3.21. Normalized magnitude is represented by the colour bar, time is represented on the x-axis, and frequency is represented on the y-axis. For the purpose of this work only the frequencies below the red line were investigated. Justification for this limit is described in Chapter 1. The red circles outline the frequency with the largest magnitude produced by the latch. By comparing the timelines of Figure 3.21 and Figures 3.23-3.25, it is confirmed that the primary and secondary impact events dominate the acoustic response. Table 3.2 displays the dominate frequency ranges for each $0.851 \mathrm{~m} / \mathrm{s}$ plot.

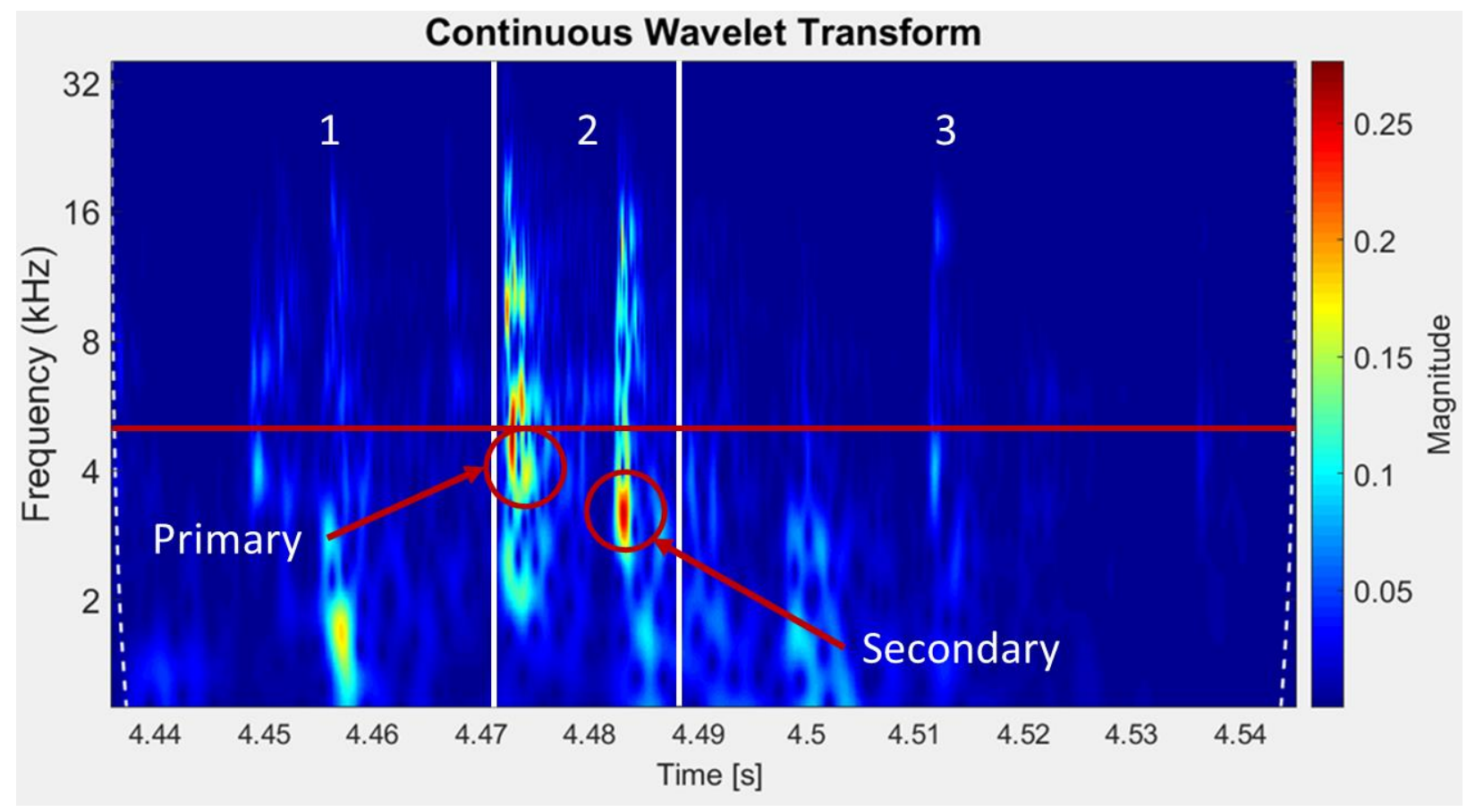

Figure 3.23: CWT of the $0.851 \mathrm{~m} / \mathrm{s}$ closure speed measured at MP1. 


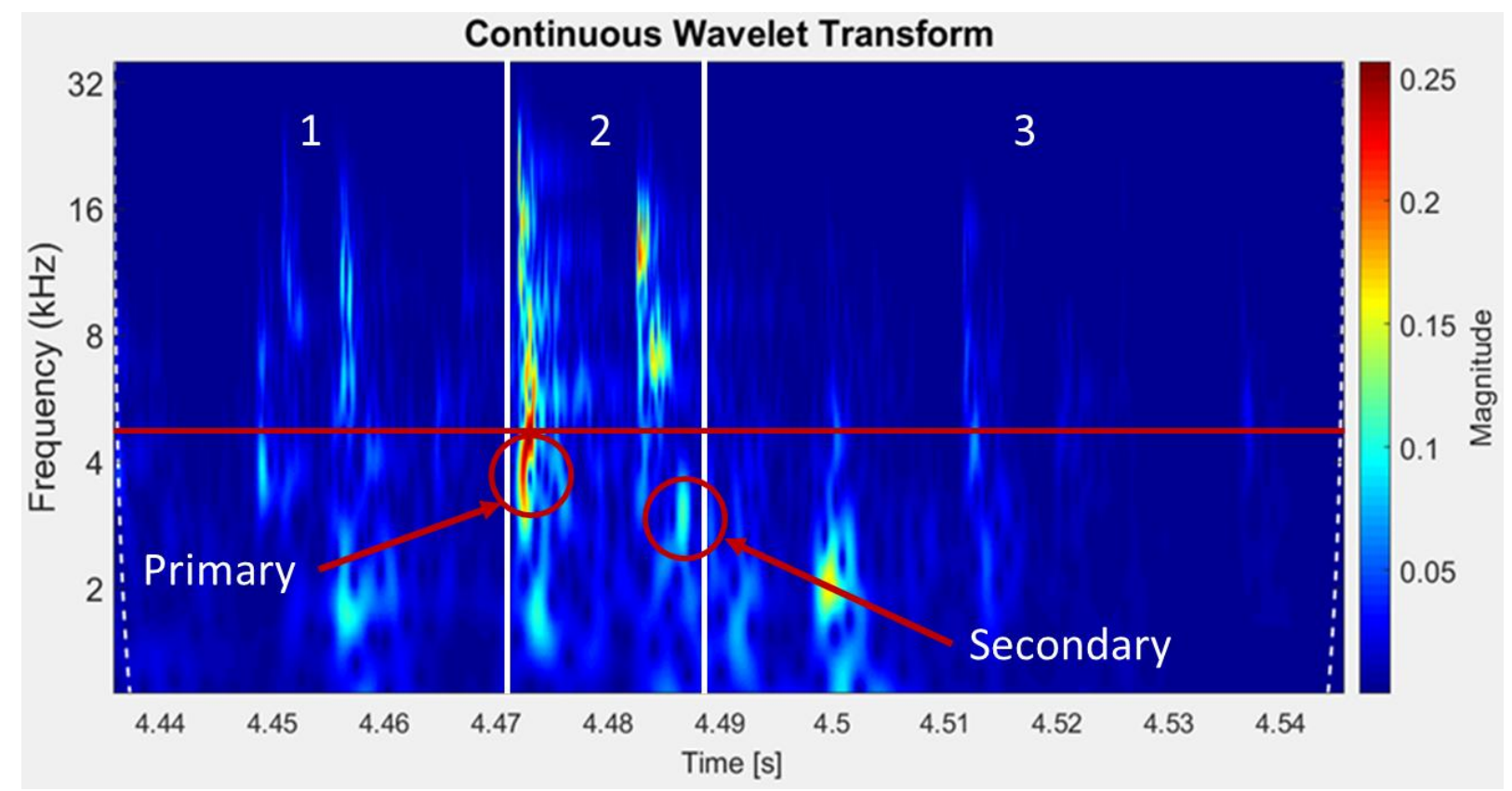

Figure 3.24: CWT of the $0.851 \mathrm{~m} / \mathrm{s}$ closure speed measured at MP2.

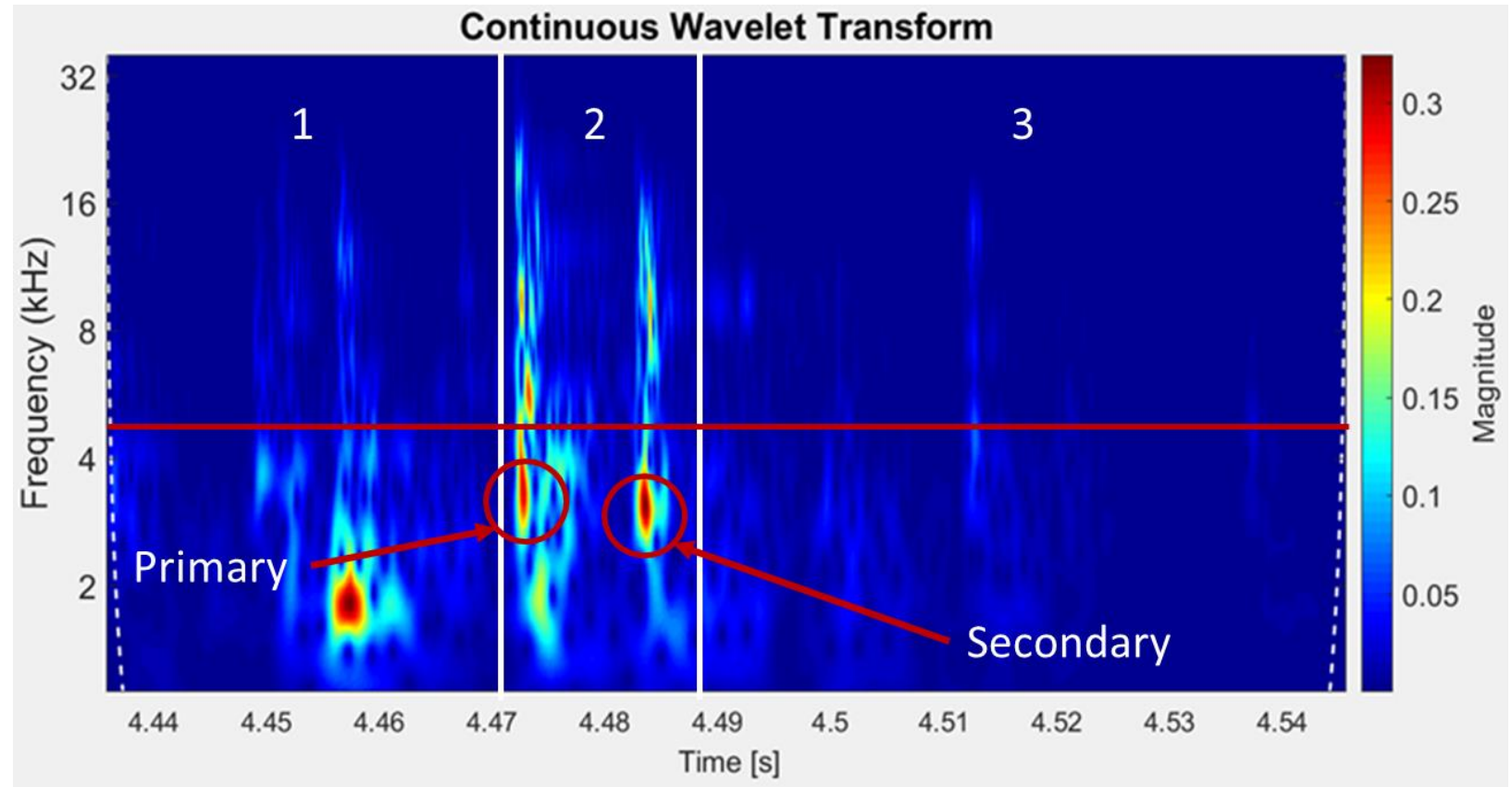

Figure 3.25: CWT of the $0.851 \mathrm{~m} / \mathrm{s}$ closure speed measured at MP3. 
Table 3.2: Approximate CWT Dominant Frequencies of the primary and secondary impacts.

\begin{tabular}{|c|c|c|c|}
\hline \multirow{2}{*}{ Entrance Speed [m/s] } & \multirow{2}{*}{ Microphone } & \multicolumn{2}{|c|}{ Frequency Range [kHz] } \\
\cline { 3 - 4 } & $\begin{array}{c}\text { Primary } \\
\text { Impact }\end{array}$ & $\begin{array}{c}\text { Secondary } \\
\text { Impact }\end{array}$ \\
\hline \multirow{3}{*}{$\mathbf{0 . 8 5 1}$} & MP1 & $3.7-5.0$ & $2.7-3.8$ \\
\cline { 2 - 4 } & MP2 & $3.6-5.0$ & $2.4-3.6$ \\
\cline { 2 - 4 } & MP3 & $2.3-4.0$ & $2.2-3.8$ \\
\hline \multirow{3}{*}{$\mathbf{1 . 1 7 9}$} & MP1 & $3.0-4.0$ & $0.5-1.5$ \\
\cline { 2 - 4 } & MP2 & $3.0-3.9$ & $1.7-2.5$ \\
\cline { 2 - 4 } & MP3 & $1.0-2.0$ & $2.1-2.9$ \\
\hline
\end{tabular}

A similar trend was observed with the CWT of the $1.179 \mathrm{~m} / \mathrm{s}$ impact speed. Figures 3.26-3.28 depict the wavelet analysis of the waveform shown in Figure 3.22. Similarly, comparing the timelines of Figure 3.22 and Figures 3.26-3.28 confirmed the primary and secondary impact events dominate the acoustic response. Table 3.2 displays the dominate frequency ranges for each $1.179 \mathrm{~m} / \mathrm{s}$ plot. The normalized magnitude of the dominant frequency of the primary impact exceeds the magnitude of the second impact for each trial. Additionally, it can be observed that the average difference in normalized magnitude of the primary impact shown in Figures 3.23-3.25 and Figures 3.26-3.28 is approximately 0.66.

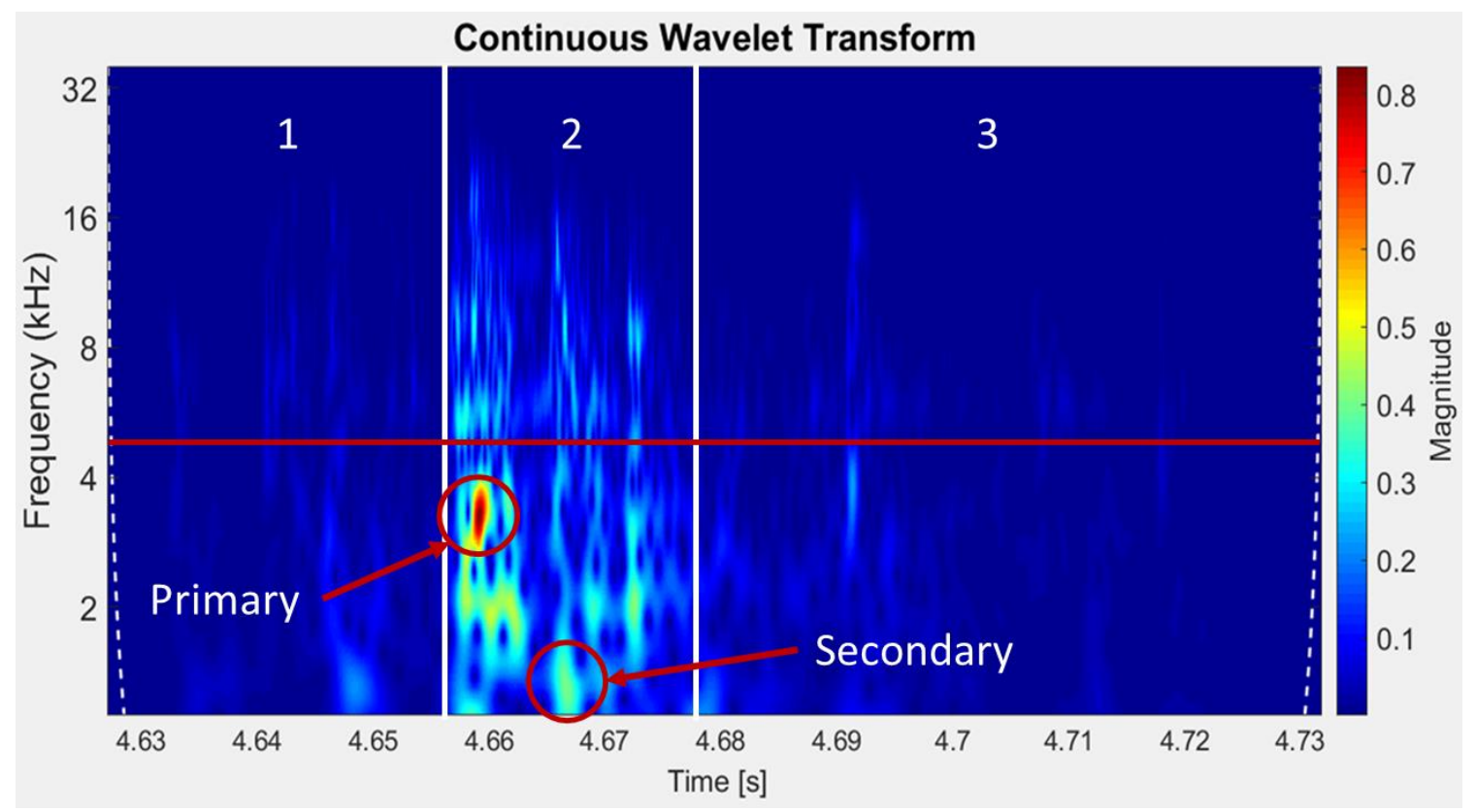

Figure 3.26: CWT of the $1.179 \mathrm{~m} / \mathrm{s}$ closure speed measures at MP1. 


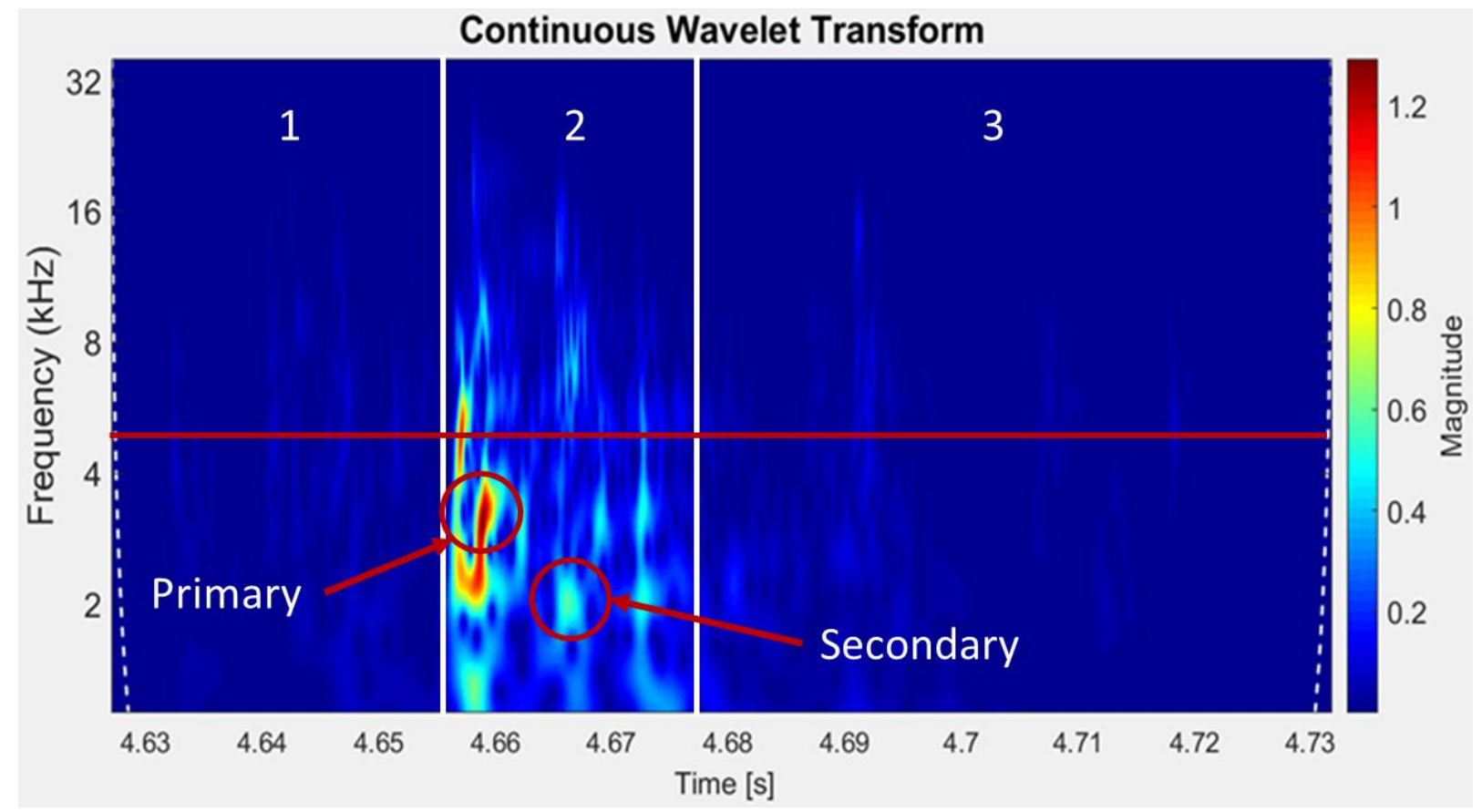

Figure 3.27: CWT of the $1.179 \mathrm{~m} / \mathrm{s}$ closure speed measures at MP2.

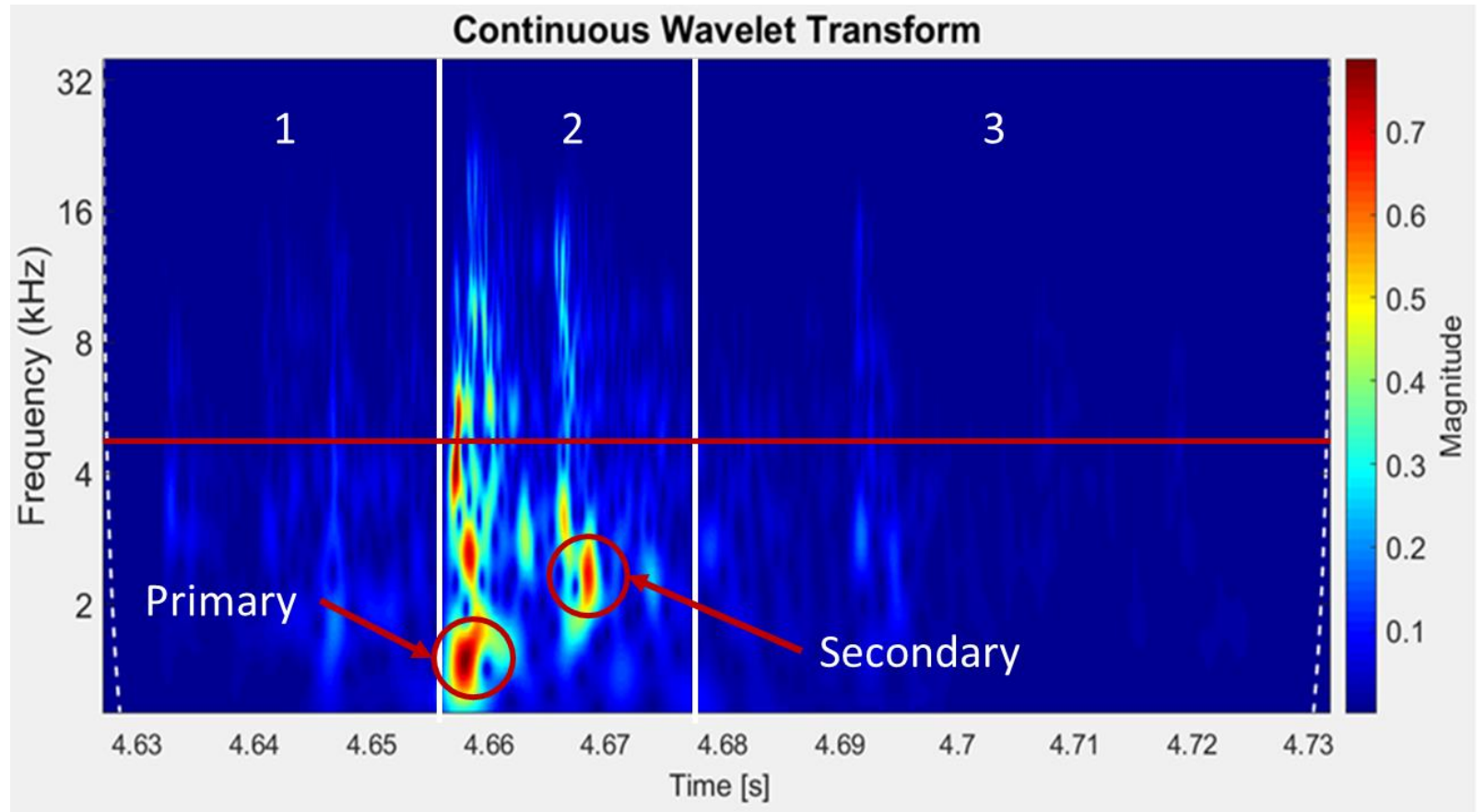

Figure 3.28: CWT of the $1.179 \mathrm{~m} / \mathrm{s}$ closure speed measures at MP3. 
By inspection, trends were evident as a result of the increased striker speed. Analysis of the CWT results indicated that the increase in speed permitted frequencies below $3 \mathrm{kHz}$ to become more pronounced in the audible response. The impulse from the reaction events caused these frequencies to resonate for a longer period of time. The observed increase in lower frequencies becoming more pronounced could be attributed to the additional kinetic energy introduced to the system by the faster closing speed.

Further comparison introduced a second observable trend. Figures 3.23-3.25 show the contribution of sounds from other latch component impacts not associated with the primary and secondary impacts. These "auxiliary" sounds occur in Segment 1 and Segment 3. Observing the results shown in Figures 3.26-3.28, the effect of these impacts was greatly minimized whereas the normalized magnitude of the impacts in Segment 2 increased. This finding suggests that changing the impacting speed from $0.851 \mathrm{~m} / \mathrm{s}$ to $1.179 \mathrm{~m} / \mathrm{s}$ has no effect on the energy present in the impact events occurring in Segment 1 and 3. Conversely, the increase in striker speed does affect the impacting events occurring in Segment 2. This finding is discussed in Chapter 4 where the dynamics of the latch components are investigated.

\subsubsection{Sound Pressure Level versus 1/3 Octave Band}

The calculation for the average SPL versus 1/3 octave band for the entire response was conducted using 1/3 octave band filters and incoherent average SPL addition (Equation 3.6). The data was collected from the 1/3 octave plots similar to Figure 3.20. The results from this procedure are presented in Figures 3.293.33 comparing the average SPL for each impact speed. Each plot represents the results of a single latch. Tables found in Appendix E compare the data presented in Figures 3.29-3.33 in addition to the percent difference of the frequency bands.

Further analysis indicated that the overall production of sound for the $0.851 \mathrm{~m} / \mathrm{s}$ closing speed showed dominant frequency bands of $1.6 \mathrm{kHz}$ and $4 \mathrm{kHz}$. This is indicative of the magnitude and duration of the frequencies depicted in the CWT. Changing the speed to $1.179 \mathrm{~m} / \mathrm{s}$ resulted in the $2 \mathrm{kHz}$ and $3.15 \mathrm{kHz}$ bands to dominate the acoustic response. However, with the increased duration of the lower frequencies 
succeeding the primary impact, frequency bands between $1.0 \mathrm{kHz}$ and $2 \mathrm{kHz}$ contributed significantly to the overall sound.

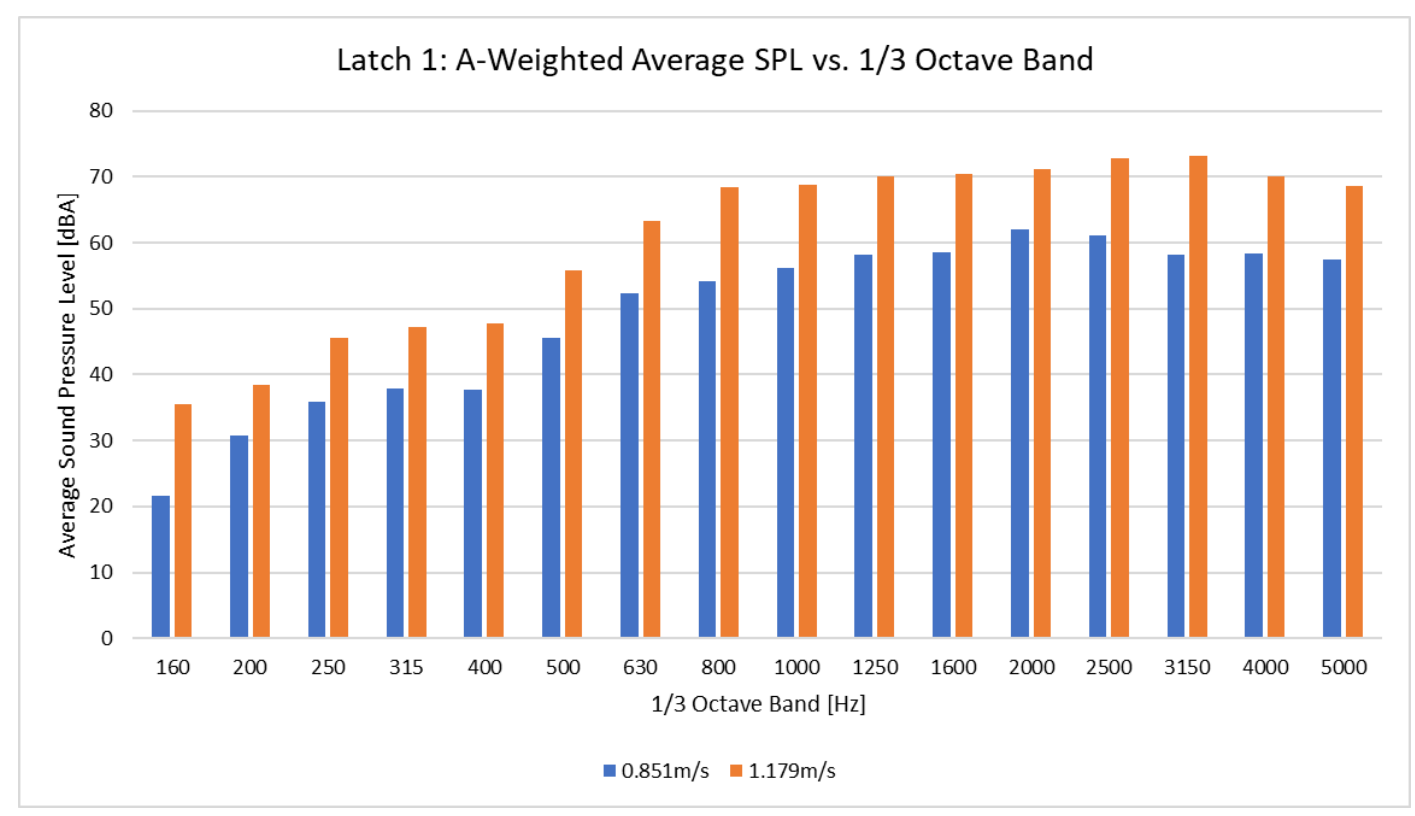

Figure 3.29: A-weighted average SPL versus 1/3 octave band of Latch 1.

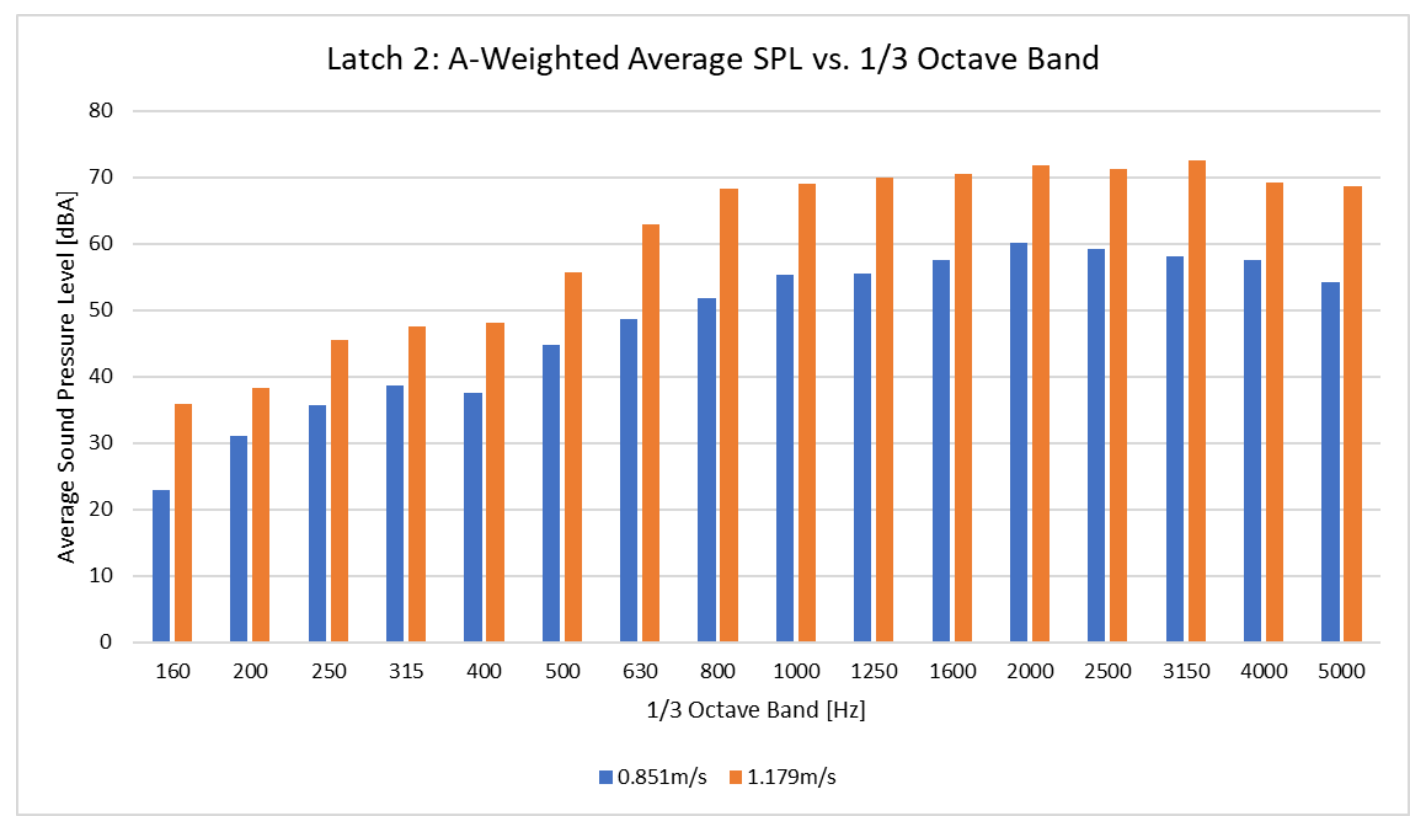

Figure 3.30: A-weighted average SPL versus 1/3 octave band of Latch 2. 


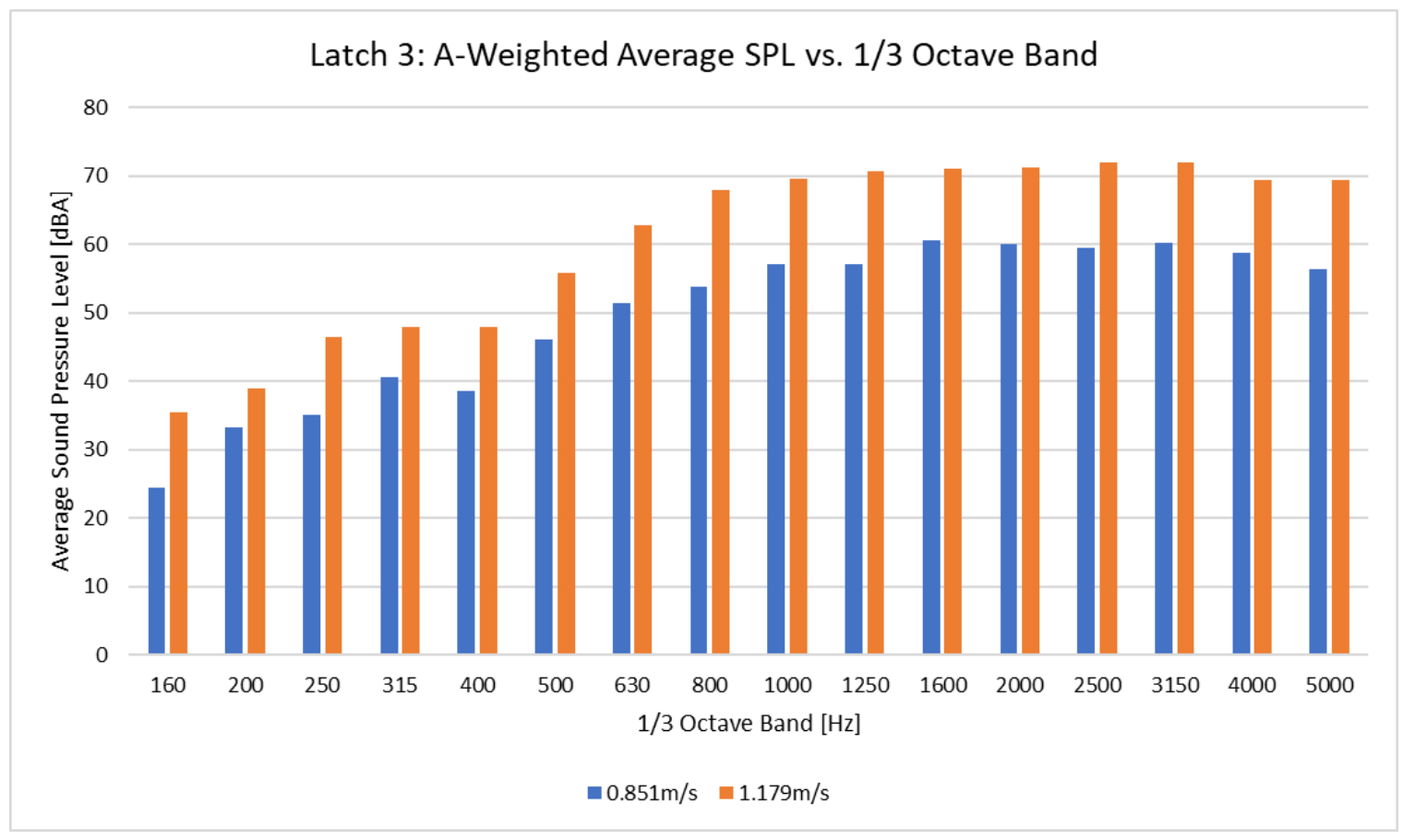

Figure 3.31: A-weighted average SPL versus 1/3 octave band of Latch 3.

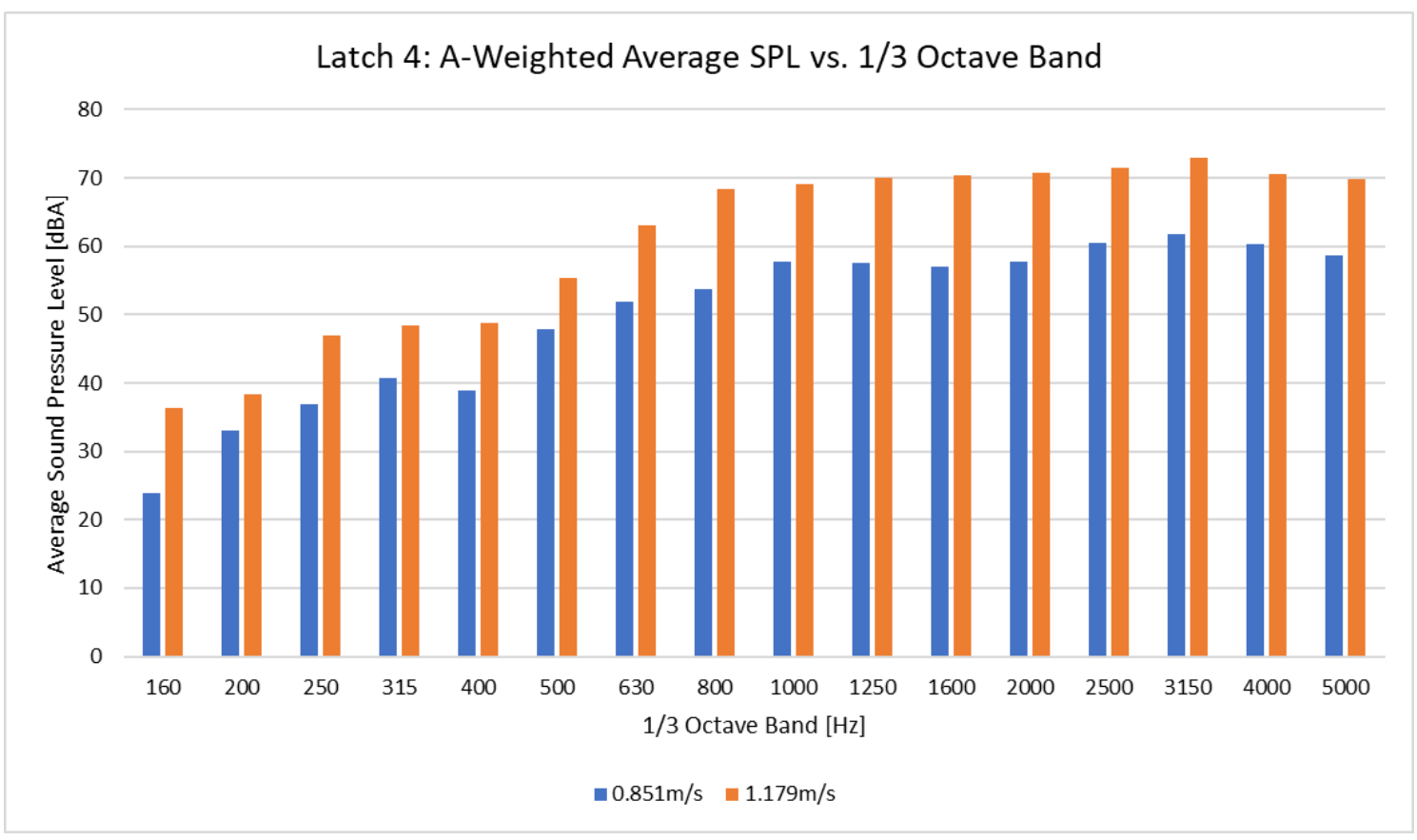

Figure 3.32: A-weighted average SPL versus 1/3 octave band of Latch 4 . 


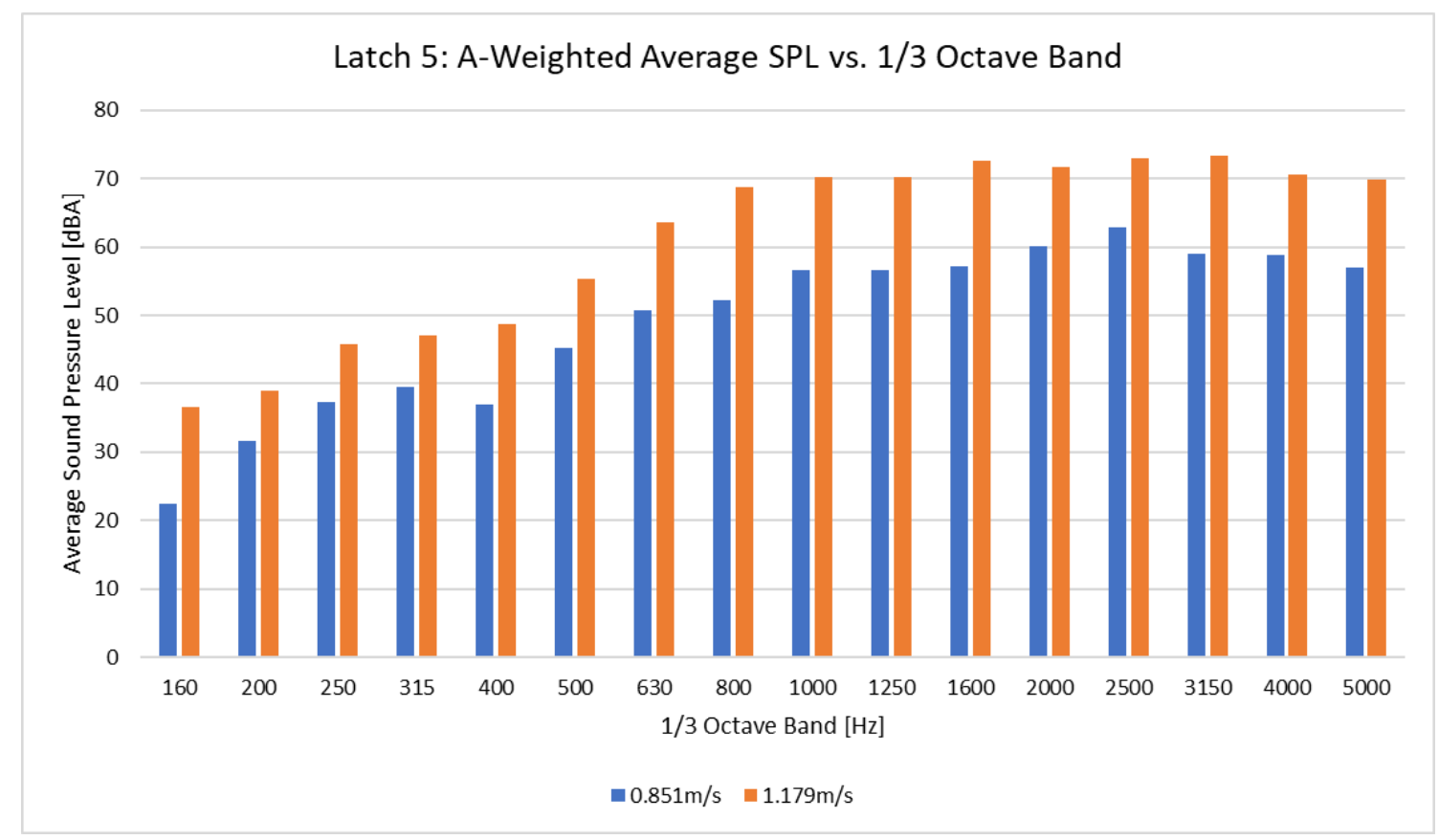

Figure 3.33: A-weighted average SPL versus 1/3 octave band of Latch 5.

It was evident that there was a significant difference in average SPL between the two speed conditions.

The additional energy presented to the system greatly influenced the acoustic response, resulting in each frequency band showing an increase in SPL. To obtain an overall average response, the results presented in Figures 3.29-3.33 were averaged using Equation 3.6. Figure 3.34 exhibits the A-weighted average SPL versus a 1/3 octave bandwidth of all latch samples. Additionally, Tables 3.3 and 3.4 display the results shown in Figure 3.34 in addition to the standard deviation and standard error. The percentage difference in average SPL between the two test conditions is displayed in Table 3.5. From this the average SPL difference between the two test speeds was calculated to be $11.35 \mathrm{dBA}$. 


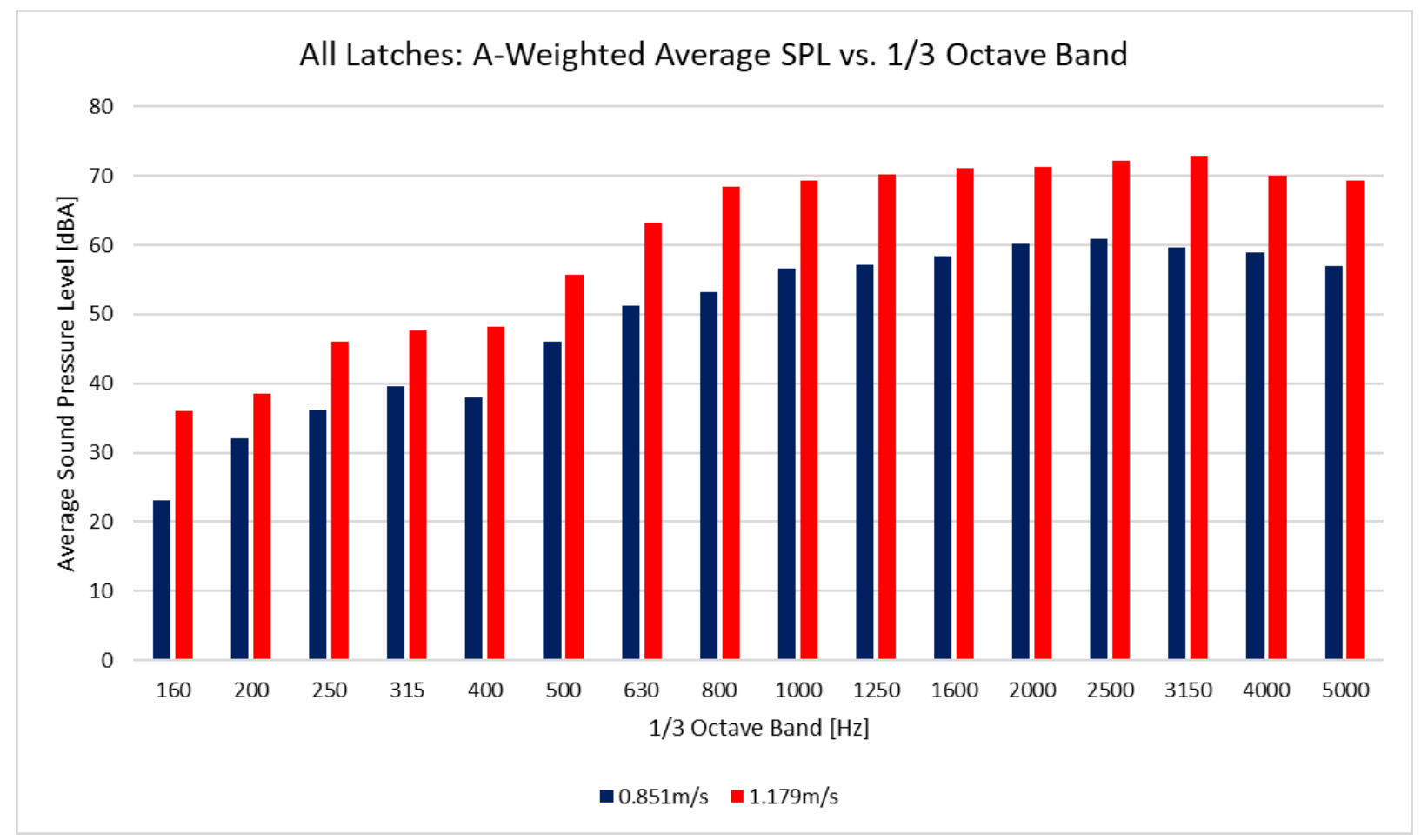

Figure 3.34: A-weighted average SPL versus 1/3 octave band of all latches.

Table 3.3: Tabular average SPL results of the $0.851 \mathrm{~m} / \mathrm{s}$ closure speed.

\begin{tabular}{|c|c|c|c|}
\hline $\mathbf{1 / 3}$ Octave Band & SPL [dBA] & Standard Deviation & Standard Error \\
\hline $\mathbf{1 6 0}$ & 23.18 & 1.15 & 0.51 \\
\hline $\mathbf{2 0 0}$ & 32.07 & 1.09 & 0.49 \\
\hline $\mathbf{2 5 0}$ & 36.25 & 0.92 & 0.41 \\
\hline $\mathbf{3 1 5}$ & 39.63 & 1.25 & 0.56 \\
\hline $\mathbf{4 0 0}$ & 38.00 & 0.80 & 0.36 \\
\hline $\mathbf{5 0 0}$ & 45.99 & 1.20 & 0.54 \\
\hline $\mathbf{6 3 0}$ & 51.24 & 1.44 & 0.64 \\
\hline $\mathbf{8 0 0}$ & 53.22 & 1.06 & 0.48 \\
\hline $\mathbf{1 0 0 0}$ & 56.69 & 0.91 & 0.41 \\
\hline $\mathbf{1 2 5 0}$ & 57.13 & 1.02 & 0.45 \\
\hline $\mathbf{1 6 0 0}$ & 58.41 & 1.48 & 0.66 \\
\hline $\mathbf{2 0 0 0}$ & 60.25 & 1.57 & 0.70 \\
\hline $\mathbf{2 5 0 0}$ & 60.84 & 1.44 & 0.64 \\
\hline $\mathbf{3 1 5 0}$ & 59.69 & 1.58 & 0.71 \\
\hline $\mathbf{4 0 0 0}$ & 58.86 & 1.02 & 0.46 \\
\hline $\mathbf{5 0 0 0}$ & 56.99 & 1.61 & 0.72 \\
\hline & & & \\
\hline
\end{tabular}


Table 3.4: Tabular average SPL results of the $1.179 \mathrm{~m} / \mathrm{s}$ closure speed.

\begin{tabular}{|c|c|c|c|}
\hline $\mathbf{1 / 3}$ Octave Band & SPL [dBA] & Standard Deviation & Standard Error \\
\hline $\mathbf{1 6 0}$ & 35.99 & 0.46 & 0.21 \\
\hline $\mathbf{2 0 0}$ & 38.60 & 0.34 & 0.15 \\
\hline $\mathbf{2 5 0}$ & 46.10 & 0.60 & 0.27 \\
\hline $\mathbf{3 1 5}$ & 47.70 & 0.57 & 0.25 \\
\hline $\mathbf{4 0 0}$ & 48.25 & 0.44 & 0.20 \\
\hline $\mathbf{5 0 0}$ & 55.63 & 0.23 & 0.10 \\
\hline $\mathbf{6 3 0}$ & 63.18 & 0.32 & 0.14 \\
\hline $\mathbf{8 0 0}$ & 68.35 & 0.31 & 0.14 \\
\hline $\mathbf{1 0 0 0}$ & 69.39 & 0.56 & 0.25 \\
\hline $\mathbf{1 2 5 0}$ & 70.15 & 0.30 & 0.13 \\
\hline $\mathbf{1 6 0 0}$ & 71.10 & 0.94 & 0.42 \\
\hline $\mathbf{2 0 0 0}$ & 71.35 & 0.46 & 0.20 \\
\hline $\mathbf{2 5 0 0}$ & 72.16 & 0.81 & 0.36 \\
\hline $\mathbf{3 1 5 0}$ & 72.83 & 0.56 & 0.25 \\
\hline $\mathbf{4 0 0 0}$ & 69.98 & 0.67 & 0.30 \\
\hline $\mathbf{5 0 0 0}$ & 69.35 & 0.64 & 0.29 \\
\hline
\end{tabular}

Table 3.5: Percent difference in average SPL between the $0.851 \mathrm{~m} / \mathrm{s}$ and $1.179 \mathrm{~m} / \mathrm{s}$ closure speeds.

\begin{tabular}{|c|c|}
\hline 1/3 Octave Band & Percent Difference [\%] \\
\hline $\mathbf{1 6 0}$ & 55.27 \\
\hline $\mathbf{2 0 0}$ & 20.39 \\
\hline $\mathbf{2 5 0}$ & 27.16 \\
\hline $\mathbf{3 1 5}$ & 20.37 \\
\hline $\mathbf{4 0 0}$ & 26.96 \\
\hline $\mathbf{5 0 0}$ & 20.96 \\
\hline $\mathbf{6 3 0}$ & 23.29 \\
\hline $\mathbf{8 0 0}$ & 28.42 \\
\hline $\mathbf{1 0 0 0}$ & 22.41 \\
\hline $\mathbf{1 2 5 0}$ & 22.79 \\
\hline $\mathbf{1 6 0 0}$ & 21.73 \\
\hline $\mathbf{2 0 0 0}$ & 18.42 \\
\hline $\mathbf{2 5 0 0}$ & 18.61 \\
\hline $\mathbf{3 1 5 0}$ & 22.01 \\
\hline $\mathbf{4 0 0 0}$ & 18.89 \\
\hline $\mathbf{5 0 0 0}$ & 21.68 \\
\hline
\end{tabular}




\subsubsection{Sound Quality Metrics: Loudness and Sharpness}

From the data presented in both analyses, an inference can be made on the loudness and sharpness of the recorded samples. As described in Chapter 2, loudness is the acoustic descriptor used to place a sound on a scale ranging from soft to loud. It is mainly influenced by the sound pressure level [23]. The observed correlation between the two impact speeds indicate larger SPL levels generated by the faster closing speed. As expected, this finding suggests that the faster speed caused an increase in the perceived loudness of the door closing procedure which could affect the impression of the sound heard by the consumer. Returning to the study performed by Kuwano et al., it was observed that an increase in loudness does not always result in a sound that is less preferred to the user. Some individuals may have a preference to a door closing sound that is louder over one that is softer. It is speculated that sounds with greater loudness levels may influence an individual's perception of the vehicle assembly being robust and able to withstand harsh environments without failure. Additionally, the results of the same study insinuated that sharpness has a greater influence on the sound quality of a door latch compared to loudness.

Applying this finding to the current research, one can infer that an increase in loudness does not directly influence the perception of the sound. Instead, the increase in loudness allows the lower frequencies present in the primary and secondary impacts to become more prominent. In accordance with the generally accepted viewpoint of frequency preferences, simply increasing the entrance speed of the striker may produce a more preferred sound due to the increase in magnitude and duration of the lower frequencies within the sound. This is by no means an absolute solution, but it is an unexpected outcome that could introduce an approach to sound generation that is still unexplored. 


\subsection{Conclusion}

Overall, the comparison of the experimental waveforms at each microphone position provided excellent insight into the frequency composition of the acoustic phenomena. As expected, slight variation in the recorded sound pressure at each receiver was present due to the given boundary conditions. Based on the results of the CWT analyses of both impact speeds, it was determined that the impact speed of the striker influenced the acoustic response. In this case, faster speeds introduced more energy into the system, producing lower dominant frequencies with a much greater magnitude during the primary impact.

Inferences were made based on the CWT results and 1/3 octave SPL results. In relation to what has already been observed in psychoacoustic research, it is assumed that the sharpness characteristic of the D21MC sound has a greater impact on the sound quality compared to loudness. This is based on the fact that consumers are more inclined to purchase consumer products that they believe sound "better" [39][25]. However, the concept of what sounds "better" differs for each individual.

Psychoacoustic principles coupled with sound quality metrics suggest that increasing the speed from $0.851 \mathrm{~m} / \mathrm{s}$ to $1.179 \mathrm{~m} / \mathrm{s}$ produces a more "preferred" sound due to the more pronounced lower frequencies. Although this is not a definitive solution to making the latch sound better, it is interesting to note the counter-intuitive effect closing speed has on the acoustic response with the potential of making future vehicle designs sound better with little modification. 


\section{Chapter 4}

\section{Computational Procedures and Analysis}

\subsection{Computational Analysis and Methodology}

A computational analysis was performed to compliment the experimental portion of this study.

Experimental testing can be an arduous and expensive process depending on the testing method, the complexity of the model and the testing environment. A computational analysis is capable of eliminating the need for repetitive and expensive testing practices by simulating the real-life test setting within a virtual environment. These models allow design engineers to modify features of a product to obtain a result without the need to completely fabricate the new part in real life. For these reasons, computational simulations have become a common fixture in engineering design

Finite Element Analysis (FEA) has been used in industry for many years due to its reliability and accuracy with regard to mechanical dynamics and transient impacts [11][41]. When performed correctly, acoustic simulations can be a powerful tool in the development stages of a product. However, correct formulation of transient acoustic computational analyses can be a tedious task. This chapter explores the steps taken to investigate the mechanics of the impact events occurring within the D21MC door closure during operation. The dynamic results aided in the formulation of a transient acoustic analysis adhering to industry accepted methodologies. These methodologies were adopted from various ANSYS Workbench user guides in addition to the works published by Cazzolato et al. [11] and Von Estorff [28]. The contents of this chapter have been adapted from a peer-reviewed conference paper entitled "Experimental and Computational Methods for Investigating Automotive Door Closure Sounds"[31]. Within this section, the terms "pawl" and "ratchet" refer to the bonded coupling of the "pawl and pawl encapsulation" as well as the "ratchet and ratchet encapsulation" respectively. 


\subsection{Acoustic Simulation Workstation}

Complex acoustic simulations like the one investigated require a powerful workstation. The workstation used in this investigation contained 128 gigabytes of RAM DDR4-2132 (1066MMHz), 4 gigabytes of dedicated graphics, 2 terabytes of hybrid storage (SSD and HDD) as well as an intel Core i7 6850k chip $(3.60 \mathrm{GHz})$.

\subsection{Acoustic Simulation Methodologies}

In acoustic computational analysis, there are three common methodologies used in industry, each with its own advantages and disadvantages. The most common numerical analyses are the element-based methodologies such as the Finite Element Method (FEM) and the Boundary Element Method (BEM). FEM and BEM are commonly employed when dealing with frequencies within the lower to medium range [28]. Statistical Energy Analysis (SEA) is commonly used in the higher frequency range where the contribution to the aural response is determined by calculating the flow and storage of vibrational energy within the acoustic source [30]. For this study, several factors were considered in determining the correct approach to acoustic modelling: the workstation available for computational analysis; the complexity of the latch model; and the frequency band of interest.

Given the nature of the investigation, it was important that the method chosen was able to model the frequencies at the lower end of the audible spectrum. It is understood that both FEM and BEM excel in the mid to low region whereas SEA is known to be less reliable [28]. Therefore, it was concluded that SEA was not an acceptable methodology for this particular study.

\subsubsection{Finite Element Method}

The Finite Element Method is a domain type methodology which requires the entire computational model to be discretized into elements. This includes the structural model as well as the surrounding acoustic fluid domain [28][11]. FEM is the most common computational methodology used in industry, not just in acoustic design but in electrical, structural and fluid dynamics design as well. Finite Element Analysis 
(FEA) is also capable of solving radiating acoustic problems using "infinite" acoustic elements. This element type is able to simulate exterior acoustics efficiently and effectively [28][11]. To clarify, acoustic literature defines "infinite" or "infinity" elements as boundary elements with the ability to allow radiating sound pressure to travel away from the sound source without being reflected back at the acoustic fluid boundary [11][42]. This feature is analogous to the simulation of a free field (FF) environment. A description of an FF environment was provided in Chapter 2. This acoustic formulation is also adept at modeling transient acoustic phenomena, a characteristic well suited for this investigation [28].

\subsubsection{Boundary Element Method}

The Boundary Element Method benefits from using boundary type formulations as it requires only the surface boundary of an acoustic medium to be discretized [28]. The premise of BEM is to express the physical acoustic phenomena (radiating sound waves) by physical qualities like sound pressure and velocity, measured at the fluid boundaries. The benefit of using BEM is the significant decrease in body discretization as opposed to FEM; only the surface of the investigated body needs to be discretized. This is a useful feature for bodies with complex geometry as described in the study mentioned in Chapter 2.

Additionally, the radiation of the sound waves to "infinity" is implied within the BEM formulation, preventing the need to define infinite elements in the acoustic media [28].

Although there are many acoustic software packages available in the market, this study was limited to the use of the ANSYS software package which is only capable of performing acoustic finite element analyses natively. This was not a major concern as the literature suggested that the finite element method is better suited for transient acoustic phenomena [28][11]. Therefore, all the computational results presented in this work are from FEA alone.

\subsubsection{Upper Frequency Limit}

It is understood that as frequency increases, the number of modes in the system increases as well. These modes become increasingly more difficult to model using traditional finite element practices requiring a 
large number of elements. The same is said for computational acoustic analysis where the modelling of the sound pressure at higher frequencies becomes more difficult to simulate. This increased difficulty can also contribute to reduced accuracy of the predicted sound. As a general rule of thumb, discretized acoustic media should contain a minimum of 6 to 12 elements per wavelength depending on the shape function used [11]. This is discussed further in a subsequent section. As a result, an upper limit of $5 \mathrm{kHz}$ was used in this work. Applying this limit ensured accurate measurement of the surface vibrations and of the latch components investigated in addition to the predicted acoustic phenomena.

\subsection{ANSYS Workbench}

ANSYS 17.2 Workbench simulation software was the primary software used to perform the computational portion of this study. An academic license was acquired enabling all simulation modules and removing any element and node restrictions. The following ANSYS Workbench modules used were: SpaceClaim (SC), Design Modeler (DM), Rigid Body Dynamic (RBD), Explicit Dynamic (ED), and Transient Structural (TS). The tasks performed in each module are described in detail in the following sections. An effort was made to export the kinematic motion of the latch components into a single acoustic analysis where the dynamic response and the acoustic response of the system could be measured. However, the ANSYS was not able to perform a simulation of this type which lead to the decision of separating the response of the latch into three separate simulation modules.

The material properties mentioned in Chapter 2 were used for each computational analysis. This analysis investigated the impact events occurred during latch operation. As a result, only the pawl, ratchet, striker, over-slam bumpers and housing were computationally investigated. The remaining latch components that were not involved with the striker/latch engagement were omitted.

\subsubsection{ANSYS ACT Acoustic Extension}

In order for ANSYS to generate a suitable environment for the D21MC latch acoustic analysis, an additional software extension was required. The acoustic extension was downloaded from the ANSYS 
Application Customization Toolkit (ACT) webstore [43]. After installation, the acoustics toolbar was added to the TS module. This toolbar allowed for the creation of acoustic fluid bodies, a boundary between the latch structure and acoustic media, in addition to virtual microphone receivers.

\subsection{SpaceClaim}

SpaceClaim is a computer aided design (CAD) software included in the ANSYS software package.

SpaceClaim was used to prepare the door closure computational model for analysis. Magna Closures Inc. provided a detailed CAD model of the D21MC door system (latch mechanism and striker) and pendulum apparatus used for this study. Figure 4.1 depicts the door closure system model prior to dynamic and acoustic analysis. Preparation of the latch involved the removal of the double pendulum model in addition to repositioning the latch and striker closer to the point of origin such that the x-z plane was in line with the center plane of the striker. The striker was located to this position to facilitate the definition of the striker linear actuation in future analyses. The repositioned striker and latch can be seen in Figure 4.2. The newly positioned latch was exported as a parasolid file and imported to ANSYS DM for further processing. Figure 4.2 depicts the entirety of the latch and its components. Using SpaceClaim, the dimensions of the door closure mechanism excluding the striker are $0.168 \mathrm{~m}, 0.0966 \mathrm{~m}$ and $0.0906 \mathrm{~m}$ denoting the length, width and height, respectively.

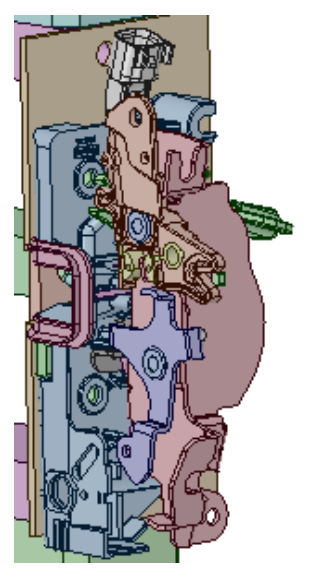

Figure 4.1: Fully assembled CAD model of the door closure system. 


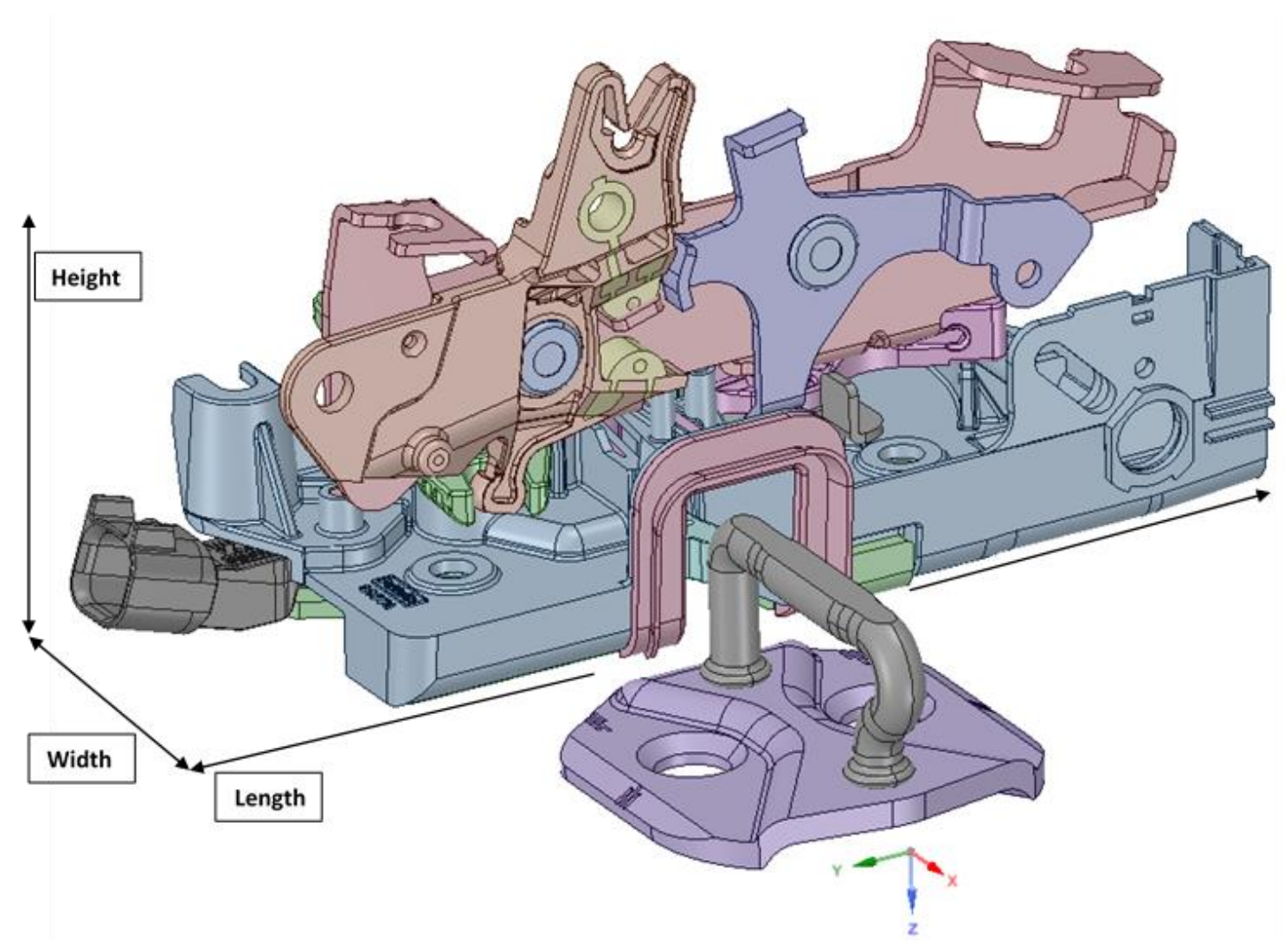

Figure 4.2: Fully assembled and repositioned door closure system.

\subsection{Design Modeler: Structural Pre-Processing}

Design Modeler was used to simplify the latch component geometry prior to analysis. Fillets used to soften edges were removed, allowing for a more 'regular-shaped' geometry to improve overall mesh quality. An example of this action is viewed in Figure 4.3 and Figure 4.4. It is common practice for irregular shaped geometry to possess many faces 'stitched' together to form a complete part. An example of this is the striker component shown in Figure 4.5. However, part generation using this method can cause inadequate mesh generation. To minimize the possibility of a poorly generated mesh, the simplified striker faces were merged resulting in larger and smoother faces (Figure 4.5). 

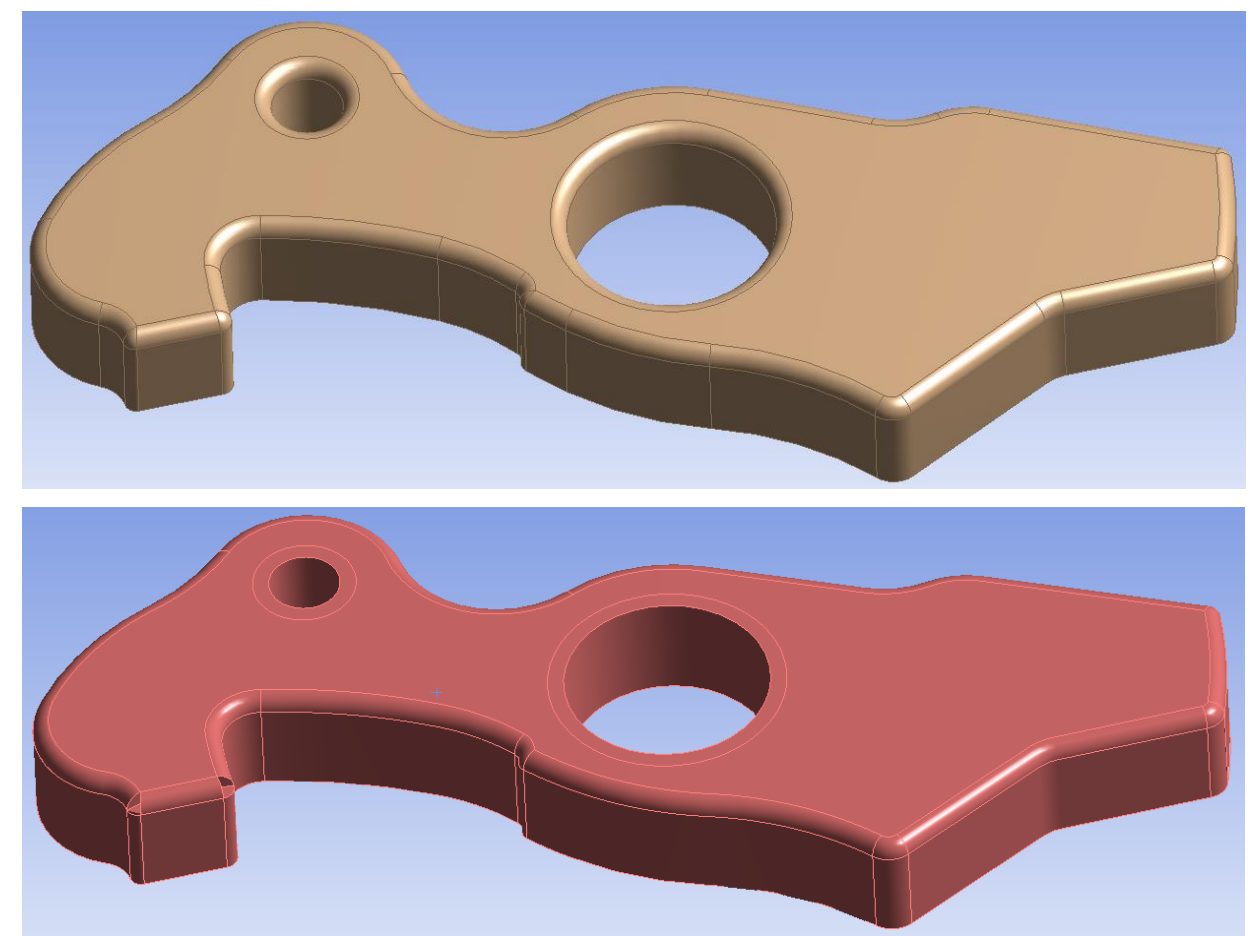

Figure 4.3: Original pawl insert (Top). Simplified, multi-body pawl insert (Bottom).
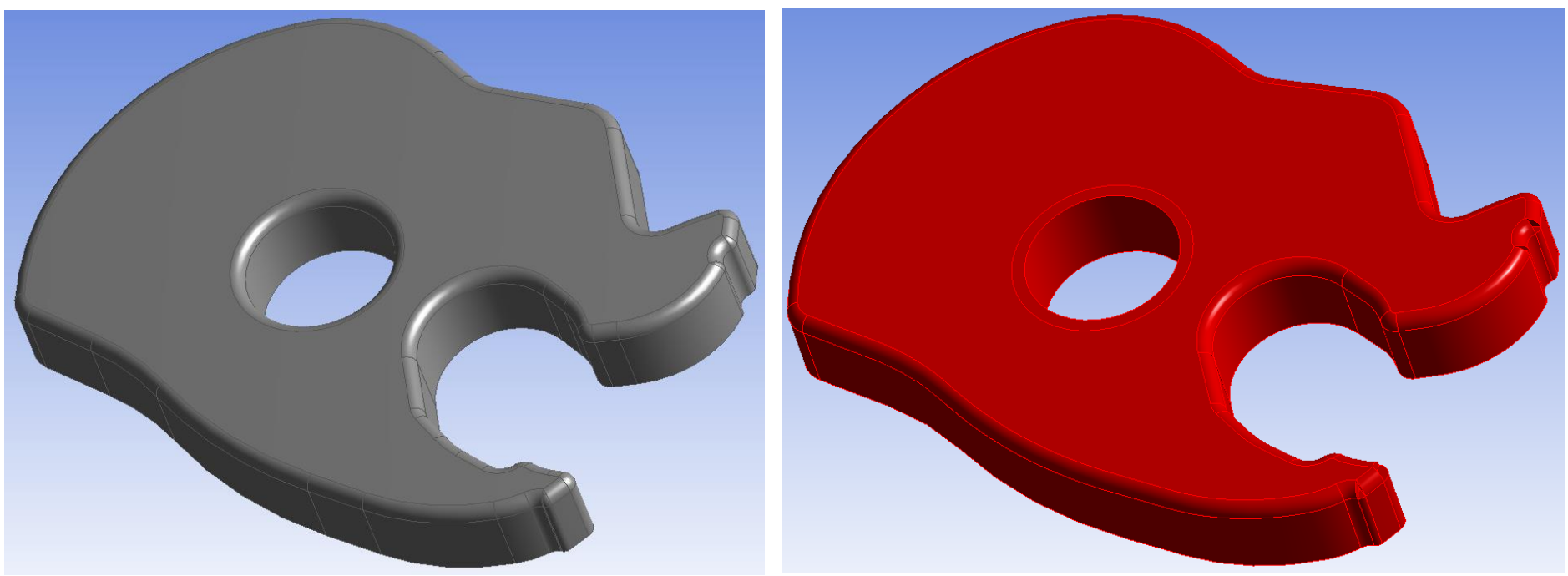

Figure 4.4: Original ratchet insert (Left). Simplified, multi-body ratchet insert (Right). 

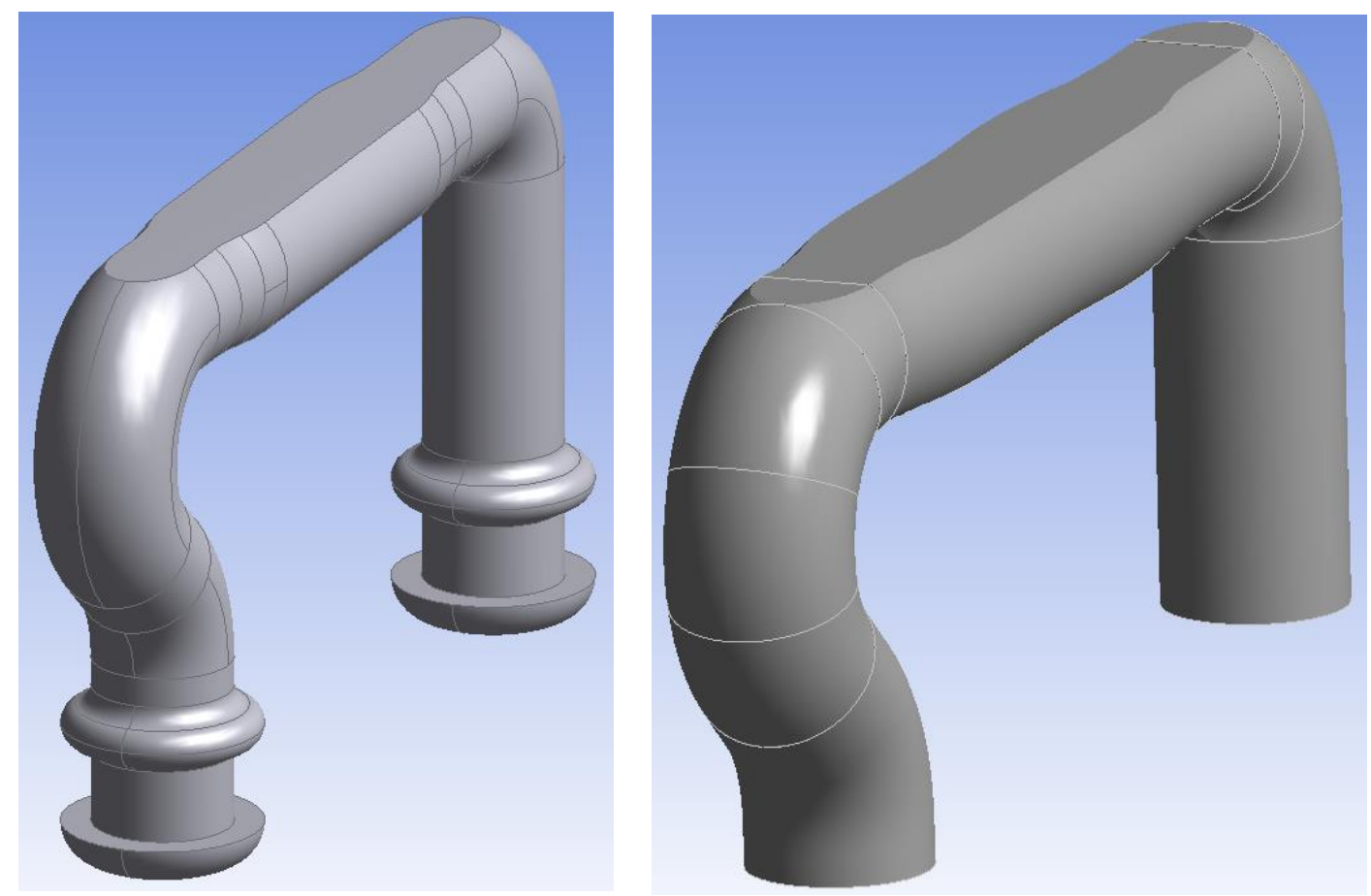

Figure 4.5: Original striker (Left). Simplified, multi-body striker (Right).

Due to the highly irregular shape of the striker (highly curved and uneven surfaces), a good quality mesh was difficult to achieve. Further simplification of the latch was performed to remove the bottom portion of the striker used to mount itself to the vehicle pillar. This simplification resulted in a better quality mesh of the part in addition to the mesh quality of the surrounding acoustic fluid region (discussed in a subsequent section). In addition, the latch components investigated were divided into multiple 'smaller' and 'regular-shaped' bodies which summed together to form a complete part denoted as a "multi-body part". The result of this procedure can be seen in Figures 4.3-4.5. This facilitated a better mesh generation of the whole part as discussed in a subsequent section.

The original component geometry possessed 'highly curved, slivered' sections which would not comply with the desired meshing method needed for the ED and TS analysis. Due to the small volume of these slivered bodies, it was concluded that the effect of their removal was negligible compared to the poor- 
quality mesh that would be generated due to their inclusion in the analysis. This is shown in Figures 4.34.5 as well.

\subsection{Rigid Body Dynamics}

Four distinct impact events were investigated to determine their influence on the radiated acoustic phenomena. Previous research has indicated the importance of analyzing the dynamic behavior of components to better understand their contribution to the overall sound [27]. During the data collection process, it was difficult to accurately collect surface velocity measurements on the latch body due to the complex design of the closure system being investigated; the short-transient nature of the latch closing process; as well as the dynamics of the testing apparatus. Therefore, a computational method using Rigid Body Dynamics (RBD) was chosen to approximate the complex component interactions of the D21MC latch at the predefined striker entrance speeds.

\subsubsection{Coil Spring to Torsional Spring Conversion}

The door closure used two coil springs to actuate the pawl and ratchet motion to achieve a fully latched position. However, coil springs could not be used within the computational model due to the geometry of the real-life model. As seen in Figure 4.6, the pawl and ratchet spring closely follow the circumference of a circle with a constant radius. To correctly model the motion of the latch components, the forces of the coil springs were replicated using equivalent torsional springs within the RBD module. Calculations were performed to determine the equivalent torsional spring constant as well as the installed torque (pre-load) during operation. The results of the conversion are displayed in Table 4.1. The procedure is described in detail in Appendix F. The stability of the kinematic model was greatly improved by including spring element damping. A spring damping value of $c=5 \mathrm{E}-4 \mathrm{Nms} / \mathrm{rad}$ provided the most accurate representation of the real ratchet and pawl behavior. 


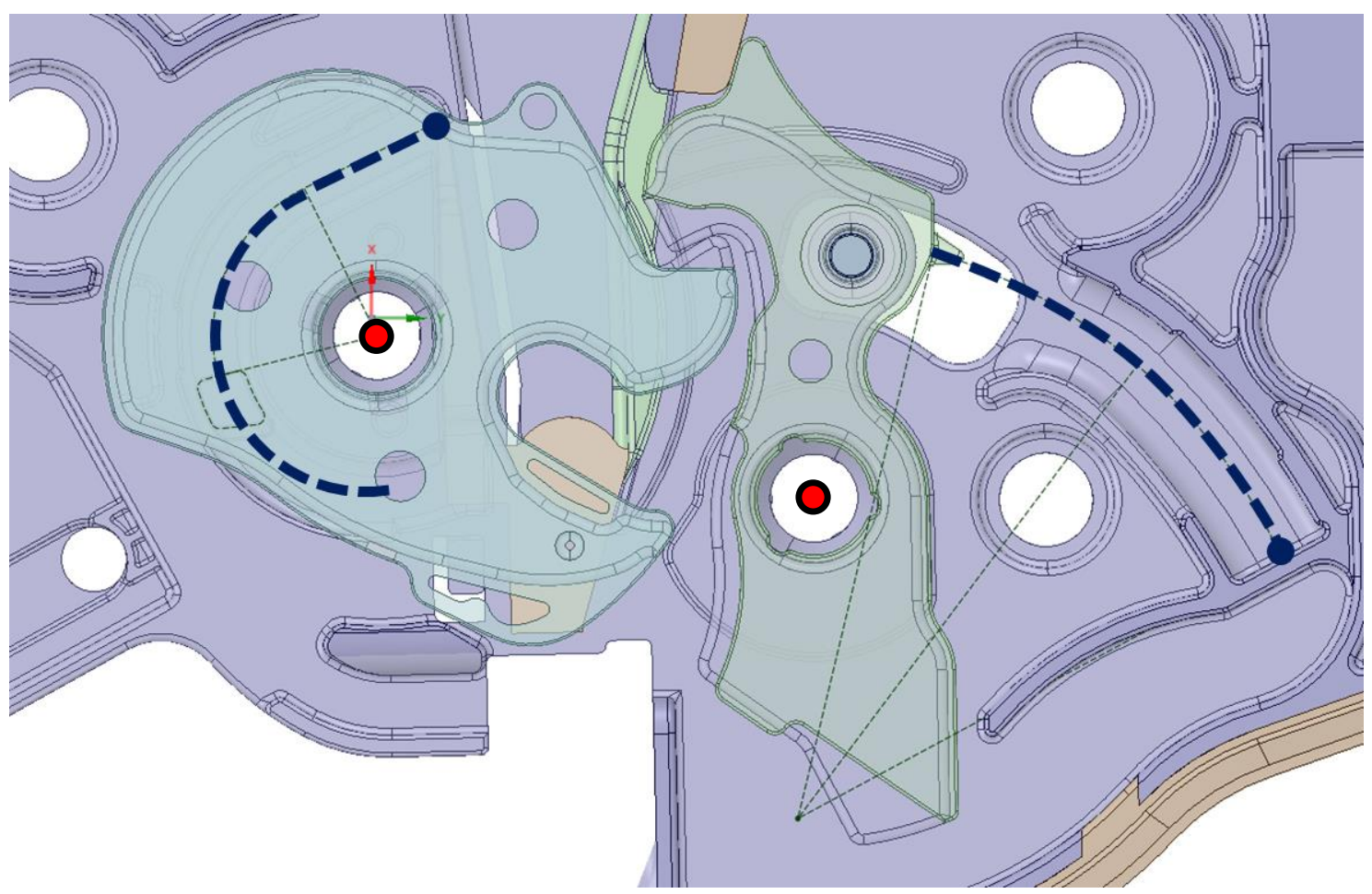

Figure 4.6: Pawl and ratchet coil spring travel path during latch operation.

Table 4.1: Summary of coil to torsional spring conversion.

\begin{tabular}{|c|c|c|}
\cline { 2 - 3 } \multicolumn{1}{c|}{} & Pawl & Ratchet \\
\hline Coil Spring Constant & $537.9 \frac{\mathrm{N}}{\mathrm{m}}$ & $328 \frac{\mathrm{N}}{\mathrm{m}}$ \\
\hline $\begin{array}{c}\text { Equivalent Torsional } \\
\text { Spring Constant }\end{array}$ & $0.8245 \frac{\mathrm{Nm}}{\mathrm{rad}}$ & $0.0426 \frac{\mathrm{Nm}}{\mathrm{rad}}$ \\
\hline Installed Torque & $0.27 \mathrm{Nm}$ & $0.0479 \mathrm{Nm}$ \\
\hline
\end{tabular}

\subsubsection{Model Assembly}

A revolute joint was used in $\mathrm{RBD}$ to allow the ratchet and pawl to rotate about a fixed point. This point is depicted in Figure 4.6 by the red marker. As previously mentioned, the latches used in the experimental analysis were fabricated the day of testing. The new latches were assembled and lubricated to specifications. With newly applied lubrication, it was assumed the frictional effects of the rotating pawl 
and ratchet were negligible. This assumption was carried over to the RBD computational analysis where the pawl and ratchet revolute joints were defined to be frictionless.

A translational joint was used to define the entrance speed of the striker relative to the latch. Two separate analyses were performed at $0.851 \mathrm{~m} / \mathrm{s}$ and $1.179 \mathrm{~m} / \mathrm{s}$, matching the speeds used in the experimental analysis. The contact surfaces of the latch components were meshed using quadrilateral shaped elements with mid-side nodes enabled (SURF154 element) as it is one of the most common element types used for 3D RBD analyses [44]. The element size used for the contact surface was $1.8 \mathrm{E}-4 \mathrm{~m}$. The average time step was $1.0 \mathrm{E}-6 \mathrm{~s}$.

\subsubsection{Results and Analysis}

Velocity probes were positioned at each of the impact locations to record the tangential speed of the component prior to impact. Figure 4.7 demonstrates the tracked speed from the probe positioned at the end of the pawl encapsulation to record the speed prior to impact with the bumper. Only the magnitude of the velocity was recorded as the direction vector was determined to be normal to each impacting surface. Table 4.2 shows the measured speeds of each impact event in sequential order.

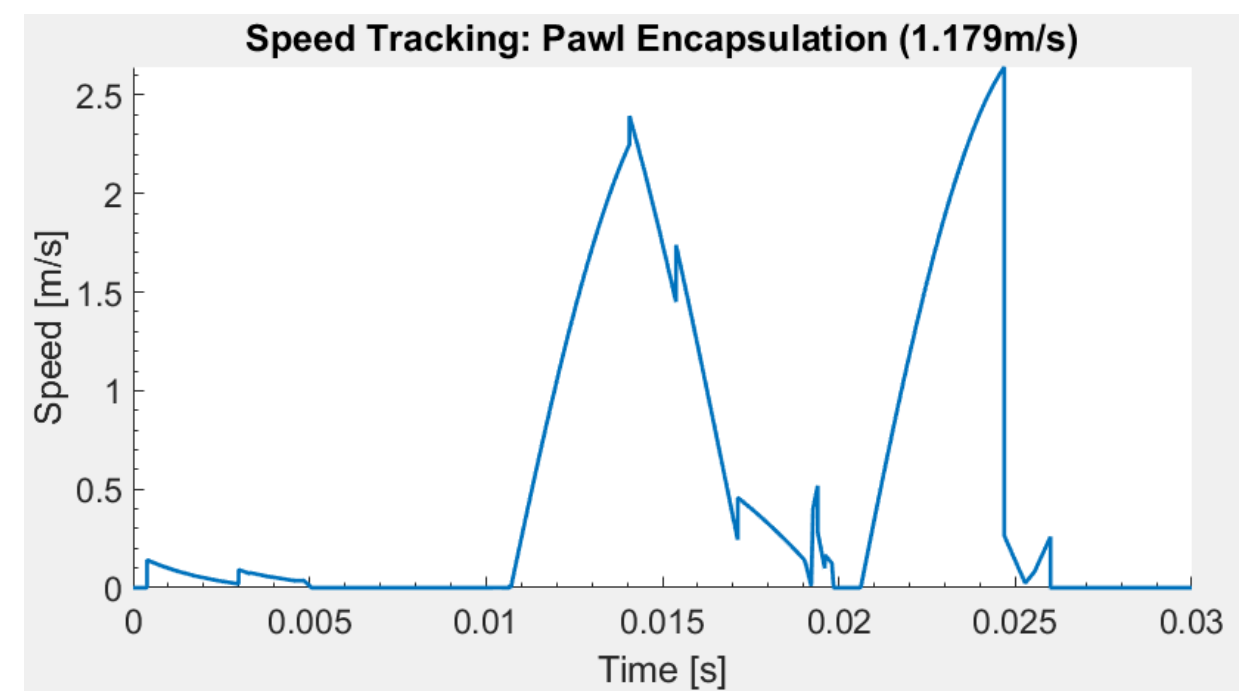

Figure 4.7: Tracked speed of the pawl encapsulation motion via a velocity probe. 
Table 4.2: Tangential impact speeds of the latch components.

\begin{tabular}{|c|c|c|}
\hline Impact Event & $\begin{array}{c}\text { Component Speed for } \\
\mathbf{0 . 8 5 1 m} / \mathbf{s}[\mathbf{m} / \mathbf{s}]\end{array}$ & $\begin{array}{c}\text { Component Speed for } \\
\mathbf{1 . 1 7 9 m} / \mathbf{s}[\mathbf{m} / \mathbf{s}]\end{array}$ \\
\hline Pawl/Ratchet & 2.03 & 1.86 \\
\hline Striker/Over-Slam Bumper \#1 & 0.851 & 1.179 \\
\hline Ratchet/Housing & 0.59 & 1.17 \\
\hline Pawl/Over-Slam Bumper \#2 & 2.67 & 2.64 \\
\hline
\end{tabular}

It is evident from the plot that the impact event between the Pawl/Ratchet and the Pawl/Over-Slam Bumper \#2 are similar in magnitude for both impact speeds with a difference of $0.17 \mathrm{~m} / \mathrm{s}$ and $0.03 \mathrm{~m} / \mathrm{s}$ respectively. However, the most notable change in speed was observed between the Striker/Over-Slam Bumper \#1 impact and the Ratchet/Housing impact with a difference of $0.328 \mathrm{~m} / \mathrm{s}$ and $0.58 \mathrm{~m} / \mathrm{s}$ respectively. Speed results from the RBD analysis were used as initial conditions for the ANSYS Explicit Dynamics computational model explained in the following section. For reference, a flow chart is presented in Appendix G outlining the input and output conditions of each computational module in addition to assumptions and simplifications.

\subsection{Explicit Dynamics}

The results generated by the RBD analysis only provided the tangential speed of each component just before impact. ANSYS Explicit Dynamics (ED) was able to determine the resulting forces experienced by the impacting parts via numerical methods. In an effort to reduce computational time, each impact event stated in Table 4.2 was modeled separately as opposed to all at once.

Prior to the analysis, it was assumed that the latch components were isotropic. Additionally, Magna Closures Inc. confirmed the latch components do not experience any plastic deformation during normal operation. Plastic deformation during operation would result in a failed latch. As a result, all latch 
components, including the thermoplastic vulcanizate, were modeled as linearly elastic materials as the forces experienced by the components were below the proportionality limit of each material.

\subsubsection{Model Assembly}

For each simulation pictured in Figures 4.8-4.11, the impacting bodies were situated approximately 7.5E-5m away from each other to replicate the moment immediately prior to contact. The main purpose was to reduce computational time as much as possible for each analysis. For every simulation, the first component mentioned in each impacting pair was free to move in the simulation space whereas the last component in each pair was fixed in space. As previously mentioned, the RBD speed results were used as initial conditions for each ED analysis. Further observation of the images shown in Figures 4.8-4.11 show segmented portions of the latch housing and over-slam bumpers. This was done to reduce the computational time required to successfully solve the simulation. The AUTODYN Solver was used to perform the reaction force analysis. Contact detection between the two impacting bodies was defined using Trajectory with a Penalty formulation. This is the most common setting for an analysis of this type [45].

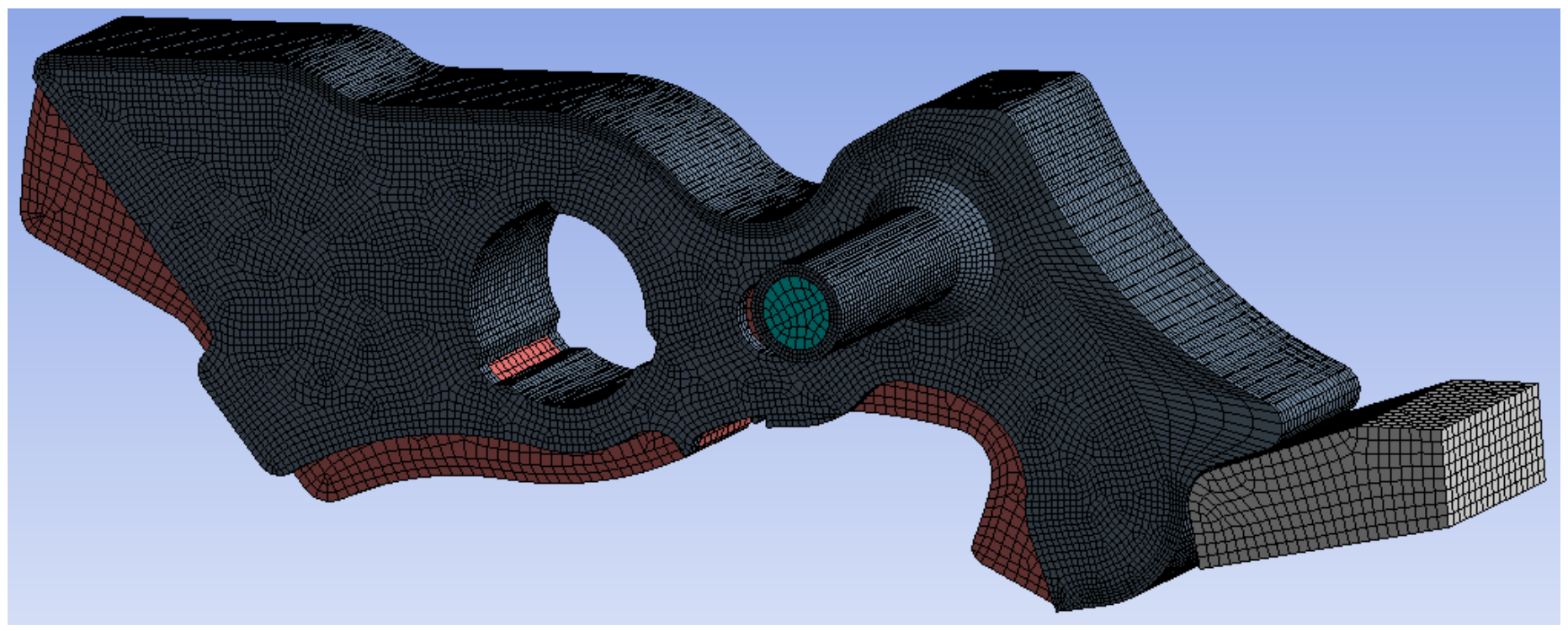

Figure 4.8: Discretized pawl and over-slam bumper \#2. 


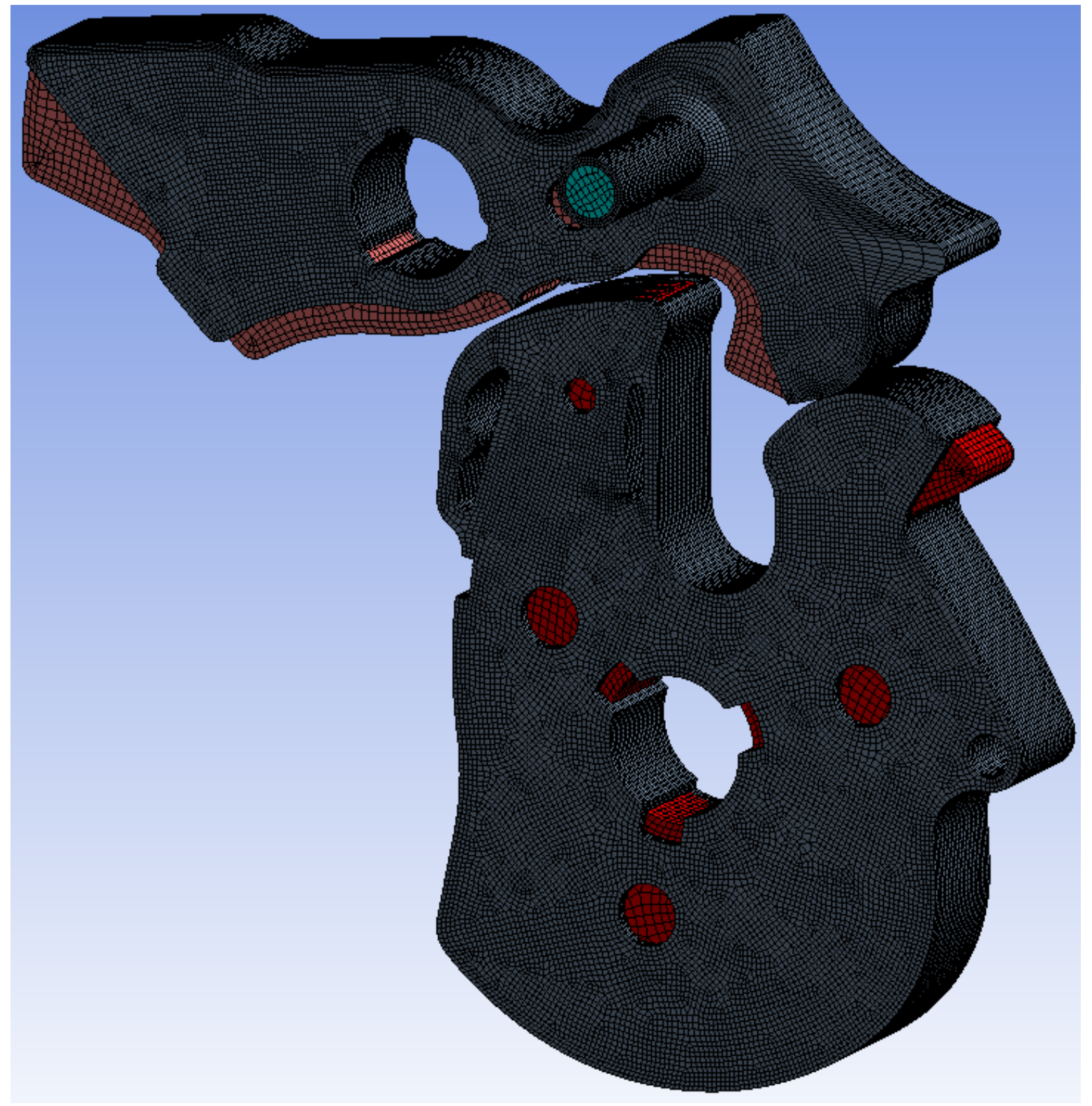

Figure 4.9: Discretized pawl and ratchet. 


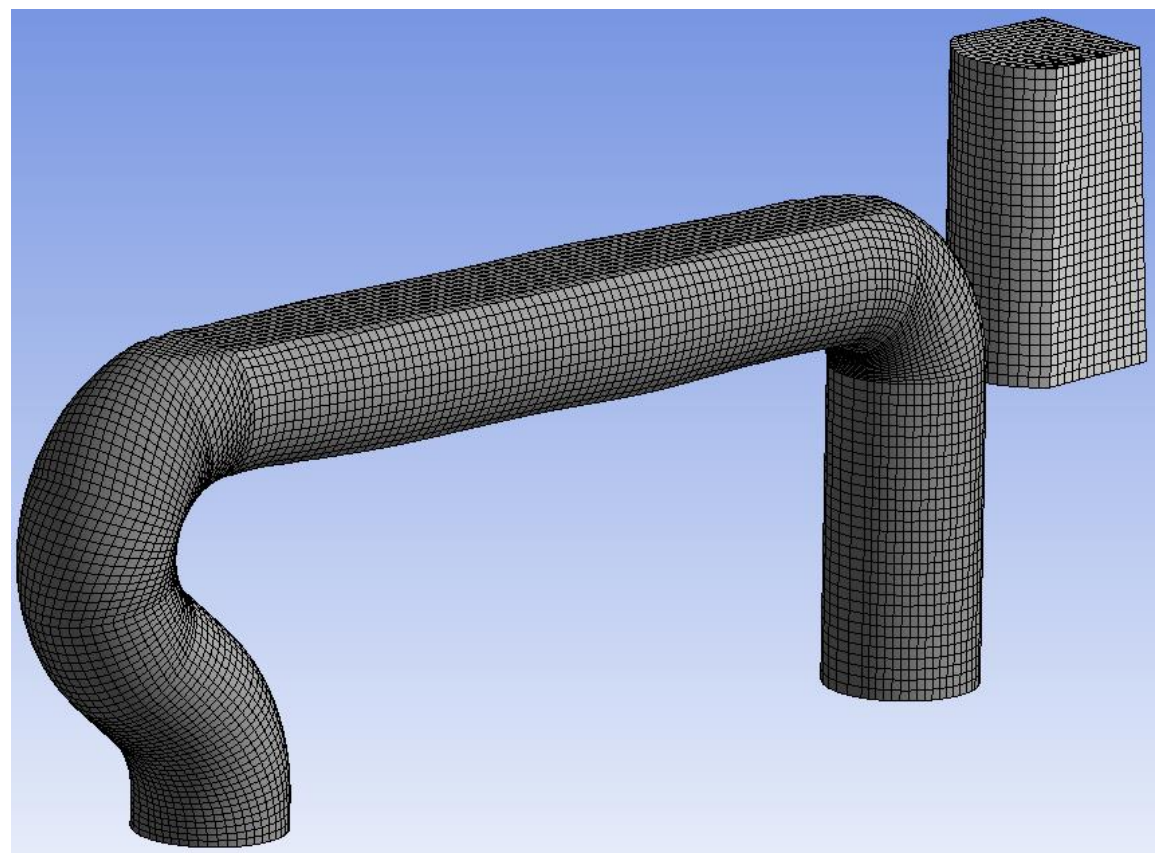

Figure 4.10: Discretized striker and over-slam bumper \#1.

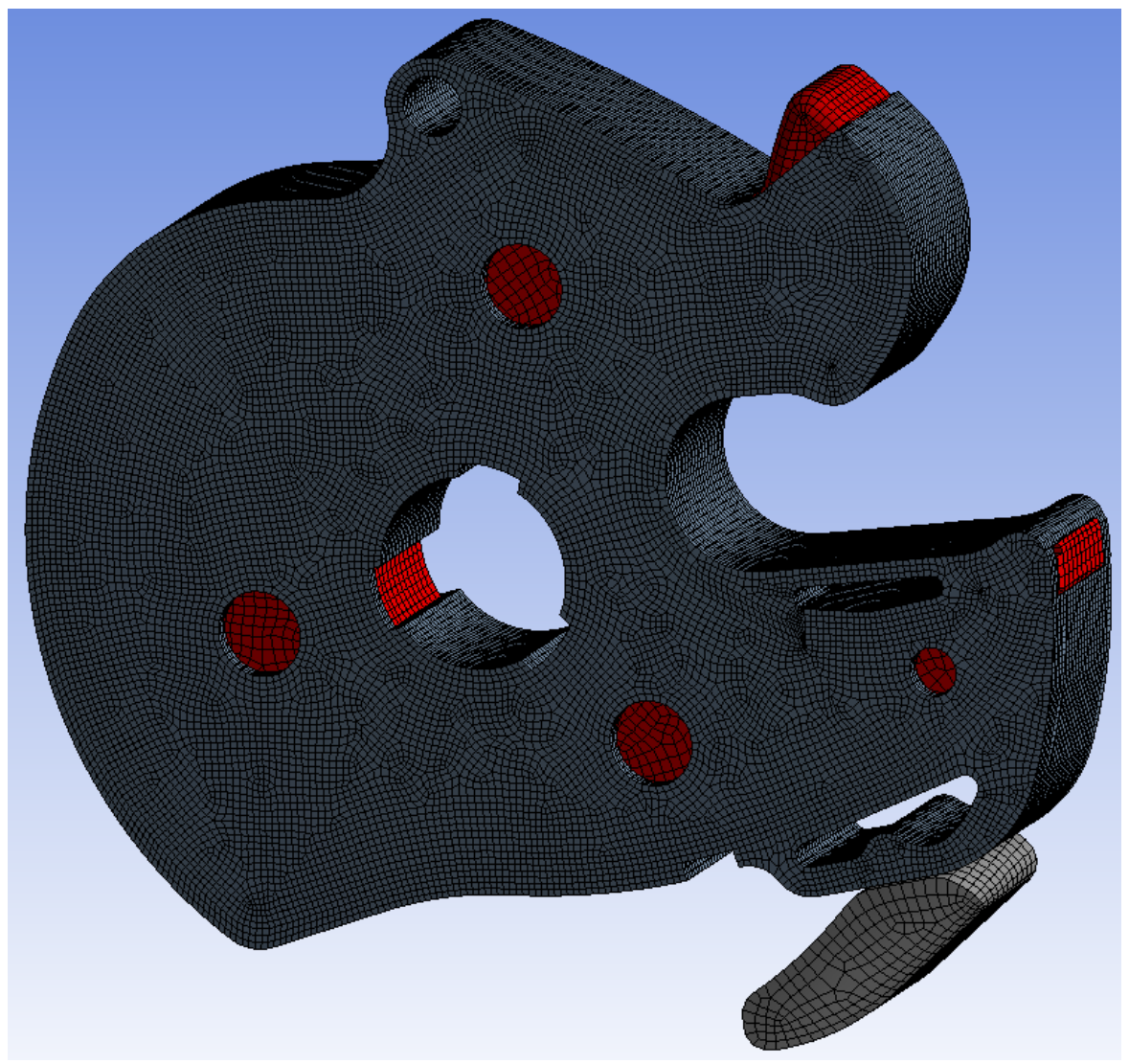

Figure 4.11: Discretized ratchet and latch housing. 
An important parameter in each ED simulation was the stiffness penalty function. This stiffness relationship between the contact body and target body must be established for contact to occur. Equation 4.1 was used to model this phenomenon

$$
F_{c}=k_{C S} \delta
$$

where $F_{c}$ is the contact force, $k_{C S}$ is the contact stiffness, and $\delta$ is the degree of penetration of the two impacting bodies [45]. The amount of penetration is dependent on the stiffness value $k_{C S}$. ANSYS automatically determines the optimal contact stiffness value for the model using Equation 4.2

$$
k_{C S}=\frac{(\beta)(A)^{2}(\mu)}{V_{o l}}
$$

where $k_{C S}$ is the contact stiffness, $\beta$ is the penalty factor ( 0.1 by default), $A$ is the area of contact segment, $\mu$ is the bulk modulus of the contact element, and $\mathrm{Vol}$ is the volume of the contact segment.

Equation 4.3 displays the expression used to determine the optimal time step

$$
\Delta t \leq S_{f}\left[\frac{E_{\min }}{C_{A}}\right]
$$

where $\Delta t$ is a stable time step, $S_{f}$ is the scale factor ( 0.9 by default), $E_{\min }$ is the smallest element dimension, and $C_{a}$ is the speed of stress wave [45]. The time step was internally calculated by the ANSYS artificial intelligence allowing for an accurate prediction of the highest frequency waves within the model. These waves were identified as stress and shock waves. This resulted in an average time step of 3.19E-9s for all the analyses investigated. 


\subsubsection{Mesh Generation}

The mesh skewness metric was used to verify the quality of each mesh prior to solving. Mesh skewness evaluates the angle between two conjoining lines of a quadrilateral shaped element and a triangular shaped element. Conjoining lines with an angle of $90^{\circ}$ for quadrilateral elements and $60^{\circ}$ for triangular elements are considered high quality. Each analysis achieved an average mesh skewness $<0.195$, which is considered "excellent" [46]. Each component used in the simulation was meshed using SOLID185 elements with an average element size of 3.0E-4m and an average time step of 3.19E-9s. Table 4.3 summarizes the average skewness, standard deviation, and number of elements and nodes of the meshed model used in each ED analysis.

Mesh convergence was not included in this study as the reliability of the mesh model was justified through other means. The minimum elements-per-wavelength (EPW) required to accurately model structural vibrations using linear shape elements is 12 EPW [11]. The average element size used in the models corresponds to 228.67 EPW which is well above the suggested value. The AUTODYN Solver restricts the use of higher-order SOLID186 hexahedral elements [45]. Therefore, SOLID185 hexahedral elements were used as opposed to SOLID185 tetrahedral elements which are known to be less accurate [11]. These factors, in addition to the skewness mesh metric discussed previously, were enough to justify the exclusion of a mesh convergence analysis for this particular study.

Table 4.3: Mesh metric and statistic results of the explicit dynamic analyses.

\begin{tabular}{|c|c|c|c|c|}
\hline Impact Event & $\begin{array}{c}\text { Average } \\
\text { Skewness }\end{array}$ & $\begin{array}{c}\text { Standard } \\
\text { Deviation }\end{array}$ & $\begin{array}{c}\text { Number of } \\
\text { Nodes }\end{array}$ & $\begin{array}{c}\text { Number of } \\
\text { Elements }\end{array}$ \\
\hline Pawl/Ratchet & 0.1371 & 0.1414 & 337,383 & 263,835 \\
\hline $\begin{array}{c}\text { Striker/Over-Slam } \\
\text { Bumper \#1 }\end{array}$ & 0.1923 & 0.1371 & 47,522 & 41,158 \\
\hline Ratchet/Housing & 0.1395 & 0.1298 & 205,968 & 160,732 \\
\hline $\begin{array}{c}\text { Pawl/Over-Slam } \\
\text { Bumper \#2 }\end{array}$ & 0.1404 & 0.1578 & 138,884 & 109,206 \\
\hline
\end{tabular}




\subsubsection{Results and Analysis}

The dynamic results were measured using a contact force probe affixed to each contact face. Figures 4.124.13 are sample plots of the force reaction as a function of time for the Striker/Over-Slam Bumper \#1 impact event. A Butterworth filter with a cut frequency of $500 \mathrm{~Hz}$ was applied to reduce the amount of noise present in the data [45]. A summary of the reaction forces from each impact event in sequential order are summarized in Table 4.4.

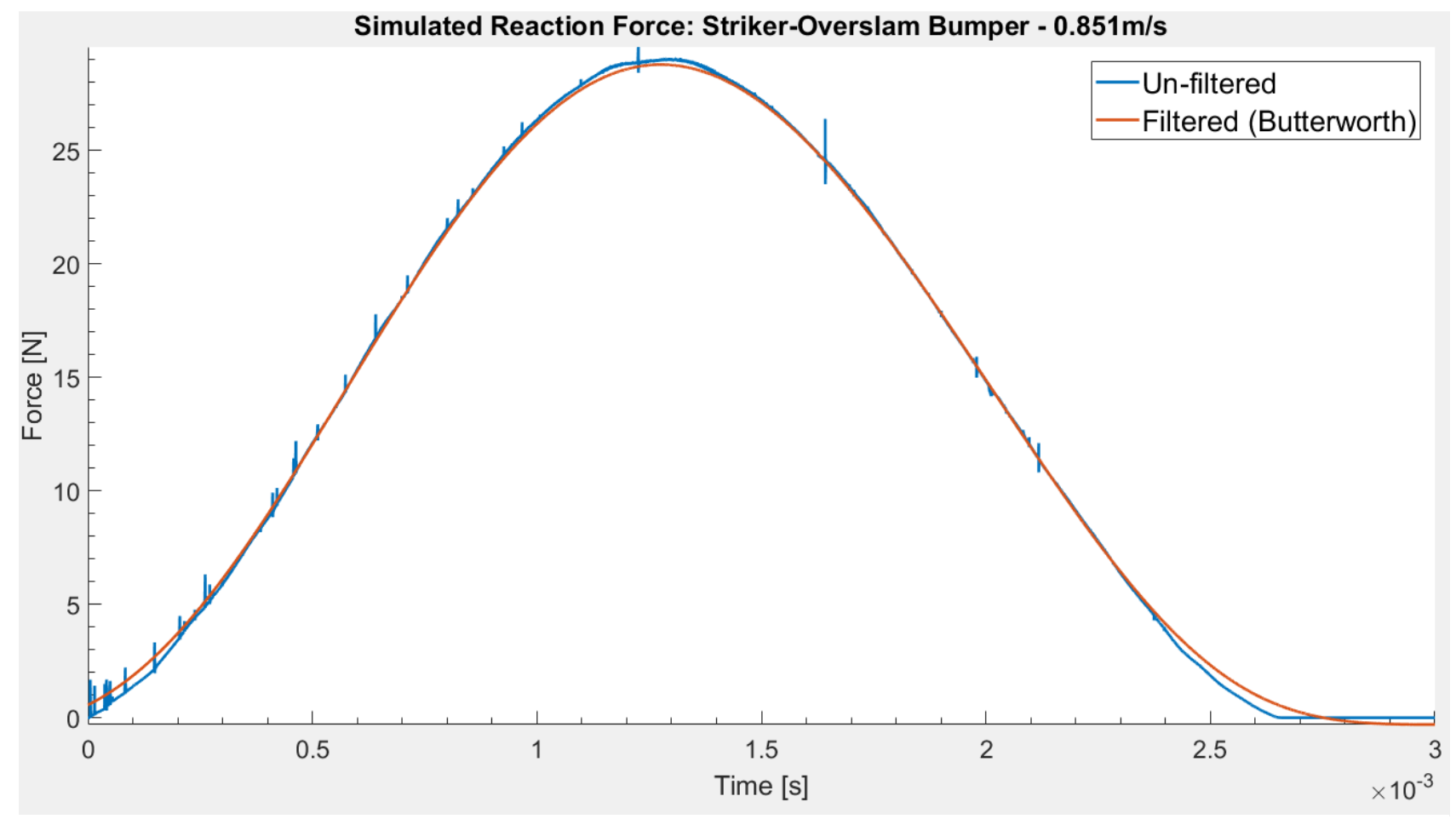

Figure 4.12: Reaction force results of the Striker/Over-Slam Bumper \#1 impact event at $0.851 \mathrm{~m} / \mathrm{s}$. 


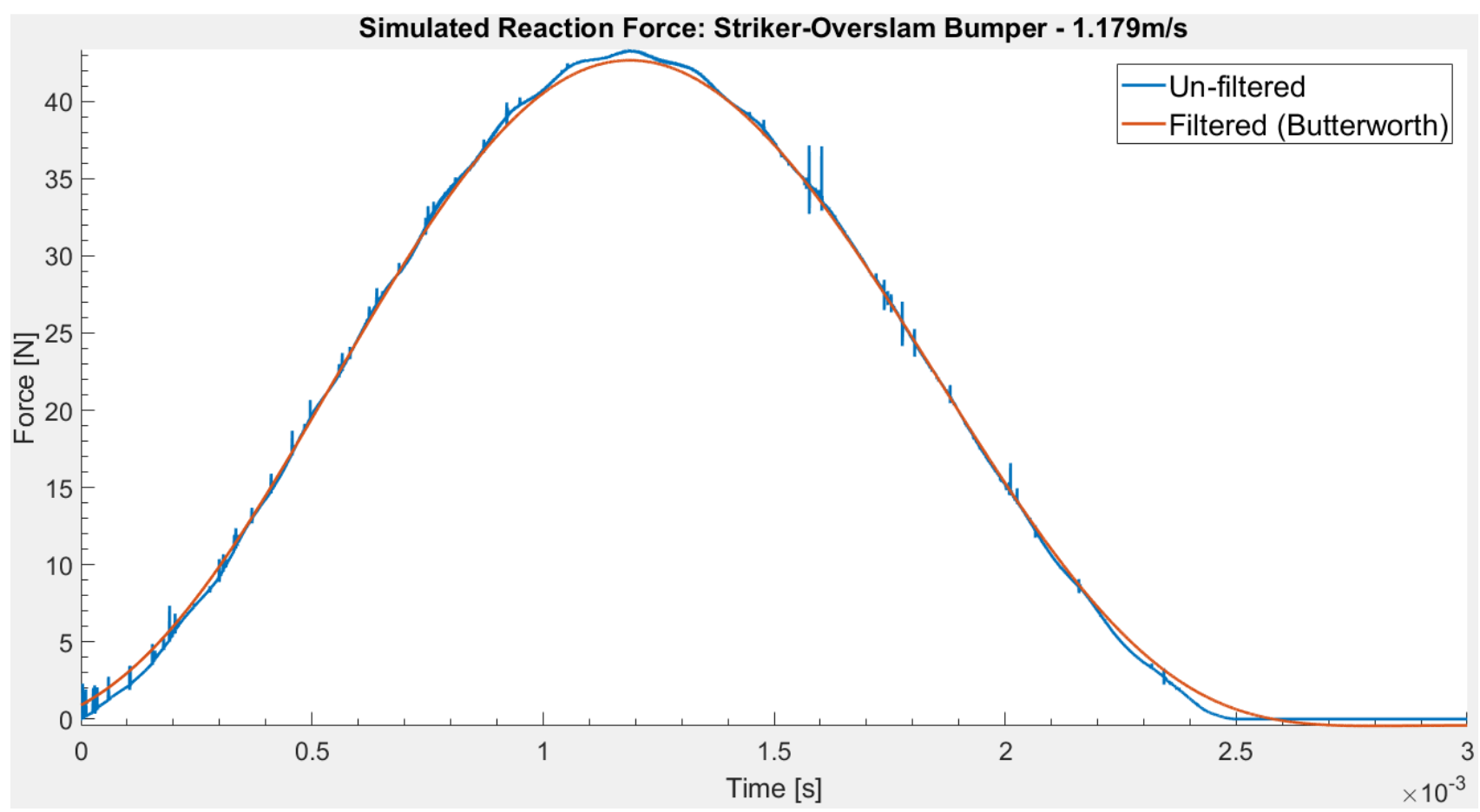

Figure 4.13: Reaction force results of the Striker/Over-Slam Bumper \#1 impact event at 1.179m/s.

Table 4.4: Reaction forces of the latch components.

\begin{tabular}{|c|c|c|}
\hline Impact Event & $\begin{array}{c}\text { Reaction Forces for } \\
\mathbf{0 . 8 5 1} \mathbf{m} / \mathbf{s}[\mathbf{N}]\end{array}$ & $\begin{array}{c}\text { Reaction Forces for } \\
\mathbf{1 . 1 7 9 m} / \mathbf{s}[\mathbf{N}]\end{array}$ \\
\hline Pawl/Ratchet & 2.40 & 2.26 \\
\hline Striker/Over-Slam Bumper \#1 & 28.77 & 42.66 \\
\hline Ratchet/Housing & 2.50 & 6.57 \\
\hline Pawl/Over-Slam Bumper \#2 & 12.40 & 12.25 \\
\hline
\end{tabular}

Analysis of the force reaction results indicate larger reaction forces between the Ratchet/Housing and Striker/Over-Slam Bumper resulting from an increase in closing speed. This suggests that these impact events have a greater influence on the acoustic response compared to the other impact events. Similarly, the results from the ED analysis were used as initial conditions for the acoustic Transient Structural computational model explained in the following section. 


\subsection{Transient Structural: Acoustic Simulation}

An effort was made to incorporate all the ED reaction force results in sequential order in one acoustic model. However, due to the inherent limitations of the workspace, only one impact event was investigated. As evidenced by the RBD and ED analyses, it is clear the dominant impact event during latch operation was the striker and over-slam bumper \#1. This impact event was the focus of the acoustic analysis. The formulation of the transient acoustic simulation in TS followed the research conducted by Cazzolato et al. in "Acoustic Analysis Using MATLAB and ANSYS" [11]. The following sections provide a brief overview of the acoustic modeling theory and best practices outlined by Cazzolato et al.

\subsubsection{Matrix Formulation}

According to Cazzolato, the finite element method for acoustic modeling recognizes the bi-directional coupling between a structure and the fluid it is in contact with. This is known as a fluid-structure interaction (FSI). For these types of analyses, "the equations relating to the structural dynamics need to be considered along with the mathematical description of the acoustics of the system, given by the Navier-Stokes equations of fluid momentum and the flow continuity equation" [11]. Simplified formulations of the fluid momentum and continuity equations helped shape the acoustic wave equation (Equation 3.1). The simplifications are governed by the following assumptions [11]:

- $\quad$ The acoustic pressure in the fluid medium is determined by the wave equation (Equation 3.1).

- $\quad$ The fluid is compressible where density changes are due to pressure variations.

- $\quad$ There is no mean flow of the fluid (initially static).

- $\quad$ The density and pressure of the fluid can vary along the elements and the acoustic pressure is defined as the pressure in excess of the mean pressure.

The stated assumptions allow the acoustic wave equation to determine the acoustic response of the fluid subject to any source causing a deviation from the mean pressure. Pressure-formulated acoustic elements are modeled by Equation 4.4. 


$$
p=\sum_{i=1}^{m} N_{i} p_{i}
$$

where $N_{i}$ is a set of shape functions, $p_{i}$ are acoustic nodal pressures at node $i$, and $m$ is the number of nodes forming the element. This is for a simple element with a finite number of nodes from which the overall pressure for the element is calculated. In pure acoustic analyses, the transient response is modelled using Equation 4.5.

$$
\left[M_{f}\right]\{\ddot{p}\}+\left[C_{f}\right]\{\dot{p}\}+\left[K_{f}\right]\{p\}=\left\{F_{f}\right\}
$$

where $\left[M_{f}\right]$ is the fluid mass matrix, $\left[K_{f}\right]$ is the fluid stiffness matrix, $\left[C_{f}\right]$ is the fluid damping matrix, $\left\{F_{f}\right\}$ is a vector of applied fluid loads, $\{p\}$ is a vector of unknown acoustic pressures and $\{\ddot{p}\}$ is a vector of the second derivative of acoustic pressure with respect to time.

The sound investigated in this study was generated by the D21MC latch, therefore a structural interaction must also be considered in the computational analysis. As a result, Equation 4.6 depicts the complex coupled matrix formulations for the vibrating structure and surrounding acoustic fluid.

$$
\left[\begin{array}{cc}
M_{s} & 0 \\
\rho_{0} R^{T} & M_{f}
\end{array}\right]\left\{\begin{array}{l}
\ddot{u} \\
\ddot{p}
\end{array}\right\}+\left[\begin{array}{cc}
C_{s} & 0 \\
0 & C_{f}
\end{array}\right]\left\{\begin{array}{l}
\dot{u} \\
\dot{p}
\end{array}\right\}+\left[\begin{array}{cc}
K_{s} & -R \\
0 & K_{f}
\end{array}\right]\left\{\begin{array}{l}
u \\
p
\end{array}\right\}=\left\{\begin{array}{l}
F_{s} \\
F_{f}
\end{array}\right\}
$$

where $\left[M_{S}\right]$ is the structural mass matrix, $\left[K_{S}\right]$ is the structural stiffness matrix, $\left[C_{S}\right]$ is the structural damping matrix, $\left[F_{S}\right]$ is a vector of applied structural loads, $\{u\}$ is a vector of unknown nodal displacements, $\{\dot{u}\}$ and $\{\ddot{u}\}$ are a vectors of the first and second derivative of displacement with respect to time, $\rho_{0}$ is fluid density, and $[R]$ is the coupling matrix that accounts for the effective surface area associated with each node on the fluid structure interface (FSI) [11] [47]. To clarify, the FSI allows for the transfer of energy from a vibrating structure to an acoustic domain in the form of sound waves. 
Application of the FSI is discussed in a subsequent section. It should be mentioned that an in-depth analysis of the complex matrix formulations was beyond the scope of this work.

Due to the unsymmetrical nature of Equation 4.6, ANSYS uses an "unsymmetric" algorithm to solve the formulation. This simplified analysis was performed in ideal conditions such that any effects due to the acoustical impedance of the fluid as well as the damping effects of the structure were not considered. Cazzolato mentions that employing a numerical damping factor can improve the stability of the transient numerical integration. Numerical damping is not a traditional form of damping as it is geared toward stabilizing the numerical solution process as opposed to adding damping values to the system's damping matrix seen in Equation 4.6. This is done to mitigate the numerical noise contributed by the higher natural frequencies of the system [48]. Therefore, the suggested numerical damping value of Gamma $=0.005$ was used. The term Gamma is denoted as the Amplitude Decay Factor.

\subsubsection{D21MC Acoustic Model Formulation}

The literature states the acoustic fluid surrounding the latch must possess a curved outer surface to correctly model the radial spread of the sound from the latch. With reference to the work performed by Von Estorff, a curved surface is the only surface type accepted by sound absorbing infinite elements as depicted by Figure 4.14. Infinite elements are required to satisfy the Sommerfeld radiation condition which states that outgoing acoustic waves from acoustic sources should continue to propagate outward to infinity [11] [42]. To clarify, no sound energy shall be reflected off the curved boundary of the acoustic enclosure with the possibility of being measured by the simulated receivers.

ANSYS recommends spherical bodies be used to model 3D geometry with the source situated at the centroid. The distance between the edge of the sound source and outer surface of the spherical body should be separated by at least 0.2 times the largest acoustic wavelength $\left(0.2 \lambda_{\max }\right)$ [11]. Chapter 3 defined the lower and upper bounds of the frequency band investigated as $160 \mathrm{~Hz}\left(f_{\min }\right)$ and $5 \mathrm{kHz}\left(f_{\max }\right)$. 
For the acoustic simulation these values were imperative in determining the correct size of the acoustic region in addition to the mesh density.

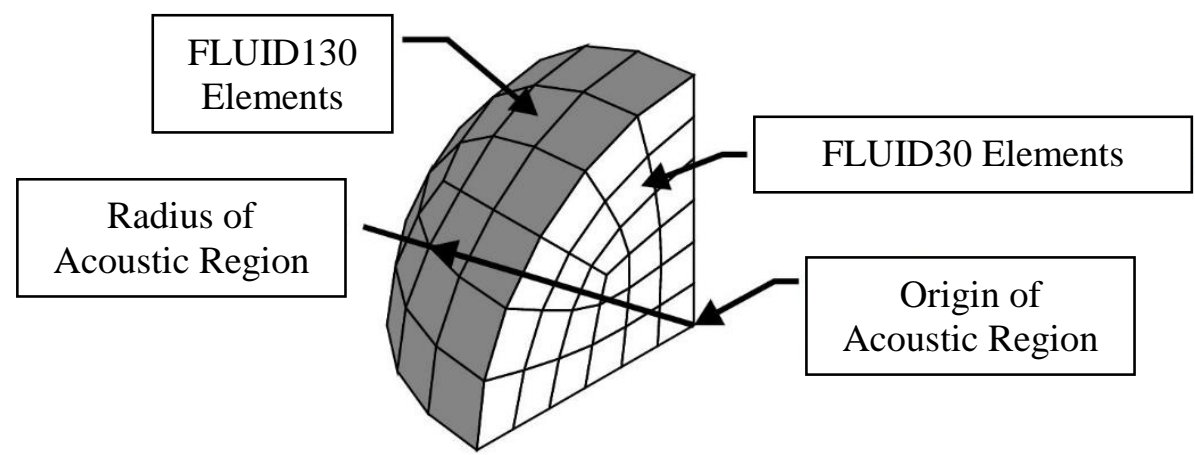

\section{Figure 4.14: Placement of fluid elements for modelling a free field environment.}

Equation 4.7 was used to determine the maximum wavelength

$$
\lambda_{\max }=\frac{c}{f_{\min }}
$$

where $\lambda_{\max }$ is the maximum wavelength investigated, $c$ is the speed of sound in air $(343 \mathrm{~m} / \mathrm{s})$ and $f_{\min }$ is the lower investigated frequency limit. This equated to a value of $\lambda_{\max }=2.14 \mathrm{~m}$. The resulting minimum distance away from the vibrating structure was determined to be $r_{\min }=0.428 \mathrm{~m}$. Using the dimensions of the latch mentioned in Figure 4.2, the equivalent radius of the latch was determined following Equation 4.8.

$$
r_{\text {latch }}=\sqrt{\left(\frac{a}{2}\right)^{2}+\left(\frac{b}{2}\right)^{2}}
$$

where $r_{\text {latch }}$ is the equivalent radius of the latch, and $a$ and $b$ are the longest dimensions of the latch. This equated to a value of $r_{\text {latch }}=0.0971 \mathrm{~m}$. Summing $r_{\text {latch }}$ and $r_{\text {min }}$ results in a suggested acoustic region radius of $R_{\text {acoustic }}=0.526 \mathrm{~m}$ which was rounded to $R_{\text {acoustic }}=0.53 \mathrm{~m}$ for convivence. Given the fact 
that microphone placement used in the experimental procedure was $0.5 \mathrm{~m}$ normal to the latch, the curved outer surface of the acoustic region would extend past the simulated receivers by approximately $0.03 \mathrm{~m}$. This buffer region between the receivers and acoustic boundary ensures that the simulated receivers were completely surrounded by acoustic media, which is recommended in the literature [11].

As stated in the literature, an ideal acoustic region is a solid sphere, hemisphere or a quarter-sphere. However, the generation of an acoustic region of this size was not possible as ANSYS would continuously fail part-way through the solution. Previous attempts at generating a suitable region can be seen in Figure 4.15.
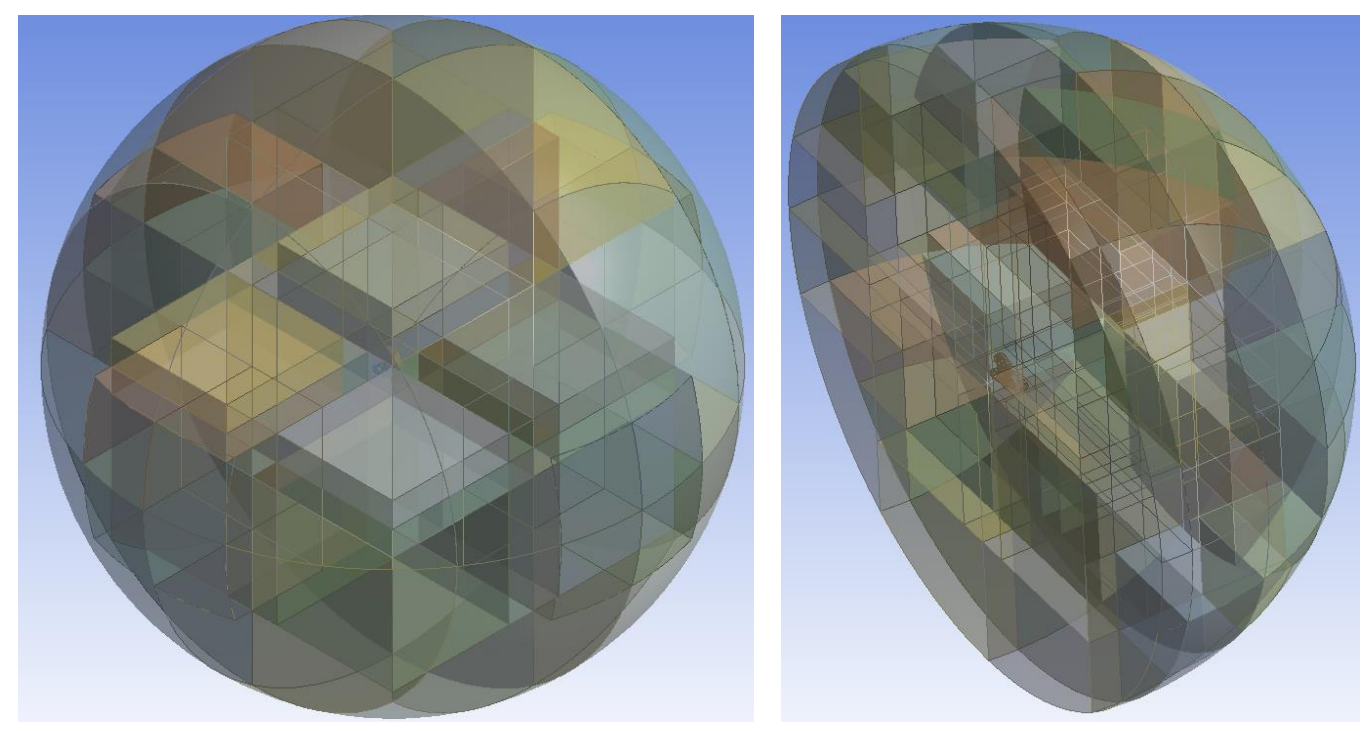

Figure 4.15: Inadequate acoustic regions resulting from the inherent limitations of the workstation.

It was concluded that the cause of the failure was that ANSYS would run out of available hard-disc space.

This was due to the extremely large acoustic matrix generated within ANSYS to solve the model.

Therefore, it was confirmed that hard-disc space was the primary limitation which dictated the size and complexity of the acoustic simulation. As a result, a 'slivered' acoustic region with a curved outer face was chosen. The computational procedure benefitted from the slivered geometry as it was simpler to 
discretize with an accurate mesh; it was able to model the direct acoustic energy from the impact; infinite elements could still be applied to the curved boundary; and the workstation was able meet the computational demand required to solve the model. The only disadvantage was that the three microphone receivers seen in the experimental procedure were separated in the simulated environment, resulting in six acoustic simulations instead of two. The slivered acoustic regions are depicted in Figure 4.16-4.19. As with the latch components, the acoustic region was segmented into twelve regions. This procedure is discussed further in a subsequent section.

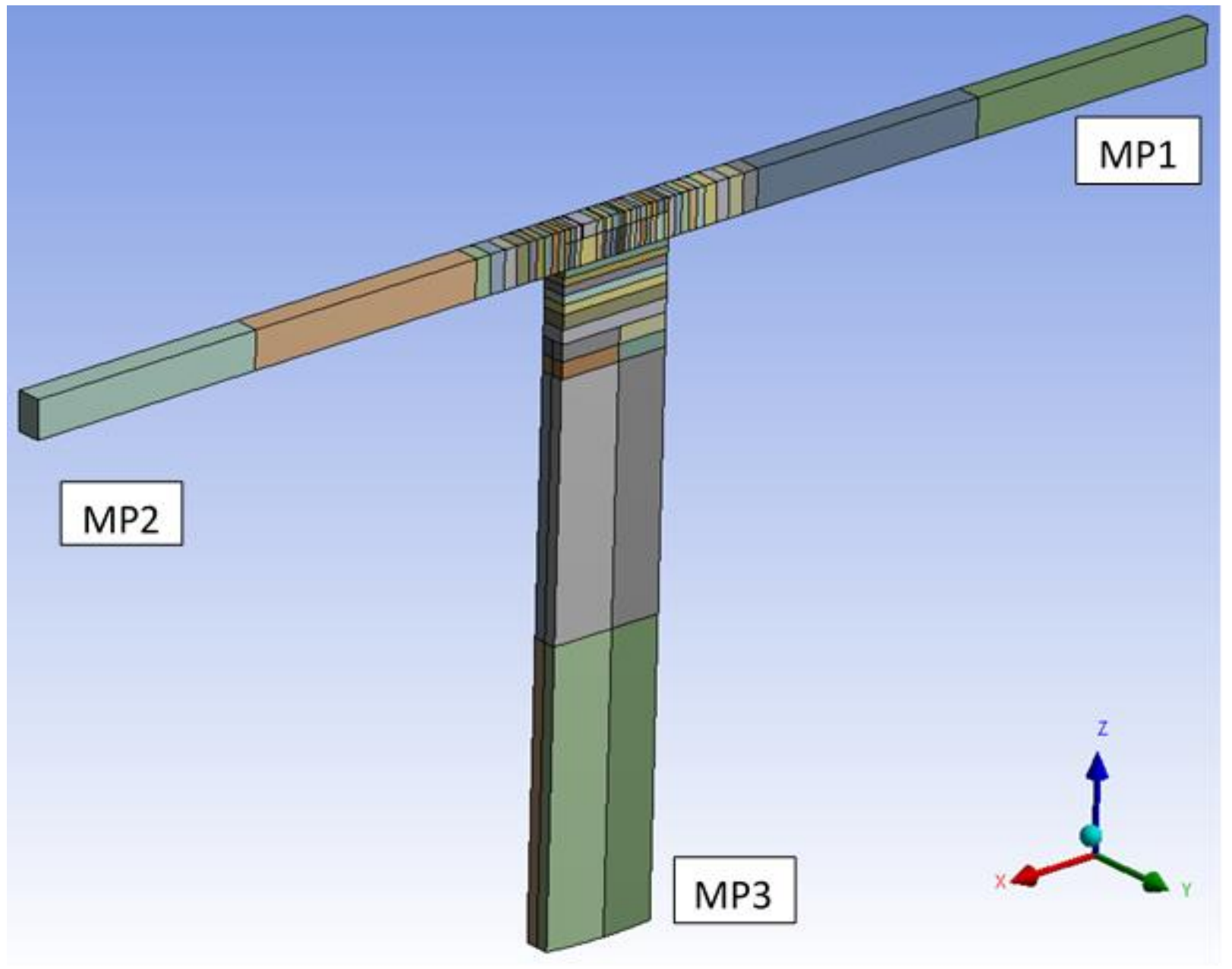

Figure 4.16: Slivered acoustic region geometry used for TS simulations. 

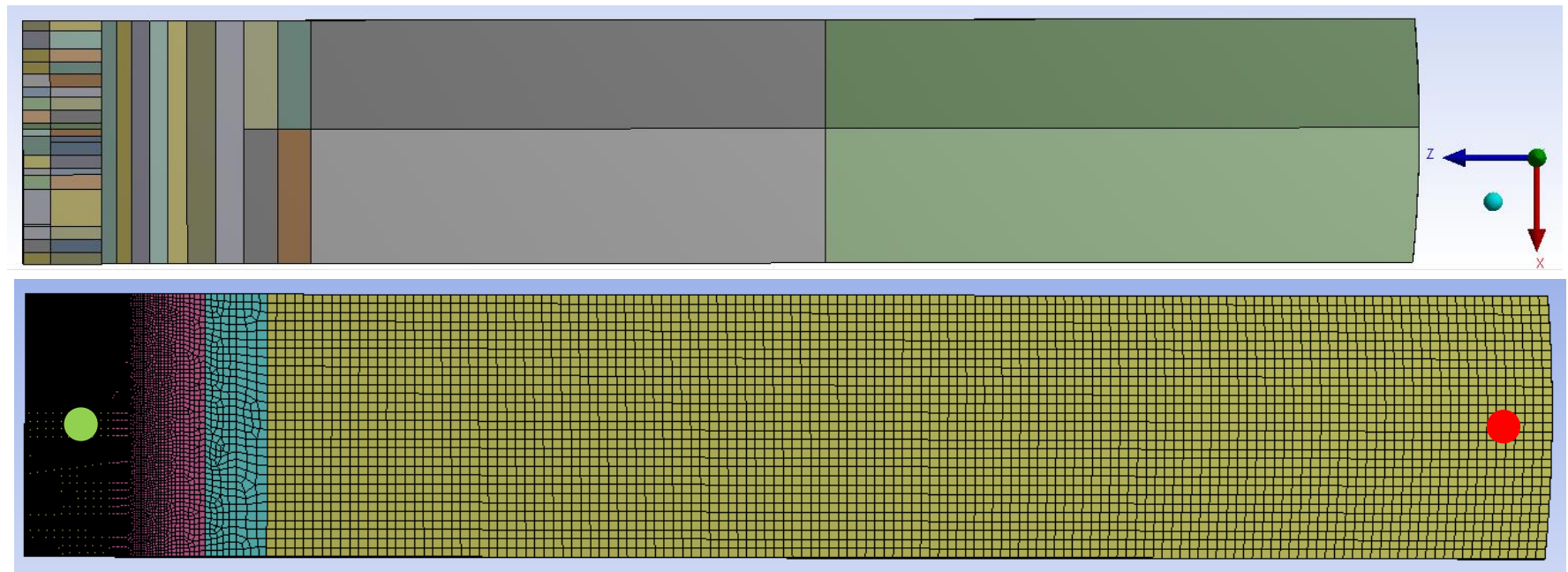

Figure 4.17: Meshed (Bottom) and not meshed (Top) MP3 acoustic region. Colours were used to identify bodies for mesh generation. Virtual microphone located $0.5 \mathrm{~m}$ from the latch center represented by the red marker. The latch center is represented by the green marker and is common for all acoustic simulations.

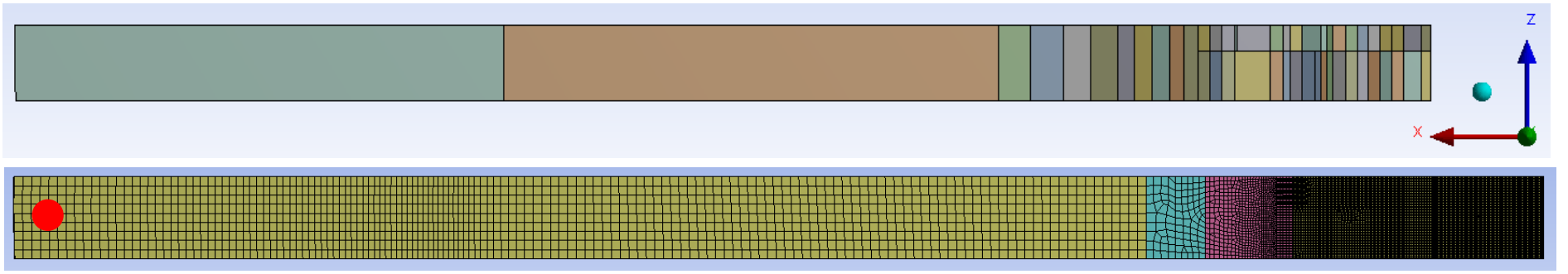

Figure 4.18: Meshed (Bottom) and not meshed (Top) MP2 acoustic region. Virtual microphone located 0.5m from the latch center represented by the red marker.

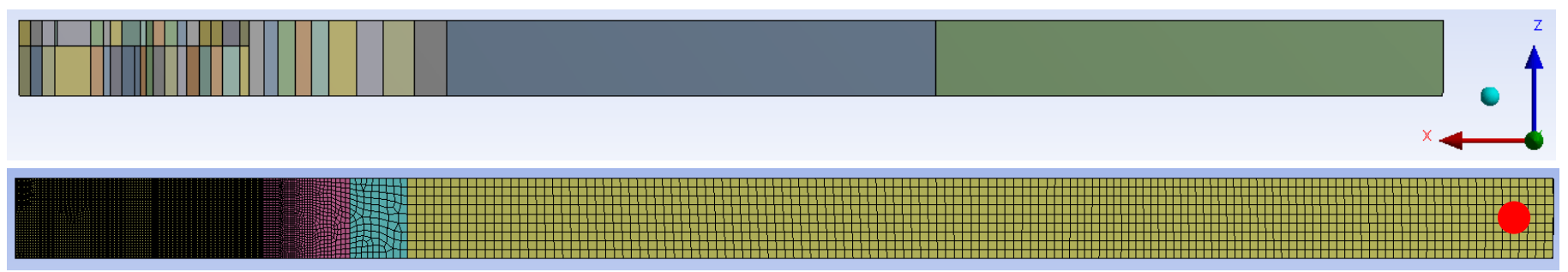

Figure 4.19: Meshed (Bottom) and not meshed (Top) MP1 acoustic region. Virtual microphone located 0.5m from the latch center represented by the red marker. 


\subsubsection{Element Types and Shape Function}

The limitations mentioned in the previous section also forced the acoustic region to be formulated using linear shape elements as opposed to quadratic shape elements; quadratic elements are known to be more accurate. To compensate for this, ANSYS requires a minimum of 12 linear elements per wavelength (EPW) to accurately model the structural and acoustic response up to the frequency of interest. A maximum frequency of $5 \mathrm{kHz}$ was applied in Equation 4.9 .

$$
S_{E}=\left(\frac{c}{f_{\max }}\right)\left(\frac{1}{E P W}\right)
$$

where $S_{E}$ is the element size, $c$ is the speed of sound in air, $f_{\text {max }}$ is the upper investigated frequency limit, and EPW is the number of elements per wavelength. This equated to a maximum element size of $5.72 \mathrm{E}-3 \mathrm{~m}$. This value was used as a guide as the model was discretized using smaller element sizes for improved accuracy.

Five element types were utilized to discretize the structural striker and over-slam bumper as well as the acoustic region. These elements were SOLID185, CONTA174, TARGE170, FLUID30 and FLUID130. Table 4.5 outlines the element type associated with a specific body or acoustic region.

Table 4.5: Element types used to mesh each body.

\begin{tabular}{|c|c|}
\hline Element Type & Simulation Body \\
\hline SOLID185 & Striker, Over-Slam Bumper \#1 \\
\hline CONTA174 & Striker (surface), Over-Slam Bumper \#1 (surface) \\
\hline TARGE170 & Acoustic surface adjacent to the components \\
\hline FLUID30 & Acoustic Region \\
\hline $\begin{array}{c}\text { FLUID130 (Infinite } \\
\text { element) }\end{array}$ & Acoustic Region (curved outer surface) \\
\hline
\end{tabular}




\subsubsection{Model Discretization}

Discretization of the structural and acoustic bodies was performed in ANSYS Workbench. An overly coarse mesh was avoided to prevent the model from becoming "artificially stiff", resulting in poor energy transfer from one region of the model to the other [11]. Conversely, an overly fine mesh was not suitable as it would be computationally inefficient. Proper care was exercised to create a discretized acoustic model as shown in Figures 4.17-4.19. A close-up of the MP3 region split along the x-z plane (Figure 4.20) exhibits a gradual transition in mesh density from a small element size at the latch components to a larger element size towards the outer region of the acoustic domain. Table 4.6 demonstrates the element size gradient and the respective EPW that was followed by each simulation allowing for more justifiable comparisons. The largest element size used was $3.45 \mathrm{E}-3 \mathrm{~m}$ which was approximately 1.65 times larger than the minimum required EPW. Gradually increasing the size of the elements was done to shorten the time required to solve the model. If workstation limitations were not a concern, an ideal discretized model would exhibit a single global element size throughout. It was concluded that the discretization method employed was well-suited for this analysis and it was deemed appropriate to move forward simulation procedure.

Table 4.6: Element mesh size of acoustic regions.

\begin{tabular}{|c|c|c|c|c|c|c|c|c|c|c|c|c|}
\hline $\begin{array}{c}\text { Acoustic } \\
\text { Division }\end{array}$ & $\begin{array}{c}\text { Latch } \\
\text { Region }\end{array}$ & $\mathbf{1}$ & $\mathbf{2}$ & $\mathbf{3}$ & $\mathbf{4}$ & $\mathbf{5}$ & $\mathbf{6}$ & $\mathbf{7}$ & $\mathbf{8}$ & $\mathbf{9}$ & $\mathbf{1 0}$ & $\mathbf{1 1}$ \\
\hline $\begin{array}{c}\text { Element } \\
\text { Size [m] }\end{array}$ & $5.00 \mathrm{E}-4$ & $6.00 \mathrm{E}-4$ & $7.50 \mathrm{E}-4$ & $1.05 \mathrm{E}-3$ & $1.50 \mathrm{E}-3$ & $2.00 \mathrm{E}-3$ & $2.70 \mathrm{E}-3$ & $3.45 \mathrm{E}-3$ & $3.45 \mathrm{E}-3$ & $3.45 \mathrm{E}-3$ & $3.45 \mathrm{E}-3$ & $3.45 \mathrm{E}-3$ \\
\hline EPW & 137.20 & 114.30 & 91.47 & 65.33 & 45.73 & 34.30 & 25.41 & 19.88 & 19.88 & 19.88 & 19.88 & 19.88 \\
\hline
\end{tabular}




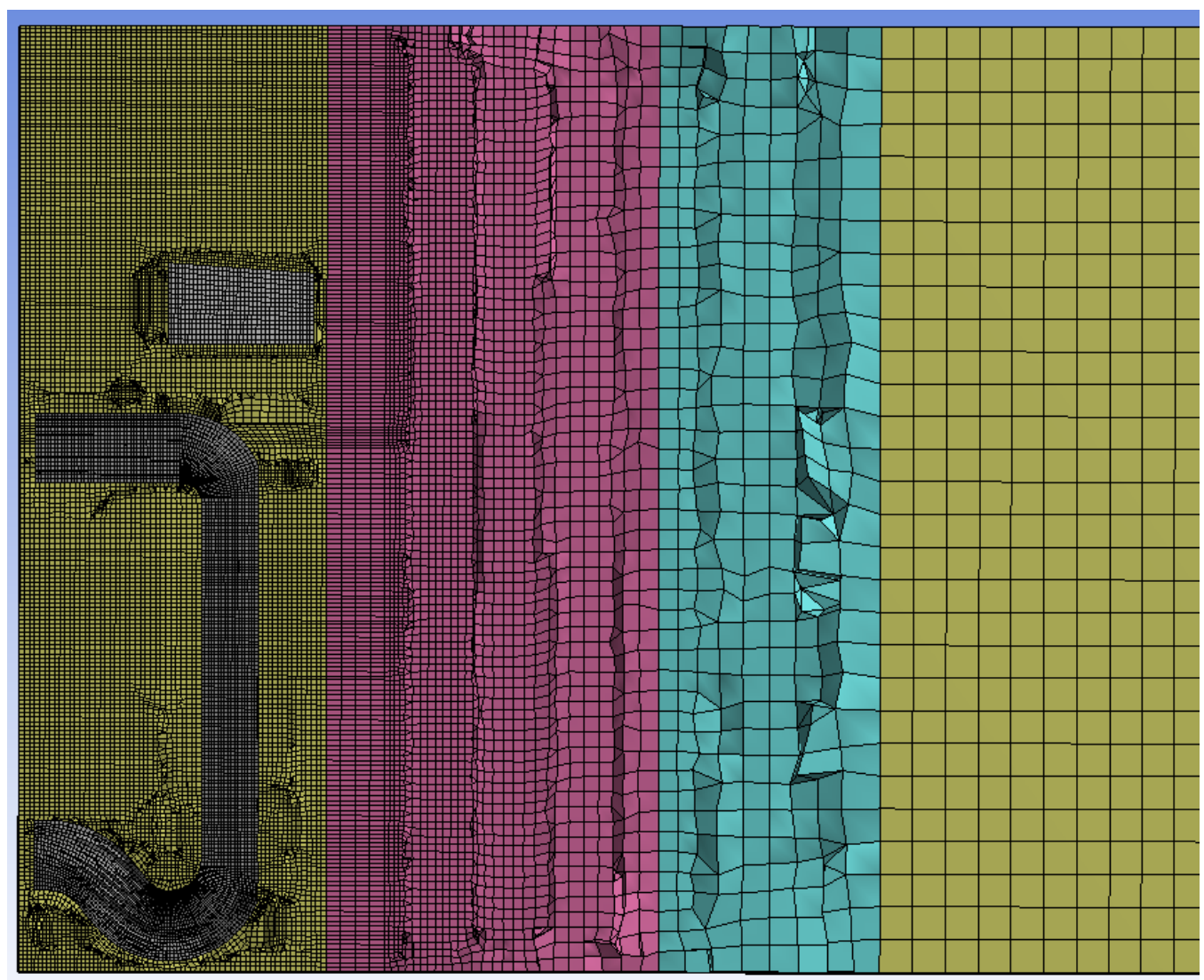

Figure 4.20: depiction of the gradual mesh density transition from the latch center towards the outer boundary of the acoustic region.

As with the ED analysis, mesh convergence was not included as the reliability of the mesh was justified by EPW, element type and skewness. With the inclusion of transition regions from a finer mesh density to a coarser mesh density, all variations of the SOLID185 element such as hexahedral, wedge, pyramid and tetrahedral elements were required. However, the quantity of tetrahedral elements was regulated through slow transition from one density region to the other. If this was not regulated, sudden changes in density would result in a considerable increase in tetrahedral elements. Much like the ED analysis, each acoustic analysis achieved an average mesh skewness $<0.160$, which is considered "excellent" [46]. Table 4.7 summarizes the average skewness, standard deviation, and number of elements and nodes of the meshed 
model used in each TS analysis. These factors were enough to justify the exclusion of a mesh convergence analysis for this particular study.

Table 4.7: Mesh metric and statistic results of the transient structural acoustic analyses.

\begin{tabular}{|c|c|c|c|c|}
\hline $\begin{array}{c}\text { Transient } \\
\text { Structural } \\
\text { Acoustic Model }\end{array}$ & $\begin{array}{c}\text { Average } \\
\text { Skewness }\end{array}$ & $\begin{array}{c}\text { Standard } \\
\text { Deviation }\end{array}$ & $\begin{array}{c}\text { Number } \\
\text { of Nodes }\end{array}$ & $\begin{array}{c}\text { Number of } \\
\text { Elements }\end{array}$ \\
\hline MP1 & 0.1596 & 0.2281 & 813,514 & 771,496 \\
\hline MP2 & 0.1596 & 0.2281 & 813,514 & 781,430 \\
\hline MP3 & 0.1604 & 0.2300 & 863,799 & 893,510 \\
\hline
\end{tabular}

\subsubsection{Fluid Structure Interaction}

Recalling the matrix formulation described in a previous section, $[R]$ represents the coupling matrix between the structural and acoustical matrices. In practice, $[R]$ is included in the solution when FSI is enabled. Figure 4.21 depicts a simplified FSI interface between SOLID185 and FLUID30 elements. The structural elements represent the striker and bumper with only displacement degrees of freedom (DoF) enabled. The hashed area represents the acoustic elements with pressure and displacement DoF's enabled. The solid line represents the FSI condition coupling the solid structure with the fluid medium. This thin layer of acoustic elements facilitates the bi-directional coupling between the vibrating structure and the acoustic pressure within the fluid. Acoustic elements connected to a fluid-structure interface are the only acoustic elements able to move within the model. The remaining acoustic elements shown in white only have pressure DoFs enabled to measure the radiating acoustic pressure [11]. 


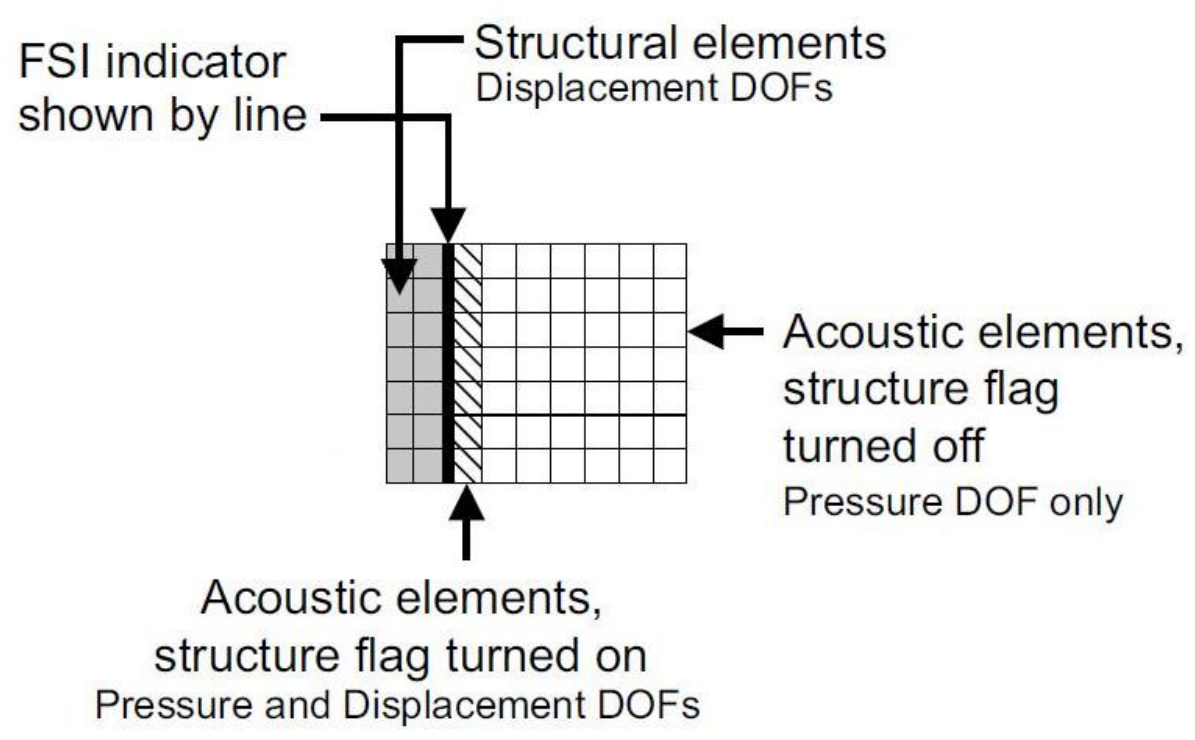

Figure 4.21: Fluid structure interaction between structural and acoustic elements.

With regard to mesh formulation near the FSI, Cazzolato advised,

...that the mesh density of the acoustic elements in the region of fluidstructure interaction should have a similar mesh density, and ideally coincident nodes, as the structural elements, so that the displacement of the nodes belonging to the structural elements will cause an identical displacement of the nodes belonging to the acoustic elements. The mesh density of the acoustic elements farther away from the fluid-structure interaction region need only have sufficient mesh density for the acoustic wavelength being investigated. [11]

In keeping with this statement, both the striker and bumper were discretized using 5E-4m sized elements in an effort to obtain a coincident node condition between the structural and acoustic bodies. However, due to the highly curved striker geometry, this condition was difficult to achieve as seen in Figure 4.22. 


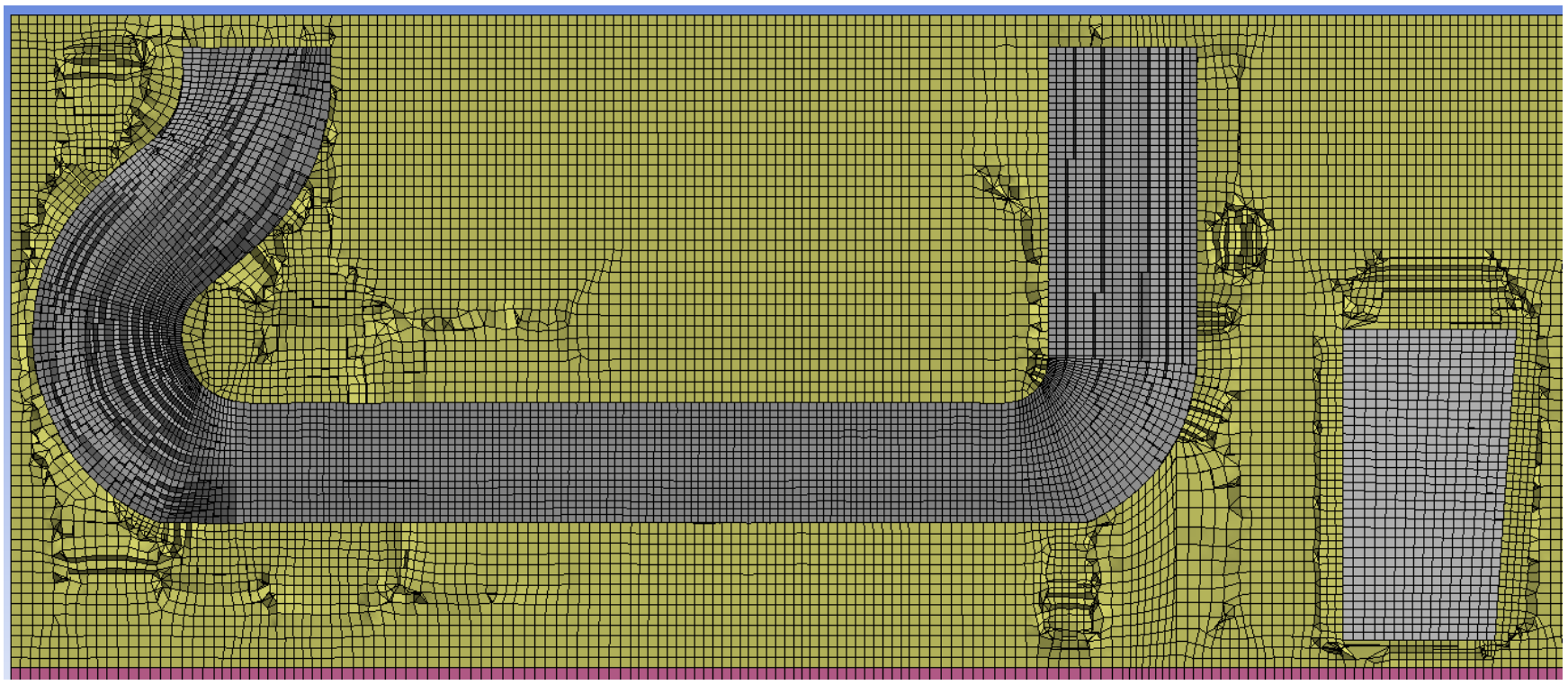

Figure 4.22: Discretized latch components (grey and white) and acoustic region (yellow) with FSI enabled.

\subsubsection{Simulation Procedure}

The same acoustic simulation procedure was followed for each microphone position. For this reason, only the MP3 transient acoustic analysis is presented. To facilitate the transfer of vibrational energy from the striker and over-slam bumper, a contact region must be defined. To recall, the FSI condition enables the acoustic elements adjacent to the structure to model the structural displacements in addition to measure the sound pressure.

The contact condition used was a "bonded" type with "symmetric" behavior (default). All other settings were set to "program controlled". To use this setting correctly, at least one surface must be defined as the "Contact" surface and another as a "Target" surface. This is done automatically or manually within the ANSYS environment. The "bonded" setting ensured no sliding or separation between faces or edges was allowed [49]. The "symmetric" behavior setting signifies that the Contact surfaces are constrained from penetrating the Target surface and vice versa [49]. In each simulation, the outer surface of the striker and bumper was defined as the Contact surface and the acoustic surface adjacent to the components was 
defined as the Target surface. This condition is outlined in Figure 4.23. The striker and bumper were fixed in space as shown in Figure 4.24. Caution was exercised to prevent over-constraining the model.

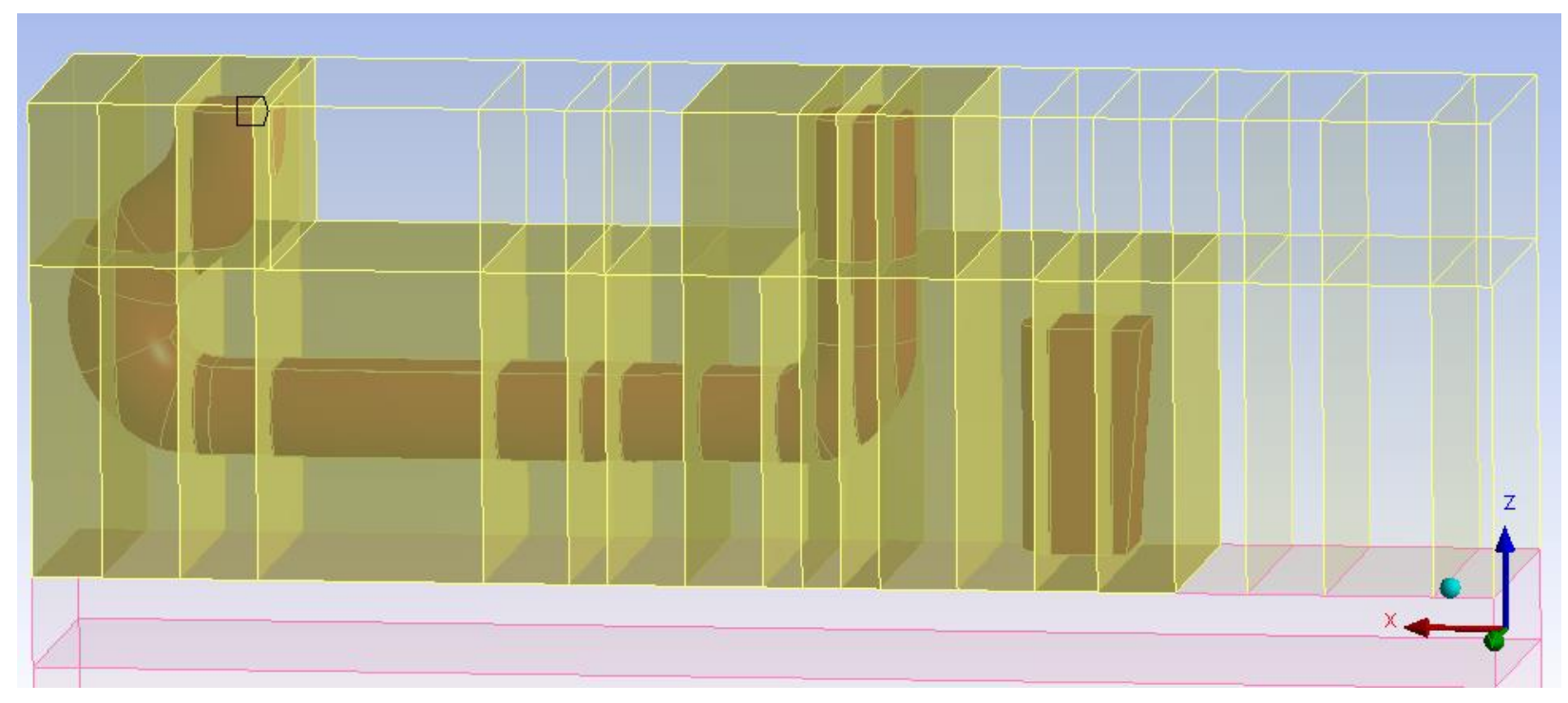

Figure 4.23: Latch body defined as Contact surface shown in red and the acoustic Target surface shown in yellow.

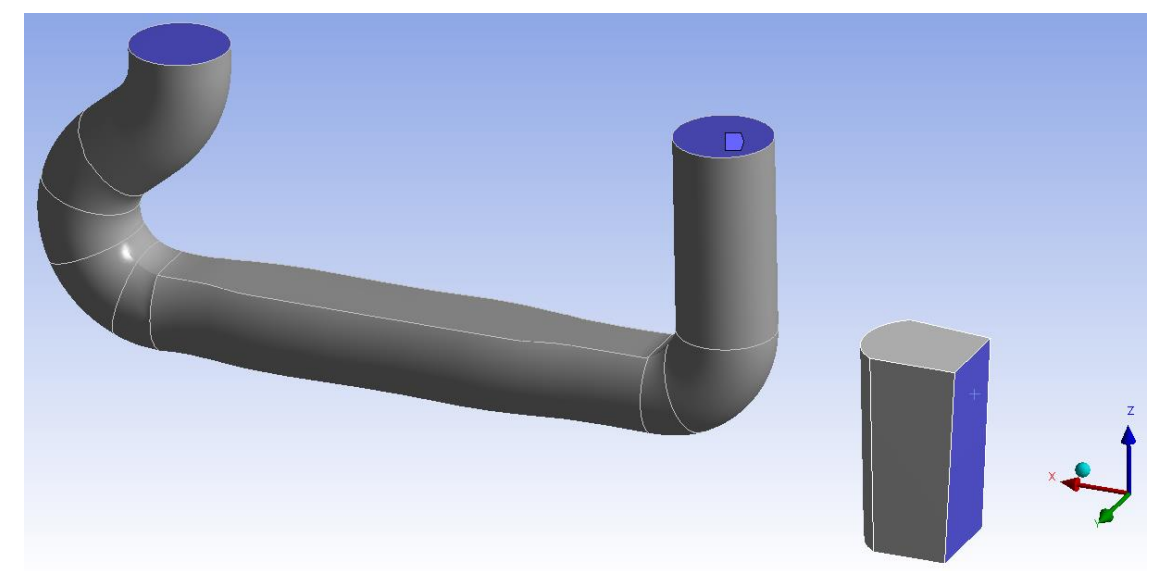

Figure 4.24: Fixed boundary condition shown in blue.

As with the experimental tests, each simulation included a simulated microphone positioned $0.5 \mathrm{~m}$ normal to the latch center. This was shown previously in Figures 4.17-4.19 by the red markers. The force applied to the striker and bumper were imported from the ED analysis explained earlier. The force from this impact was modelled as action-reaction forces and applied to the approximate area where the components 
would impact in real life. The force was applied to nodal points shown in Figure 4.25 and equally distributed among each node (default setting). The forces analyzed for the $0.851 \mathrm{~m} / \mathrm{s}$ speed and $1.179 \mathrm{~m} / \mathrm{s}$ were $28.77 \mathrm{~N}$ and $42.66 \mathrm{~N}$ respectively. The force was applied in 4 steps over a period of $8 \mathrm{E}-2$ seconds, representative of the amount of time between the start of the primary impact and the end of the fully decayed sound pressure (as measured experimentally). Tables 4.8-4.9 demonstrate the tabular application of the force within ANSYS. The force applied to the bumper was in the negative $\mathrm{x}$ direction and the force applied to the striker was in the positive $\mathrm{x}$ direction.

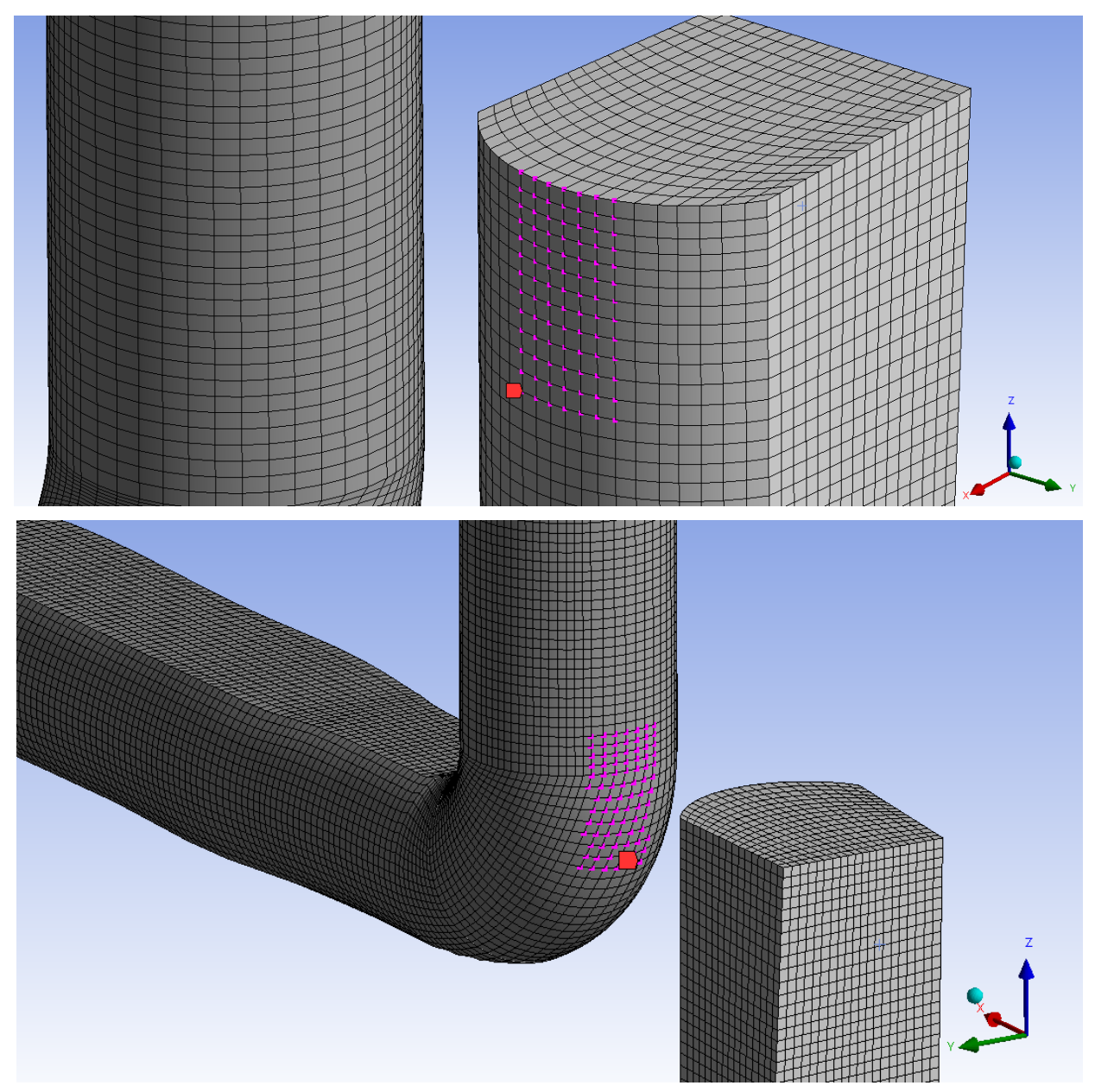

Figure 4.25: Forces applied at nodes for the bumper (Top) and striker

(Bottom). 
Table 4.8: Stepwise application of the force generated during the $0.851 \mathrm{~m} / \mathrm{s}$ entrance speed.

\begin{tabular}{|c|c|c|}
\hline Step & Time $[\mathbf{s}]$ & Force $[\mathbf{N}]$ \\
\hline $\mathbf{1}$ & $1.80 \mathrm{E}-03$ & 0 \\
\hline $\mathbf{2}$ & $3.07 \mathrm{E}-03$ & 28.77 \\
\hline $\mathbf{3}$ & $4.55 \mathrm{E}-03$ & 0 \\
\hline $\mathbf{4}$ & $8.00 \mathrm{E}-02$ & 0 \\
\hline
\end{tabular}

Table 4.9: Stepwise application of the force generated during the $1.179 \mathrm{~m} / \mathrm{s}$ entrance speed.

\begin{tabular}{|c|c|c|}
\hline Step & Time $[\mathbf{s}]$ & Force $[\mathbf{N}]$ \\
\hline $\mathbf{1}$ & $1.80 \mathrm{E}-03$ & 0 \\
\hline $\mathbf{2}$ & $2.98 \mathrm{E}-03$ & 42.66 \\
\hline $\mathbf{3}$ & $4.38 \mathrm{E}-03$ & 0 \\
\hline $\mathbf{4}$ & $8.00 \mathrm{E}-03$ & 0 \\
\hline
\end{tabular}

The fluid material properties of the acoustic region were defined to imitate atmospheric conditions at sea level. Table 4.10 summarizes the properties of the acoustic region for each simulation.

Table 4.10: Material properties of the acoustic fluid domain.

\begin{tabular}{|c|c|}
\hline Acoustic Property & Value \\
\hline Mass Density $\left[\mathbf{k g} / \mathbf{m}^{\mathbf{3}}\right]$ & 1.2041 \\
\hline Speed of Sound $[\boldsymbol{m} / \boldsymbol{s}]$ & 343 \\
\hline Reference Pressure $[\boldsymbol{P a}]$ & $2.00 \mathrm{E}-05$ \\
\hline Reference Static Pressure $[\boldsymbol{k P a}]$ & 101.3 \\
\hline
\end{tabular}

Given the "rectangular-shaped" geometry of the acoustic region for each simulation, an additional boundary condition was applied. Without the proper boundary conditions, the rectangular faces of the geometry would act as solid "walls" allowing internal reflections to occur and be recorded by the microphone. To prevent unwanted reflections, an "acoustic absorption surface" boundary condition was applied to all external faces with the exception of the curved outer surface reserved for the acoustic 
infinite elements. An absorption surface is designed to absorb incident acoustic waves. The level of absorption is dependent on the attenuation coefficient expressed by the relationship shown in Equation 4.10.

$$
\alpha=\frac{I_{\text {absorbed }}}{I_{\text {incident }}}
$$

where $\alpha$ is the attenuation coefficient, $I_{\text {absorbed }}$ is the absorbed sound power and $I_{\text {incident }}$ is the incident sound power. The coefficient ranges were between $0<\alpha<1$ where 1 represents complete sound absorption. To visualize the applied absorption surface, one can imagine acoustic absorption material, similar to the foam material used in the experimental procedure, lining all external boundaries of the acoustic region excluding the curved face. The application of this boundary condition is expressed in Figure 4.26.

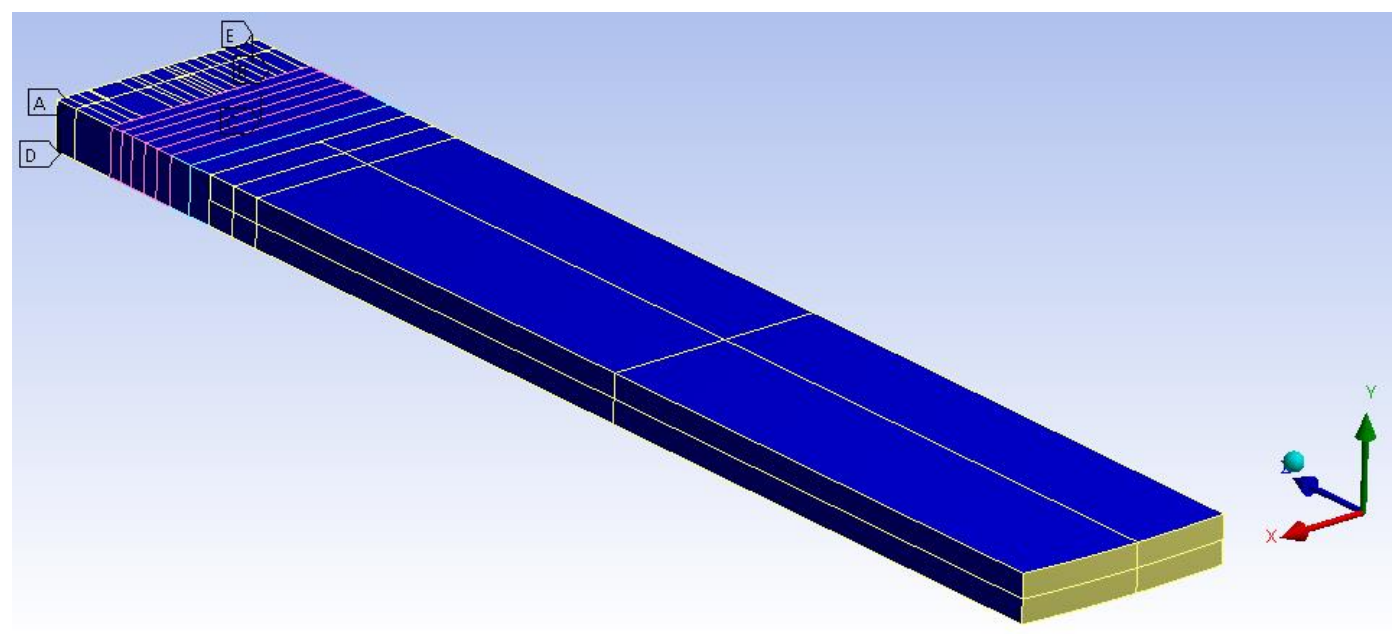

Figure 4.26: Definition of the acoustic absorption surface

The final step in the procedure was to determine the correct sampling frequency for the analysis. ANSYS recommends a sampling that is 20 times the maximum frequency investigated [11]. This recommendation does not fall within the acceptable limits of the workstation. As a result, the sampling frequency was equivalent to the Nyquist frequency represented by Equation 4.11 . 


$$
f_{N}=2 f_{\max }
$$

where $f_{N}$ is the Nyquist frequency, and $f_{\max }$ is the upper frequency investigated limit $(5 \mathrm{kHz})$. The resultant timestep used for each TS acoustic simulation was 1E-4s.

\subsection{Computational Results}

\subsubsection{Simulated Sound Pressure}

A total of six computations were performed. Sound pressure was measured at MP1, MP2 and MP3 as a result of the impact forces from the two impact speeds. Within ANSYS, an "Acoustic Time-Frequency" plot was employed to illustrate the sound pressure measured by the three simulated receivers $0.5 \mathrm{~m}$ away from the latch center. The result is presented in Figure 4.27.

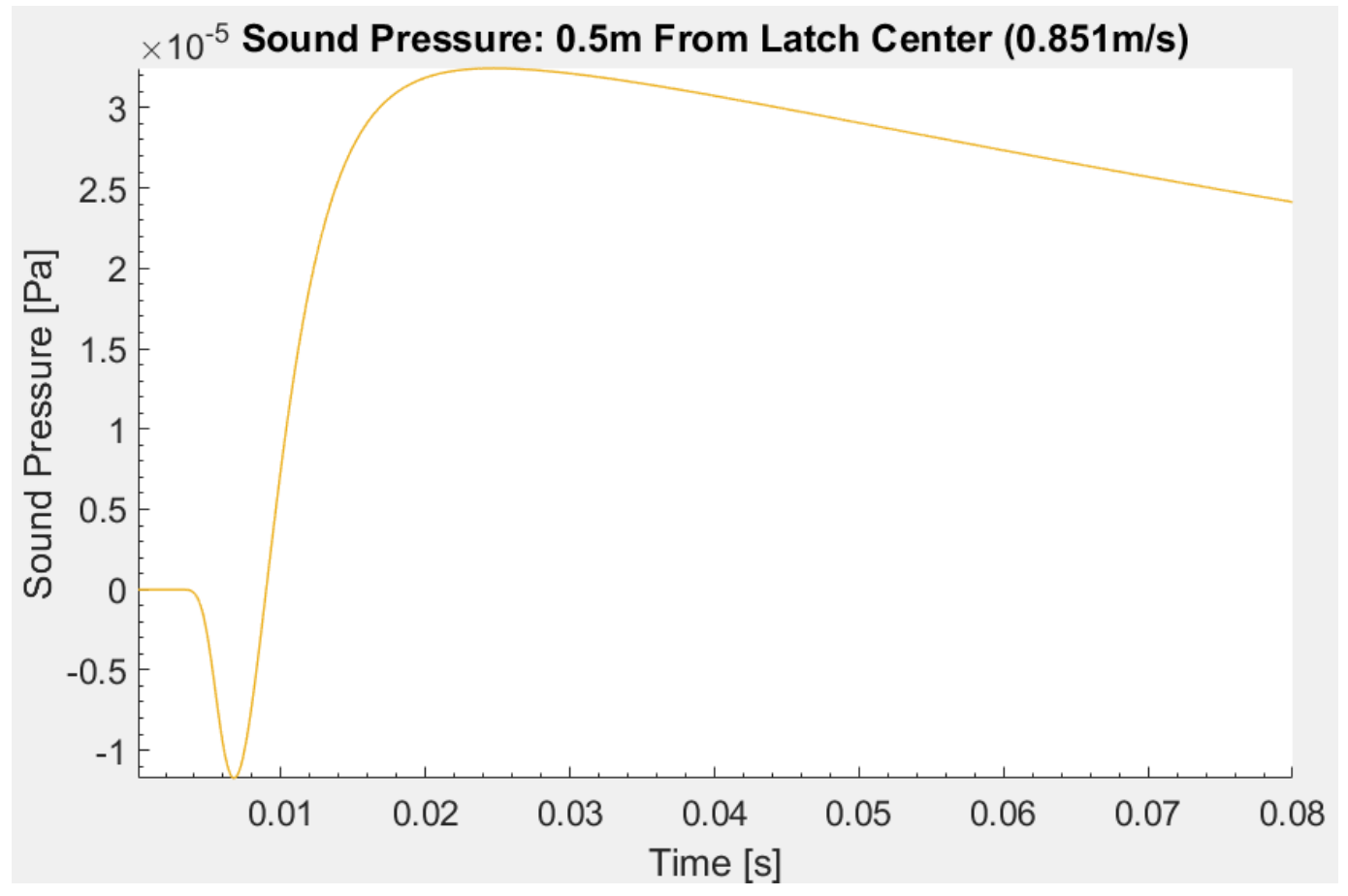

Figure 4.27: Sound pressure measured at MP3 0.5m from the latch. 
This figure reveals that the measured sound pressure in the MP3 position is significantly less compared to the experimental counterpart. In other words, if sound was generated within the acoustic model, it was not able to be measured by the microphone at this location. This trend was also observed at the other microphone locations. With this outcome, two additional microphones were placed within each acoustic model. For the MP3 model, the new receivers were placed at a distance of $0.075 \mathrm{~m}$ and $0.3 \mathrm{~m}$ denoted by MP3_Close and MP3_Mid respectively. This placement was replicated for each acoustic region as shown in Figure 4.28.

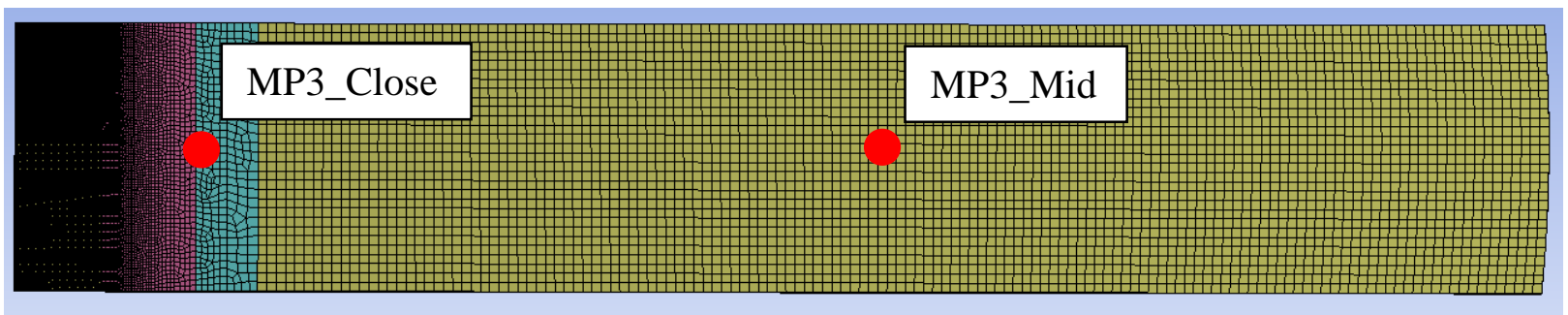

Figure 4.28: Additional microphones added within the acoustic region.

The sound pressure results from MP3_Mid are shown in Figure 4.29. A comparison between this figure and Figure 4.27 shows that more energy is present. Additionally, the sound pressure results from MP3_Close are displayed in Figure 4.30. By comparing the three generated plots, it is evident that the sound pressure recorded at MP3_Close experiences the largest sound pressure. The waveform generated closely resembles the sound pressure waveform present during the experimental analysis. Similarly, the sound pressure results of the closer microphone position from the MP1 and MP2 analysis are presented in Figure 4.31 and Figure 4.32 respectively. The sound pressure results pertaining to the $1.179 \mathrm{~m} / \mathrm{s}$ closing speed are included in the following section. 


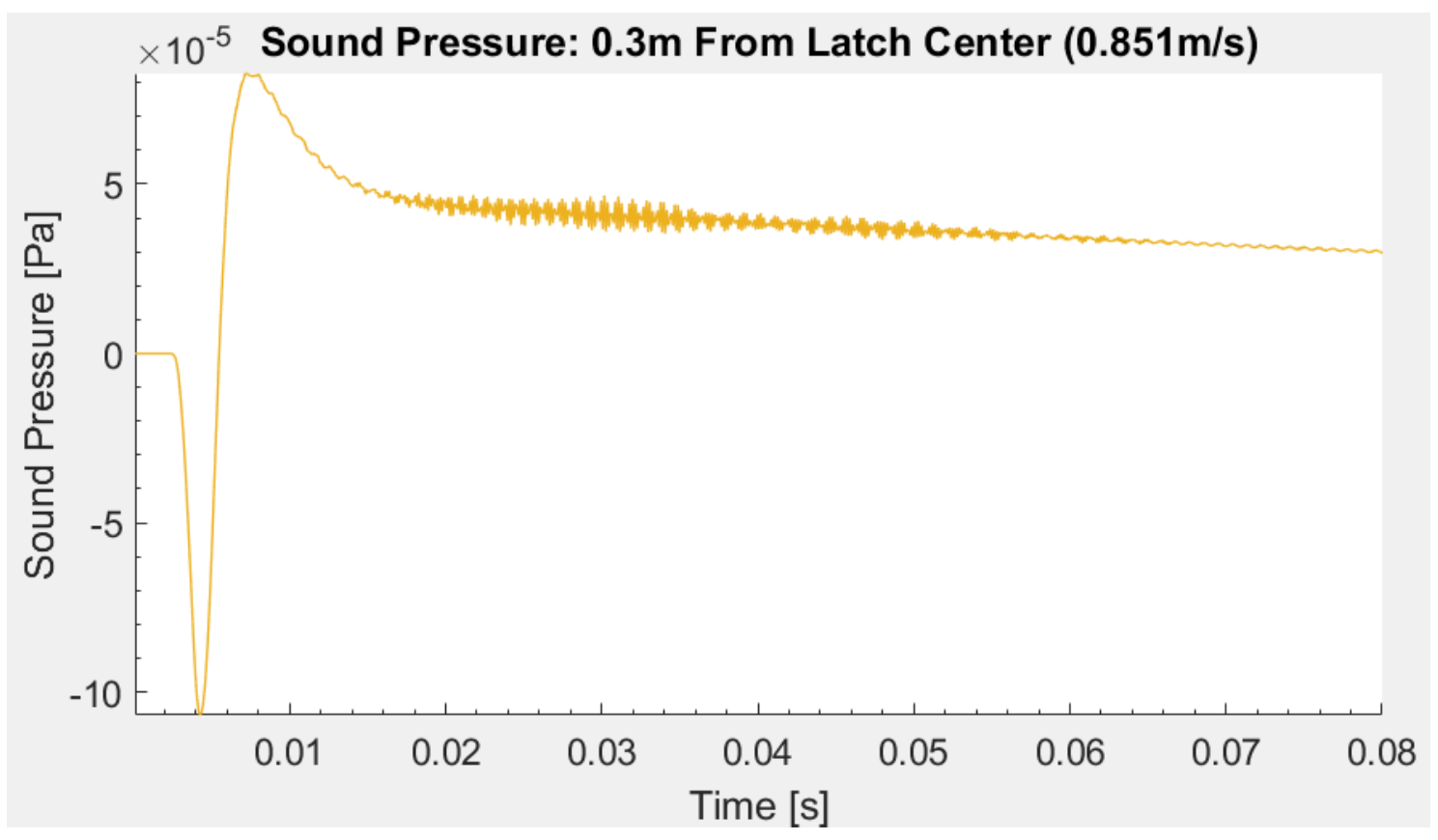

Figure 4.29: Sound pressure result at MP3_Mid.

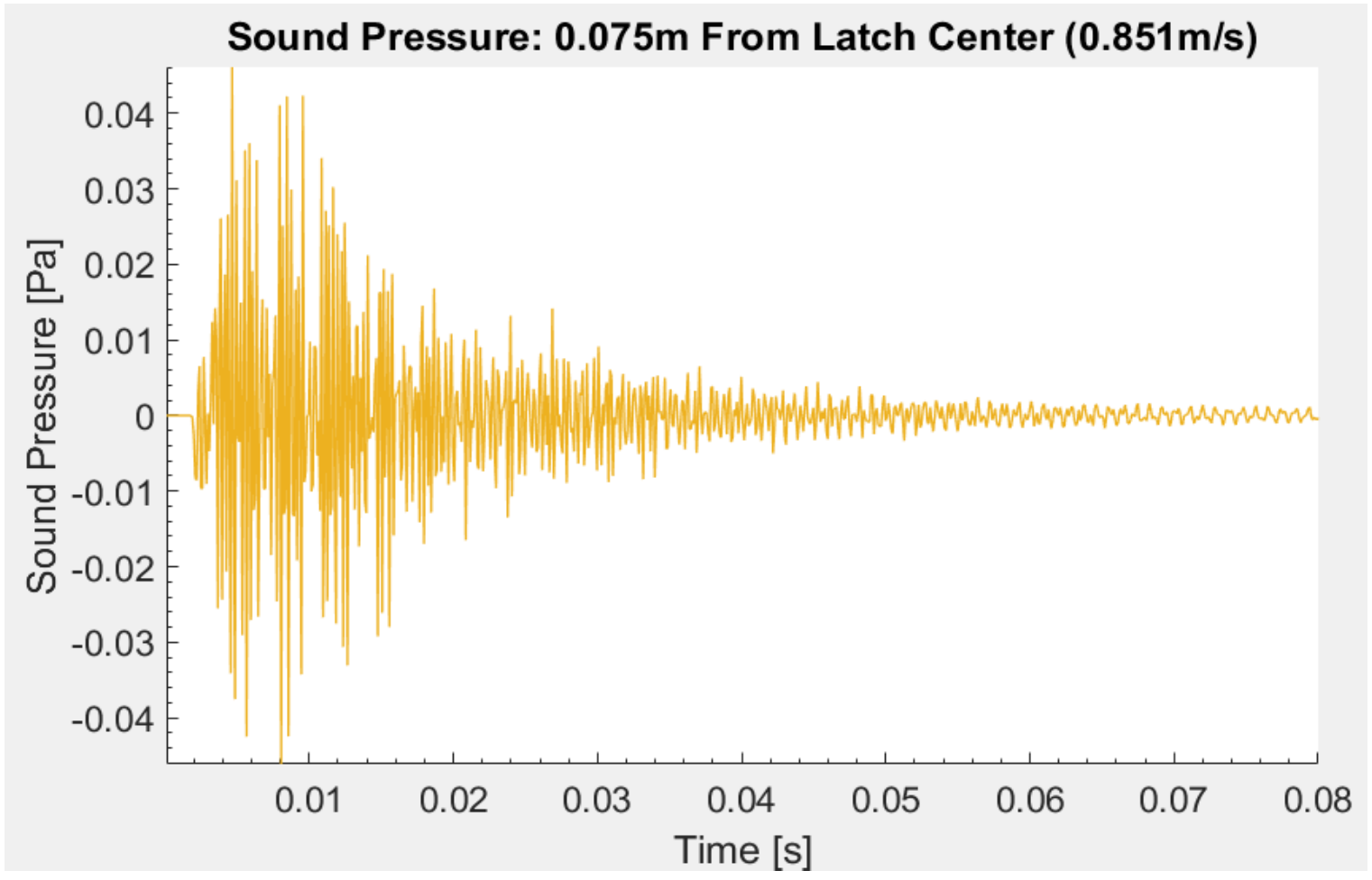

Figure 4.30: Sound pressure results at MP3_Close. 


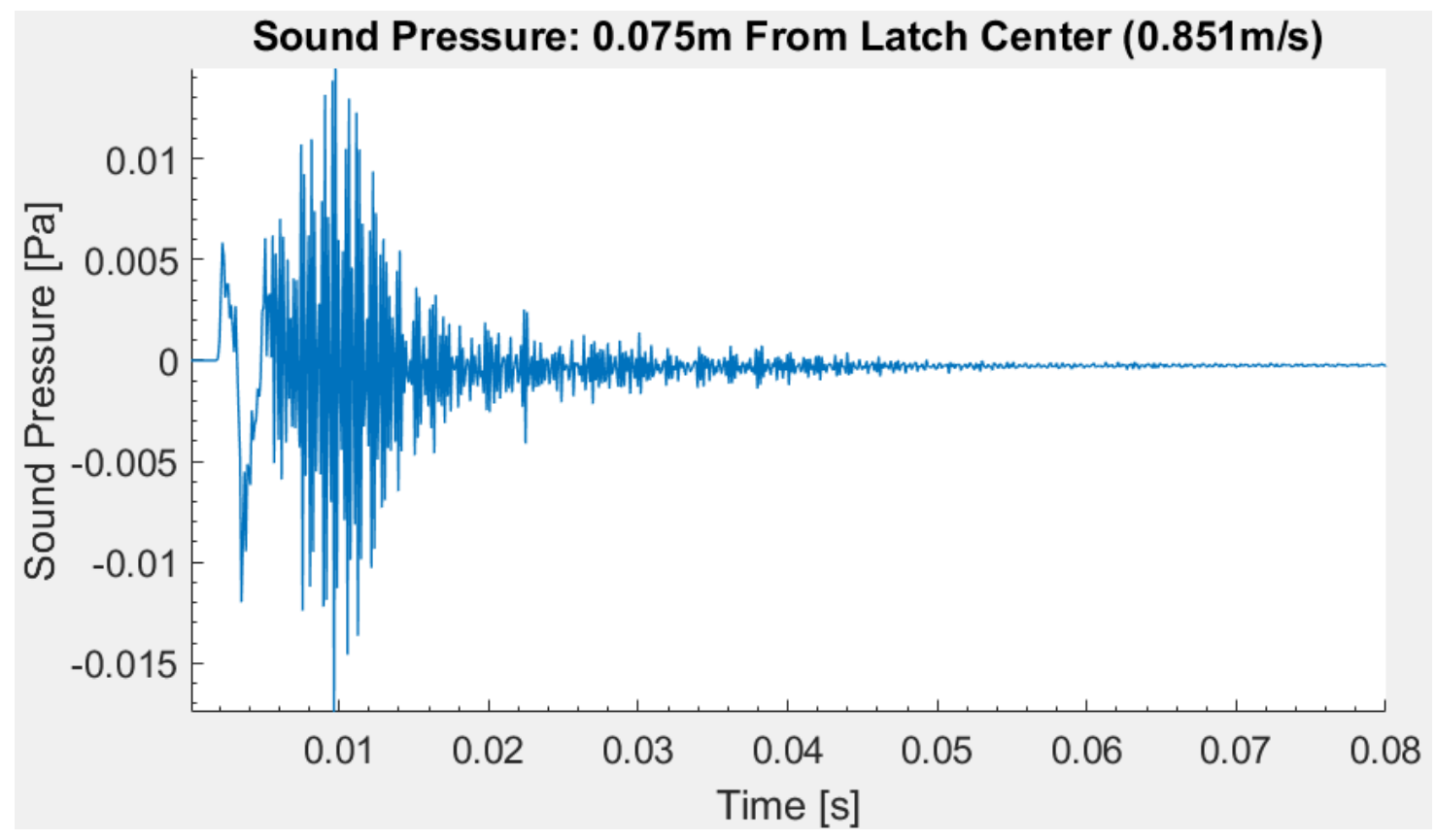

Figure 4.31: Sound pressure result at MP1_Close.

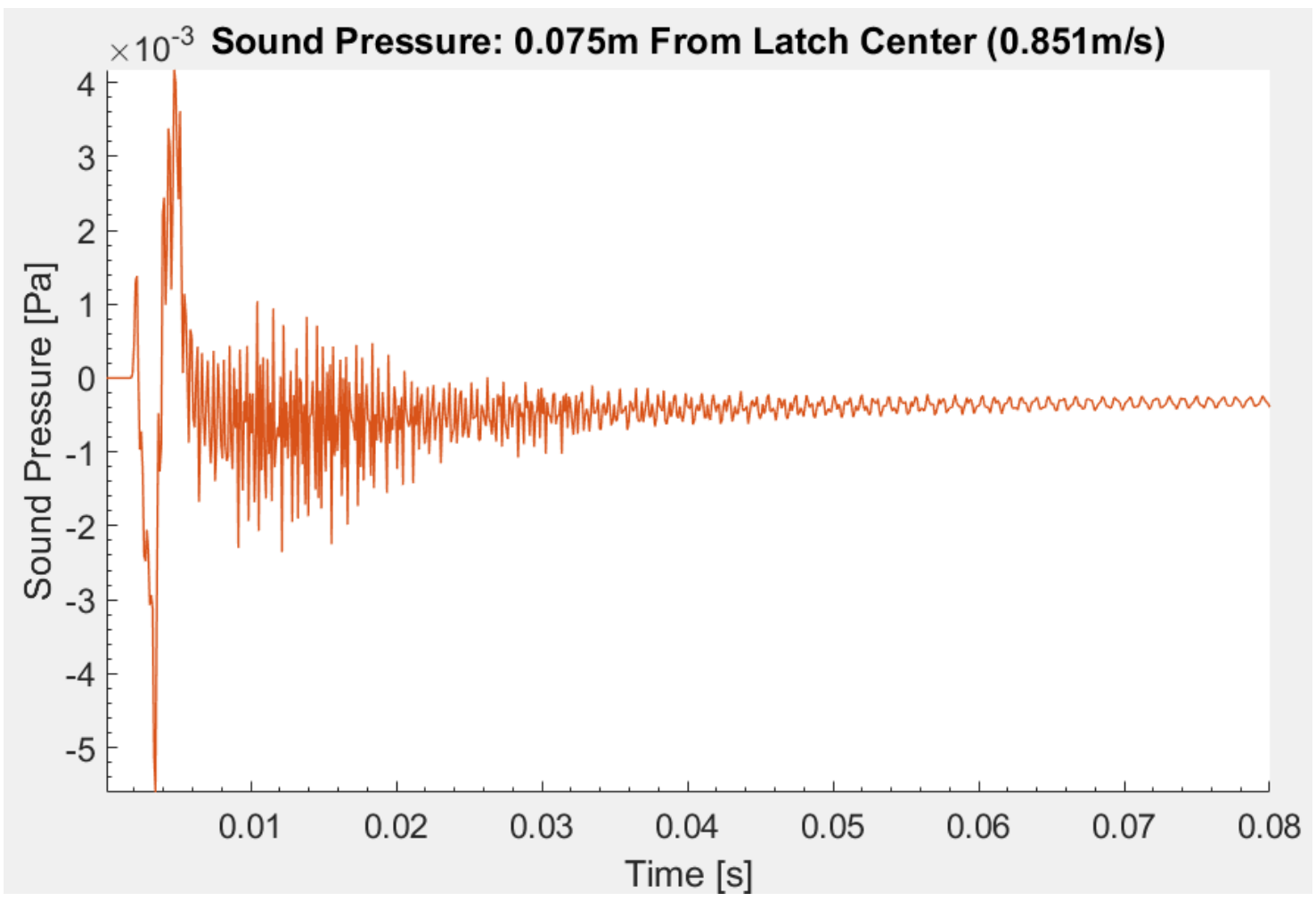

Figure 4.32: Sound pressure result at MP2_Close. 


\subsubsection{Continuous Wavelet Transform of the Simulated Data.}

A spectral composition analysis of the generated sound pressure was performed using the same MATLAB script mentioned in Chapter 3. The results from this analysis are presented in Figures 4.33-4.38.

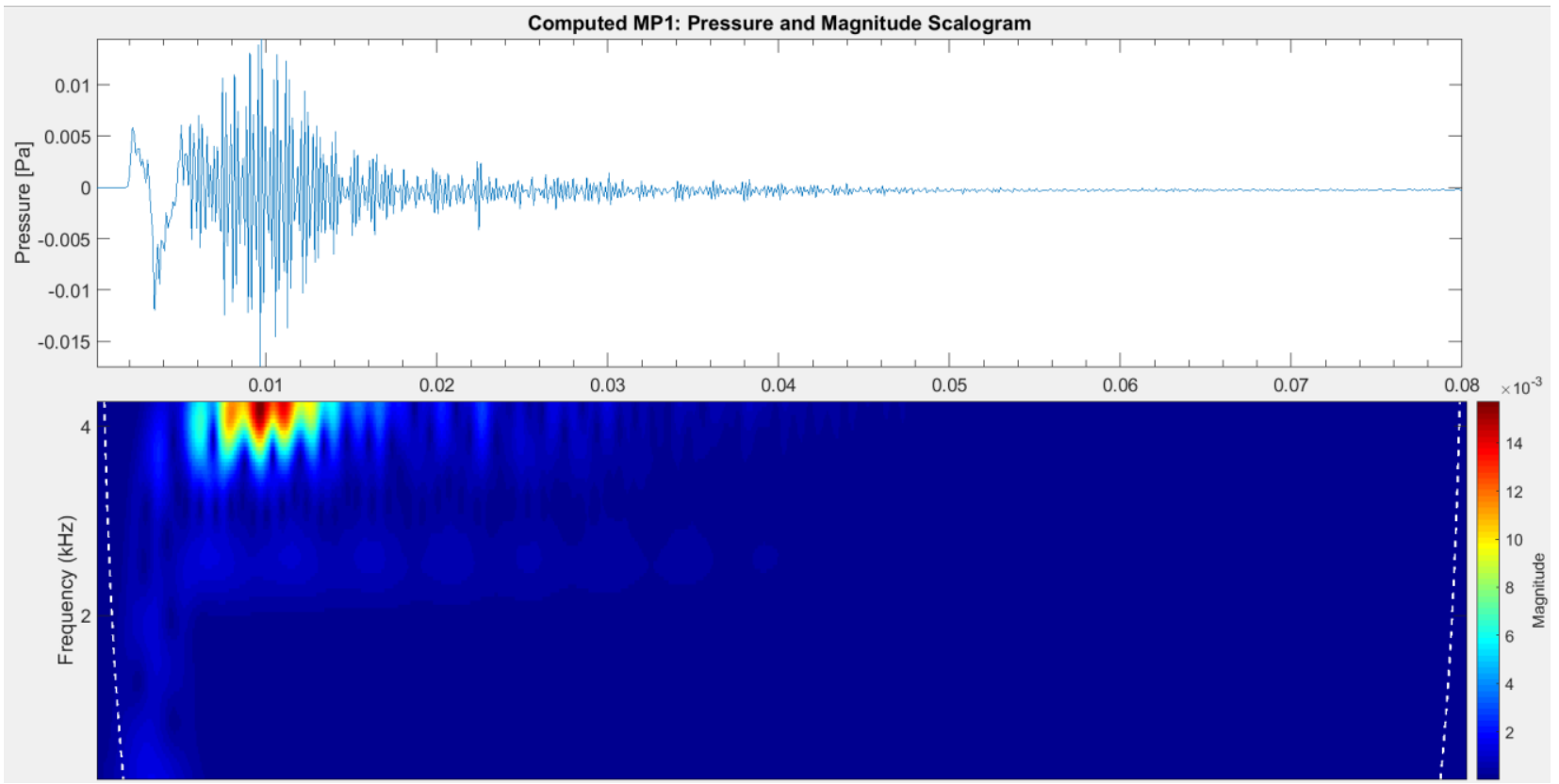

Figure 4.33: Continuous wavelet analysis of the $0.851 \mathrm{~m} / \mathrm{s}$ TS analysis at MP1_Close.

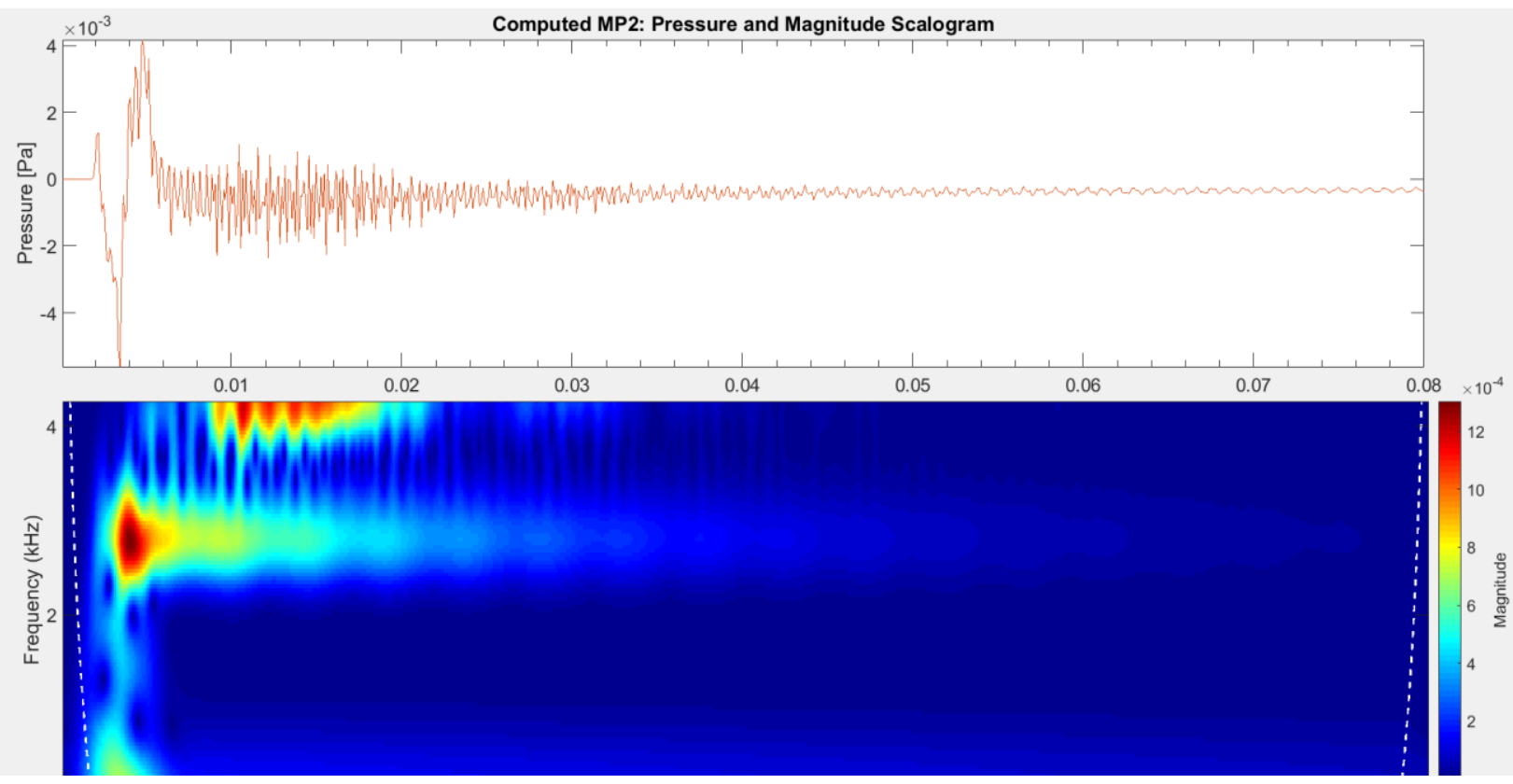

Figure 4.34: Continuous wavelet analysis of the $0.851 \mathrm{~m} / \mathrm{s}$ TS analysis at MP2_Close. 


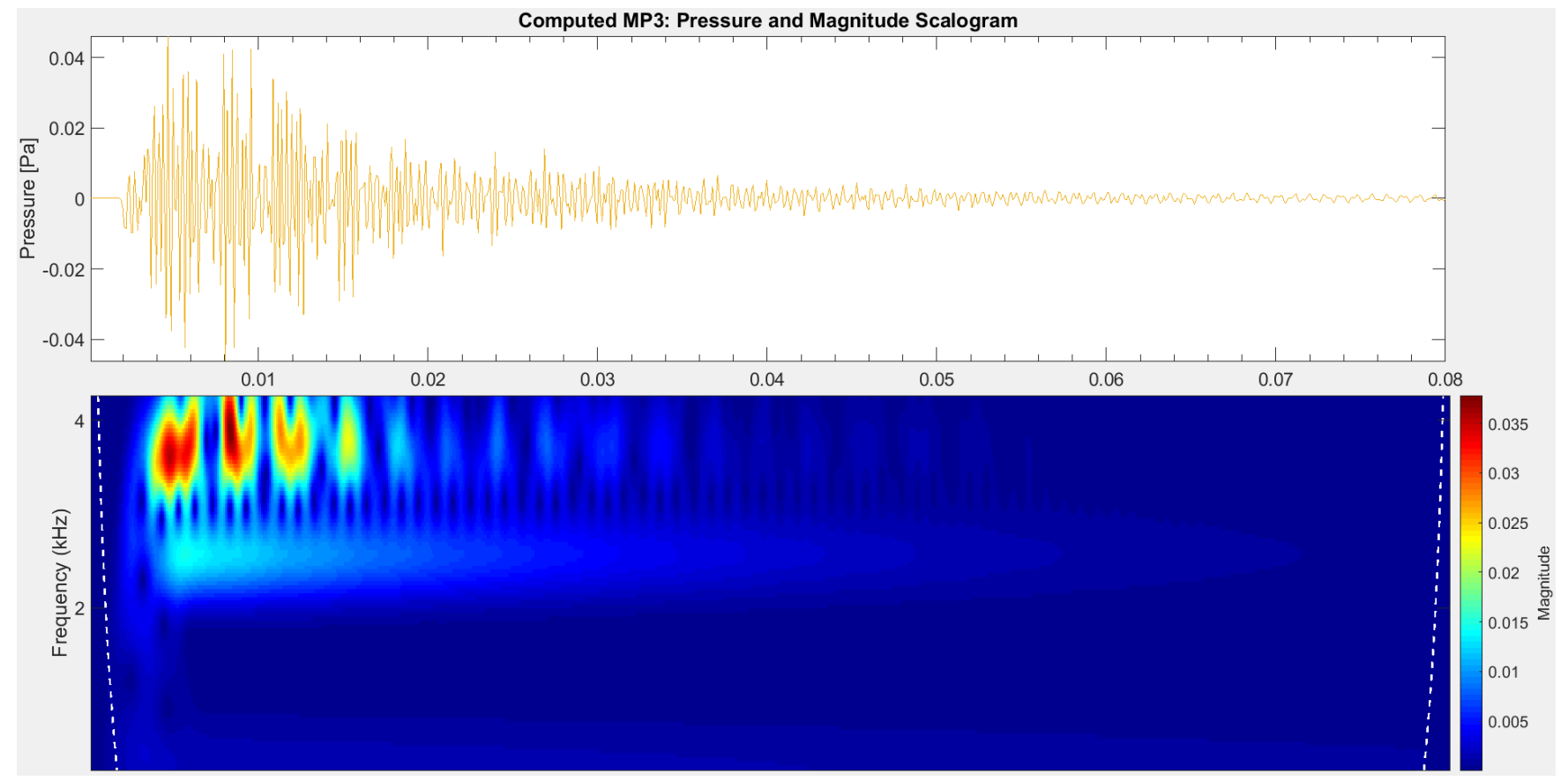

Figure 4.35: Continuous wavelet analysis of the $0.851 \mathrm{~m} / \mathrm{s}$ TS analysis at MP3_Close.

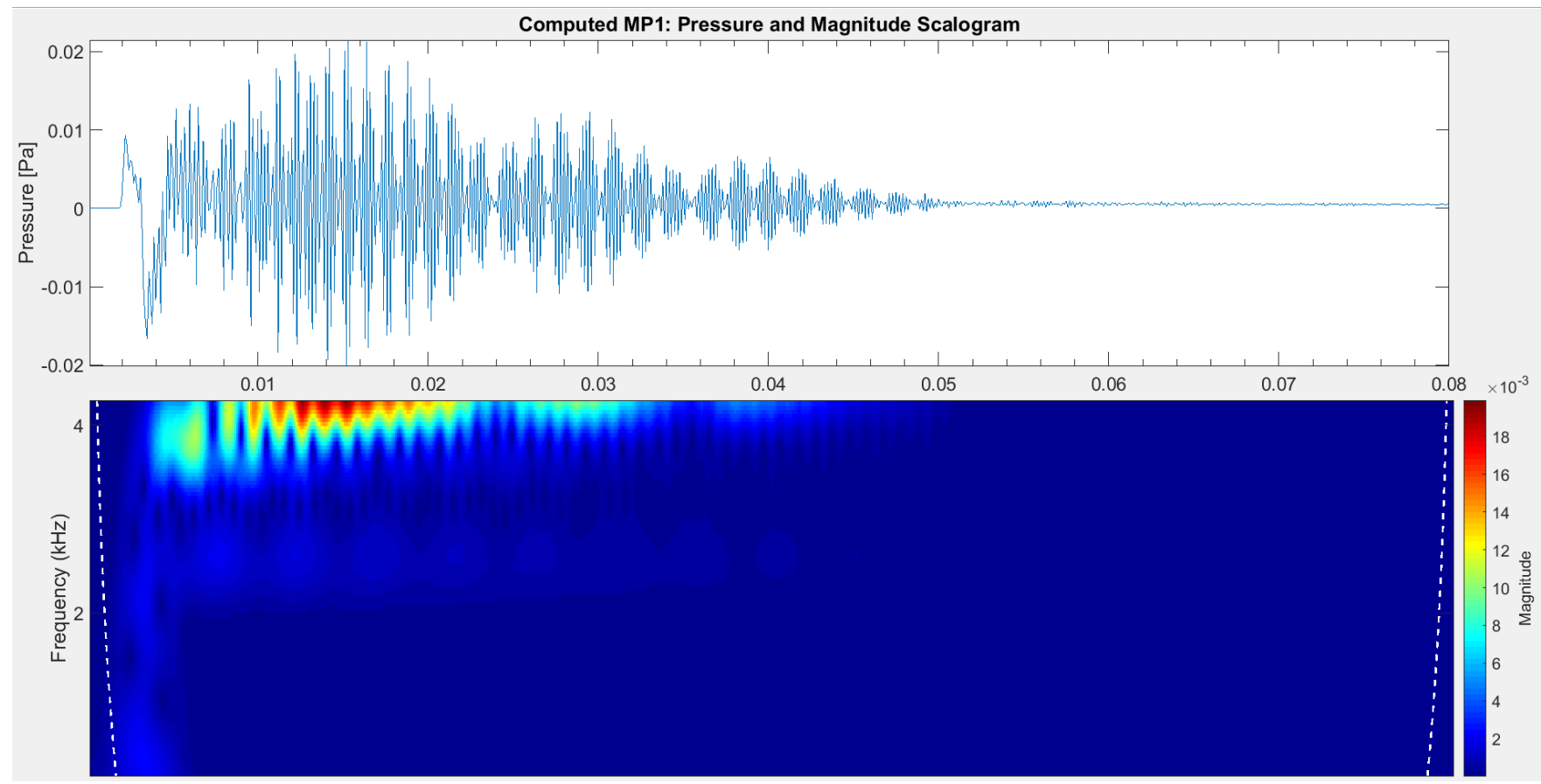

Figure 4.36: Continuous wavelet analysis of the 1.179m/s TS analysis at MP1_Close. 


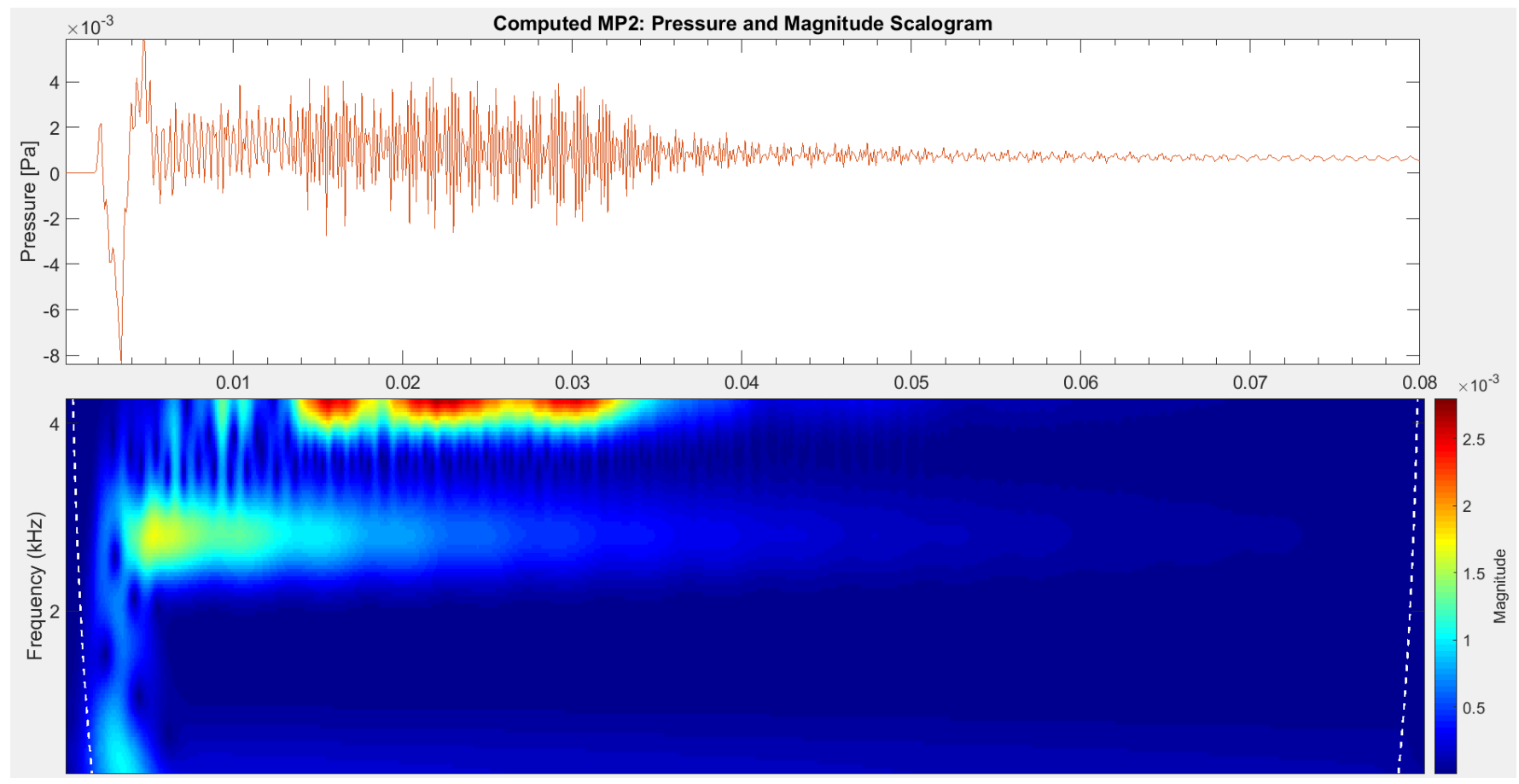

Figure 4.37: Continuous wavelet analysis of the $1.179 \mathrm{~m} / \mathrm{s}$ TS analysis at MP2_Close.

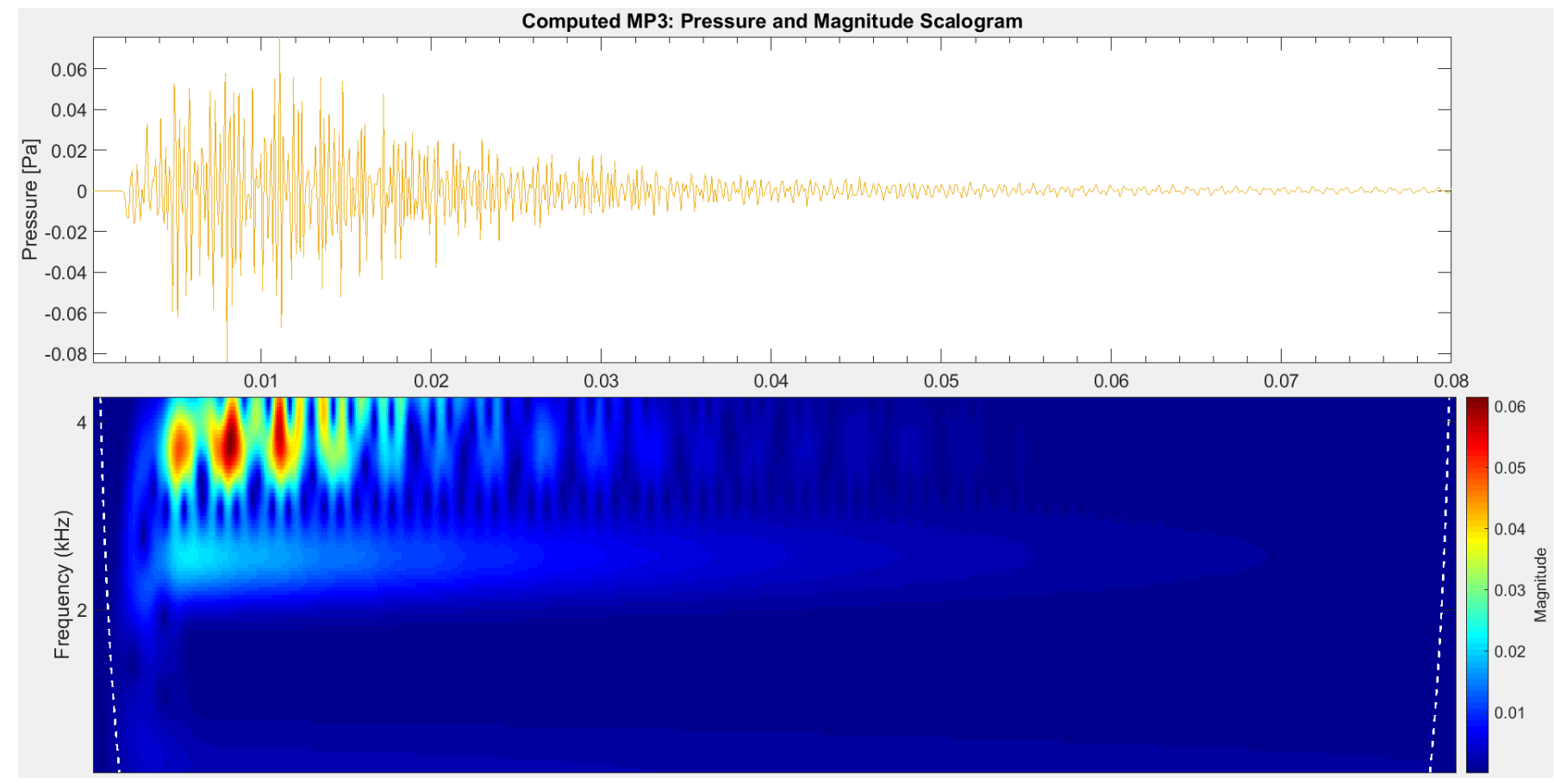

Figure 4.38: Continuous wavelet analysis of the $1.179 \mathrm{~m} / \mathrm{s}$ TS analysis at MP3_Close. 


\subsection{Analysis and Discussion}

This section provides insight into the trends presented in the computational acoustic analysis. Closer observation of the generated data indicates neither of the TS simulations recorded a significant change in sound pressure at $0.5 \mathrm{~m}$ from the latch. However, the inclusion of the additional microphones displays an increase in sound pressure magnitude closer to the latch. The observed trend indicates that as the sound radiates outward towards infinity from the vibrating striker and bumper, the energy within the waveform dissipates before it is able to reach the last receiver.

To obtain a better understanding of the results between the two data sets, a simple breakdown of the physical interaction of the latch components was conducted. By inspection, the sound produced by the latch during the experimental procedure involved the striker physically interacting with the over-slam bumper causing a cascade of impact events and energy transfers within and around the latch. As it was mentioned in the beginning of this chapter, ANSYS was not able to perform a dynamic-acoustic computation due to the method used to model the acoustic fluid. It was also mentioned that FLUID20 elements could displace within the simulation environment subject to an FSI coupling condition. Without this condition, only a pressure degree of freedom is enabled. With that notion, combining a dynamic impact simulation in a fully discretized acoustic fluid results in the degradation of the acoustic elements to a point in which they become degenerate and fail. For this reason, the dynamic and acoustic simulations were performed separately.

It was hypothesized that failure to model the physical impact of the striker with the over-slam bumper does not provide an accurate representation of the real-life dynamics. Previous studies have explored the loud, transient sound generated by impacting objects using a Schlieren Imaging technique [50]. This study observed the presence of weak shock waves emanating from the sound source to the surrounding environment. It was speculated that without the physical impact of the striker and bumper in the computational model, a weak shock could not be generated. 
To reiterate, this explanation is purely speculative as there is no evidence within the recorded experimental sound pressure data suggesting the presence of a weak shock. To prove the existence of a shock, additional testing must be conducted using appropriate instrumentation. This type of analysis was beyond the scope of this investigation, therefore it is recommended to be done in future work.

The simulated waveform data shown in Figure 4.35 and Figure 4.38 are similar in the first 0.014 seconds. This is representative of the sound generated by the primary impact shown in Chapter 3. This difference could be attributed to the exclusion of a "physical" impact in ANSYS. The MP2_Close plot shown in Figure 4.34 and Figure 4.37 displays an atypical sound waveform where there is a single trough at approximately $0.004 \mathrm{~s}$ which then increases to a peak. This behavior is also experienced in MP1_Close (with results that are less extreme) in addition to displaying typical sound waveform characteristics.

Additional observations indicate that the sound propagating toward MP3 possess a larger peak sound pressure compared to MP1_Close and MP2_Close. This could be attributed to the placement of the microphone receivers within the model; MP3 is in line with the latch origin between the striker and bumper. There are no barriers in the sound path between the latch components and the microphone receiver. Conversely, as sound propagates toward MP1_Close and MP2_Close, the latch components act as sound barriers and effect the propagation of sound toward the receivers.

Figure 4.39 displays a comparison of the experimental and computational waveforms at $0.851 \mathrm{~m} / \mathrm{s}$. Initial assessment of these waveforms exhibit a likeness in the overall shape of the waveform, initial deviation from ambient pressure, as well the decay of the sound pressure. However, further inspection shows the magnitude of the experimental data greatly exceeds the magnitude recorded by the computational model. The computational waveform also experiences a slower sound pressure decay rate. 


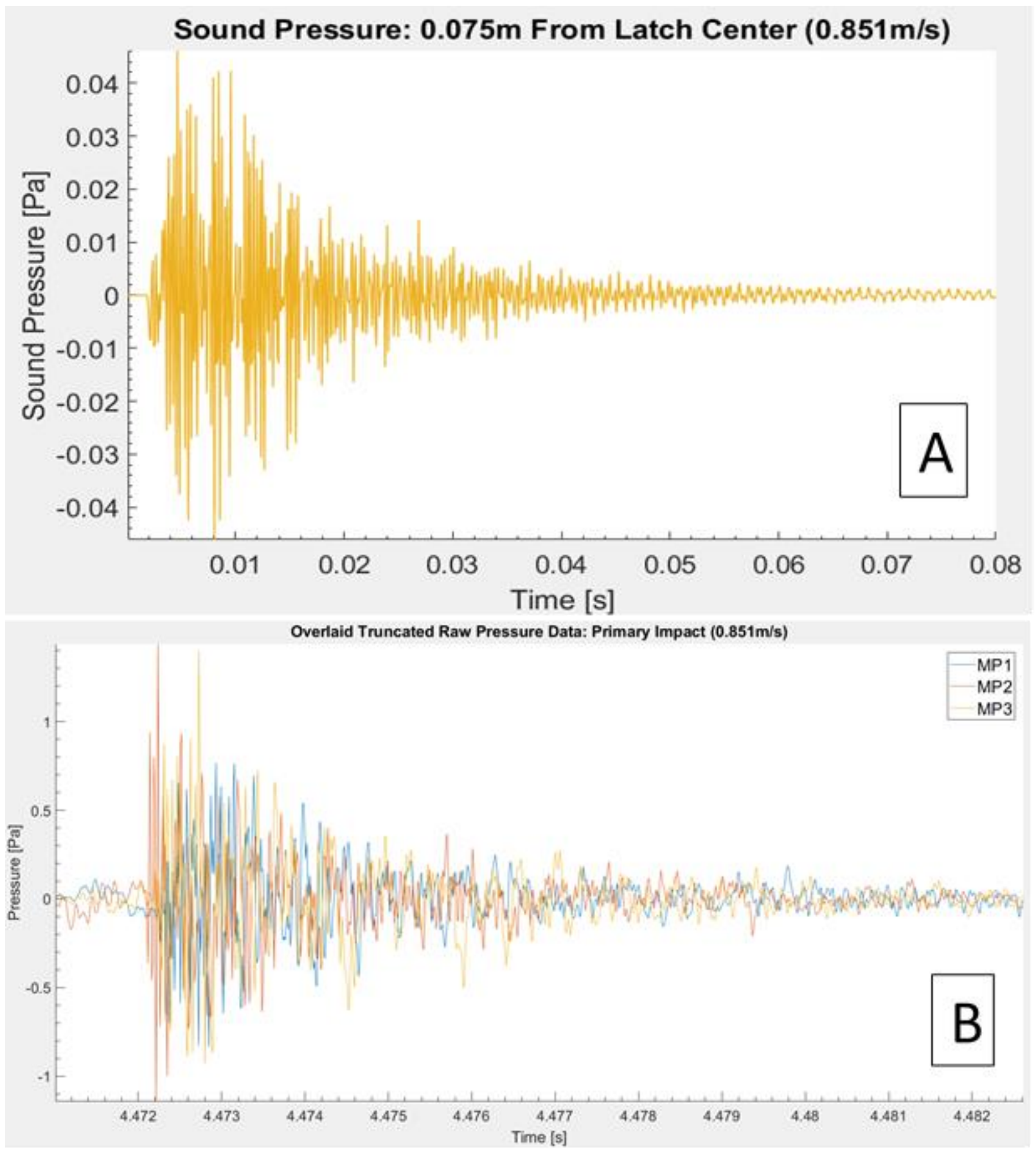

Figure 4.39: Comparison of simulated (A) and experimental (B) primary impact sound pressure of the $0.851 \mathrm{~m} / \mathrm{s}$ closure speed. 
Further analysis of the spectral composition of the simulated sound pressure signal provided insight into the dominant frequency of the waveform as a function of time. Analysis of the CWT results show that the dominant frequency range was between $4 \mathrm{kHz}-5 \mathrm{kHz}$; anything above $5 \mathrm{kHz}$ was not investigated.

Interestingly, there was additional contribution from the $2.3 \mathrm{kHz}-2.7 \mathrm{kHz}$ bandwidth with several simulated waveforms. Recalling the experimental CWT plots presented earlier (Figure 3.23-3.25), the results indicate a dominate frequency range of $3.8 \mathrm{kHz}$ to $5 \mathrm{kHz}$ during the primary impact event. With this comparison, it can be concluded that close similarities between the experimental and computational data are present. It is also apparent that the dominant frequencies in both MP3_Close plots possess higher normalized magnitudes as compared to the other microphones at the same speed. This result is not unexpected since it was mentioned previously that the sound pressure measured at MP3_Close was larger compared to the other microphones.

It is apparent that error was present within the computational analysis. The error associated with the RBD analysis was carried over to the ED analysis which was then carried over to the TS acoustic analysis. This caused the error to accumulate with each module, further reducing the accuracy of the results at each step. It is believed a majority of the error occurred within the TS structural acoustic analysis as it contained the most assumptions and simplifications compared to the other simulation modules. It was also the only simulation that frequently failed as the matrix formulation and solution procedure required more hard-disc space than what was locally available.

To increase the accuracy of each simulation module, each analysis setting would have to be set to a specific value depending on the nature of the simulation, the geometry of the model, and input parameters. However, due to the inherent limitations of the workstation as well as the focus of this study, the assumptions and simplifications made to provide approximated results were deemed acceptable. It is recommended in future work that each computational analysis be carefully formulated in order to reduce the magnitude of accumulated error. 
Mass scaling is used in ED analysis to scale up the density of certain elements (typically the smallest elements) to reduce the solving time required for the model. This is common in industry, especially for models containing small, complex geometry that can dictate the overall solution time [45]. Theoretically, the application of mass scaling to a model should not affect the accuracy of the results as it should only improve the solution run time. However, by increasing the mass of the model, it may affect the inertial properties of the interaction components, which could alter the reaction forces of the impact. For this study, mass scaling was not investigated. However, it is encouraged that future work investigate the effect of mass scaling with regard to computational run time and result accuracy.

Given all the simplifications presented throughout the chapter and the difficulty modelling the "physical impact" between the striker and bumper, it is advised that the simulated acoustic results be interpreted with caution. Given the many steps involved to conduct an acoustic analysis, this study should prove to be an excellent resource for future work. However, it is suggested that another acoustic simulation package be used to perform transient acoustic simulations. 


\section{Chapter 5}

\section{Conclusions and Future Work}

Sound pressure data from an automotive door closure system was collected in a semi-anechoic chamber to inspect the frequency content of the radiated acoustic response. Two closing speeds of $0.851 \mathrm{~m} / \mathrm{s}$ and $1.179 \mathrm{~m} / \mathrm{s}$ were used to engage the striker and the latch assembly. A vertical double pendulum assembly was used to simulate the door closing motion required to successfully engage the latch. By inspection of the sound waveforms, two distinct impact events were observed. These events were denoted as the primary and secondary impact with the assumption that the aural response of the latch was influenced by these two impact events.

Post processing methodologies such as a Continuous Wavelet Transform analysis and Sound Pressure Level versus 1/3 Octave Bandwidth were employed. These methodologies were used to determine the spectral composition as a function of time and of the overall recorded sound sample respectively. The additional kinetic energy from the striker caused lower frequencies to become more pronounced in the CWT compared to the CWT of the slower speed. Introducing more energy into the system resulted in an average $11.35 \mathrm{dBA}$ increase in the SPL within the $160 \mathrm{~Hz}$ to $5 \mathrm{kHz}$ bandwidth.

From the ANSYS Rigid Body Dynamic and Explicit Dynamic models, the impact event between the ratchet and housing as well as the striker and over-slam bumper generated the greatest reaction forces within the latch. It was concluded that these impact events greatly influenced the overall acoustic response. The Striker/Over-Slam Bumper event was identified as the primary impact and the Ratchet/Housing event was identified as the secondary impact. An ANSYS Transient Structural analysis was performed in an attempt to correlate the experimental sound pressure results with simulated results. Due to inherent limitations of the computational workstation, only the primary impact was modelled as it was observed to have the greatest influence on the overall sound. 
Steps were taken to accurately model the dynamics of the impact in a simulated free field environment. Sound pressure results were collected and analyzed using a CWT analysis. The results displayed similarities with the dominant frequencies experienced during the experimental test. However, the results of the computational analysis should be observed with caution as it was not possible to model the "physical impact" of the striker/bumper interaction. This limitation, in addition to the accumulated error during each computational analysis, caused inconsistencies between the experimental and computational methods.

Given all the limitations and simplifications of the test apparatus, experimental procedure and computational model, it can be concluded that the overall acoustic phenomena projecting from the latch based on impact events of the internal components is influenced by the closing speed of the striker.

If automotive designers incorporated the findings from this study into future vehicle designs, there would be advantages and disadvantages present. The advantage would be the generation of a more pleasing sound which would positively influence a customer's perception of the product's quality and potentially lead to increased product sales. The disadvantage would be the additional energy absorbed by the latch during the closing procedure. This excess energy would expedite the wearing process of the latch internal components, resulting in a shorter lifespan of the latch. Furthermore, this extra energy could potentially shorten the lifespan of other subsystems in the vehicle door assembly such as power windows, audio systems, and proximity sensors.

Although this experiment provided insight into the effects of door closing speeds and the latch acoustic phenomena, there is not enough evidence to definitively confirm that a faster closing speed will produce a more preferred aural response. These initial results warrant further investigation to determine the relationship between closing speed and the frequency composition of the radiated sound. 
It should be mentioned that this was a highly idealized study. Only a subset of the latch components was analyzed to better understand the complex component interactions and their effect on the overall sound. The computational analysis did not account for the other component interactions that were indirectly affected by the striker. It is suggested to investigate the other impact events present during latch operation to obtain a better understanding of the acoustic phenomena. Additionally, this experiment was conducted on the latch assembly exclusively. To obtain a more accurate representation of the sound that a consumer would hear, it is recommended that any future experimental tests be performed on a latch affixed to the frame assembly of a vehicle.

As evidenced by the results presented in this thesis, the computational analysis was a complex and arduous process. Separate analyses generated results that were used as initial conditions for another analysis. The error associated with the result was also carried over to the second analysis and again to the third analysis, attributing to an increase in overall error.

These errors resulted from the inherent limitations of the workstation, the complex geometry of the automotive door closure, and the methods used for the computational analysis. With that notion, it is suggested that future work include a systematic approach to quantify the errors at each stage of the computational modelling and compare them to the experimental results. This comparison would ensure the computational results at each stage are a correct representation of the real-life dynamics and radiating acoustic response of the door closure.

The conclusion of this study elicits many thought-provoking questions that provide motivation for future work: How will the acoustic response change if the closing speed was larger or smaller than the closing speeds used? Are certain frequencies more susceptible to a change in closing speed than others? Would a similar result be found if different materials were used? It is recommended that future work further investigates the proposed questions as well as include a computational acoustic analysis that is capable of modeling "physical impacts" within the computational environment to better represent the complex 
interaction events present during latch operation. After these suggested topics for future research are addressed, additional work could investigate other acoustic metrics such as sound intensity and sound power of the door closure. Based on the findings of this study, in addition to the findings from future studies, modifications to the door closure structure could be investigated in an effort to achieve a more desired acoustic response. 


\section{References}

[1] M. Brinkmeier, U. Nackenhorst, J. Biermann, and O. Von Estorff, "Simulation and Analysis of Tire Road Noise, Finite Element Results and Validation,” J. Acoust. Soc. Am., vol. 123, no. 5, pp. 3673-3673, 2008.

[2] T. Yasuda, C. Wu, N. Nakagawa, and K. Nagamura, "Predictions and Experimental Studies of the Tail Pipe Noise of an Automotive Muffler Using a One Dimensional CFD Model,” Appl. Acoust., vol. 71, no. 8, pp. 701-707, 2010.

[3] J. Trevelyan and J. Yang, "Vehicle Door Panel Reinforcement Design to Reduce Wind Noise," in ABAQUS User's Conference, 1999, p. 3.

[4] J. G. Ih, H. J. Kim, S. H. Lee, and K. Shinoda, "Prediction of Intake Noise of An Automotive Engine in Run-Up Condition,” Appl. Acoust., vol. 70, no. 2, pp. 347-355, 2009.

[5] H. Fastl and E. Zwicker, Psychoacoustics: Facts and Models, Third Edit. Springer International Publishing, 2007.

[6] T. D. Rossing, Springer Handbook of Acoustics, First Edit. Springer International Publishing, 2014.

[7] Bruel \& Kjaer, "Measuring Sound," ASHRAE J., vol. 47, no. 1, pp. 4-8, 2005.

[8] B. Goelzer, H. Colin, and A. Gustav, "Occupational Exposure to Noise: Evaluation, Prevention and Control," Fed. Inst. Occup. Saf. Heal., p. 334, 2001.

[9] M. Petersen, "Musical Analysis and Synthesis in Matlab," Coll. Math. J., vol. 35, no. 5, pp. 396401, 2004.

[10] G. M. Ballou, Handbook for Sound Engineers, Fourth Edi. Focal Press, 2008.

[11] C. Q. Howard and B. S. Cazzolato, Acoustic Analysis Using MATLAB and ANSYS. CRC Press, 2015.

[12] K. Namba, S. Sonoko, "Relation Between Envelope Pattern and Sound Quality of Impulsive Sounds," J. Acoust. Soc. Am., vol. 20, no. 2, pp. 51-53, 1999. 
[13] K. Genuit, "Vehicle Interior Noise - Combination of Sound, Vibration and Interactivity," Sound and Vibration, p. 5, 2009.

[14] K. Becker, S. J. Walsh, and J. Niermann, "Time Frequency Analysis of Transient Nvh Phenomena in Vehicles," in Proceedings of the XVth German-Polish Scientific Seminar, 2003, pp. 100-104.

[15] J. M. Lilly and S. C. Olhede, "Generalized Morse Wavelets as a Superfamily of Analytic Wavelets," IEEE Trans. Signal Process., vol. 60, no. 11, pp. 6036-6041, 2012.

[16] S. Kuwano, H. Fastl, S. Namba, S. Nakamura, and H. Uchida, "Quality of Door Sounds of Passenger Cars," Acoust. Sci. Technol., vol. 27, no. 5, pp. 309-312, 2006.

[17] Transport Canada, Door Locks and Door Retention Components, vol. 75, no. 33. Canada, 2010, p. 7370.

[18] D. M. Howard and J. A. S. Angus, Acoustics and Psychoacoustics, 4th ed. Focal Press, 2009.

[19] G. Karki, "Human Ear: Structure and Anatomy," 2018. [Online]. Available: http://www.onlinebiologynotes.com/human-ear-structure-anatomy/.

[20] J. Treasure, Sound Business, Second Edi. Management Books 2000 Ltd., 2007.

[21] V. R. Madgazin, “The Information Theory of Emotions of Musical Chords," Cornell University Library. p. 18, 2009.

[22] M. Höchstetter, J. Sautter, and U. Gabbert, "Predicting the Perceived Quality of Impulsive Vehicle Sounds," in EuroNoise 2015, 2015, pp. 2411-2416.

[23] ASA, ASA S1.1: American National Standard Acoustical Terminology. 2013, p. 1.

[24] HEAD Acoustics, "Loudness and Sharpness Calculation with ArtemiS," in HEAD acoustics GmbH, 2006, vol. 1, pp. 1-13.

[25] S. Kuwano, H. Fastl, S. Namba, S. Nakamura, and H. Uchida, "Subjective Evaluation of Car Door Sound," in Proceedings of the Sound Quality Symposium, 2002, pp. 6-10.

[26] J. Hyeonho, W. Seong, H. Lee, S. Kim, D. Park, and Y. June, “A Study On Sound Quality Evaluation Index of Car Door Latch and Improving Sound Quality by Modifying Door Latch 
Assembly Design," in International Congress and Exposition on Noise Control Engineering, 2014, vol. 25, no. 02 , pp. $1-8$.

[27] H.-W. Beckmann, R. Draier, O. Von Estorff, K. Klamt, M. Markiewicz, and O. Zaleski, “Modellierung eines Waschautomaten für vibroakustische Untersuchungen," in CADFEM User's Meeting, 2004, no. January 2004.

[28] O. Von Estorff, "Challenges in Technical Acoustics: What Can Be Computed Today," in DYNAmore GmbH, 2008, pp. 23-36.

[29] O. Von Estorff, U. Nackenhorst, and S. Petersen, "Numerical Analysis of Tire Rolling Noise Radiation: Computational Aspects," in EuroNoise, 2003, vol. 89, no. SUPP., pp. 0-6.

[30] D. Bies and C. Hansen, Engineering Noise Control: Theory and Practice, vol. 3. Spon Press, 2009.

[31] G. T. V Garro and C. K. Mechefske, "Experimental and Computational Methods for Investigating Automotive Door Closure Sounds," in Proceedings of the ASME Design Engineering Technical Conference, 2018, pp. 1-10.

[32] McMaster-Carr, "Contoured Sound-Absorbing Sheet with Adhesive Backing," 2018. [Online]. Available: https://www.mcmaster.com/\#9710t31/=1d84gx6. [Accessed: 08-Mar-2018].

[33] C. Couvreur, “Octave - MATLAB File Exchange,” MATLAB, 1997. [Online]. Available: https://www.mathworks.com/matlabcentral/fileexchange/69-octave. [Accessed: 10-Mar-2018].

[34] S. A. Gelfand, Hearing: An Introduction to Psychological and Physiological Acoustics, Fifth Edit. Informa Healthcare, 2010.

[35] International Organization for Standardization, ISO 226:2003(E): Acoustics - Normal EqualLoudness-Level Contours. 2003, p. 8.

[36] International Electrotechnical Commission, IEC 61672-1: Electroacoustics- Sound Level Meters Part 1: Specifications. 2002, p. 14.

[37] International Electrotechnical Commission, IEC 61260-1: Electroacoustics - Octave-Band and 
Fractional-Octave-Band Filters - Part 1: Specifications. 2014, p. 5.

[38] M. Misiti, Y. Misiti, G. Oppenheim, and J.-M. Poggi, MATLAB Wavelet Toolbox Getting Started Guide. 2018.

[39] K. Niermann, J. Walsh, S.J., Becker, "Wavelet Analysis of Vehicle Door Closure Sounds," in Proceedings of the Institute of Acoustics, 2003, vol. 25, no. 3, pp. 157-164.

[40] M. Misiti, Y. Misiti, G. Oppenheim, and J.-M. Poggi, MATLAB Wavelet Toolbox User's Guide. 2018.

[41] I. Calimanescu, N. Buzbuchi, and A. Dima, "Explicit Dynamic Simulation of a Space Debris Projectile Impact Over a Satellite Structure,” J. Mar. Technol. Environ., vol. 1, pp. 71-80, 2009.

[42] D. Dreyer, S. Petersen, and O. von Estorff, "Effectiveness and Robustness of Improved Infinite Elements For Exterior Acoustics," Comput. Methods Appl. Mech. Eng., vol. 195, no. 29-32, pp. 3591-3607, 2006.

[43] ANSYS, “Ansys Application Customization Toolkit Store,” 2015. [Online]. Available: https://appstore.ansys.com/download?prodid=APC-ACTAPP-177.

[44] ANSYS, ANSYS Mechanical APDL Element Reference. 2013.

[45] ANSYS, “ANSYS Explicit Dynamics Analysis Guide,” no. August. ANSYS, 2016.

[46] ANSYS, “Mesh Quality \& Advanced Topics Introduction to ANSYS Meshing,” 2014.

[47] ANSYS, Introduction to ANSYS Acoustics ACT R17. 2016.

[48] ANSYS, ANSYS Mechanical Linear and Nonlinear Dynamics. 2017.

[49] Ansys, "Lecture 3: Introduction to Contact," ANSYS: Mechanical Structural Nonlinearities. ANSYS, p. 74, 2010.

[50] M. J. Hargather, G. S. Settles, and M. J. Madalis, "Schlieren Imaging of Loud Sounds and Weak Shock Waves in Air Near the Limit of Visibility," Shock Waves, vol. 20, no. 1, pp. 9-17, 2010. 


\section{Appendix A}

\section{Decibel Scale}

The audible sound pressure range is very large between 20micro pascal and 100pascal. Studies have proven that human ear responds logarithmically to sound changes. To model this, the decibel scale was introduced which exhibits a sound pressure level for a given sound pressure.

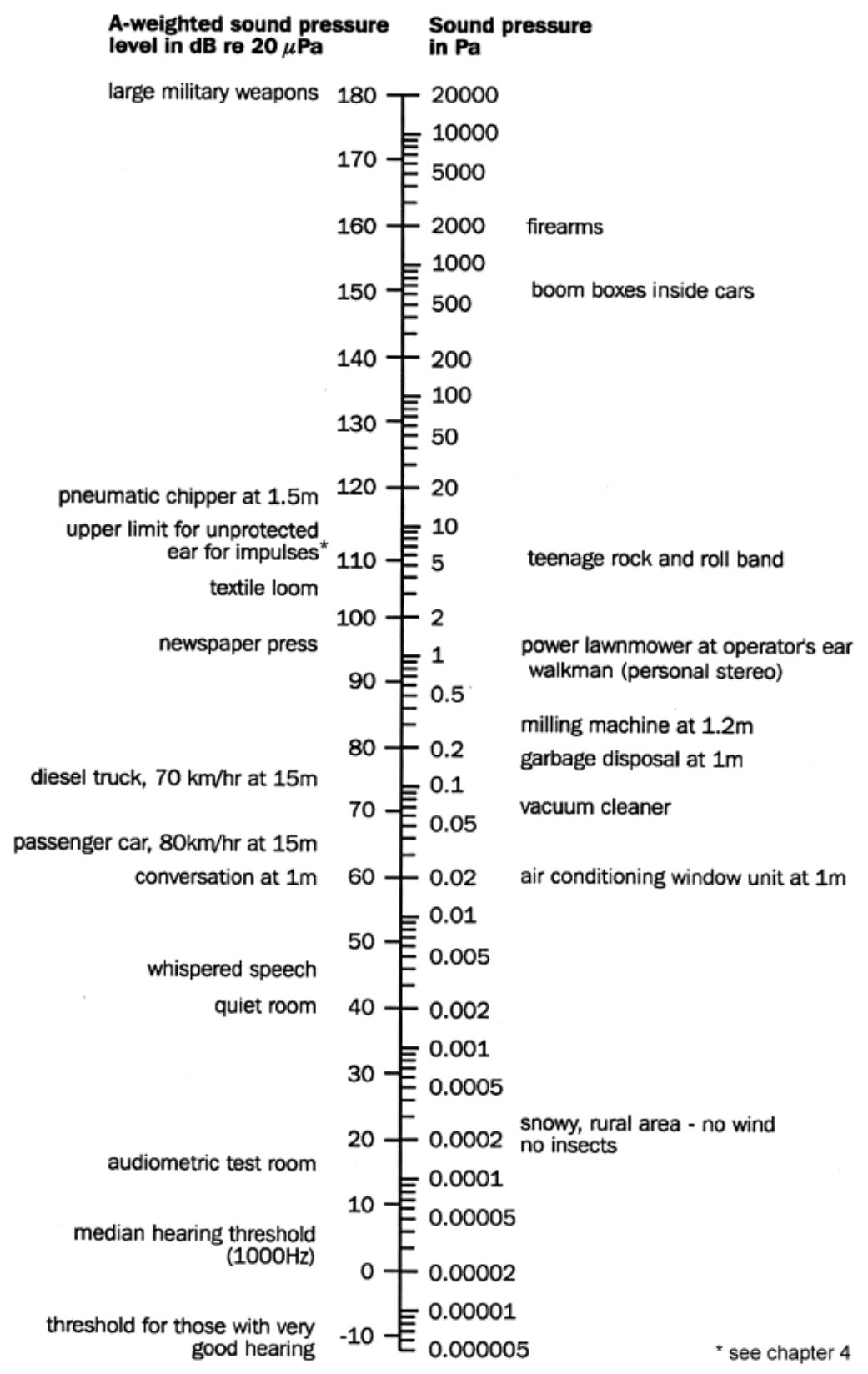




\section{Appendix B \\ MATLAB Script}

This MATLAB script was created to post-process the collected experimental data.

\section{Magna Testing Results}

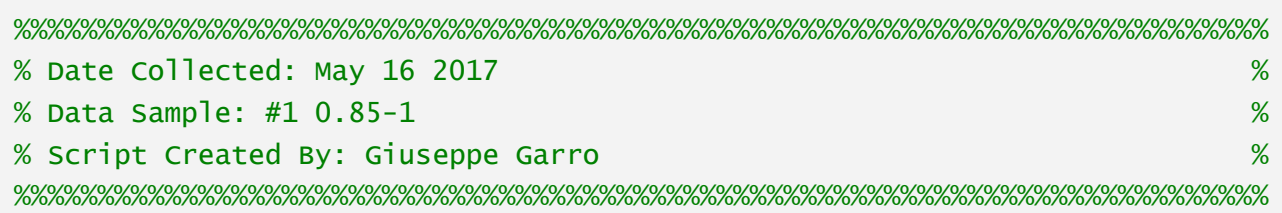

\section{Clear Stored Data \& Line Colour}

clc;

clear a11;

close a11;

\% Line Colour

$B=[0,0.4470,0.7410] ; \quad \%$ MP1 BLUE

$R=[0.8500,0.3250,0.0980] ; \%$ MP2 RED

$\mathrm{G}=[0.9290,0.6940,0.1250] ; \%$ MP3 GOLD

\section{Import Recorded Sound Pressure Trial}

filename = '\#1 $0.85-1$. txt' $^{\prime}$;

delimiterIn = '\t';

headerlinesIn $=49$;

(information)

Data = importdata(filename, delimiterIn, headerlinesIn);

samp_rate $=1.2207 \mathrm{E}-5$;

Fs $=81920$;

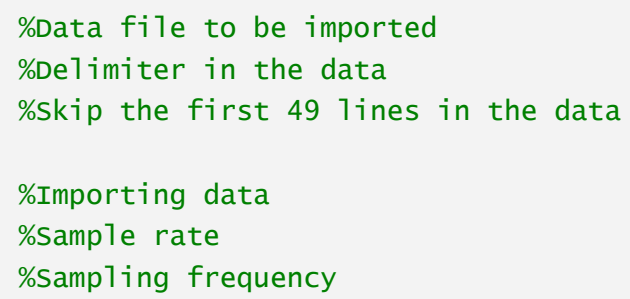

\section{Separate Data (MP1, MP2, MP3)}

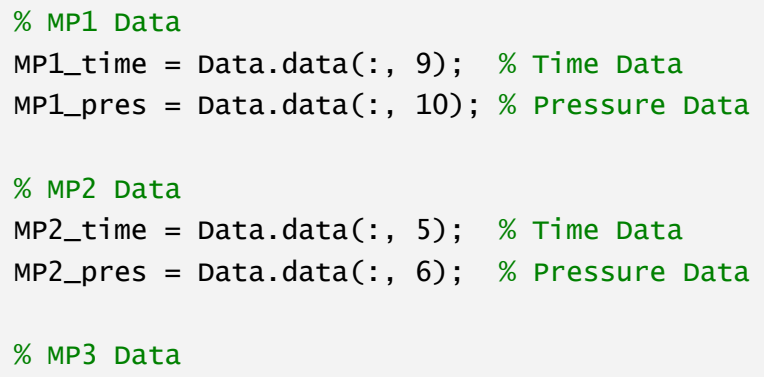


MP3_time = Data.data $(:, 1) ; \%$ Time Data

MP3_pres $=$ Data.data $(:, 2) ; \%$ Pressure Data

\section{Determine Truncating Locations}

\% step sizes used for a11 Microphone Positions

pre_max $=0 ; \quad \%$ Pre $\max$ pressure point step initialized

post_max $=0 ; \%$ Post $\max$ pressure point step initialized

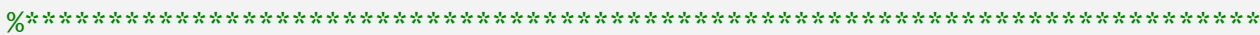

$\%$ MP1

[M_MP1, I_MP1] $=\max (\operatorname{Data} \cdot \operatorname{data}(:, 10)) ; \%$ Locate the index of the max pressure (I) and state the max pressure (M)

preN $=2621$;

\% start index serching just after latch activation

postN $=6000$;

$\%$ start index serching close to end of sound decay

\% PRE Latch Activation

\% Initial points used to calculate the slope at 5 positions $(1,2,3,10,15$ samples) before pren

\% Absolute slope 1

pre_x1_1 = Data.data ((I_MP1-preN) $-($ pre_max $), 9)$;

pre_y1_1 = Data.data ((I_MP1-preN) $-($ pre_max $), 10)$;

pre_x2_1 = Data.data ((I_MP1-preN) $-($ pre_max+1), 9);

pre_y2_1 = Data.data ((I_MP1-preN) $-($ pre_max+1), 10);

pre_abs_s1ope_1 = abs $(($ pre_y2_1-pre_y1_1)./(pre_x2_1-pre_x1_1));

\% Absolute slope 2

pre_x1_2 = Data.data((I_MP1-pren-1)-(pre_max), 9);

pre_y1_2 = Data.data ((I_MP1-preN-1)-(pre_max), 10);

pre_x2_2 = Data.data ((I_MP1-preN-1)-(pre_max+1), 9);

pre_y2_2 = Data.data ((I_MP1-preN-1)-(pre_max+1), 10);

pre_abs_s1ope_2 = abs (

$\%$ Absolute slope 3

pre_x1_3 = Data.data ((I_MP1-preN-2)-(pre_max $), 9)$;

pre_y1_3 = Data.data ((I_MP1-preN-2)-(pre_max), 10);

pre_x2_3 = Data.data ((I_MP1-preN-2) $-($ pre_max+1), 9);

pre_y2_3 = Data.data ((I_MP1-preN-2)- $($ pre_max+1), 10);

pre_abs_s1ope_3 = abs ( pre_y2_3-pre_y1_3)./(pre_x2_3-pre_x1_3));

\% Absolute slope 4

pre_x1_4 = Data.data ((I_MP1-preN-10)-(pre_max $), 9)$;

pre_y1_4 = Data.data ( I_MP1-preN-10)-(pre_max $), 10)$;

pre_x2_4 = Data.data ((I_MP1-preN-10)-(pre_max+1), 9);

pre_y2_4 = Data.data ( I_MP1-preN-10)- $($ pre_max+1), 10);

pre_abs_s1ope_4 = abs (

\% Absolute slope 5

pre_x1_5 = Data.data((I_MP1-preN-15)-(pre_max), 9);

pre_y1_5 = Data.data ( I_MP1-preN-15)-(pre_max), 10);

pre_x2_5 = Data.data ((I_MP1-preN-15)-(pre_max+1), 9);

pre_y2_5 = Data.data ( I_MP1-preN-15)- $($ pre_max +1$), 10)$;

pre_abs_s1ope_5 = abs $(($ pre_y2_5-pre_y1_5 $) . /($ pre_x2_5-pre_x1_5) );

\%\%\%\%\%\%\%\%\%\%\%\%\%\%\%\%\%\%\%\%\%\%\%\%\%\%\%\%\%\%\%\%\%\%\%\%\%\%\%\%\%\%\%\%\%\%\%\%\%\%\%\%\%\%\%\%\%\%\%

\% Loop "pre max" pressure point to find index/pressure right before \% 
\% 1atch/striker impact using slope between 2 points. If the absolute slope \% $\%$ is below 150 at the 5 calculated points, it means we are at ambient sound\% $\%$ pressure just before latch activation. If the absolute slope of at 1 east \% $\%$ one point is above 150 , the search window is shifted over to the 1 eft by $\%$

$\%$ one and the calculation repeats.

\%\%\%\%\%\%\%\%\%\%\%\%\%\%\%\%\%\%\%\%\%\%\%\%\%\%\%\%\%\%\%\%\%\%\%\%\%\%\%\%\%\%\%\%\%\%\%\%\%\%\%\%\%\%\%\%\%\%\%

while (pre_abs_slope_1 >= 150) || (pre_abs_slope_2 >= 150) || (pre_abs_slope_3 >= 150) || (pre_abs_slope_4 >=150) || (pre_abs_slope_5 >=150)

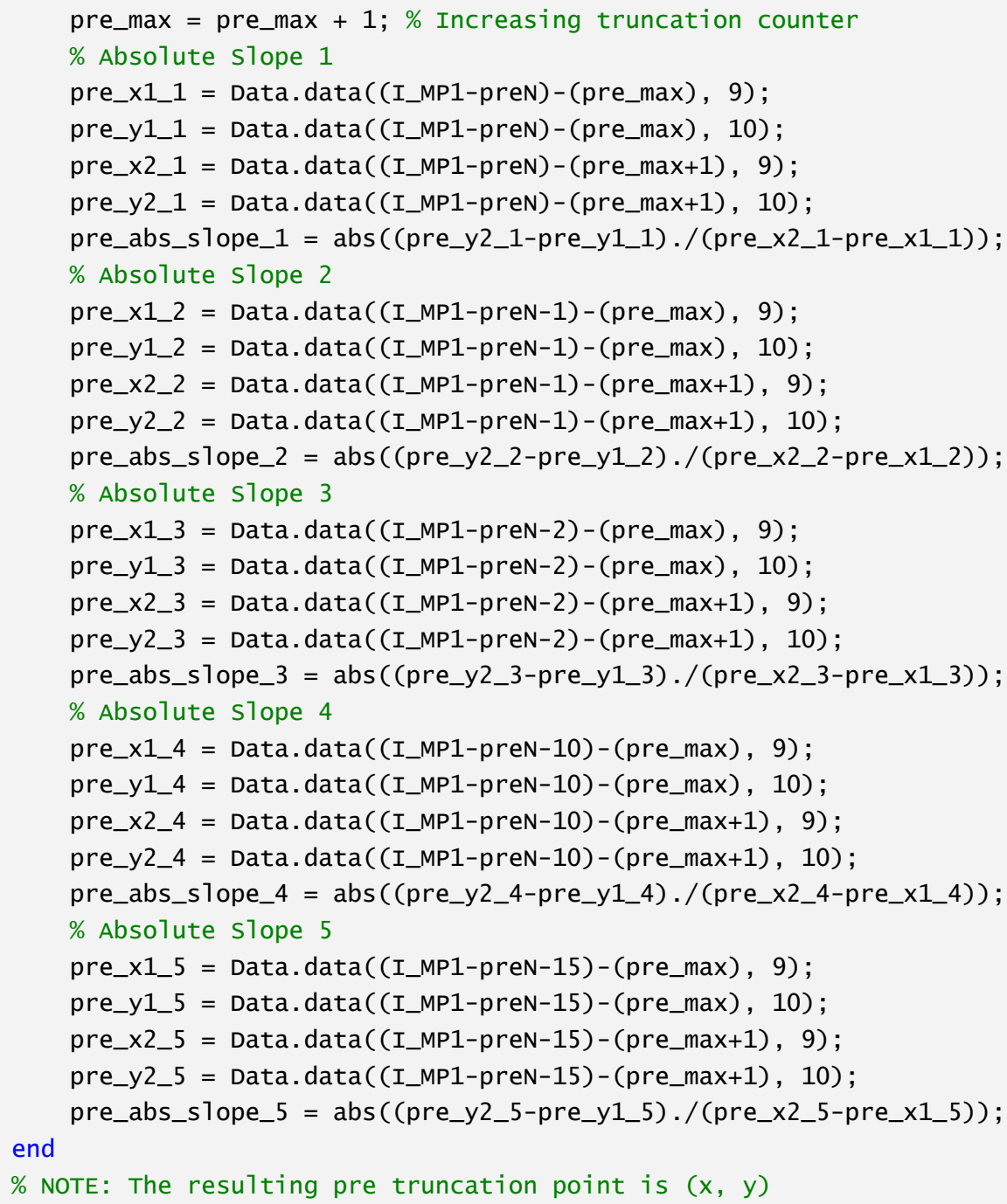




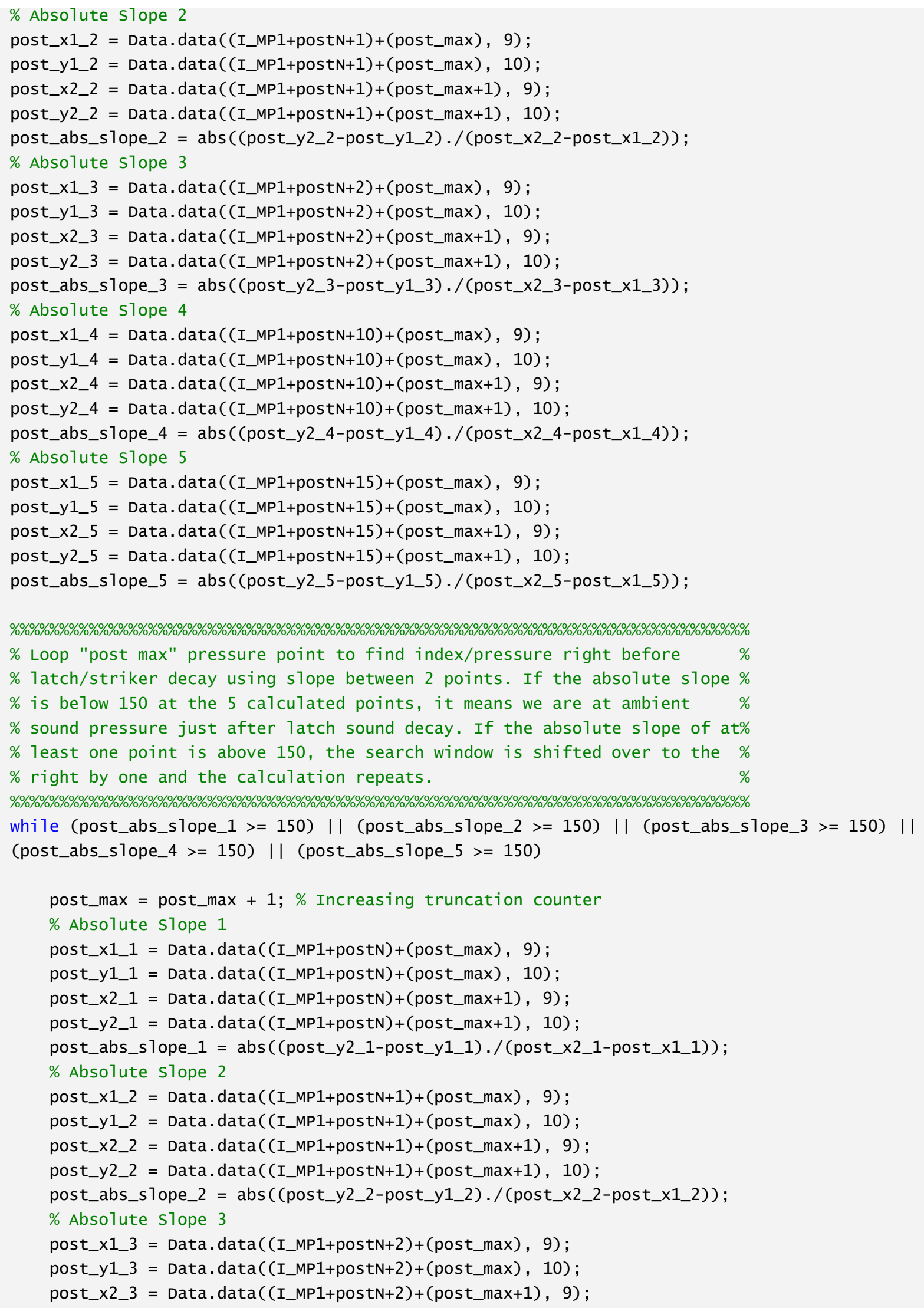


post_y2_3 = Data.data $\left(\left(I \_M P 1+p o s t N+2\right)+(\right.$ post_max+1), 10);

post_abs_s1ope_3 = abs (

\% Absolute slope 4

post_x1_4 = Data.data $\left(\left(I \_M P 1+\right.\right.$ postN+10) $+($ post_max $\left.), 9\right)$;

post_y1_4 = Data.data $\left(\left(I \_M P 1+\right.\right.$ postN+10)+(post_max $\left.), 10\right)$;

post_x2_4 = Data.data $\left(\left(I \_M P 1+\right.\right.$ postN+10) $+($ post_max +1$\left.), 9\right)$;

post_y2_4 = Data.data $\left(\left(I \_M P 1+\right.\right.$ postN +10$)+($ post_max +1$\left.), 10\right)$;

post_abs_s1ope_4 = abs $($ post_y2_4-post_y1_4) $/($ post_x2_4-post_x1_4) $)$;

\% Absolute slope 5

post_x1_5 = Data.data ((I_MP1+postN+15)+(post_max), 9);

post_y1_5 = Data.data ( I_MP1+postN+15)+(post_max $), 10)$;

post_x2_5 = Data.data $\left(\left(I \_M P 1+p o s t N+15\right)+(\right.$ post_max+1), 9);

post_y2_5 = Data.data $(($ I_MP1+postN+15) $+($ post_max+1), 10);

end

post_abs_s1ope_5 = abs (

\% NOTE: The resulting post truncation point is after loop is ( $x, y)$

fprintf('MP1:The Pressure and Time right before impact is \%7.6f Pa and \%7.6f seconds. RED MARKER \n', Data.data((I_MP1-preN)-(pre_max), 10), Data.data((I_MP1-preN)-(pre_max), 9)); fprintf('MP1:The Pressure and Time right after residual decay is \%7.6f Pa and \%7.6f seconds. GREEN MARKER $\backslash n$ ', Data.data((I_MP1+postN)+(post_max), 10), Data.data((I_MP1+postN)+(post_max), 9));

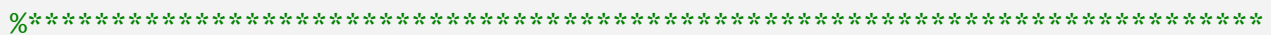

$\%$ MP2

\% (Uses PRE and POST truncation points from MP1)(To be consistent, MP1 max index point was used for truncation. Results in same number of data points as MP1)

[M_MP2, I_MP2] $=\max (\operatorname{Data} \cdot \operatorname{data}(:, 6)) ; \%$ Locate the index of the max pressure (I) and state the max pressure (M)

fprintf('MP2:The Pressure and Time right before impact is \%7.6f Pa and \%7.6f seconds. RED MARKER \n', Data.data((I_MP1-preN)-(pre_max), 6), Data.data((I_MP1-preN)-(pre_max), 5)); fprintf('MP2:The Pressure and Time right after residual decay is \%7.6f Pa and \%7.6f seconds. GREEN MARKER $\backslash n$ ', Data.data((I_MP1+postN)+(post_max), 6), Data.data((I_MP1+postN)+(post_max), 5));

$\%$ MP3

\% (Uses PRE and POST truncation points from MP1)(To be consistent, MP1 max index point was used for truncation. Results in same number of data points as MP1)

[M_MP3, I_MP3] $=\max (\operatorname{Data} \cdot \operatorname{data}(:, 2)) ; \%$ Locate the index of the max pressure (I) and state the max pressure $(M)$

fprintf('MP3:The Pressure and Time right before impact is \%7.6f Pa and \%7.6f seconds. RED MARKER \n', Data.data((I_MP1-preN)-(pre_max), 2), Data.data((I_MP1-preN)-(pre_max), 1)); fprintf('MP3:The Pressure and Time right after residual decay is \%7.6f Pa and \%7.6f seconds. GREEN MARKER $\backslash n^{\prime}$, Data.data((I_MP1+postN)+(post_max), 2), Data.data((I_MP1+postN)+(post_max), 1));

\section{Plotting Raw Data In A Single Figure}

\%\%\%\%\%\%\%\%\%\%\%\%\%

$\%$ NOT Truncated \%

\%\%\%\%\%\%\%\%\%\%\%\%\% 
\%Figure Number ( $1 / 11)$

$\%$ Plot of Time vs. Sound Pressure $[\mathrm{Pa}]$

\% (To be consistent MP1 max index point used for truncation. Results in same number of data points for MP1, MP2, MP3)

$\%$ subplot

figure('Name', strrep(filename, '.txt', ''));

$\%$ MP1

$\operatorname{subplot}(3,1,1)$

plot(MP1_time, MP1_pres, 'Color', B)

title('MP1')

xlabel ('Time [s]')

ylabel ('Pressure $[\mathrm{Pa}]$ ')

axis auto

hold on

plot(Data.data((I_MP1-preN)-(pre_max), 9), Data.data((I_MP1-preN)-(pre_max), 10), 'marker', '*', 'color', 'c', 'markersize', 15, '1inewidth', 2)

plot (Data.data ((I_MP1+postN)+(post_max), 9), Data.data((I_MP1+postN)+(post_max), 10), 'marker', '*', 'color', 'm', 'markersize', 15, '1inewidth', 2)

hold off

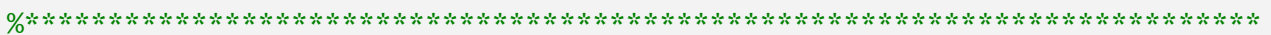

\% MP2

subplot $(3,1,2)$

plot(MP2_time, MP2_pres, 'Color', R);

title ('MP2')

xlabel ('Time $[\mathrm{s}]$ ')

ylabel ('Pressure $[\mathrm{Pa}]^{\prime}$ )

axis auto

hold on

plot (Data.data((I_MP1-preN)-(pre_max), 5), Data.data((I_MP1-preN)-(pre_max), 6), 'marker', '*', 'color', 'c', 'markersize', 15, '1inewidth', 2)

plot (Data.data ((I_MP1+postN)+(post_max), 5), Data.data((I_MP1+postN)+(post_max), 6), 'marker' , '*', 'color', 'm', 'markersize', 15, '7inewidth', 2)

hold off

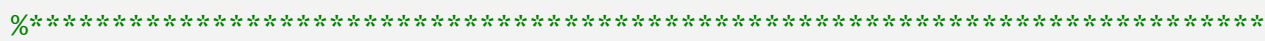

$\%$ MP3

subplot (3, 1, 3)

plot(MP3_time, MP3_pres, 'Color', G);

title('MP3')

xlabel ('Time $[\mathrm{s}]$ ')

ylabel ('Pressure $[\mathrm{Pa}]$ ')

axis auto

hold on

plot (Data.data((I_MP1-preN)-(pre_max), 1), Data.data((I_MP1-preN)-(pre_max), 2), 'marker', '*', 'color', 'c', 'markersize', 15, '1inewidth', 2)

plot (Data.data ((I_MP1+postN)+(post_max), 1), Data.data((I_MP1+postN)+(post_max), 2), 'marker' , '*', 'color', 'm', 'markersize', 15, '1inewidth', 2)

hold off

\% subplot supertitle

s1 = 'Latch ';

s2 = strrep(filename, '.txt', '');

plotT $=[s 1, s 2]$;

suptitle(plotT) 


\section{Plotting All Pressure Data In A Single Figure}

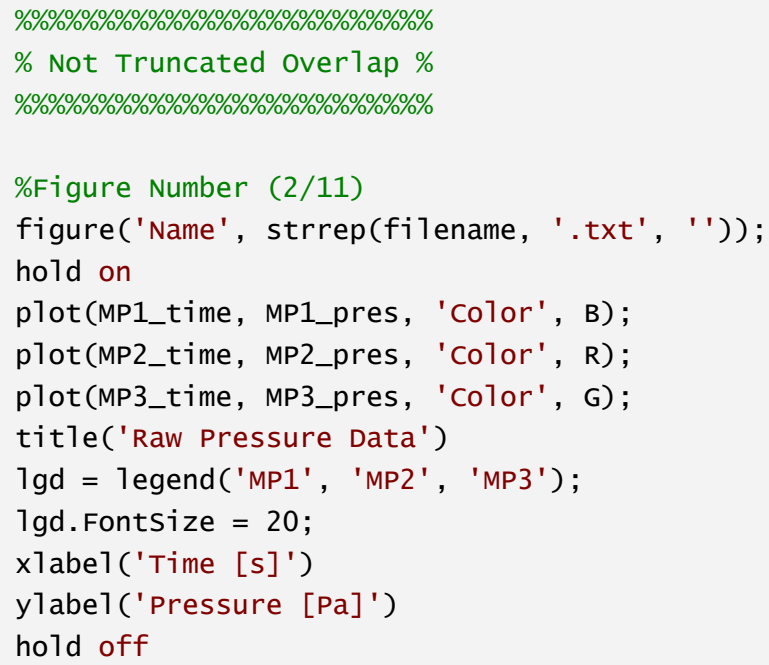

\section{Truncate Data and Place In Storage Array}

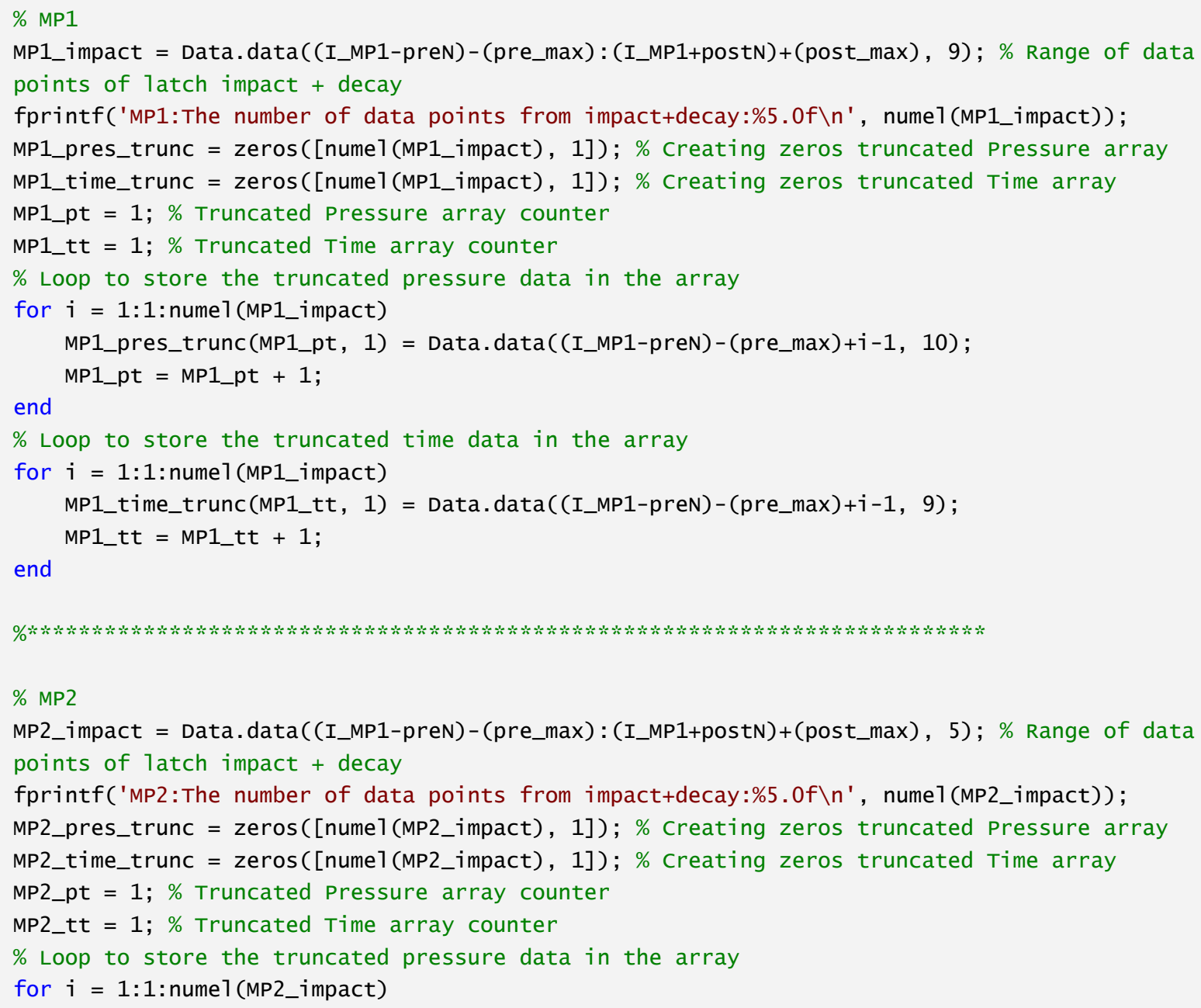




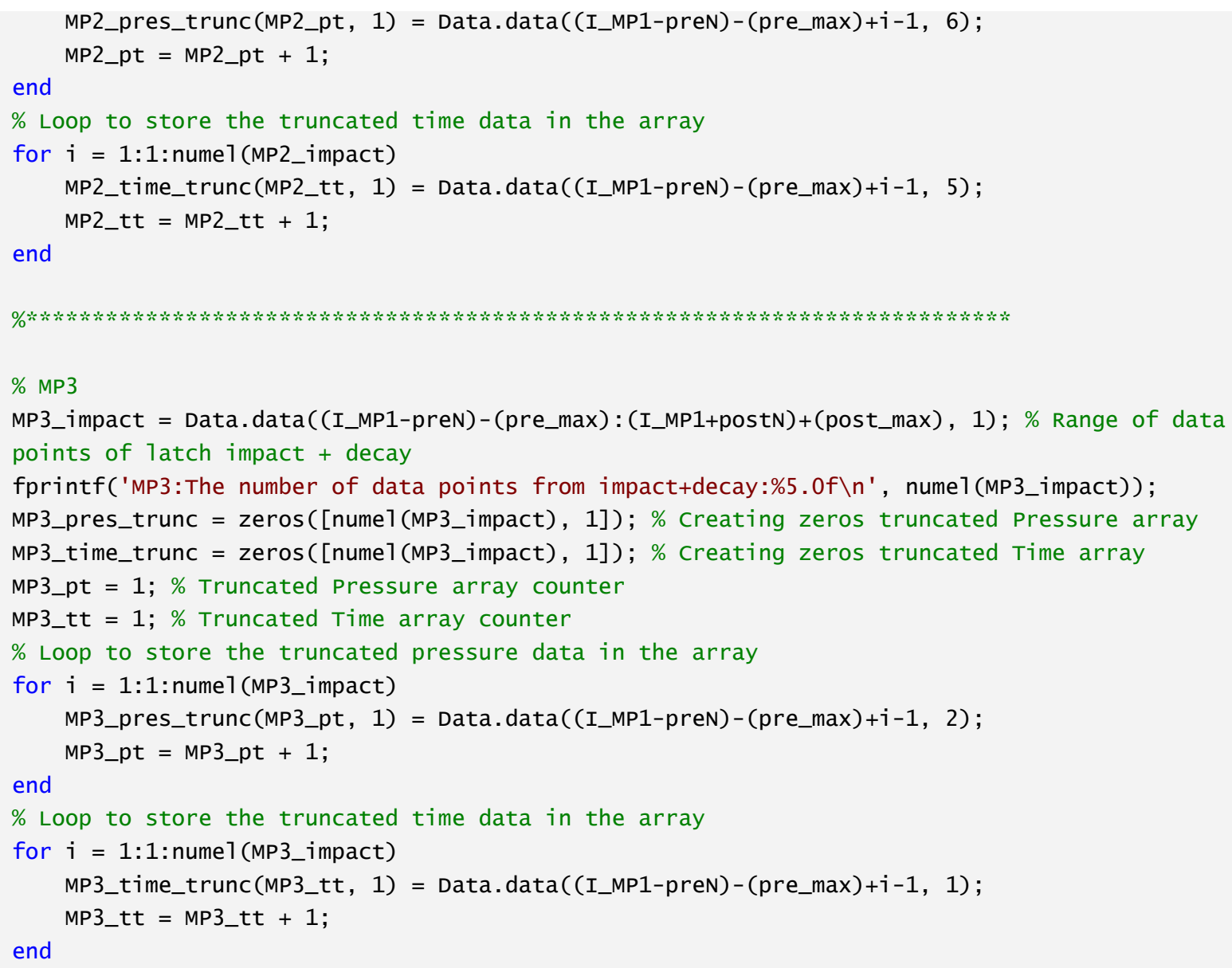

\section{Plotting Raw Data In A Single Figure}

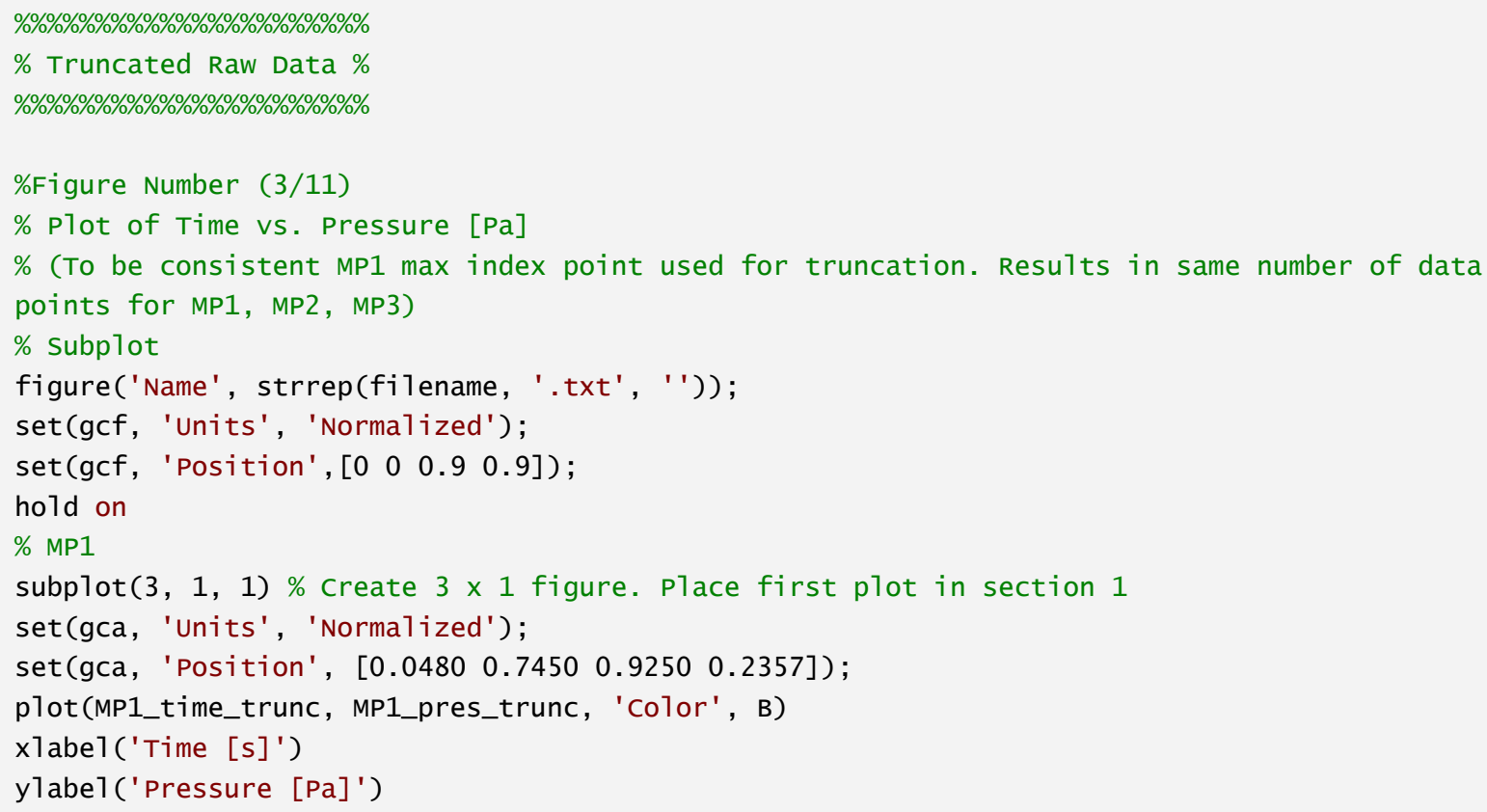


$y \lim \left(\left[\begin{array}{ll}-1.3 & 1.3\end{array}\right]\right)$;

set (gca, 'XMinorTick', 'on')

set(gca, 'YMinorTick', 'on')

axis tight

a_MP = gca;

a_MP.Fontsize $=17$;

a_MP.TitleFontSizeMultiplier $=1.1$;

a_MP.Labe1FontSizeMu1tiplier = 1.1;

legend ('MP1');

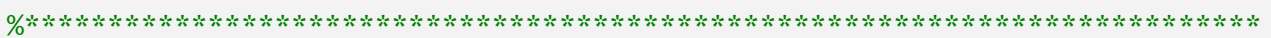

$\%$ MP2

subplot $(3,1,2) \%$ create $3 \times 1$ figure. Place first plot in section 2

set(gca, 'Units', 'Norma1ized');

set(gca, 'Position', [ $\left.\begin{array}{llll}0.0480 & 0.4196 & 0.9250 & 0.2357\end{array}\right]$ );

p1ot(MP2_time_trunc, MP2_pres_trunc, 'Color', R);

xlabel ('Time [s]')

ylabel ('Pressure $[\mathrm{Pa}]$ ')

ylim ([-1.3 1.3]);

set(gca, 'XMinorTick', 'on')

set (gca, 'YMinorTick', 'on')

axis tight

a_MP = gca;

a_MP.FontSize $=17$;

a_MP.TitleFontSizeMultiplier $=1.1$;

a_MP.Labe1FontSizeMu1tiplier = 1.1;

1egend ('MP2') ;

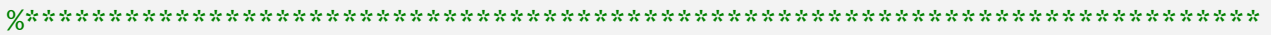

$\%$ MP3

subplot $(3,1,3) \%$ create $3 \times 1$ figure. Place first plot in section 3

set(gca, 'Units', 'Normalized');

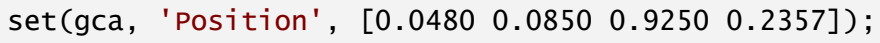

plot(MP3_time_trunc, MP3_pres_trunc, 'Color', G);

xlabel ('Time [s]')

ylabel ('Pressure $[\mathrm{Pa}]$ ')

ylim ([-1.3 1.3]);

set (gca, 'XMinorTick', 'on')

set(gca, 'YMinorTick', 'on')

axis tight

a_MP = gca;

a_MP. FontSize $=17$

a_MP.TitleFontSizemultiplier $=1.1$;

a_MP.Labe1FontSizemu1tiplier $=1.1$;

legend ('MP3');

hold off

\section{Plot Overlaid Truncated Raw Pressure Data}

\%\%\%\%\%\%\%\%\%\%\%\%\%\%\%

\% Truncated overlap \%

\%\%\%\%\%\%\%\%\%\%\%\%\%\%\% 


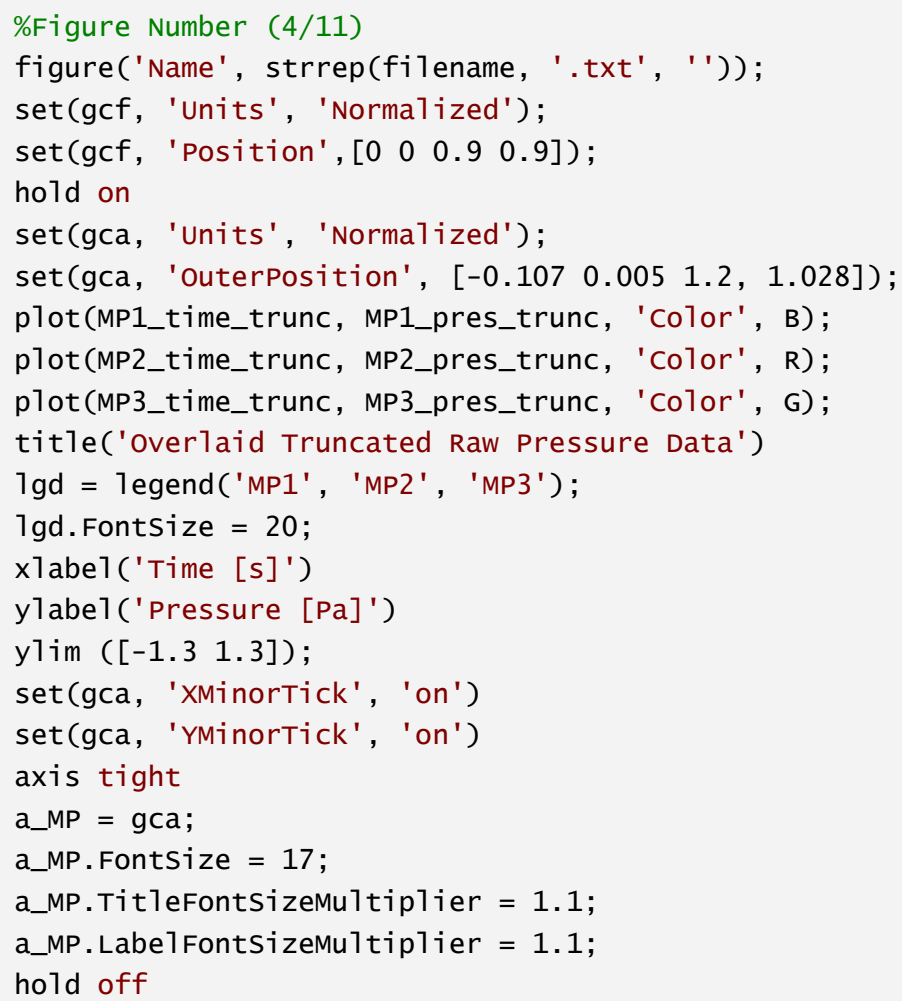

\section{A-Weighting Filter [DBA]}

\section{\%\%\%\%\%\%\%\%\%\%\%\%\%\%\%\%\%\%\%\%\%\%\%\%\%\%\%\%\%\%\%\%\%\%\%\%\%\%\%\%\%\%\%\%\%\%\%\%\%\%\%\%\%\%\%\%}

\% A-Weighting Filter functions created by Dr. Christophe Couvreur. \% $\%$ These functions are not originally mine but have been altered for the \% $\%$ purpose of this research. Scripts downloaded from Matlab File Exchange \% 


\section{1/3 Octave Band Plot}

\%\%\%\%\%\%\%\%\%\%\%\%\%\%\%\%\%\%\%\%\%\%\%\%\%\%\%\%\%\%\%\%\%\%\%\%\%\%\%\%\%\%\%\%\%\%\%\%\%\%\%\%\%\%\%\%\%\%\%\%

\% 1/3 octave Band Plot functions created by Dr. Christophe Couvreur. \%

$\%$ These functions are not originally mine but have been altered for the \%

\% purpose of this research. Scripts downloaded from Matlab File Exchange \%

\% [https://www.mathworks.com/mat1abcentra1/fileexchange/69-octave] \% \%\%\%\%\%\%\%\%\%\%\%\%\%\%\%\%\%\%\%\%\%\%\%\%\%\%\%\%\%\%\%\%\%\%\%\%\%\%\%\%\%\%\%\%\%\%\%\%\%\%\%\%\%\%\%\%\%\%\%\%

\% creating a Filter Bank from 20-20kHz

[P_MP1, F_MP1] = fi1tbank(MP1_pres_trunc, Fs, [], 'extended'); \%MP1 Fi1terbank

[P_MP2, F_MP2] = filtbank(MP2_pres_trunc, Fs, [], 'extended'); \%MP2 Fi1terbank

[P_MP3, F_MP3] = fi1tbank (MP3_pres_trunc, Fs, [], 'extended'); \%MP3 Fi1terbank

\% Applying the Fi1terbank to MP1, MP2, MP3

\% MP1 (5/11)

figure('Name', strrep(filename, '.txt', ''));

h_MP1 = bankdisp (P_MP1, F_MP1);

tit1e('MP1')

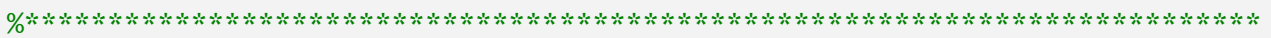

\% MP2 (6/11)

figure('Name', strrep(filename, '.txt', ''));

h_MP2 = bankdisp(P_MP2, F_MP2);

title('MP2')

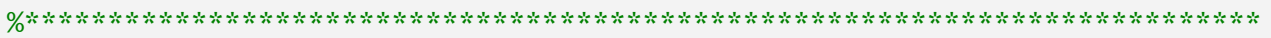

\% MP3 $(7 / 11)$

figure('Name', strrep(filename, '.txt', ''));

h_MP3 = bankdisp(P_MP3, F_MP3);

title('MP3')

\% Inverting ASPL

P_MP1 = P_MP1';

P_MP2 = P_MP2';

P_MP3 = P_MP3' ;

\section{Combine All Three 1/3 Octave Band Plots Into One}

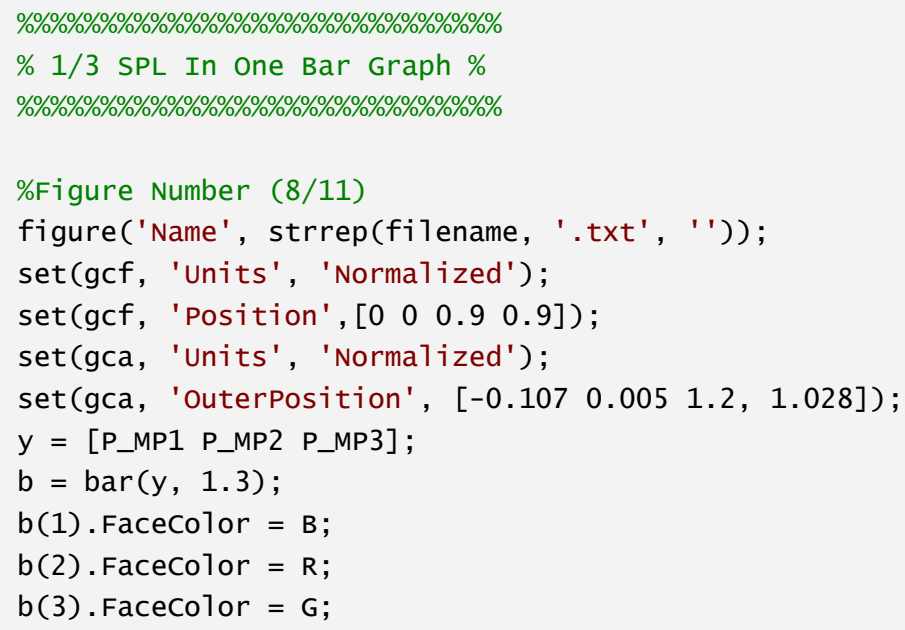


$1 \mathrm{gd}=1$ egend ('MP1', 'MP2', 'MP3', 'Location', 'northwest');

title('SPL vs. 1/3 Octave Band frequency')

1gd. FontSize $=20$;

$\mathrm{x}$ label ('Frequency Band $[\mathrm{Hz}]$ ')

ylabel ('Sound Pressure Leve1 [dBA]')

set(gca, 'YMinorTick', 'on')

a_bar = gca;

a_bar.FontSize $=17$;

a_bar.TitleFontSizemultiplier $=1.1$;

a_bar.Labe1FontSizeMu1tiplier $=1.1$;

a_bar.XLim = ([ $\left.\left[\begin{array}{ll}0 & 31\end{array}\right]\right)$;

a_bar.XTick = $(1: 1: 30)$;

a_bar.XTickLabe1 = (\{'25';'32';'40';'50';'63';'80';'100';'125';'160'; ...

'200'; '250'; '315'; '400'; '500'; '630'; '800'; '1000'; ... .

'1250';'1600';'2000';'2500';'3150'; '4000';'5000'; ...

'6300'; '8000'; '10000'; '12500'; '16000' ; '20000'\});

a_bar.XTickLabe1Rotation $=29$;

\section{Continuous Wavelet Transform (CWT) Analysis}

\%\%\%\%\%\%\%\%\%\%\%\%\%\%\%\%\%\%\%\%\%\%\%\%\%\%\%\%\%\%\%\%\%\%\%\%\%\%\%\%\%\%\%\%\%\%\%\%\%\%\%\%\%\%\%\%\%\%\%\%

$\%$ Plots the magnitude scalogram of the recorded sound pressure data as a $\%$

$\%$ function of time.

\%\%\%\%\%\%\%\%\%\%\%\%\%\%\%\%\%\%\%\%\%\%\%\%\%\%\%\%\%\%\%\%\%\%\%\%\%\%\%\%\%\%\%\%\%\%\%\%\%\%\%\%\%\%\%\%\%\%\%\%

\% MP1 Pressure and CWT (9/11)

figure ('Name', strrep(filename, '.txt', ''));

set(gcf, 'Units', 'Normalized');

set(gcf, 'Position', [ [ [ $\left.\begin{array}{llll}0 & 0 & 0.9 & 0.9\end{array}\right]$ );

hold on

subplot $(2,1,1)$;

set(gca, 'Units', 'Normalized');

set(gca, 'Position', [ [ $\left.\begin{array}{llll}0.050 & 0.54 & 0.878 & 0.420\end{array}\right]$ );

plot(MP1_time_trunc, MP1_pres_trunc, 'Color', B)

title('MP1: Pressure and Magnitude Scalogram')

ylabel ('Pressure [Pa]')

axis auto

set (gca, 'xMinorTick', 'on')

axis tight

a_MP = gca;

a_MP.FontSize $=17$;

a_MP.TitleFontSizeMultiplier $=1.1$;

a_MP.Labe1FontSizeMu1tip1ier $=1.1$;

a_MP.XLabe1.String = 'Time [s]';

a_MP.XLabe1.Position $=\left[\begin{array}{llll}3.6400 & -0.660 & -1.0000\end{array}\right]$;

a_MP.XLabe1.FontSize $=16.7$;

subplot $(2,1,2)$;

set(gca, 'Units', 'Normalized');

set(gca, 'Position', [ [ $\left.\begin{array}{llll}0.050 & 0.010 & 0.9160 & 0.485\end{array}\right]$ );

cwt(MP1_pres_trunc, 'morse', Fs, 'Numoctaves', 5, 'Voicesperoctave', 48) 


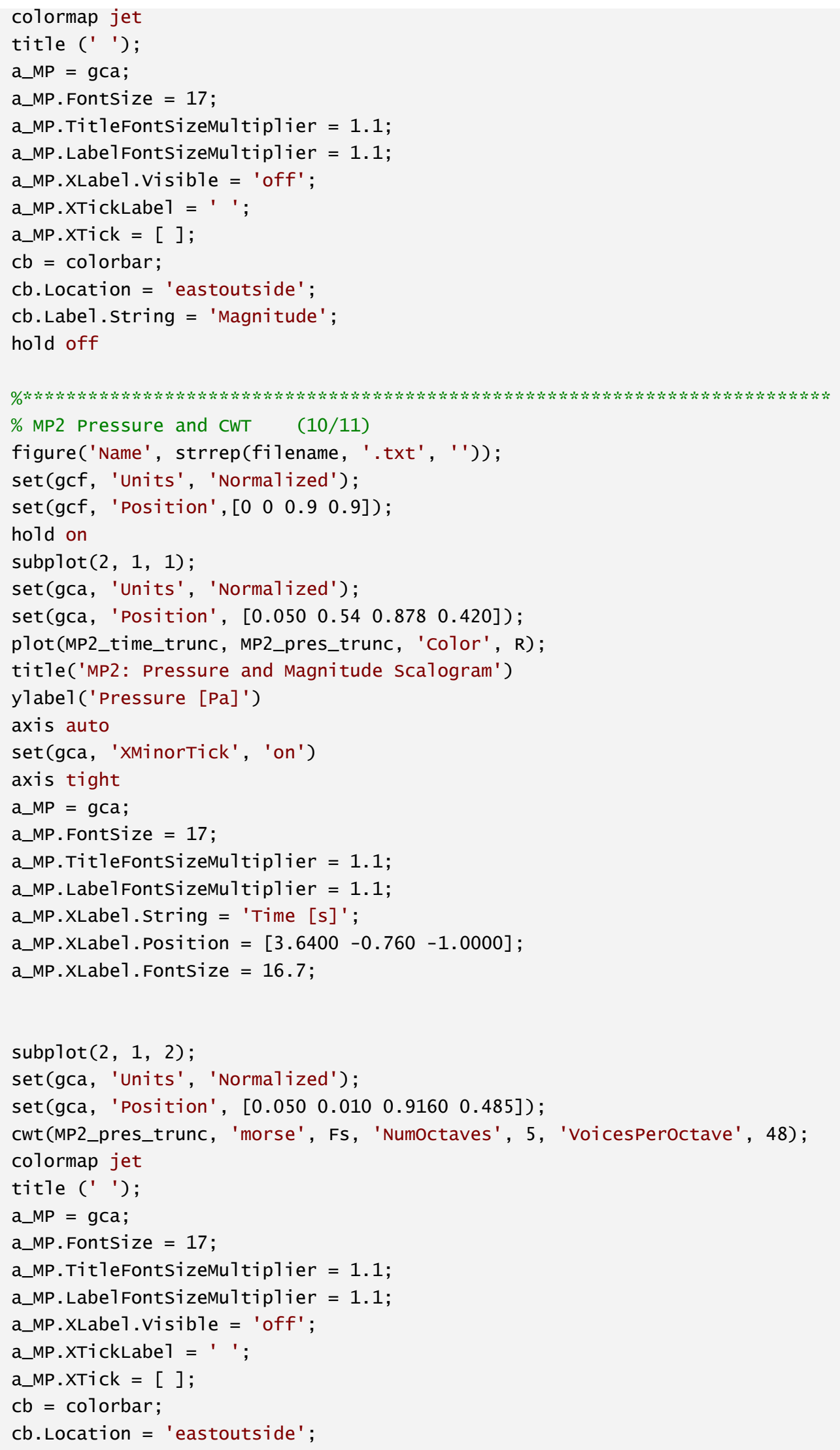




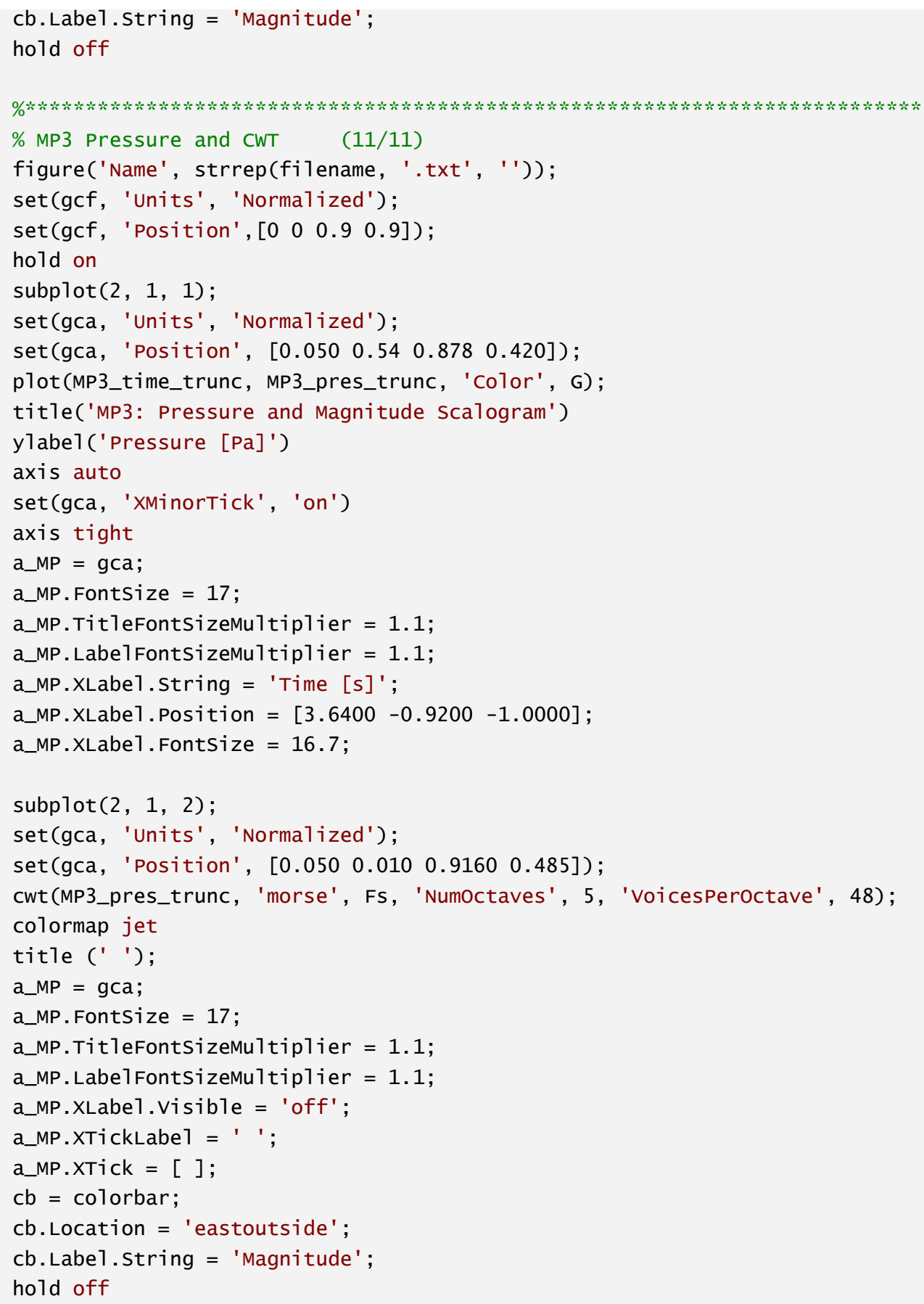




\section{Appendix C \\ MATLAB Script: Incoherent Sound Pressure Level Addition}

This MATLAB script was created to perform an incoherent SPL addition from the data generated in the MATLAB script shown in Appendix B.

\section{Magna Testing Results: Incoherent Sound Pressure Level Addition}

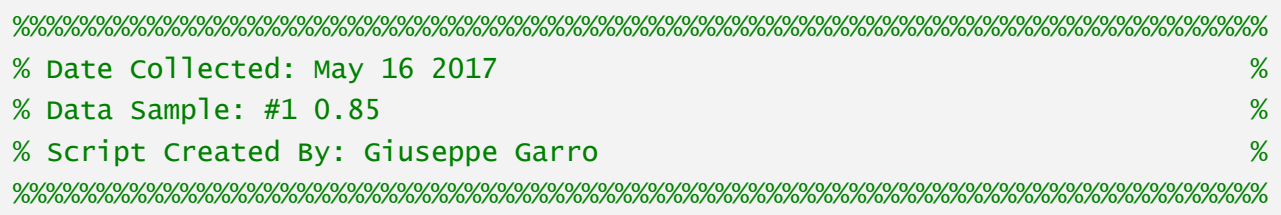

\section{Clear Stored Data, Line Colour \& Constants}

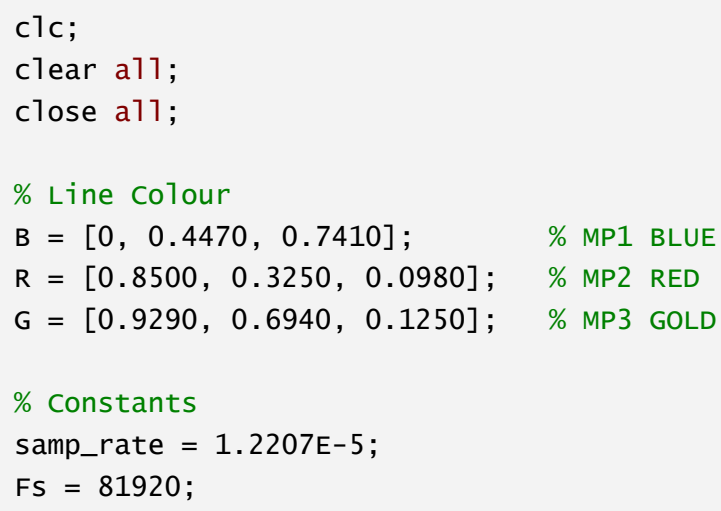

\section{Importing Sound Pressure Level Data}

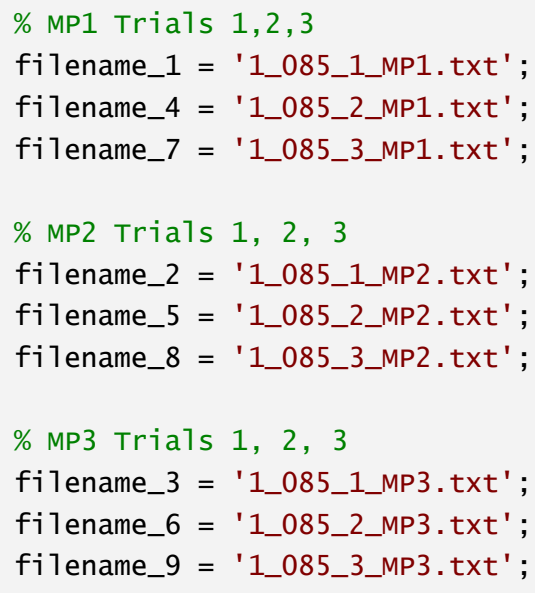




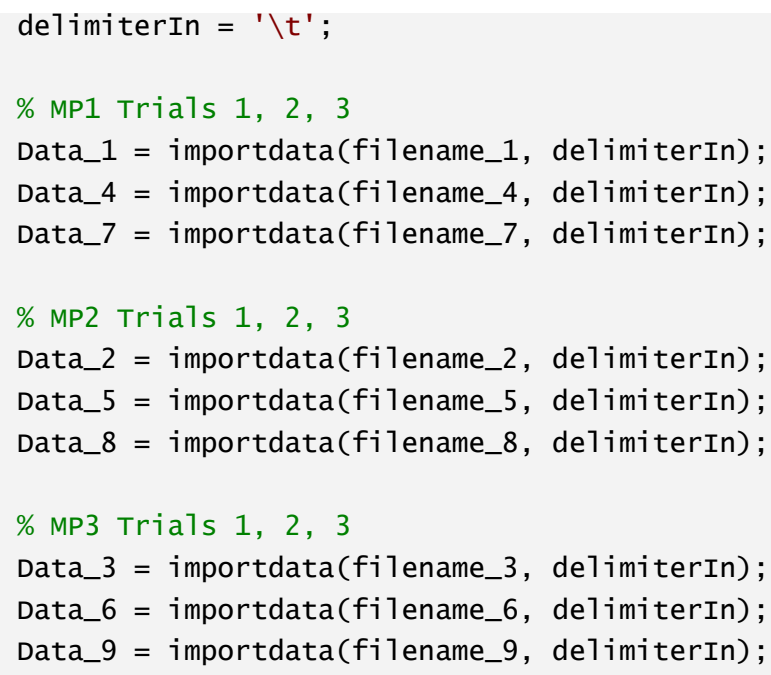

\section{Incoherent Sound Pressure Level Addition 1}

\%\%\%\%\%\%\%\%\%\%\%\%\%\%\%\%\%\%\%\%\%\%\%\%\%\%\%\%\%\%\%\%\%\%\%\%\%\%\%\%\%\%\%\%\%\%\%\%\%\%\%\%\%\%\%\%\% $\%$ Adding SPLS of MPS to obtain an Average Sound Pressure Level for each \% $\%$ separate microphone position for each trial. Average of all trials (3). \% \%\%\%\%\%\%\%\%\%\%\%\%\%\%\%\%\%\%\%\%\%\%\%\%\%\%\%\%\%\%\%\%\%\%\%\%\%\%\%\%\%\%\%\%\%\%\%\%\%\%\%\%\%\%\%\%\%

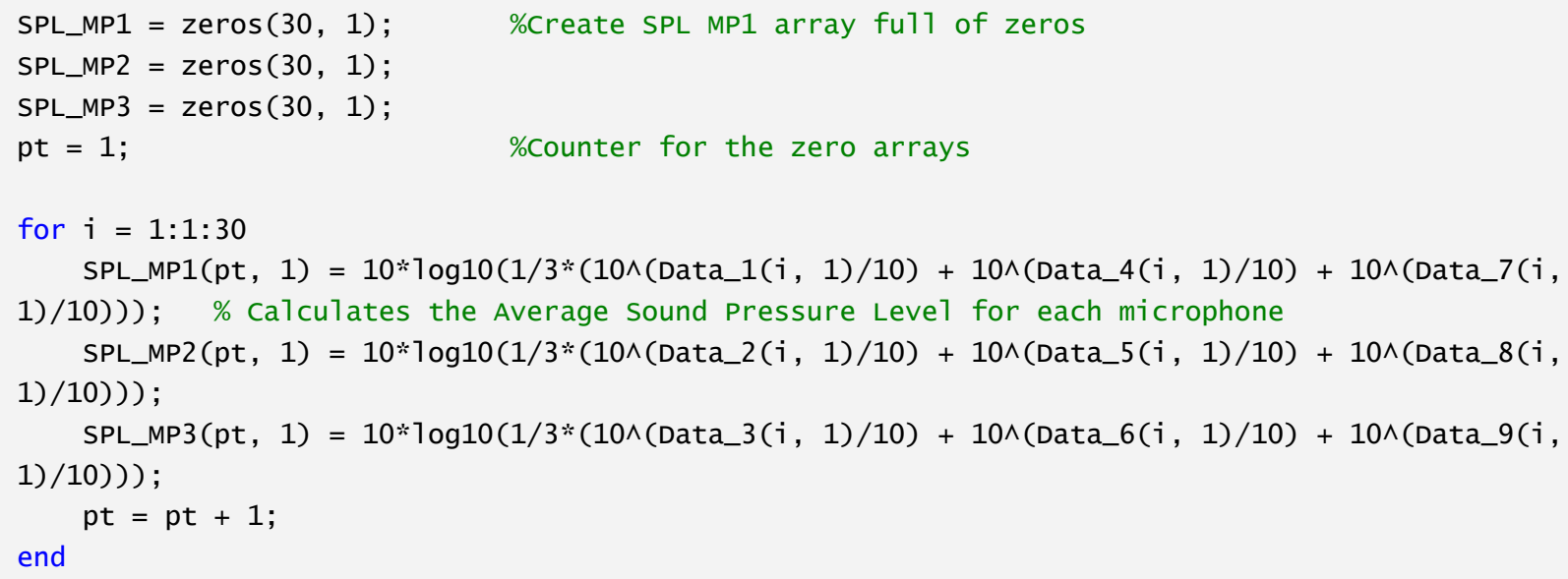

\section{Combine All Three 1/3 Octave Band Plots Into One Plot}

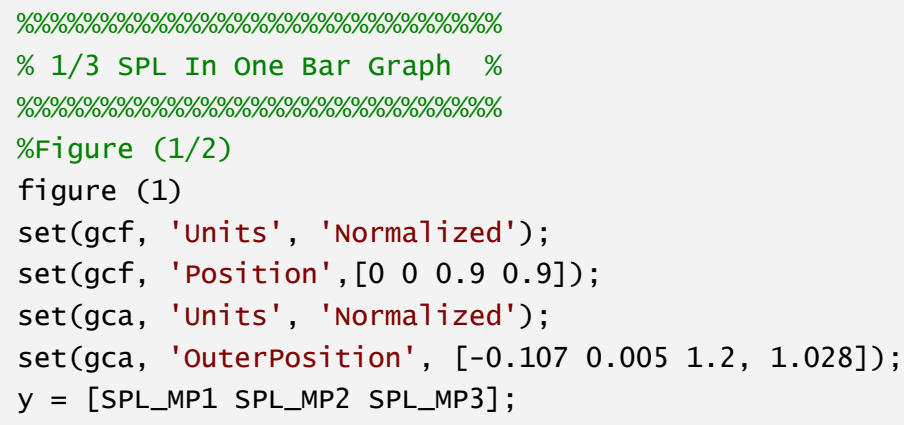




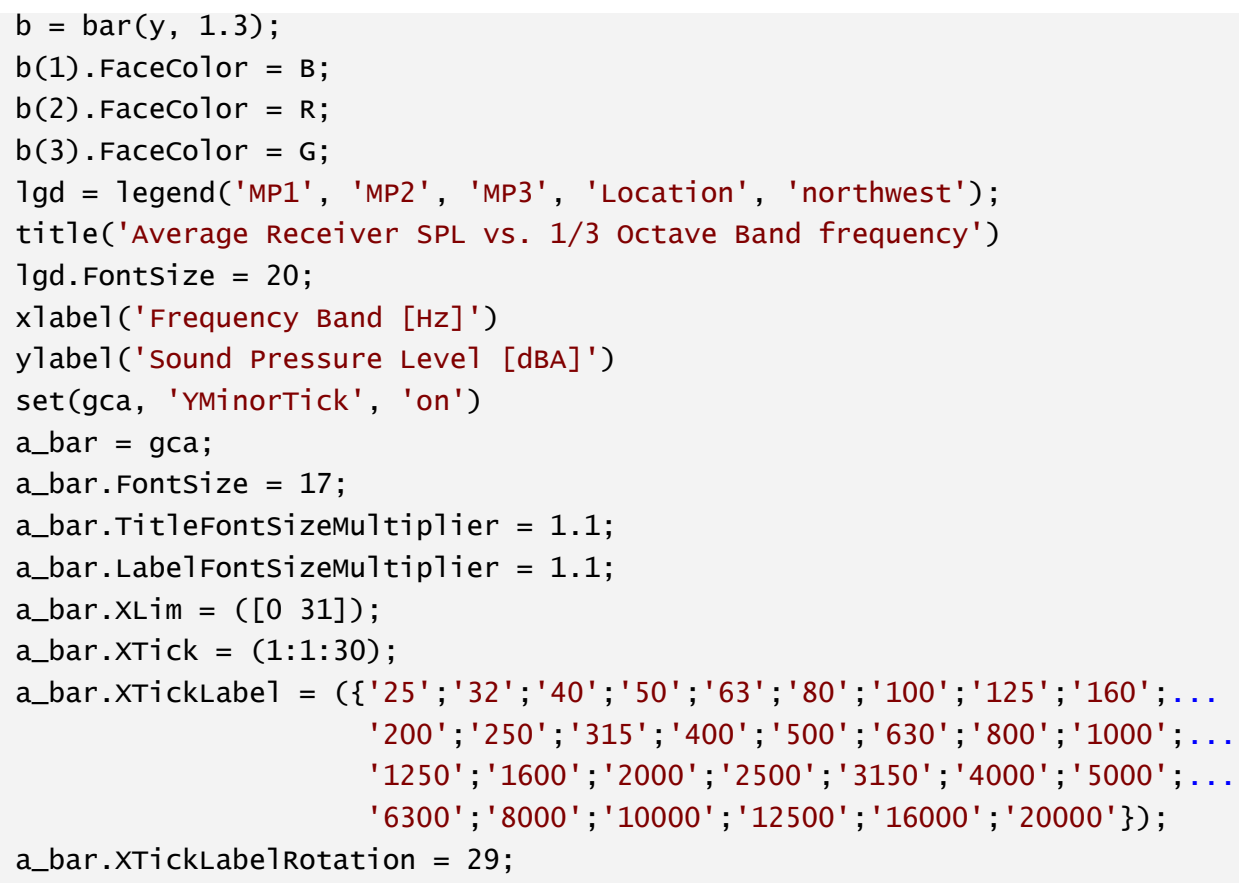

\section{Incoherent Sound Pressure Level Addition 2}

\%\%\%\%\%\%\%\%\%\%\%\%\%\%\%\%\%\%\%\%\%\%\%\%\%\%\%\%\%\%\%\%\%\%\%\%\%\%\%\%\%\%\%\%\%\%\%\%\%\%\%\%\%\%\%\%\%\%\%\% $\%$ Adding SPLS of MPS to obtain an Average Sound Pressure Level of al1 the \% $\%$ microphone position for each trial. Average of all microphone positions \%

$\%$ (3) and all trials (3).

$\operatorname{ASPL}=\operatorname{zeros}(30,1)$

pt $=1$;

for $i=1: 1: 30$

$\operatorname{ASPL}(p t, 1)=10 * \log 10\left(1 / 3 *\left(10 \wedge\left(\operatorname{SPL} \_M P 1(i, 1) / 10\right)+10 \wedge\left(S P L \_M P 2(i, 1) / 10\right)+10 \wedge\left(S P L \_M P 3(i\right.\right.\right.$, 1)/10))); \% calculates the Average Sound Pressure Level for Entire Sound Field measured by each microphone $p t=p t+1 ;$

end

\section{Average Sound Pressure Level vs. 1/3 Octave Bandwidth}

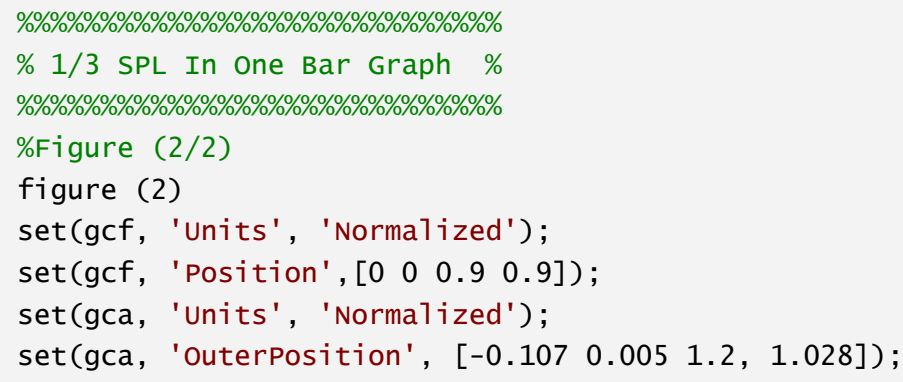




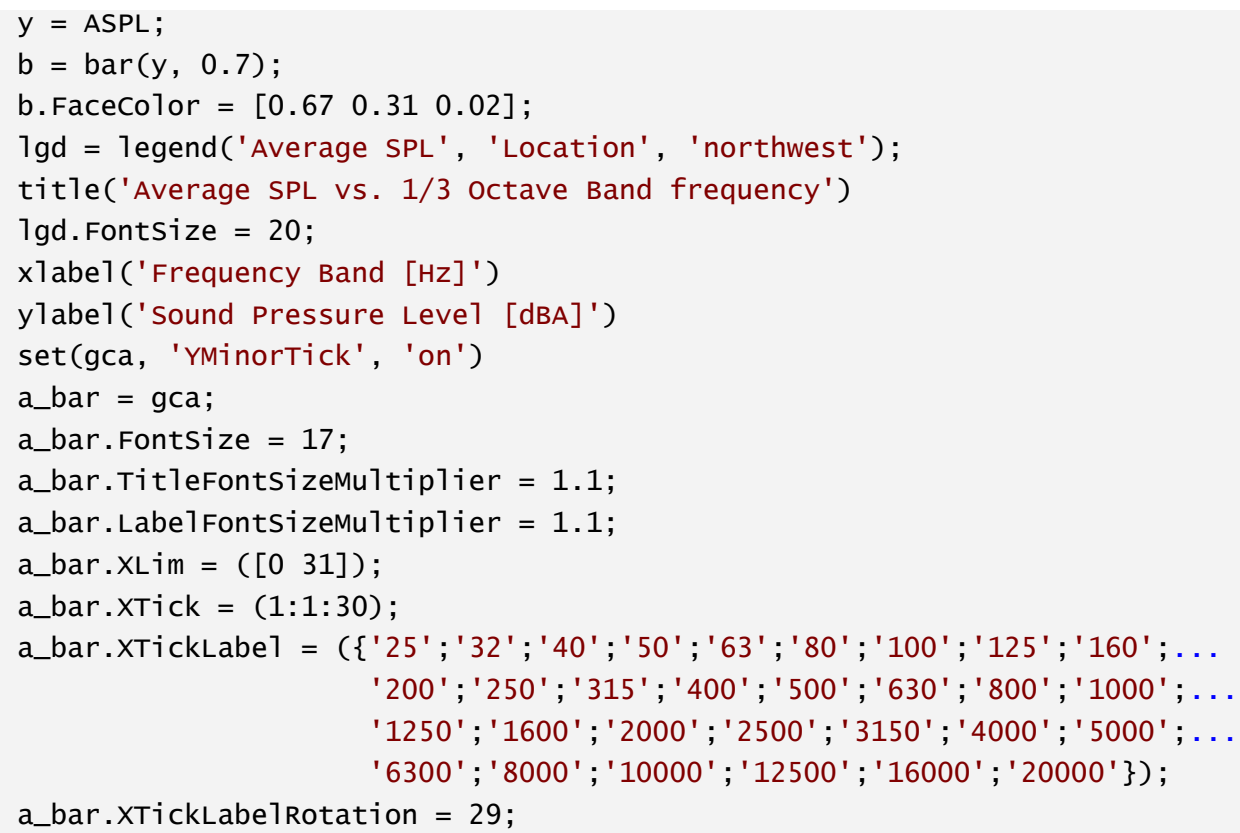




\section{Appendix D}

\section{Transient Sound Pressure and CWT Plots}

Sample plots of the recorded sound pressure of latch. The recordings are from MP1 Trial 1 at $0.851 \mathrm{~m} / \mathrm{s}$ and $1.179 \mathrm{~m} / \mathrm{s}$.

\section{Latch 1: 0.851m/s MP1 Trial 1}

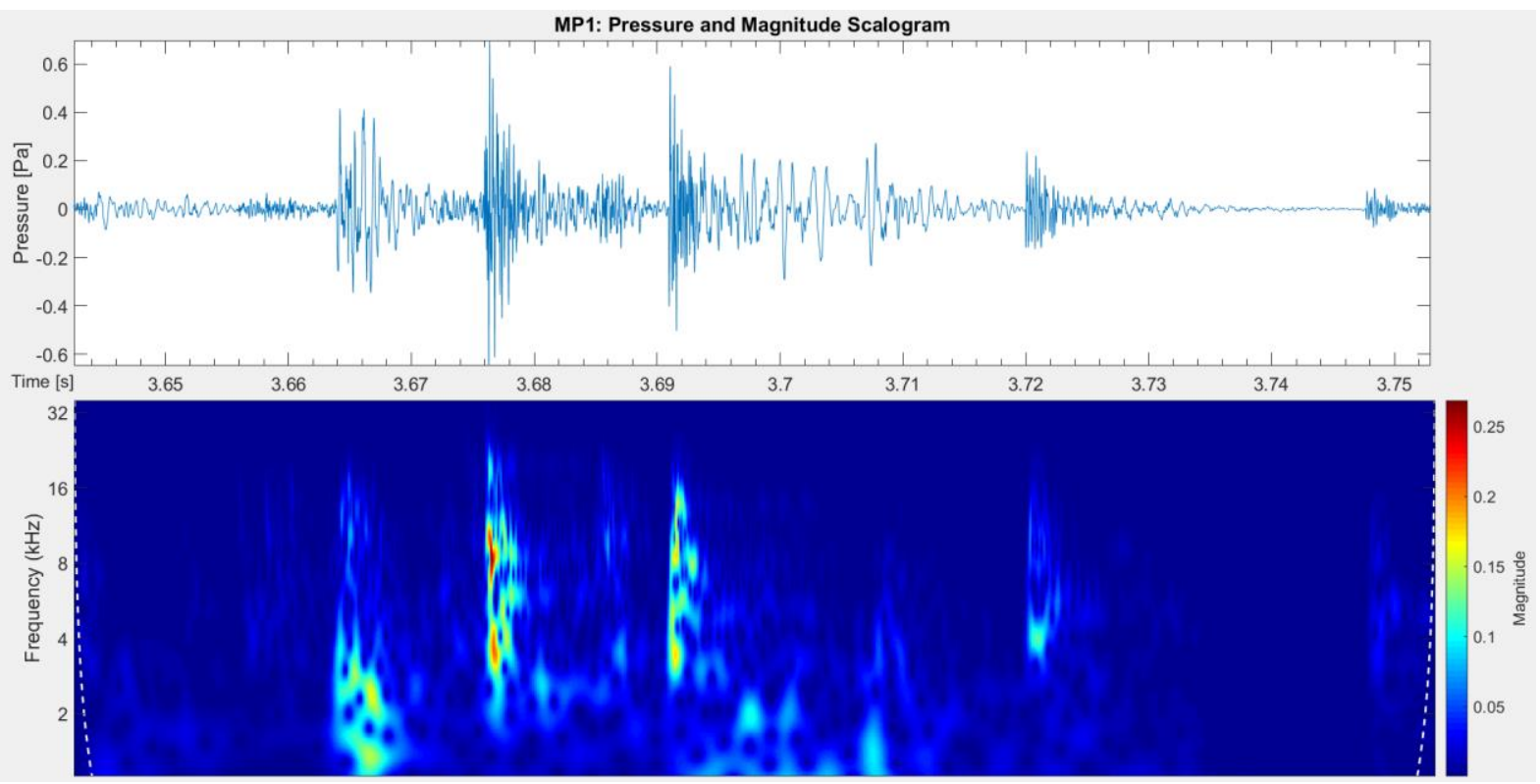

Latch 1: 1.179m/s MP1 Trial 1

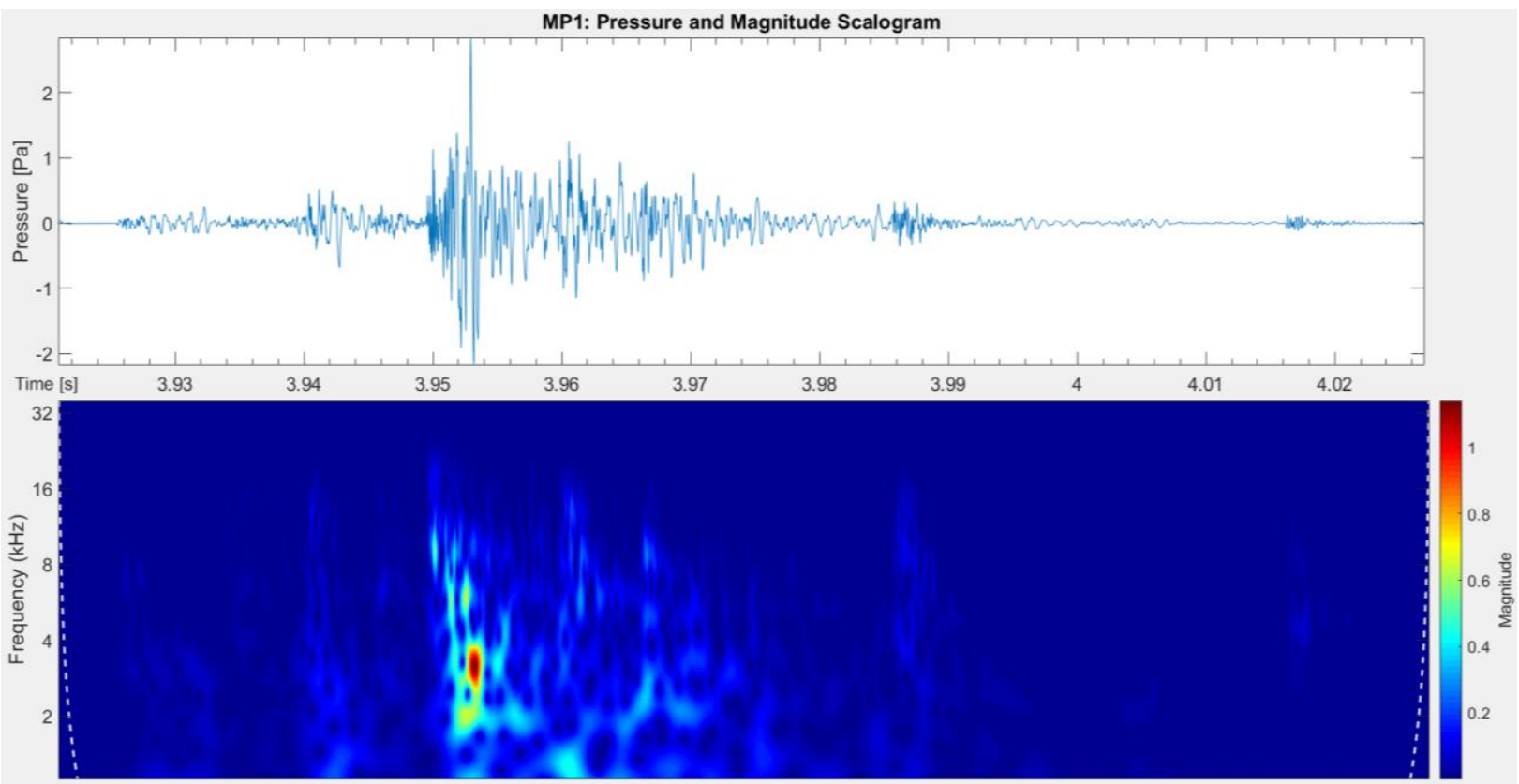




\section{Latch 2: 0.851m/s MP1 Trial 1}

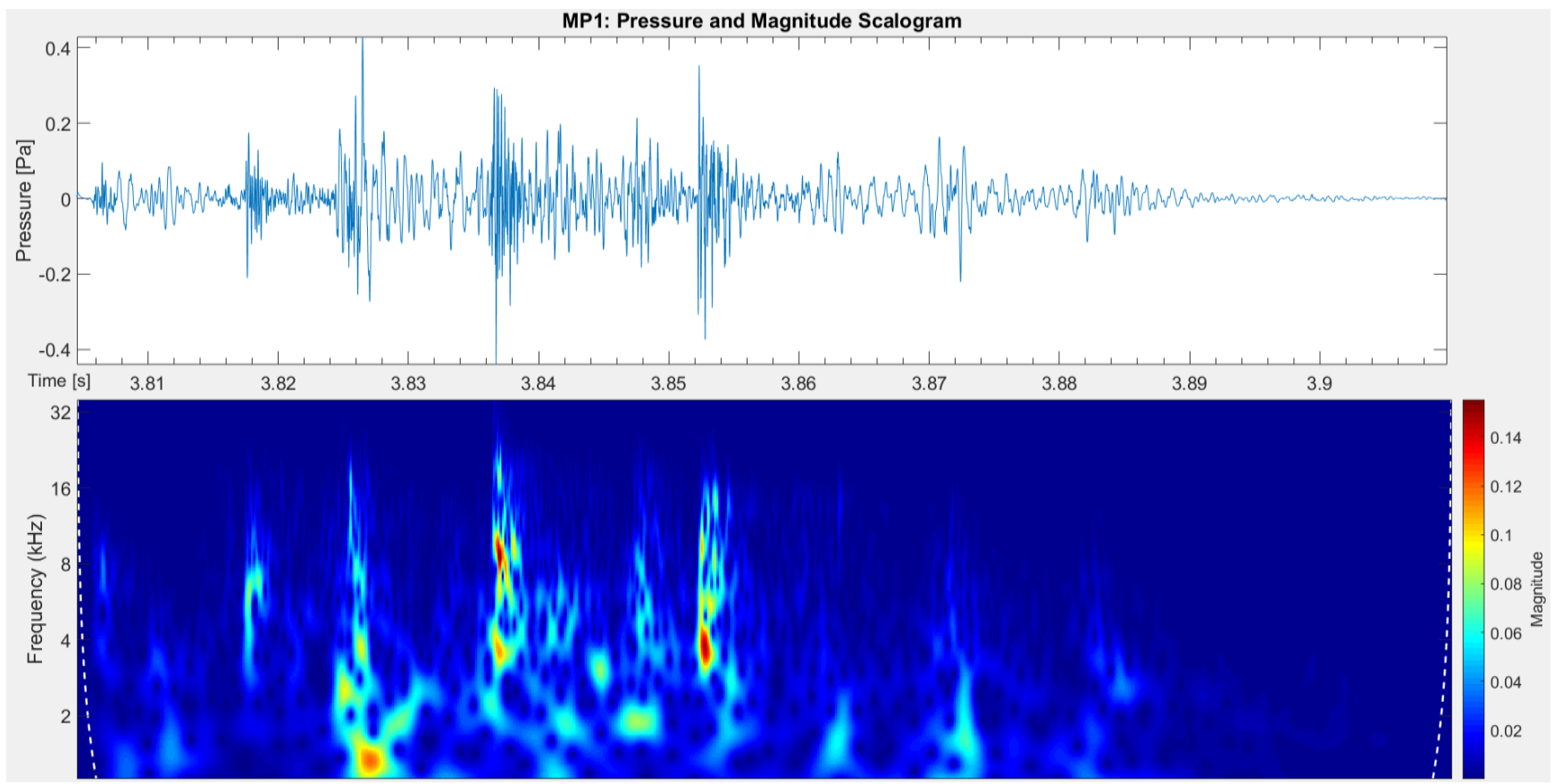

Latch 2: 1.179m/s MP1 Trial 1

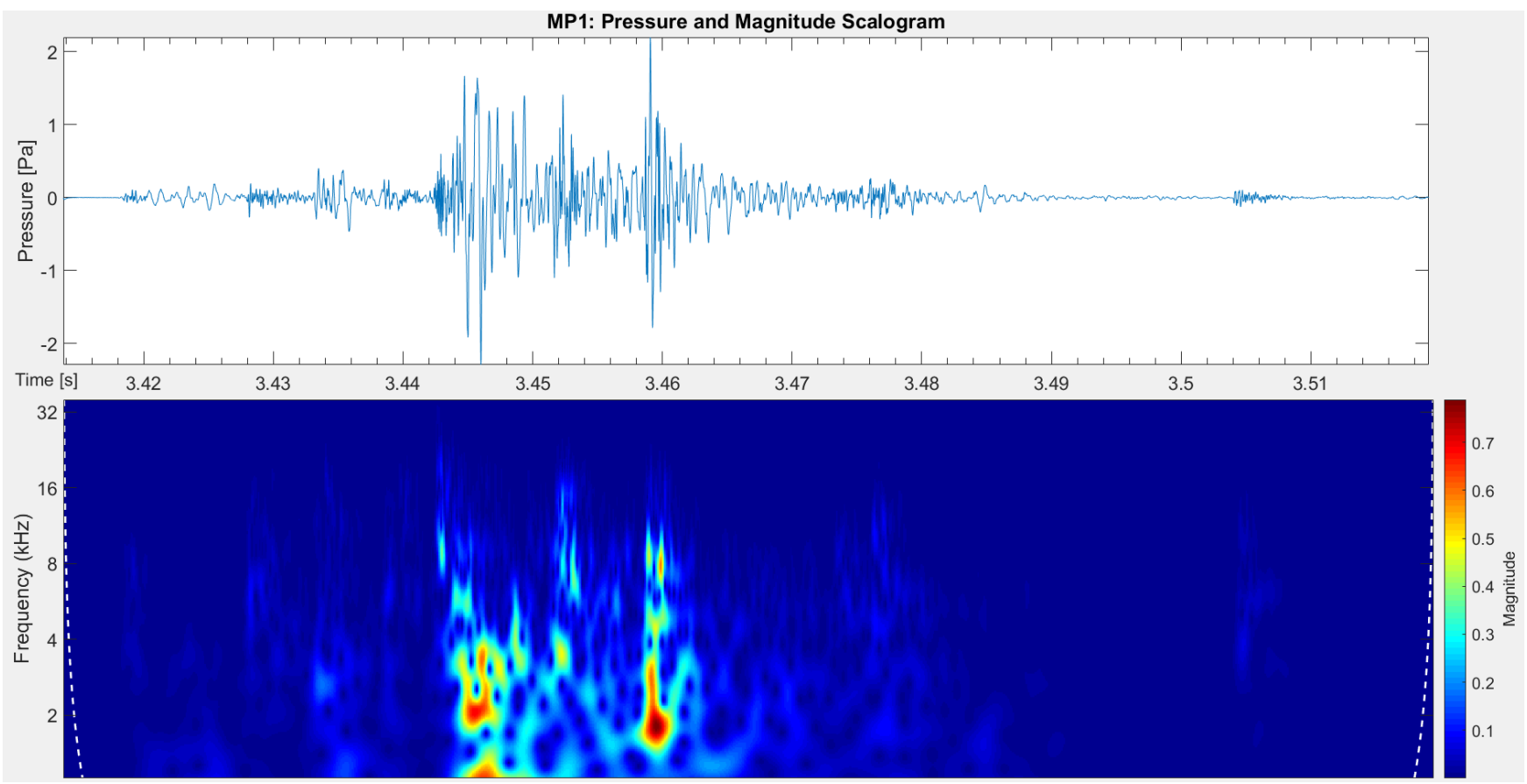




\section{Latch 3: $0.851 \mathrm{~m} / \mathrm{s}$ MP1 Trial 1}
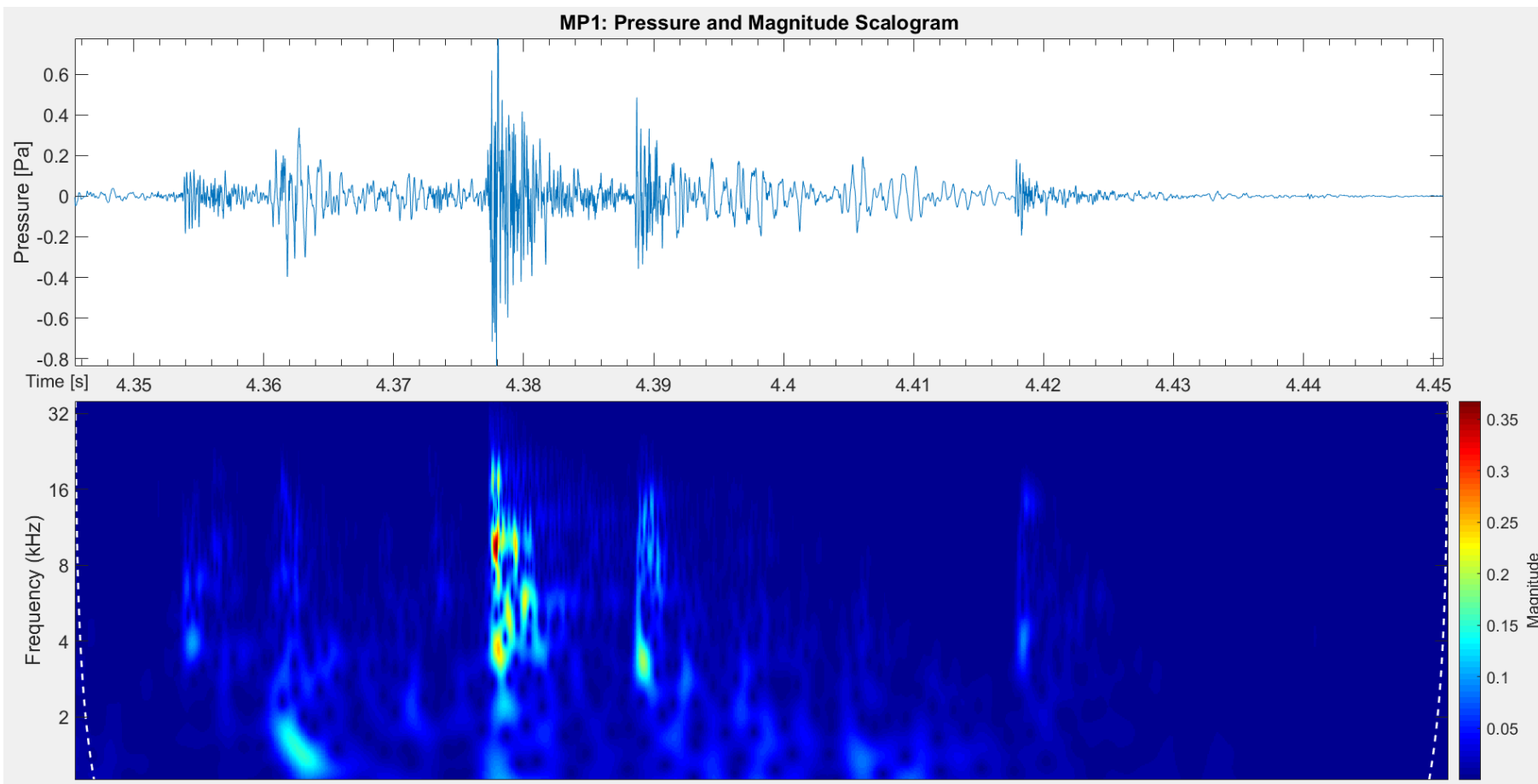

Latch 3: 1.179m/s MP1 Trial 1
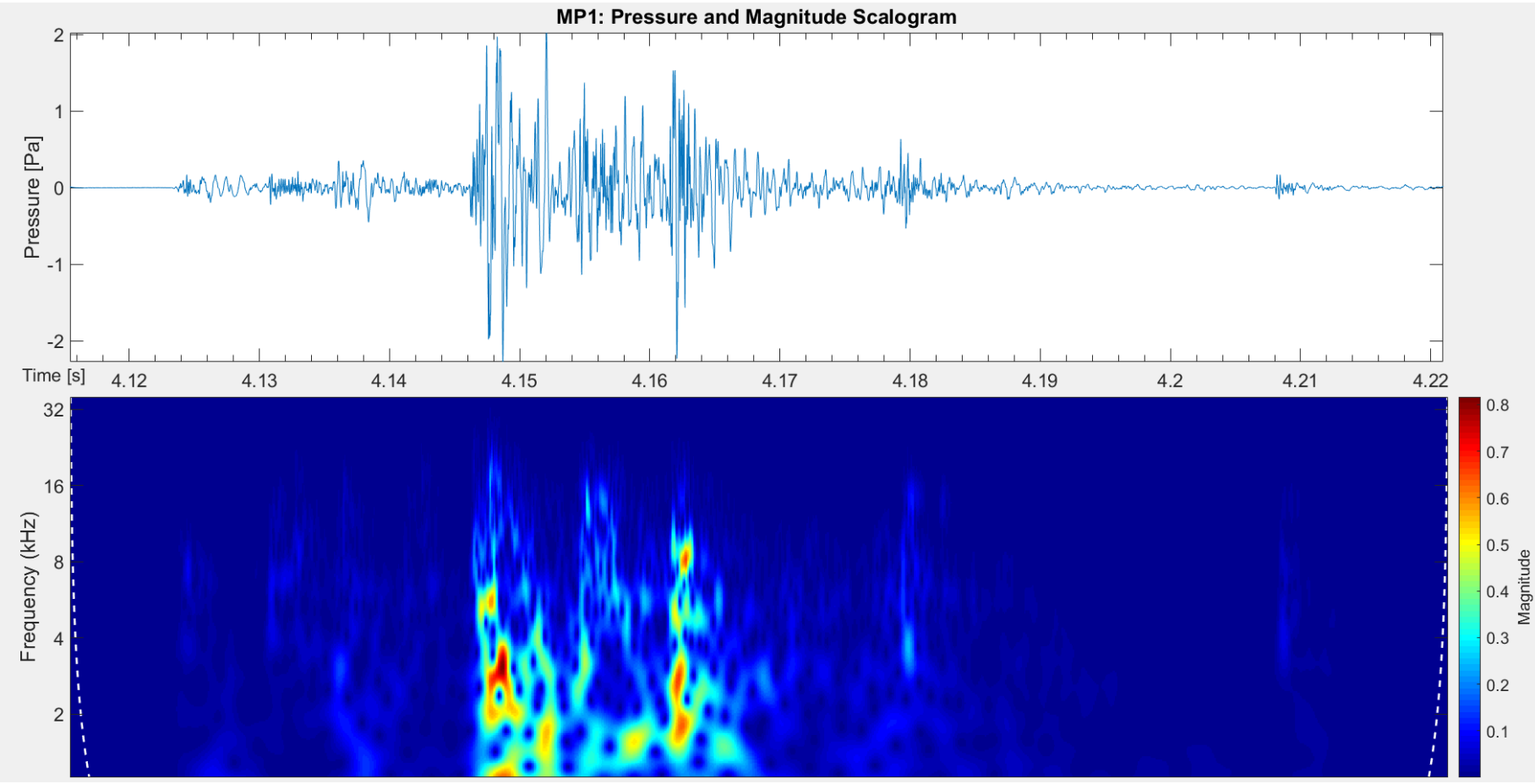


\section{Latch 4: $0.851 \mathrm{~m} / \mathrm{s}$ MP1 Trial 1}

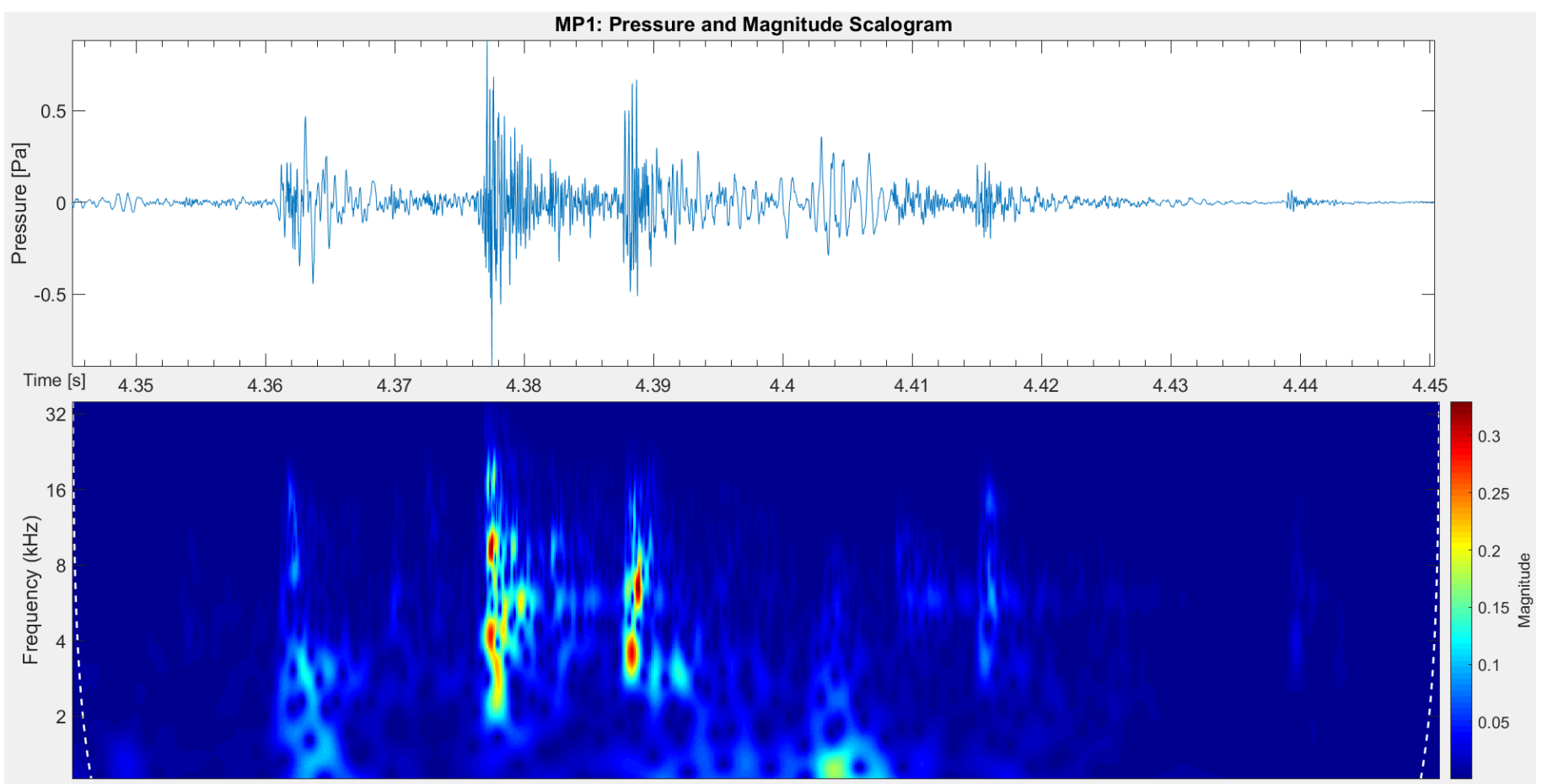

Latch 4: 1.179m/s MP1 Trial 1

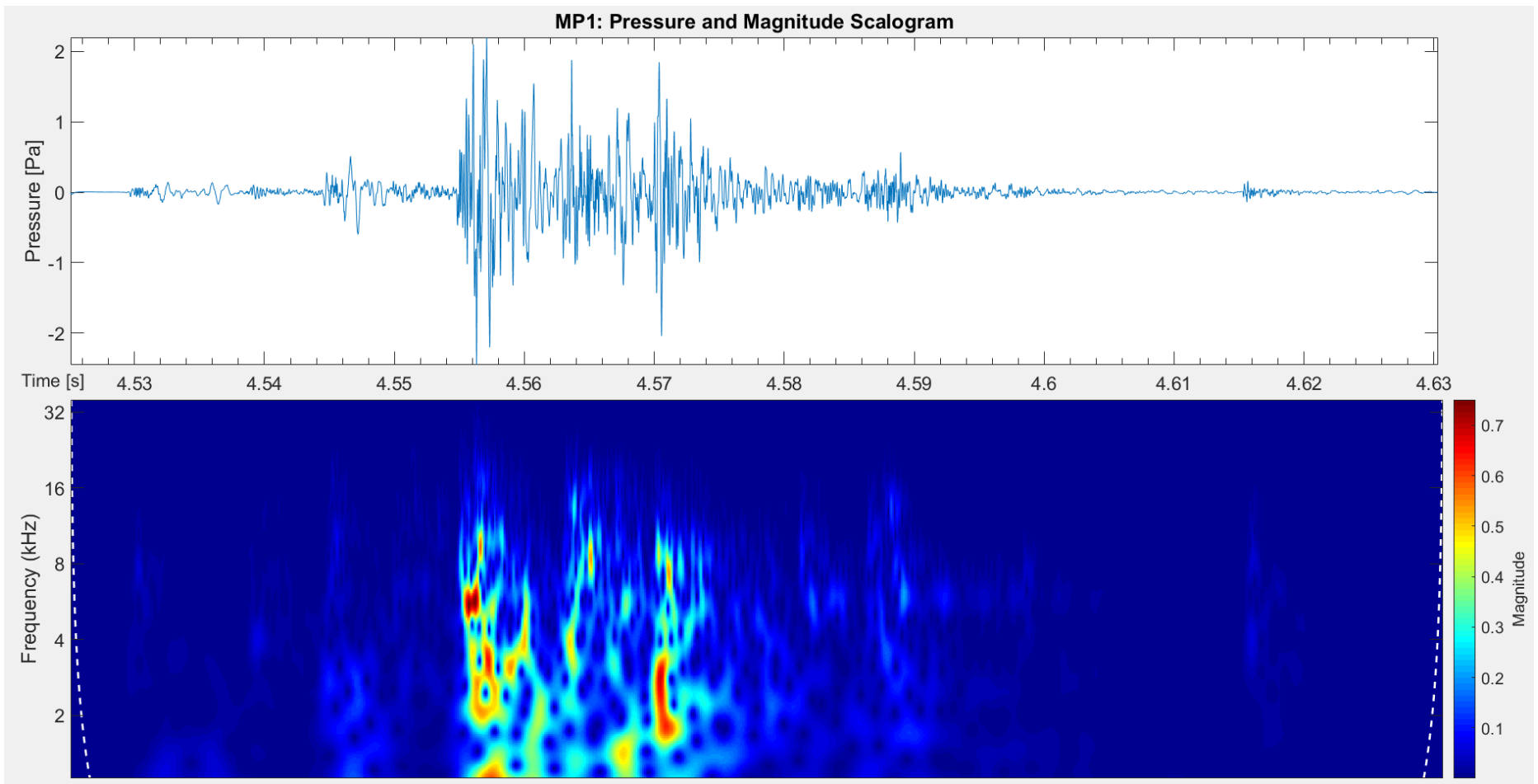




\section{Latch 5: $0.851 \mathrm{~m} / \mathrm{s}$ MP1 Trial 1}

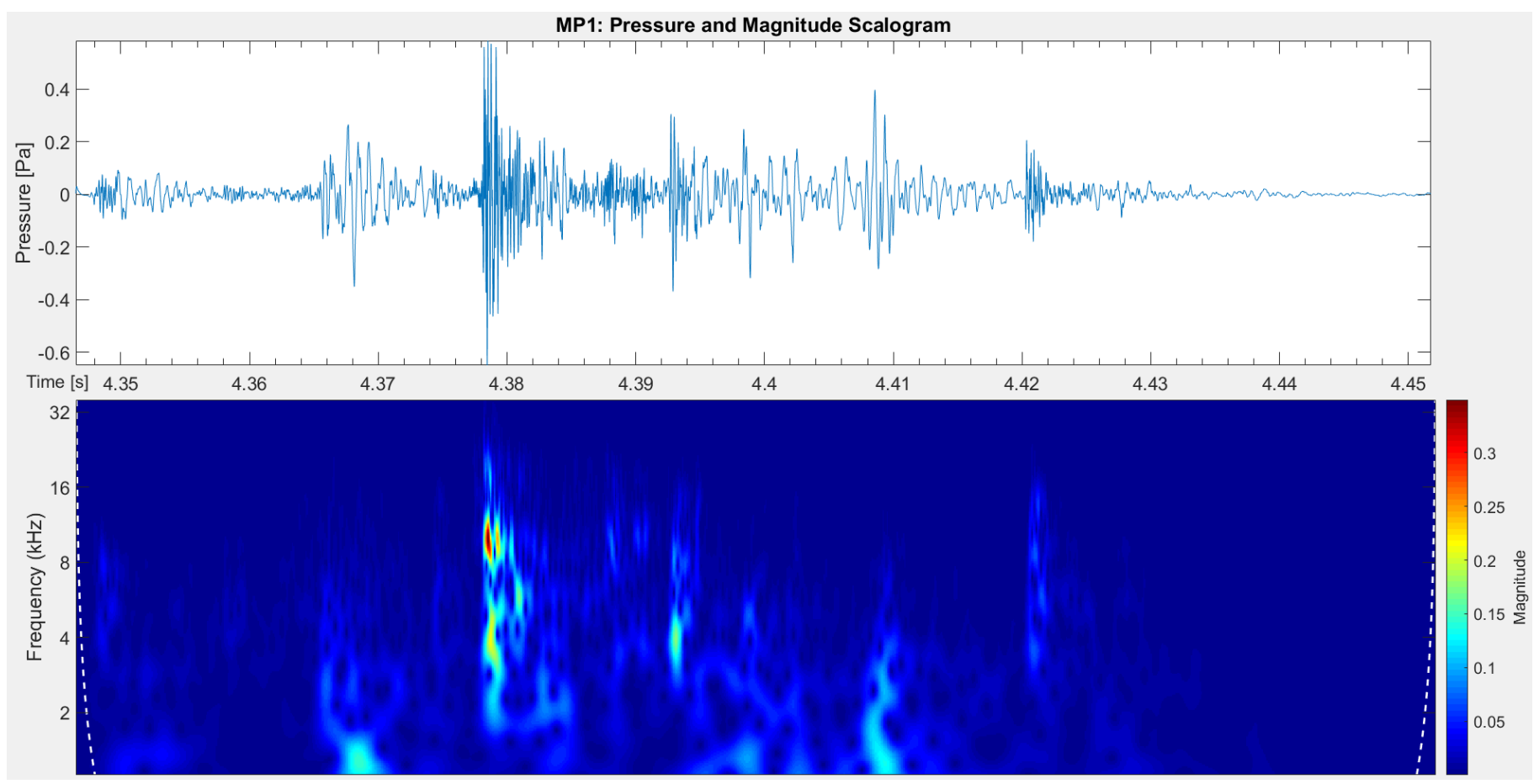

Latch 5: 1.179m/s MP1 Trial 1

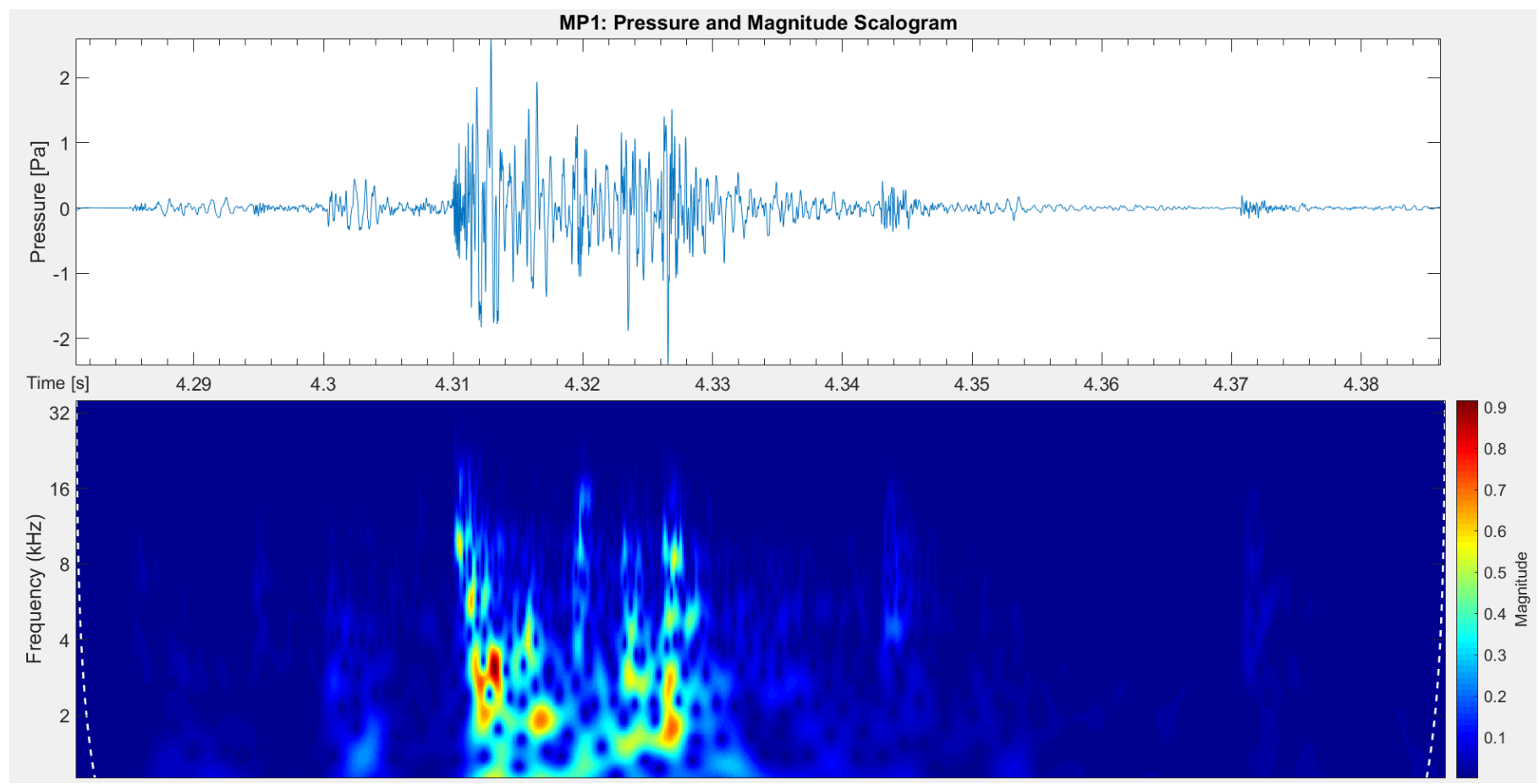




\section{Appendix E}

\section{Tabular Average Sound Pressure Level}

\section{Latch 1}

\begin{tabular}{|c|c|c|c|}
\hline $\mathbf{1 / 3}$ Octave Band & SPL at $\mathbf{0 . 8 5 1} \mathbf{m}$ [ [dBA] & SPL at 1.179m/s [dBA] & Percent Difference [\%] \\
\hline $\mathbf{1 6 0}$ & 21.59 & 35.58 & 64.78 \\
\hline $\mathbf{2 0 0}$ & 30.83 & 38.49 & 24.82 \\
\hline $\mathbf{2 5 0}$ & 35.90 & 45.62 & 27.07 \\
\hline $\mathbf{3 1 5}$ & 37.86 & 47.25 & 24.81 \\
\hline $\mathbf{4 0 0}$ & 37.79 & 47.70 & 26.21 \\
\hline $\mathbf{5 0 0}$ & 45.49 & 55.83 & 22.74 \\
\hline $\mathbf{6 3 0}$ & 52.40 & 63.33 & 20.86 \\
\hline $\mathbf{8 0 0}$ & 54.13 & 68.49 & 26.52 \\
\hline $\mathbf{1 0 0 0}$ & 56.18 & 68.82 & 22.50 \\
\hline $\mathbf{1 2 5 0}$ & 58.25 & 70.03 & 20.22 \\
\hline $\mathbf{1 6 0 0}$ & 58.52 & 70.38 & 20.26 \\
\hline $\mathbf{2 0 0 0}$ & 62.11 & 71.12 & 14.51 \\
\hline $\mathbf{2 5 0 0}$ & 61.04 & 72.79 & 19.25 \\
\hline $\mathbf{3 1 5 0}$ & 58.17 & 73.21 & 25.86 \\
\hline $\mathbf{4 0 0 0}$ & 58.31 & 70.04 & 20.11 \\
\hline $\mathbf{5 0 0 0}$ & 57.52 & 68.58 & 19.23 \\
\hline
\end{tabular}

\section{Latch 2}

\begin{tabular}{|c|c|c|c|}
\hline $\mathbf{1 / 3}$ Octave Band & SPL at $\mathbf{0 . 8 5 1} \mathbf{m} \mathbf{s}[\mathbf{d B A}]$ & SPL at $\mathbf{1 . 1 7 9 m} / \mathbf{s}[\mathbf{d B A}]$ & Percent Difference [\%] \\
\hline $\mathbf{1 6 0}$ & 22.89 & 35.92 & 56.94 \\
\hline $\mathbf{2 0 0}$ & 31.05 & 38.25 & 23.18 \\
\hline $\mathbf{2 5 0}$ & 35.62 & 45.56 & 27.89 \\
\hline $\mathbf{3 1 5}$ & 38.60 & 47.49 & 23.03 \\
\hline $\mathbf{4 0 0}$ & 37.59 & 48.07 & 27.89 \\
\hline $\mathbf{5 0 0}$ & 44.69 & 55.59 & 24.38 \\
\hline $\mathbf{6 3 0}$ & 48.73 & 62.93 & 29.16 \\
\hline $\mathbf{8 0 0}$ & 51.70 & 68.29 & 32.08 \\
\hline $\mathbf{1 0 0 0}$ & 55.31 & 69.07 & 24.88 \\
\hline $\mathbf{1 2 5 0}$ & 55.56 & 69.94 & 25.88 \\
\hline $\mathbf{1 6 0 0}$ & 57.51 & 70.55 & 22.68 \\
\hline $\mathbf{2 0 0 0}$ & 60.14 & 71.84 & 19.45 \\
\hline $\mathbf{2 5 0 0}$ & 59.22 & 71.26 & 20.33 \\
\hline $\mathbf{3 1 5 0}$ & 58.10 & 72.57 & 24.91 \\
\hline $\mathbf{4 0 0 0}$ & 57.53 & 69.12 & 20.16 \\
\hline $\mathbf{5 0 0 0}$ & 54.25 & 68.67 & 26.60 \\
\hline
\end{tabular}




\section{Latch 3}

\begin{tabular}{|c|c|c|c|}
\hline $\mathbf{1 / 3}$ Octave Band & SPL at $\mathbf{0 . 8 5 1} \mathbf{m}$ [ [dBA] & SPL at $\mathbf{1 . 1 7 9 m} / \mathbf{s}$ [dBA] & Percent Difference [\%] \\
\hline $\mathbf{1 6 0}$ & 24.53 & 35.49 & 44.67 \\
\hline $\mathbf{2 0 0}$ & 33.22 & 38.98 & 17.32 \\
\hline $\mathbf{2 5 0}$ & 35.13 & 46.50 & 32.36 \\
\hline $\mathbf{3 1 5}$ & 40.66 & 47.99 & 18.04 \\
\hline $\mathbf{4 0 0}$ & 38.51 & 48.01 & 24.65 \\
\hline $\mathbf{5 0 0}$ & 46.11 & 55.90 & 21.24 \\
\hline $\mathbf{6 3 0}$ & 51.51 & 62.88 & 22.06 \\
\hline $\mathbf{8 0 0}$ & 53.81 & 67.90 & 26.18 \\
\hline $\mathbf{1 0 0 0}$ & 57.17 & 69.54 & 21.63 \\
\hline $\mathbf{1 2 5 0}$ & 57.13 & 70.65 & 23.66 \\
\hline $\mathbf{1 6 0 0}$ & 60.64 & 71.05 & 17.17 \\
\hline $\mathbf{2 0 0 0}$ & 60.06 & 71.22 & 18.58 \\
\hline $\mathbf{2 5 0 0}$ & 59.57 & 72.01 & 20.88 \\
\hline $\mathbf{3 1 5 0}$ & 60.20 & 71.95 & 19.52 \\
\hline $\mathbf{4 0 0 0}$ & 58.87 & 69.39 & 17.87 \\
\hline $\mathbf{5 0 0 0}$ & 56.49 & 69.52 & 23.07 \\
\hline
\end{tabular}

\section{Latch 4}

\begin{tabular}{|c|c|c|c|}
\hline $\mathbf{1 / 3}$ Octave Band & SPL at 0.851 m/s [dBA] & SPL at 1.179m/s [dBA] & Percent Difference [\%] \\
\hline $\mathbf{1 6 0}$ & 23.81 & 36.31 & 52.50 \\
\hline $\mathbf{2 0 0}$ & 32.97 & 38.32 & 16.25 \\
\hline $\mathbf{2 5 0}$ & 36.83 & 46.89 & 27.31 \\
\hline $\mathbf{3 1 5}$ & 40.69 & 48.49 & 19.17 \\
\hline $\mathbf{4 0 0}$ & 38.93 & 48.72 & 25.15 \\
\hline $\mathbf{5 0 0}$ & 47.79 & 55.45 & 16.02 \\
\hline $\mathbf{6 3 0}$ & 51.94 & 63.04 & 21.37 \\
\hline $\mathbf{8 0 0}$ & 53.68 & 68.29 & 27.21 \\
\hline $\mathbf{1 0 0 0}$ & 57.67 & 69.10 & 19.82 \\
\hline $\mathbf{1 2 5 0}$ & 57.61 & 69.92 & 21.38 \\
\hline $\mathbf{1 6 0 0}$ & 57.05 & 70.44 & 23.48 \\
\hline $\mathbf{2 0 0 0}$ & 57.69 & 70.74 & 22.62 \\
\hline $\mathbf{2 5 0 0}$ & 60.51 & 71.40 & 17.99 \\
\hline $\mathbf{3 1 5 0}$ & 61.84 & 72.96 & 17.99 \\
\hline $\mathbf{4 0 0 0}$ & 60.32 & 70.58 & 17.01 \\
\hline $\mathbf{5 0 0 0}$ & 58.59 & 69.90 & 19.30 \\
\hline
\end{tabular}




\section{Latch 5}

\begin{tabular}{|c|c|c|c|}
\hline 1/3 Octave Band & SPL at $0.851 \mathrm{~m} / \mathrm{s}[\mathrm{dBA}]$ & SPL at $1.179 \mathrm{~m} / \mathrm{s}[\mathrm{dBA}]$ & Percent Difference [\%] \\
\hline 160 & 22.47 & 36.56 & 62.70 \\
\hline 200 & 31.70 & 38.93 & 22.79 \\
\hline 250 & 37.39 & 45.77 & 22.42 \\
\hline 315 & 39.62 & 47.11 & 18.91 \\
\hline 400 & 36.89 & 48.64 & 31.85 \\
\hline 500 & 45.19 & 55.37 & 22.53 \\
\hline 630 & 50.77 & 63.65 & 25.38 \\
\hline 800 & 52.29 & 68.74 & 31.46 \\
\hline 1000 & 56.72 & 70.25 & 23.86 \\
\hline 1250 & 56.64 & 70.17 & 23.89 \\
\hline 1600 & 57.22 & 72.63 & 26.93 \\
\hline 2000 & 60.17 & 71.75 & 19.25 \\
\hline 2500 & 62.86 & 73.06 & 16.23 \\
\hline 3150 & 58.94 & 73.32 & 24.39 \\
\hline 4000 & 58.78 & 70.57 & 20.05 \\
\hline 5000 & 57.00 & 69.88 & 22.58 \\
\hline
\end{tabular}




\section{Appendix F \\ Rigid Body Dynamics: Coil Spring to Torsional Spring Conversion}

A conversion of the coil spring values to equivalent torsional values was required to model the kinematics of the pawl, ratchet and striker during operation. The relationships are expressed in Equation F.1 and F.2.

$$
\begin{aligned}
& \kappa=\left(k_{L}\right)(r)^{2} \\
& T_{I}=\left(F_{I}\right)(r)
\end{aligned}
$$

where $\kappa$ is the equivalent torsional spring constant, $k_{L}$ is the coil spring constant, $r$ is the length of the moment arm (radius), $T_{I}$ is the installed spring torque and $F_{I}$ is the installed force. The coil spring constant for the pawl and ratchet springs were $537.9 \mathrm{~N} / \mathrm{m}$ and $328 \mathrm{~N} / \mathrm{m}$ respectively. The installed force of the pawl spring and ratchet spring were $7 N$ and $4.2 N$ respectively. The equations and values presented were provided by Magna Closures Inc.

It was difficult to obtain precise measurements for the pawl and ratchet coil spring compression with the real-life latch model as the springs were placed in a curved recess within the latch housing. Instead, the compression measurements were calculated using the D21MC CAD model in ANSYS SpaceClaim. Virtual construction lines allowed for more accurate tracking of the spring compression during operation. As a simplification, the curved motion of each spring was assumed to follow the path of a circle with a fixed radius. This can be seen in Figure F.1. 


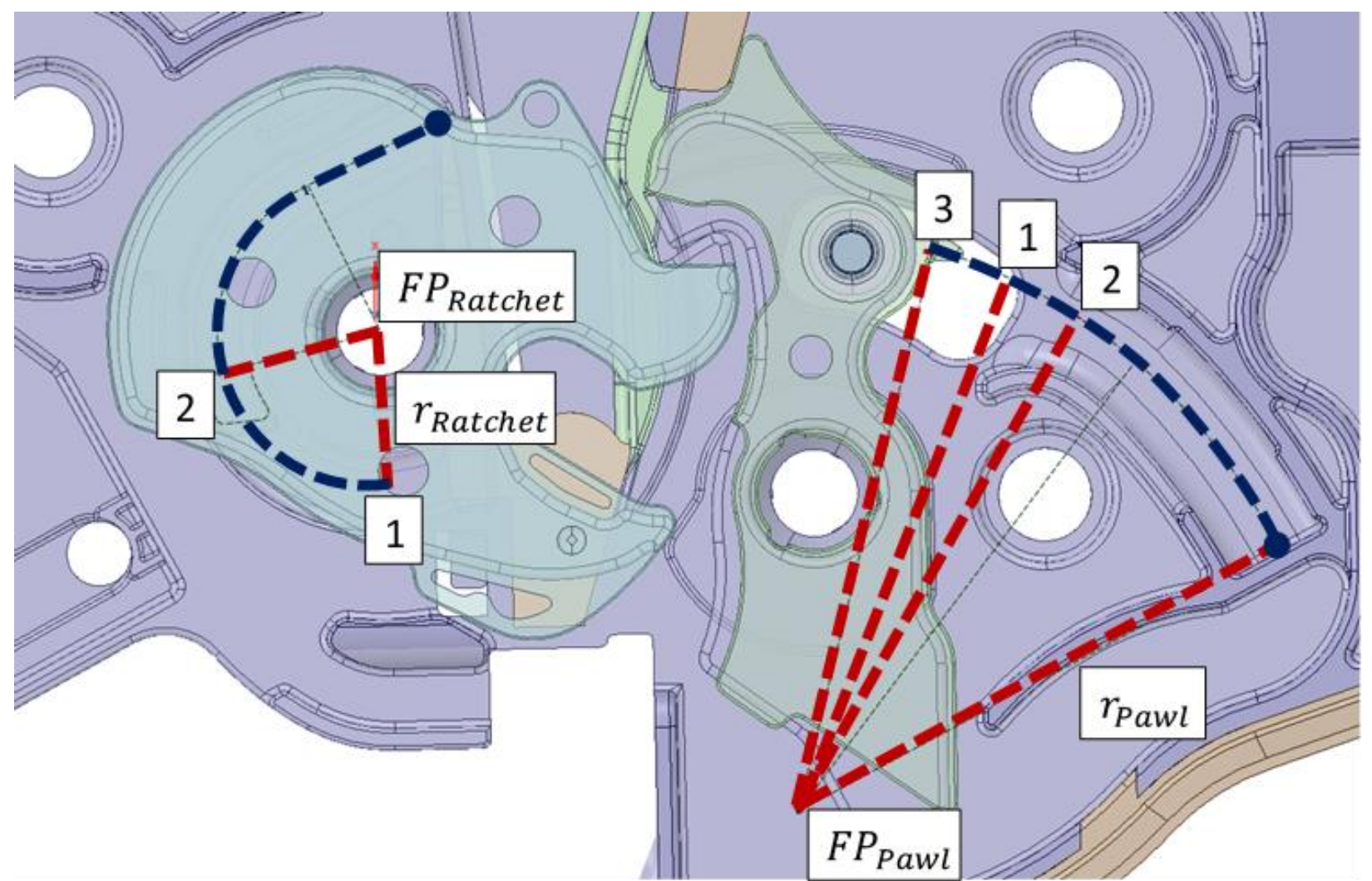

Figure F.1: Graphic of the ratchet and pawl spring compression during operation. 1) Installed

length, 2) Max compression length, 3) Fully latched position. The solid blue circle indicates the fixed base of the spring.

In Figure F.1, the motion of the pawl spring is divided into four stages denoted as installed length (IL), max compression (MC) during operation, fully latched position (FLP) and free length (FL) (not depicted). It was assumed the coil spring travelled a curved distance resembling that of a circle with a constant radius. The center, or focal point, is represented by $F P_{P a w l}$. From this the radius was measured to be $0.03915 \mathrm{~m}$. The process to determine the $\kappa$ and $F_{I}$ is shown below:

$$
\begin{aligned}
\kappa & =k_{L}(r)^{2} \\
& =537.9 \frac{\mathrm{N}}{\mathrm{m}}(0.03915 \mathrm{~m})^{2} \\
& =0.8245 \frac{\mathrm{Nm}}{\mathrm{rad}}
\end{aligned}
$$




$$
\begin{aligned}
T_{I} & =F_{I}(r) \\
& =7 N(0.03915 m) \\
& =0.27 \mathrm{Nm}
\end{aligned}
$$

A similar method was performed to determine the equivalent torsional spring constant and installed torque of the ratchet spring. The motion of the ratchet spring was divided into three stages denoted as installed length (IL), max compression during operation (MC) and free length (FL) (not depicted). This is viewed in Figure F.1. In this case, the MC of the ratchet spring also represents the length at the FLP. Similarly, it was assumed the coil spring travelled a curved distance resembling that of a circle with a constant radius. The focal point is represented by $F P_{\text {Ratchet }}$. From this the radius was measured to be $0.0114 \mathrm{~m}$. The process to determine the $\kappa$ and $F_{I}$ is shown below:

$$
\begin{aligned}
\kappa & =k_{L}(r)^{2} \\
& =328 \frac{N}{m}(0.0114 m)^{2} \\
& =0.0426 \frac{\mathrm{Nm}}{\mathrm{rad}} \\
T_{I} & =F_{I}(r) \\
& =4.2 \mathrm{~N}(0.0114 \mathrm{~m}) \\
& =0.0479 \mathrm{Nm}
\end{aligned}
$$

The torsional spring constant and installed torque were calculated using a second method (not included in this work) which provided very similar results. Therefore, the results were concluded to be accurate and it was deemed appropriate to move forward with the computational analysis. 


\section{Appendix G}

\section{Computational Analysis Flow-Chart}

\section{Input Conditions}

- Striker entrance speeds used during experimental analysis

- $0.851 \mathrm{~m} / \mathrm{s}$ and $1.179 \mathrm{~m} / \mathrm{s}$

\section{Rigid Body Dynamics (Kinematic)}

- Tangential impact speed of the latch components

- Pawl/Ratchet

- Striker/Over-Slam Bumper \#1

- Ratchet/Housing

- Pawl/Over-Slam Bumper \#2

- Assumptions and Simplifications: All bodies were rigid/frictionless; coil springs modelled as equivalent torsional springs; measured speeds were approximations of the real life kinematics

- Output: Tangential impact speed of the latch components

\section{Explicit Dynamics (Dynamic)}

- Reaction forces of the latch components

- Pawl/Ratchet

- Striker/Over-Slam Bumper \#1

- Ratchet/Housing

- Pawl/Over-Slam Bumper \#2

- Assumptions and Simplifications: Each impact was modelled separately; all components modelled as isotropic, linearly elastic materials; simulation type was Deformable/Deformable

- Output: Striker/Over-Slam Bumper \#1 impact force

\section{Transient Structural (Acoustic)}

- Only the Striker/Over-Slam Bumper \#1 impact was modelled

- 6 acoustic simulations performed in total

- Frequency Bandwidth: $160 \mathrm{~Hz}$ to $5 \mathrm{kHz}$

- Assumptions and Simplifications: 'Slivered' acoustic region with a curved outer face; all SOLID185 element variations used to discretize the model; reaction forces were used as opposed to component surface velocities

- Output: Simulated sound pressure at various microphone locations

- MP1, MP1_Mid, MP1_Close, MP2, MP2_Mid, MP2_Close, MP3, MP3_Mid, MP3_Close 